CRYSTAL STRUCTURES OF THROMBIN AS SIGNPOSTS TO ITS MULTIPLE FUNCTIONS

W. Bode, M. Bauer, H. Brandstetter, and M. Stubbs

Thrombin is a multifunctional protein; it possesses both proteolytical and hormone-like properties that can be both pro- and anticoagulant. We have solved and refined the $x$-ray crystal structure of the D-Phe-Pro-Arg- $\mathrm{CH}_{2}$-human $\alpha$-thrombin, analyzed its characteristic polypeptide fold, sufface structure and electrostatic properties, and proposed a chymotrypsinogen numbering for thombin. This PRACK-thrombin model has spawned a number of other structures of human and bovine thrombin with hirudin, with fibrinopeptide $A$ and with srall peptidic and nonpeptidic inhibitors. Most of the characteristic interaction and specificity properties of thrombin can be attributed to its prominent canyon-like active-site cleft which is mainly shaped by two large insertion loops. The charged residues of thrombin are clustered to form a sandwich-like electrostatic potential, with two extended positively charged surface patches which presumably represent the heparin binding site and the fibrinogen secondary binding exosite of thrombin. Fibrinopeptide $A$ associates with thrombin in a compact manner slotting with hydrophobic residues into the apolar binding site. Hirudin binds differently from "canonical' protein inhibitors, with its negatively charged carboxyl tail wrapping around thrombin along the putative fibrinogen secondary binding site. The different interaction sites of thrombin and its mode of interaction with ligands, substrates and inhibitors will be presented and discussed.
APPEICATION OE PROTEIN ENGINEERING TO THE STUDY OF THE INTERACTION OF THROMBIN WITH HIRUDIN

\section{St. R. Stone}

Hirudin is a 65 residue protein that was originally isolated from the medicinal leech Hirudo medicinalis. It reacts with thrombin to form a tight complex and consequently inhibits all of the activities of thrombin including the cleavage of fibrinogen and the activation of platelets. Hirudin is absolutely specific for thrombin. In order to obtain sufficient quantities of hirudin for biochemical studies and for use as an antithrombotic, recombinant systems for the expression of hirudin have been established. Recombinant expression of hirudin also allows the specific modiftcation of particular residues by site-directed mutagenesis. By using this technique, it has been posible to evaluate the contributions made by particulax hirudin residues to the formation of the tight complex with thrombin. Hirudin reacts very rapidly with thrombin and protein engineering studies have shown that this rapid interaction is achieved by a process of "electrostatic steering". The c-terminal region of hirudin is rich in negatively charged amino acids and this region of hirudin binds to a postively-charged surface groove an thrombin. The complementary electrostatics fields produced by these two regions ensure that the two molecules are correctly oriented for the formation of the inhibited complex. While electrostatics forces are important in the formation of an initial complex, hydrophobic interactions are primarily responsible for the tight complex formation. A number of hydrophobic interaction occur with the c-terminal region of hirudin, but the most important interactions are between the $\mathrm{N}$-terminal region of hirudin and the active-site cleft of thrombin. Within the active-site cleft, hirudin uses apolar bnding sites and does not occupy the primaryspecificity pocket to which basic amino acids of substrates are bound. This novel interaction within the active-site cleft is the basis for hirudin's absolute specificity for thrombin.

4

THE USE OF X-RAY CRYSTAL STUDIES IN THE DEVELOPMENT OF BENZAMIDINE-DERTVED THROMBIN INHIBITORS

\section{J. Stürzebecher}

N $\alpha$ - (2-naphthylsulfonylglycyl) 4-amidinophenyla lanine piperidide (NAPAP) is a potent thrombin inhibitor. Even though NAPAP possesses remarkable anticoagulant activity, the compound cannot be further developed for therapeutic use: NAPAP is not orally absorbed, and it is rapidly eliminated from the circulation. However, variations and substitutions always led to a drastic loss in inhibitory activity. For designing new inhibitors with improved properties we (1) studied the pharmacokinetic behaviour of NAPAP derivatives and (2) used X-ray studies in the search of new basic structures.

From their pharmacokinetic studies it was found that the rapid elimination of NAPAP from the circulation is prolonged upon an increase of hydrophobicity. Furthermore, compounds containing a carboxyl group are absorbed to a certain extent after oral application. From the $\mathrm{x}$-ray structure of the NAPAP-thrombin complex it was deduced that NAPAP is bound so ideally to thrombin that there is virtualiy no further space for substituents. Therefore, 3-amidinophenylalanine piperidide appeared to be a promising lead structure for new inhibitors because the corresponding inhibitor complex indicated more space available for substituents.

Several novel derivatives of 3-amidinophenylalanine were synthesized. The amidino moiety and both the $\mathrm{N} \alpha-$ and the C-terminal substituent were widely varied. Some of the newly synthesized compounds are otent inhibitors of thrombin and exert improved pharmacokinetic properties. The structure-activity relationships are in agreement with the predictions of the $\mathrm{X}$-ray crystal studies.

present address: Institut $f$. Pharmakologie \& Tox. Med. Hochschule Erfurt, D-99012 Erfurt (Germany)
STRUCTURAL BASIS FOR PHENOTYPE OF DEFICIENCY OF ANTITHOMBOTIC EROTEIN C BASED ON MOLECULAR MODELLING OF MUTATIONS OF THE PROTEASE DOMAIN.

I. Griffin, I. Greengard, C. Fisher, B. Villoutreix

Familial deficiency of protein $\mathrm{C}$ is associated with inherited thrombophilia. To explore how specific missense mutations might cause observed clinical pehonotypes, known protein $C$ missense mutations were mapped onto three-dimensional homology models of the protein $C$ protease domain, and the implications for domain folding and structure were evaluated. Iype I deficiency is characterized by equivalent reductions in antigen and activity whereas Type II deficiency involves much lower functional than antigenic levels, presumably due to dysfunctional molecules in the blood. Most Type I missense mutations either replaced internal hydrophobic residues (I201T, L223F, A259V, A267T, A346D, A346V, G376D) or nearby interacting residues (I403M, T298M, $2184 \mathrm{H}$ ) thus disrupting the packing of internal hydrophobic side chains or replaced hydrophilic residues thus disxupting ion pairs (N256D, R178w). Mutations (P168L, R169w) at the activation site destablized the region containing the activation peptide structure. Most Type II mutations involved solvent-exposed residues and either were located in or near the active site region (S252N, D359N, G381S, G391S, H211Q) or were clustered in a remarkable positively charged region (R147W, R157Q, $\mathrm{R} 2290, \mathrm{R} 352 \mathrm{w})$. The cluster of arginines $147,157,229$, and 352 may identify a functionally important exosite on the surface of the protease domain of protein $c$. Identification of the spatial relationships of natural mutations in the protein $\mathrm{C}$ model is helpful for understanding manifestations of protein $C$ deficiency and for identification of novel, functionally important molecular features and exosites. 
Fibrinolysis

5

NOTOGINSENOSIDE R1 COUNTERACTS BOTH THE DOWNREGULATION OF TISSUE-TYPE PLAMINOGEN ACTIVATOR AND THE PRODUCTION OF PLASMINOGEN ACTIVATOR INHIBITOR-1 IN CULTURED HUMAN UMBILICAL VEIN ENDOTHELIAL CELLS EXPOSED TO ENDOTOXIN

W.J. ZHANG ${ }^{1,2}$, J. WOJTA ${ }^{1}$, X.L. ZHENG ${ }^{1}$, D.B. ALAZHRY ${ }^{1}$, J.C. ZHANG ${ }^{1}$ and B.R. BINDER ${ }^{1}$

We have previously reported that Notoginsenoside R1 (NRI) has an effect on the synthesis of tissue-type plasminogen activator ( $t-P A)$ in cultured human umbilical vein endothelial cells (HUVECs)(Thromb. and Haemost. 69(6):1275,1993). In this study we investigated the effect of NRI on fibrinolytic properties of cultured HUVECs exposed to endotoxin to evaluate possible modulation of endotoxin effects by NR1. The approximate $20 \%$ downregulation of $t-P A$ antigen induced by LPS (E. coli lipopolysaccharide $026: B 6,1 \mu \mathrm{g} / \mathrm{ml}$ for 12 hours) was completely prevented when the cells were coincubated with LPS and $100 \mu \mathrm{g} / \mathrm{ml}$ NRI (t-PA antigen, LPS treated cells: $2.57 \pm 0.15 \mathrm{ng} / 10^{5} \mathrm{cells}$; control cells: $3.09 \pm$ $0.17 \mathrm{ng} / 10^{5}$ cells; coincubated with both LPS and NRI cells: $3.62 \pm$ $0.13 \mathrm{ng} / 10^{5}$ cells, $\mathrm{n}=6$ ). In accordance with changes in $\mathrm{t}-\mathrm{PA}$ antigen levels in the conditioned media (CM), the $32 \%$ decrease in t-PA messenger RNA (mRNA) induced by LPS was also prevented by $100 \mu \mathrm{g} / \mathrm{ml}$ NRI. Also LPS induced PAI-1 antigen and activity in the CM of HUVECs (PAI-1 antigen, LPS treated cells: 731 $\pm 26 \mathrm{ng} / 10^{5}$ cells; control cells: $296 \pm 9.2 \mathrm{ng} / 10^{5}$ cells, $\mathrm{n}=9$, PAI-1 activity, LPS treated cells: $8.22 \pm 0.18 \mathrm{U} / 10^{5}$ cells, control cells: $5.48 \pm 0.78 \mathrm{U} / 10^{5}$ cells $n=6$ ), an effect prevented when the cells were coincubated with both LPS and $100 \mu \mathrm{g} / \mathrm{ml}$ NRI (PAI-1 antigen: $418 \pm 23 n g / 10^{5}$ cells, $n=9$, PAI-I activity $4.77 \pm$ $0.26 \mathrm{U} / 10^{5}$ cells, $\mathrm{n}=6, \mathrm{P}<0.01$ compared with LPS treated cells). The 2 -fold increase in PAI-1 mRNA levels $(3.4 \mathrm{~Kb})$ induced by LPS (1 $\mathrm{gg} / \mathrm{ml}$ for 6 hours) was only 1.37 -fold in the presence of both LPS and $100 \mu \mathrm{g} / \mathrm{ml} \mathrm{NR} 1$. The present results suggest that NRI changes t-PA and PAl-1 expression in LPS treated endothelial cells thereby effectively counteracting the antifibrinolytic potential of endothelial cells induced by endotoxin.

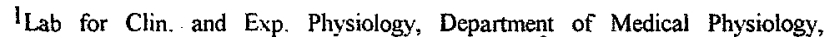
University of Vienna, A-1090 Vienna, AUSTRIA; 2 Department of Physiology, Beijing University of Traditional Chinese Medicine, Beijing 100029, P.R.CHINA

THE USE OF ENDOTHELIAL CELL PLASMA MEMBRANE VESICLES FOR THE STUDY OF FIBRINOLYTIC ENZYME KINETICS AT THE ENDOTHELLAL CELL SURFACE: H. Zoellner, R. Beckmann B.R. Binder.

Fibrin activates the fibrinolytic system, and there is some evidence that a similar activation occurs at the endothelial cell (EC) surface. In studying EC surface activation of fibrinolysis, however, it is difficult, to exclude the confounding effects of matrix proteins and proteins released from treated cells during kinetic experiments. To aid in the study of EC surface enzymatic phenomena, we are currently developing a method for the preparation of highly purified EC plasma membrane vesicles (PMV) Human EC express a molecule on the plasma membrane cell surface which binds the lectin Ulex Europaeus-1 (UEA-1). This binding has been exploited for the purification of EC from mixed cell populations, and we have adapted this approach to obtain highly purified PMV from cultured human umbilical vein EC. Briefly, EC are lysed in Tris $0.005 \mathrm{M}$, pH 8.2, EDTA 0.001M, Sucrose 0.25M, to yield a preparation of PMV mixed with nuclei, other cellular membranes and some intact whole cells. The remaining whole cells and nuclei are removed by filtration. Sehpacryl-HR 200 beads are used for filtration, by re-suspending the lysed cell preparation in an equal volume of beads, and pumping lysis buffer through the cell-bead slurry in a standard chromatography column. Whole cells and nuclei are trapped by the beads, while a mixed vesicle preparation flows through the column and can be concentrated by centrifugation. This preparation is then applied to a UEA-1Sepharose affinity column, and phosphate buffered saline with $0.001 \mathrm{M}$ EDTA used to wash non-adherent vesicles from the gel. Alpha-LFucose is used to elute UEA-1 binding vesicles, which are then washed and used in experiments. Lectin-immuno-histochemistry is used to verify the identity of PMV. The effect of PMV upon fibrinolytic activity is then studied using purified fibrinolytic proteins.

Dept. of Medical. Physiology, University of Vienna.
7

HEPATOCYTE GROWTH FACTOR (HGF) STIMULATES PLASMINOGEN ACTIVATOR INHIBITOR I (PAI-1) AND TISSUE FACTOR (TF) EXPRESSION IN HepG2 CELLS. J. Wojta, T. Nakamura, P. Hufnagl, A. Fabry, R. Beckmann, K. McGrath, B. R. Binder

HGF is a powerful mitogen for both rat and human hepatocytes, epithelial cells and endothelial cells in vitro and is angiogenic in vivo. It has considerable homology with plasminogen and has been shown to upregulate urokinase-type plasminogen activator (u-PA) in endothelial cells and u-PA and its receptor in kidney epithelial cells. In this study we report that human recombinat HGF stimulates expression of PAI-I and TF in the human hepatoma cell line HepG2. PAI-1 antigen as determined by a specific ELISA increased up to three-fold in conditioned media of HepG2. This increase was dose dependent with maximum stimulation achieved with a concentration of $50 \mathrm{ng} / \mathrm{ml}$ of HGF. PAI-1 antigen also increased up to four-fold in the extracellular matrix in HGF treated HepG2. The production of the PAI-1 binding protein vitronectin $(\mathrm{Vn})$ was not affected by HGF. In contrast TF activity in HepG2 treated with HGF increased up to two-fold. As determined by Northern blotting, PAI-l and TF specific mRNA were increased significantly in the presence of HGF whereas Vn mRNA was not affected. The increase in PAI-1 and TF mRNA was also seen when HepG2 were incubated with HGF in the presence of cycloheximide, thereby indicating that de novo protein synthesis is not required to mediate the effect. In conclusion our data gives evidence that HGF in addition to its proliferative effect for different cell types is also involved in the regulation of fibrinolysis and coagulation. One could speculate that HGF might modulate processes requiring matrix degradation by increasing the expression of the protease u$\mathrm{PA}$ in one cell type and by upregulating the expression of the serine protease inhibitor PAI- 1 in a different cell type. Since u-PA has been shown to activate latent HGF to the active form, it could furthermore be speculated that by upregulating PAI-1 which in turn could inhibit u-PA, HGF might regulate its own activation.

Depts. Med. Physiol., Univ. Vienna; Biochem., Univ. Osaka; Diag. Haem., Royal Melbourne Hospital.

\section{8}

RELATIONS BETWEEN HYPERLIPEMIA, HYPERINSULINEMIA AND FIBRINOLYSIS IN RABBITS D.B. Al-Azhary
Dept. Med. Physiol., Univ. Vienna, Austria;
${ }^{2}$, Jinder ${ }^{1}$ Dept. of Zoology, Univ. El-Minia, Egypt.

The aim of this study was to investigate the effect of the long-term intake of a hyperlipemic diet and daily injections of insulin on blood lipid categories and the fibrinolytic system. Male New Zealand white rabbits were divided into 3 groups, of 6 rabbits each. The control group received normal pellet food. The second group received a hyperlipemic diet consisting of $70 \%$ normal pellets, $14 \%$ natural fat, $14 \%$ corn oil and $2 \%$ cholesterol. The third group was injected daily with $2.5 \mathrm{IE} / \mathrm{kg}$ insulin. After 28 days all animals were sacrificed and samples of blood and aorta were obtained. Plasma and serum total lipids, triglycerides, total cholesterol, HDL-cholesterol, tissue plasminogen activator $(t-P A)$ and plasminogen activator inhibitor-1 (PAI-1) were investigated. Furthermore the effect of addition of inactivated hyperlipemic serum $(1 \%)$ and insulin $(10 \mathrm{ug} / \mathrm{ml})$ on the fibrinolytic potential of normal rabbit aortic endothelial cells (RAEC) was investigated. In the hyperlipemic group, a significant increase of blood total lipids, triglycerides, total cholesterol and HDLcholesterol correlated with a significant increase of both t-PA and PAI-1 in plasma. In the hyperinsulemic group, an increase of total lipids, triglycerides and a significant decrease of HDL-cholesterol was associated with increased PAI-activity. Addition of hyperlipemic serum to RAEC induced an increased activity of PAI-1 in conditioned media, while no significant increase was seen after addition of insulin. We conclude that hyperlipemic diets induce an increase of $t-P A$ and PAI- 1 in plasma and that hyperlipemic serum has a direct effect on endothelial cell synthesis of PAI-1. Furthermore our results give evidence that insulin does not directly affect PAI-1 synthesis in endothelial cells. The increase of plasma PAI in hyperinsulemic rabbits might therefore be caused by an indirect effect of insulin on endothelial cells or by an increased PAI-1 synthesis by other cell types such as liver cells. 
Influence of hyperlipoproteinemia on the fibrinolytic system and the clotting system in patients with and without angiographically proven coronary artery disease

M. Heins, W. Withold, F.-C. Schoebel*, H. Dauwitz, K. Pels*, D. Stein*, M. Friebe*, M. Leschke*, B. E. Strauer*, H. Reinauer

Institut für Klinische Chemie und Med. Klinik und Poliklinik B*, Heinrich Heine Universität Düsseldorf

Hyperlipoproteinemia is considered as a major cause for artherosclerosis and coconary artery disease (CAD). For the determination of the effect of hyperlipoproteinemia on the fibrinolytic system and on the activation of the clotting system cholesterol (CHOL), triglycerides (TG), the concentration of whole tissue plasminogen activator ( $\mathrm{t}-\mathrm{PA}$ ), the activity of plasminogenactivator-inhibitor (PAI) and the concentration of prothrombinfragment $1+2(\mathrm{~F} 1+2)$ were analysed in 225 consecutive patients. Of these 225 patients 66 had a TG and a CHOL concentration of more than $200 \mathrm{mg} / \mathrm{dl}$ and were defined as high risk group (HR). These parameters were also determined in the other 159 patients. The distribution of age as well as the distribution of patients with HMG-CoA-reductase inhibitor (HR $30 \%$ versus NR $31 \%$ ) and fibrate therapy (HR $11 \%$ versus NR 6\%) were similar in both groups.

In HR patients as compared to controls significantly increased concentrations (activities) of t-PA (HR: $9[6-14] \mathrm{ng} / \mathrm{ml}$; NR $8[4-11] \mathrm{ng} / \mathrm{ml}$ (Median [10. - 90. Percentil]); $\mathrm{p}<0,01$ ) and PAI (HR: 5,5 $[2,7-11,2] \mathrm{U} / \mathrm{ml}$; NR $3,6[1,8-8,2] \mathrm{ng} / \mathrm{ml}$ (Median [10. - 90. Percentil]); $p<0,001$ ) were found. A significant difference could not be detected in the extend of CAD. The percentage of patients with CAD was essentially the same in both groups (HR $64 \%$, NR $61 \%)$.

Conclusion: Our study shows that in high risk patients as compared to a control collective of patients PAI significantly increased, whereby this effect is largely compensated by an activation of fibrinolysis via t-PA. In this cohort differentation of patients into high risk groups according to lipid profile did not distinguish to the prvalence of $\mathrm{CAD}$. Nevertheless these results indicate an interaction between lipidmetabolism and fibrinolysis implicating new aspects in lipidloweringtherapy in patients with $\mathrm{CAD}$.

FIBRINOLYTIC PARAMETERS AT BASAL CONDITIONS AND AFTER VENOUS OCCLUSION AND EXERCISE STRESS TEST IN YOUNG PATIENTS WITH CORONARY ARTERY DISEASE

S. Graf, R. Beckmann. J. Koller, G. Christ, B. R. Binder and K. Huber

Venous occlusion (VO) and exercise stress test (EST) are known to stimulate the fibrinolytic potential in healthy induviduals wereas this is not the case in a significant percentage of patients (pts) with thrombesis associated diseases. Wo investigated $26 \mathrm{pts}$ ( $M / F=20 / 6$; mean age: $38.6 \pm 6$ ) with angiographically proven coronary artery disease $(C A D)$ for their plasma levels of tissue plasminogen activator ( $t-P A)$, plasminogen activator inhibitor $-1(P A \mid-1)$ and urokinase-type plasminagen activator $(U-P A)$ before and after VO and EST and compared the data to those obtained from 16 healthy sex- and age-matched controls (ctr). At basal conditions, maan plasma lovols of $t-P A$ activity and antigen as well as 1 -PA antigen were nol different between the two study groups. PAI- 1 activity plasma lovels were significantly inereesed in pts as compared to ctr ( $p<0.01)$ which was not the case for PAI-1 antigon levols. After VO, mean t-PA activity and antigen lovels increased to a significant higher extent in the control group ( $p<0.01$ ) whilo $u-P A$ antigen levels were unaltered. The different increase of $t-$ $P A$ levels in the two study groups can be explained by the individual bohaviour of t-PA, which was not stimulated by Vo in pts. in $66.7 \%$ (activity) and $50 \%$ (antigen) as compared to $25 \%$ (activity) and $21.4 \%$ (antigen) in ctr, respectivaly. PAI-1 activity levels showed in both groups a significant decrease (pts: $-4.4 \pm$ 0.7 ; ctr: $-4.5 \pm 1.0 ; \mathrm{U} / \mathrm{ml}$; p 0.01 ), whilo PAl-1 antigen lovels increased in both groups slightly but not significantly. At the time of maximal physical exercise (bycicle EST) we tound a significant higher increase of $t-P A$ activity in ctr $(+4,4 \pm 1,5 \mathrm{U} / \mathrm{ml})$ as compared to pts $(+0,49 \pm 0,2 \mathrm{U} / \mathrm{ml})(p<0,02)$. Howover, $t-P A$ antigen did not increase to a different extent in the study groups ( $3.1 \pm 1$ $\mathrm{ng} / \mathrm{m} /$ each). Similar to VO, EST did not load to significant changes in U -PA antigen levels. PAI- 1 activity levels docr rased and PAI-1 antigen levels increased due to EST without any significant difference between the study groups. Wo conclude from these chata, that the determination of $P A \mid-1$ activity at rest and of $t-P A$ antigen and activity befors and after VO or before and at maximal work load during EST might be of clinical value for the detection of a defective fibrinalytic system in CAD pts whilo PAI- 1 antigen and $U-P A$ antigen determinations are of less value.

$2^{\text {nd }}$ Department of Internal Medicine, Division of Cardiology, University of Vienna, Währinger Gürtel 18-20, A-1090 Vienna, Austria

\section{PLASMINOGEN ACTIVATOR INHIBITOR 1 (PAI-1) IN NORMAL AND ATHEROSCLEROTIC HUMAN ARTERIES}

J.Kienast, M.Steins, T. Padro

A quantitative analysis of the content and activity as well as layer distribution of PAl-1 in nomal and atherosclerotic (AT) human arteries is reported. PAI- 1 antigen and activity were determined in protein extracts of intima and media layers of human aorta and coronary arteries. Crosssections of the arteries were classified as areas without atherosclerotic lesions (NL), with early lesions (EL), fibrous plaques (FP) and advanced lesions (AL).

Normal arteries: PAl-1 antigen levels were higher in aortic vessel wall as compared to coronary arteries $(13.5 \mathrm{ng} / 100 \mathrm{mg}$ wet tissue (wt) in aortic intima, $23.8 \mathrm{ng} / 100 \mathrm{mg}$ wt in aortic media versus $3.5 \mathrm{ng} / 100 \mathrm{mg}$ wt in coronaries). In aorta, the difference in PAl-1 antigen levels between intima and media layer was significant $(p<0.001)$ with a similar difference in extractable PAl activity (on average $3 \times$ higher in media). Active PAl could not be extracted from coronary arteries.

Atherosclerotic vessel wall: PAl-1 antigen strongly increased in AT segments in direct relation to the severity of the lesions: $E L \times 3$; FP $\times$ 11; AL $\times 12$. The increase was more pronounced in intima than in the media layer with the highest PAI-1 antigen levels being detected in the core region of FP. Despite increasing antigen levels, no free activity was extracted from AT segments of coronary arteries. However, the presence of active PAl in vivo could be inferred from the detection of t-PA:PAl-1 complex in coronary extracts with $\mathrm{FP}$ and $\mathrm{AL}$ containing significantly higher levels than NL and EL segments. Furthermore, PAl-1 antigen and t-PA:PAl-1 complex were positively correlated in AT coronary arteries $(r=0.55 ; p<0.01)$.

The data suggest an involvement of the plasminogen activator / plasmin system in atherogenesis with a regulatory role for PAl-1.

Department of Internal Medicine, University of Münster, AlbertSchweitzer-Straße 33, 48129 Münster 
12

\section{RECENT TRIALS OF THROMBOLYTIC TREATMENT IN PATIENTS WITH ACUTE MYOCARDIAL INFARCTION}

W. Rutsch

Several large trials in acute myocardial infarction have demonstrated that thrombolytic therapy improves survival. There continues to be substantial refinement in the approach to the patient with myocardial infarction. Reduced mortality may be related to early thrombolysis, limitation of infarct size and preservation of left ventricular function. The GUSTO trial enrolled 41,021 patients into four arms of therapy: (1) accelerated alteplase t-PA and iv heparin; (2) combination t-PA with streptokinase and iv heparin; (3) streptokinase with iv heparin; or (4) streptokinase with high-dose sq heparin. Accelerated t-PA with proved to be the winning strategy, leading to 14 percent mortality reduction. The angiographic substudy showed for the first time a 40 percent advantage for 90 minute patency (TIMI 2 or 3) for accelerated t-PA versus the SK strategies, a near 60 percent increase in TIMI-3 patency, and a corresponding 20 percent decrease in mortality in the overall trial by 24 hours. Three recent randomized trials of pre hospital thrombolysis have emphasized the value of very early administration of therapy in the course of the event. The Myocardial Infarction Triage and Intervention (MITI) trial did
not show an advantage of pre hospital thrombolysis compared with in-hospital treatment with respect to infarct size or ejection fraction. The largest trial of pre hospital thrombolysis was the European Myocardial Infarction Project (EMIP), which enrolled approximately 6,000 patients. Although the statistical comparison indicates the difference is not significant at the $p<0,05$ level, cardiac mortality was significantly reduced. The Early Treatment Grampian (GREAT) trial also conferred the advantage of pre hospital therapy. In a relatively small trial like MITI, there was a benefit in cardiac function and infarct size assesses via serial enzymes. Two recent mortality reduction trials have advanced our understanding as to how long the therapeutic window is for the benefits of thrombolytic therapy. The TAMI- 6 trial enrolled 200 patients who presented 6 to 24 hours after symptom onset and were randomly assigned to t-PA or placebo. This trial showed that t-PA lysed relatively aged thrombus efficiently and that this was associated with inhibition of cavity dilation, albeit without any evidence for ejection fraction ciated with inhibition of cavity dilation, albeit without any evidence for ejection fraction
improvement. The Late Assessment of Thrombolytic Efficacy (LATE) trial enrolled more than 5,700 patients presenting between 6 and 24 hours after symptom onset for randomisation between E-PA or placebo. There was no benefit for patients treated in the 12- to 24 hour time window. The second late entry trial was the Estudios Multicentrico Estreptoquinasa Republica Americas Sud (EMERAS), which enrolled 4,534 patients. Although this study did not find a statistically significant benefit of patients treated in the 6 - to 12 hour time frame, there was a trend with a 12 per cent reduction in mortality. The meta-analysis of the Fibrinolytic Therapy Trialist's Collaboration, which systematically pooled data from 52,892 patients enrolled into eight placebo-controlled trials, showed significant benefit out to 12 hours, but not beyond this time point.

Department of Cardiology, Freie Universität Berlin, Klinikum Rudolf-Virchow, Spandauer Damm 130, 14050 Berlin-Charlottenburg, Germany.

13

CHRONIC-INTERMITTENT UROKINASE THERAPY IN REFRACTORY ANGINA PECTORIS: LONG TERM FOLLOW-UP IN 121 PATIENTS

Leschke, M., F.-C. Schoebel, B.E. Strauer - Med. Klinik and Poliklinik B,

Heinrich Heine University, Düsseldorf, Germany

Chronic-intermittent urokinase therapy (UK-therapy) as a new approach to therapy-refractory angina pectoris in coronary artery disease (CAD) was to be evaluated for long-term follow up in 121 patients (pat.).

All pat. had severe coronary artery disease without a favourable option for interventional revascularisation and were refractory to maximal conventional medical combination therapy (nitrates, beta-blockers, calcium antagonists). UK was administered as bolus injection $3 \times 500000 \mathrm{U}$ i.v./ week over a period of 3 months.

After 3 months fibrinogen levels $(375 \pm 91$ to $238 \pm 32 \mathrm{mg} / \mathrm{dl}$; $p<0.01)$ and subsequently plasma viscosity $(1.38 \pm 0.07$ to $1.32 \pm 0.07$ $\mathrm{mPas}$ ) were reduced significantly. Incidence of daily anginal episodes were reduced (by $66 \%$ from $3.4 \pm 1.7 \quad / d ; p<0.001$ ) as well as intake of fast acting nitrates (by $70 \%$ from $3.3 \pm 1.9$ capsules/d; $p<0.001$ ). Myocardial perfusion as documented by Tc $99 \mathrm{~m}$ MIBI-SPECT analysis increased globally by $21 \%(p<0.01)$. During the follow-up period (18.5 \pm 10 months) clinical improvement lasted for $12.7 \pm 8.5$ months; in $31 \%$ refractory angina recurred. In this high risk group coronary mortality was $12 \%$. Clinical admissions for cardiovascular events (unstable angina, myocardial infarction) as compared to the period 12 months prior to UK-therapy were significantly reduced (65 to 35 , $\mathrm{p}<0.01$ ).

Conclusion: Chronic intermittent UK-therapy as a rheological and possibly fibrinolytic approach represents a new effective form of treatment for pat. with refractory angina pectoris due to improvement of myocardial perfusion. Long term results demonstrate cost-effectiveness regarding costs for medical treatment as well as quality of life and prognosis for survival.
14

AN ACCELERATED "FRONTLOADED" RT-PA THROMBOLYSIS REGIMEN ACHIVES HIGHER TIMI III PATENCY RATES IN ACUTE MYOCARDIAL INFARCTION

D.C. Gulbal ${ }^{1 \& 2}$, S. Hauck ${ }^{2}$, R. Dechend ${ }^{2}$, K. -J. Osterziel ${ }^{2}$, M. Barthels', P.R. Lichtlen ${ }^{1}$. R. Dietz ${ }^{2}$. Medizinische Hochschule Hannover (1) \& UKRV Franz-Volhard-Klinik. Berlin (2)

It has been demonstrated, that post AMI prognosis is improved only when prompt coronary blood flow (CBF) (TIMI grade III) is achieved. However even with the "frontloaded" rt-PA regimen. designed by Neuhaus. TIMI III CBF is achived in only $56 \%$ of the patients (pts) 90 min into thrombolysis (T) (GUSTO-study). In an angiographic dose ranging study perfusion rates with different $T$ regimen were investigated. $4 \times 20$ pts were enroled. (A: 2.25 MIO IE SK/60 min: B: $70 \mathrm{mg}$ rt-PA/90 min: C: $100 \mathrm{mg} \mathrm{rt}-\mathrm{PA} / 90 \mathrm{~min} ; \mathrm{D}: 100$ mg $\mathrm{tt}-\mathrm{PA} / 60 \mathrm{~min})$

\begin{tabular}{|c|c|c|c|c|c|c|c|}
\hline regimen & male & female & post. MI & Lat. MI & ant. MI & TIMI II & TIMI III \\
\hline A & 18 & 2 & 8 & 3 & 9 & $3(15 \%)$ & $7(35 \%)$ \\
\hline B & 16 & 4 & 10 & 4 & 7 & $4(20 \%)$ & $9(45 \%)$ \\
\hline C & 17 & 3 & 9 & 3 & 8 & $3(15 \%)$ & $12(60 \%)$ \\
\hline D & 14 & 6 & 11 & 2 & 7 & $1(5 \%)$ & $16(80 \%)$ \\
\hline
\end{tabular}

The favourable results of regime $D$ were further studied in a consecutive series of 163 pts.

\begin{tabular}{|c|c|c|c|c|c|c|c|}
\hline regimen & male & female & post. MI & lat. MI & ant. MI & TIMI II & TIMI III \\
\hline $\mathrm{D}$ & 126 & 37 & 74 & 68 & 21 & $11(6.8 \%)$ & $131(80.3 \%)$ \\
\hline
\end{tabular}

During $T$ with regimen $D$ fibrinogen and plasminogen levels dropped on average $0,8+1-0,6 \mathrm{~g} / \mathrm{l}$ and $52+/-30 \%$ respectively. Severe bleeds were observed in only $5.5 \%$ of pts.

From these results we conclude that improved TIMI III CBF may be achived by the accelerated "frontloaded" rt-PA regimen D. With this regimen the rate of side effects virtually remains the same as compared to standard treatments including the Neuhaus regimen.

present address: Klinikum Rudolf Virchow. FU Berlin, Franz-Volhard-Klinik \& Max Delbrück Centrum für molekulare Medizin. Wiltbergstr.50. 13125 Berlin

\section{Local application of plasminogen activators in patients with peripheral occlusive disease Spengel F. A.}

Efficacy and safety in local thrombolytic therapy has been demonstrated for streptokinase- and urokinase-type plasminogen activator. With the availabitity of recombinant tissue-type plasminogen activator high local thrombolytic efficacy could be shown using total amounts below $10 \mathrm{mg}$. Lowering the total dose applied results in optimal safety of the recombinant plasminogen activator.

Local application of plasminogen activators in 900 patients was accompanied by a $0.2 \%$ mortality. Mean age of patients was 74 years. Recanalization rates of more then $90 \%$ have been demonstrated in peripheral arterial occlusions (4-22 $\mathrm{cm}$ long, 5-160 days old). No significant differences in patency rates were detected between patients with thrombotic or embolic occlusions. Mechanical devices for removal of occluding material were necessary in $40 \%$ of the occlusions.

To improve recanalization and patency rates the application of the thrombolytic compound must be accompanied by antiplatelet and anticoagulant therapy.

Local thrombolysis is a save and effective method, to reopen occluded sections of the superficial femoral and popliteal artery and of calf arteries.

Medizinische Poliklinik, Abteilung Angiologie, Klinikum Innenstadt der Universität München 
16

\section{FRONTLOADING FIBRINOLYTIC THERAPY WITH RT-PA IN BRANCH AND CENTRAL RETINAL VEIN OCCLUSION}

\section{L.-O. Hattenbach, G.W.K. Steinkamp, Chr. Ohrloff ${ }^{1}$, W. Mondorf, I. Scharrer 2}

Although central retinal vein occlusion and branch vein occlusion are among the most common vascular disorders affecting the retina, much confusion exists regarding their management.

In view of the pathophysiologic features of CRVO, the use of fibrinolytic agents such as rt-PA (recombinant tissue-plasminogen activator) appears to be the most promising therapeutic approach.

Patients:

In 16 patients with ischemic retinal vein occlusion, $50 \mathrm{mg}$ of $\mathrm{rt}$-PA were given intravenously over a period of 60 minutes. Simultaneously, heparin was infused at 1200 units/hour. The i.v. heparinization was continued over a period of 8 days.

Results:

The clinical course was assessed by documenting visual acuity, fundal examinations and fluorescein angiography.

14 patients showed an improvement in visual acuity and retinal perfusion. No hemorrhagic complications were noted.

Conclusion:

Based on our results, we would recommend the frontloading fibrinolytic therapy with rt-PA and heparin in the treatment of ischernic retinal vein occlusions. For a final assessment, further investigation and comparative studies will be required.

${ }^{1}$ Zentrum der Augenheilkunde; ${ }^{2}$ Abteilung für Angiologie, Zentrum der Inneren Medizin; Klinikum der Johann Wolfgang Goethe-Universität, Frankfurt am Main, Germany

\section{$16 a$}

INHTBITION OF ADP-INDUCED PLATELET ACTIVATION BY TICLOPIDINE AND CLOPIDOGREI

\section{K. Schrör}

Ticlopidine and clopidogrel are two thienopyridines which cause an irreversible inhibition of platelet function. These antiplatelet effects are mainly directed against ADP-induced stimulation of platelet function, specifically, ADPinduced inhibition of adenylyl cyclase stimulation via the 2-methylthio-ADP-type binding site of ADP at the platelet membrane. There is evidence for additional effects of thienopyridines, including inhibition of agonist-induced intracellular $\mathrm{Ca}^{++}$mobilization, interference with fibrinogen receptor/agonist interaction and inhibition of platelet $\alpha$-granule secretion. However, these actions are probably secondary to the ADP-antagonistic effects.

Thienopyridines do not dixectly interfere with arachidonic acid metabolism. The substances are inactive in vitro and have to undergo a bioactivation in vivo. This requires 3-5 days of treatment for a maximum effect. The nature of the postulated active metabolite(s) is still unknown. From a pharmacological point of view, thienopyridines may be considered interesting alternatives to acetylsalicylic acid with particular value in shear-stress-mediated platelet activation in vessel stenoses associated with endothelial injury.

Institut für pharmakologie, Heinrich-Heine-Universität Düsseldorf, Moorenstr. 5, 40225 Düsseldorf

\section{Platelets I+ II}

17

Changes in Platelet Membrane Glycoproteins in Patients with Sepsis and Multiple Organ Failure

M. Gawaz ${ }^{1}$, S. Fateh-Moghadam ${ }^{2}$, G. Pilz ${ }^{2}$, H.-J. Gurland ${ }^{2}$, K.Werdan ${ }^{2}$

Sepsis and multiple organ failure (MOF) is associated with multiple changes in hemostatic mechanisms. To study the role of platelet activation in patients with sepsis and multiple organ failure (MOF), we examined surface expression of adhesion molecules on circulating platelets of 14 intensive care patients with suspected sepsis and MOF. Severity of disease was assessed by Elebute (sepsis) and APACHE II (MOF) scoring systems, respectively. Using flow cytometric techniques and monoclonal antibodies, surface expression of GPIIb-IIIa, GPIb, GMP-140, GP53, LIBS1, and thrombospondin was measured. Receptor density of GPIIb-IIIa and GPIb on circulating platelets was not affected by sepsis or MOF. In septic patients surface expression of LIBS1 was significantly elevated $(p<0.05)$ and correlated well with severity of disease $(r=0.597)$. No significant change in granule glycoprotein expression (TSP, GMP140, GP53) was noted in septic patients. In contrast, degranulation of granule glycoproteins was significantly elevated in MOF $(p<0.05)$ that correlated well with severity of MOF $(r=0.643)$. We speculate that platelets in sepsis circulate in a hyperaggregable state that results in increased risk of microthrombotic events. In the course of the disease, irreversible platelet degranulation might occur and may play a role in development of MOF in these patients.

1I. Medizinische Klinik, Klinikum Rechts der Isar der TUM and 2Medizinische Klinik I, Klinikum Großhadern der LMU, München, Germany
18

ALPHA AND DENSE GRANULA SECRETION OF GLYCOPROTEIN HIB (GPIIIB, GPIV, CD36)-DEFICIENT PLATELETS

B.Kehrel, U.Niehues, A.Kronenberg, J.Kardoeus, K.J.Clemetson

GPIIlb has been reported to be a receptor for collagen, thrombospondin, plasmodium falciparum-infected erythrocytes and very recently for oxidized low density lipoprotein.

To further elucidate the physiologic roles of platelet GPIIlb we compared the secretion of GPIIIb-deficient platelets to that of GPIIIbpositive control platelets. Blood donors with GPIllb-deficient platelets have been described by us previously.

$P$-selectin (GMP-140, CD62) expression as an indirect indicator of $\alpha$ granula secretion of thrombin-stimulated GPIllb-deficient platelets and control platelets was followed up by flow cytometry using antibody CLBthromb/6. The kinetic analysis of $\alpha$-granule secretion (PF4, BTg) was performed according to Akkerman et al (1982). The secretions were induced by addition of collagen type $\mathrm{I}$, $\mathrm{III}$ or $\mathrm{V}$ and stopped with formaldehyde. PF4 and $\beta \mathrm{Tg}$ were assayed using ELISA kits. ATP release was monitored on a lumi-aggregometer by a luciferin-luciferase assay.

GPIllb-deficient platelets responded with normal ATP release to collagens type I and III. Secretion of $\alpha$-granule from GPIIIb-deficient platelets induced by type $\mathrm{I}$ and III collagens studied by measuring the release of $\beta T g$ and PF4 into the plasma did not differ from normal platelets. In accordance with the aggregation results both ATP and $\alpha$ granule secretion of GPIllb-deficient platelets in response to type $V$ collagen was significantly impaired. No difference was observed in P. selectin expression kinetics between GPIIlb-deficient and control platelets.

The results indicate that GPIIlb-deficient platelets show normal $\alpha$-and dense granule secretion, suggesting that the presence of GPIIIb is not required for normal platelet secretion.

Haemostasis Research Group, Department of Internal Medicine, University of Münster, Domagkstr.3, D-48129 Münster, Germany 
EFFECT OF PALLIDIPIN ON HUMAN PLATELET AGGREGATION

Ch. Noeske-Jungblut, A. Becker, P. Verhallen, P. Donner and W.-D. Schleuning

Pallidipin, a $19 \mathrm{kDa}$ protein from the saliva of the bug Triatoma pallidipennis, is a specific inhibitor of collagenmediated platelet aggregation. The effect is dose-dependent with an IC 50 of $150 \pm 100 \mathrm{nM}$. Aggregation induced by other effectors, e.g. ADP, thrombin or U46619, was not inhibited. Increasing amounts of collagen were able to overcome the inhibition of aggregation by pallidipin. No effect was noted on platelet adhesion to a collagen matrix in a static model even at higher concentrations $(2 \mu \mathrm{M})$ while a monoclonal antibody directed against glycoprotein la/la, a collagen receptor on platelets, blocked the adhesion in the same assay. Platelet shape change was not totally inhibited by pallidipin. In contrast, coliagen-induced ATP secretion was prevented. These results suggest that pallidipin exhibits its inhibitory effect by a reversible kind of interaction with the platelets. However, the precise mechanism of action remains to be investigated.

Research Laboratories of Schering AG, D-13342 Berlin, Germany

\section{Contact Phase}

20

CONTACT PHASE AND FIBRINOLYSIS

G. Dooijewaard, Gaubius Laboratory PG-TNO, P.o. Box 430, 2300 AK Leiden, The Netherlands

In this presentation the view is put forward that fibrinolysis is a natural defence mechanism against thromos, which comprises cells of the vessel wall (cellular system) and the action of $a$ cascade of proteolytic proenzymes and enzymes in the circulation (humoral system). The cellular system provides the first defence against undue fibrin deposition: it sets the selectivity (intact vessel wall) and specificity (fibrin) of the fibrinolytic process, and initiates the process by constitutive secretion of $t-P A$. The humoral system provides the second defence: it accomplishes an amplification of the fibrinolytic process by accelerating the plasmin generation. Many proenzymes from the blood are potentially involved in the cascade, notably scu-PA, the contact system component factor XII and prekallikrein, and contact system components factor XII and prekallikrein, and a third plasminogen proactivator. since for all the activated proenzymes potent inhlbitors are present in the blood stream, the cascade process can only effectively proceed at the surface of the by fibrin and blood platelets covered vessel wall. Specific receptors for kininogen and scu-PA on blood platelets and the endothelial cells of the vessel wall aid to focalize the process. The potential impact of the cascade is great, the more since the hormone bradykinin is concomitantly set free from kininogen and may induce acute release of $t-p A$ from the vessel wall, on top of the constitutive secretion.

There is good evidence that in individuals at risk for thrombosis parts of the vessel wall are degenerate, resulting locally in an impaired secretion of t-pa, leading to to steadily growing, undue, fibrin-containing deposits (thrombi) compensation of the local fibrinolytic defect by systemically reinforclng the second defence (larger amplification factor), seems to be a straightforward prophylactic measure that may delay the fatal, occluding thrombosis. The plans of an international concerted action (ECAPTURE) to approve that this approach of prevention from thrombosis will be beneficial and feasible, are unfolded. ECAPTURE, the European Concerted Action on Prevention from Thrombosis by Urokinase Enhancement, is sponsored by grant BMHl-CT92-0392 from the commission of the European Comrnunities, Brussels.
21

IS FACTOR XII DEFICIENCY A THROMBOPHILIC STATE?

B. Lämmle, J. Kremer Hovinga, and M. Furlan

There is considerable debate in the literature on the importance of coagulation factor XII ( $F$ XII) deficiency as a thrombophilic state. Several anecdotal case reports suggest that severe F XII deficiency may be associated with an increased risk for thromboembolic complications probably due to impaired intrinsic fibrinolysis.

Our group performed two studies in order to address the question whether F XII deficiency is a strong and/or frequent prethrombotic risk factor.

In a first study (Thromb. Haemostas. 1991; 65:117) 74 subjects from 14 families with known $F$ XII deficiency were investigated in a cross-sectional study. Whereas two out of 18 subjects with $F$ XII:C $<0.01 \mathrm{U} / \mathrm{ml}$ had suffered from venous thromboembolism at age $<40$ years, only one out of 45 subjects with partial F XII deficiency (definite or probable heterozygous $F$ XII deficiency) had had a possible venous thrombosis. Heterozygous F XII deficiency, therefore, is not a strong risk factor for thromboembolism.

In a second study (Blood Coag. Fibrinol. 1992; 3:555), F XII levels in 200 subjects having suffered from venous and/or arterial thromboembolism were compared with those in 200 healthy controls. Low $F X I I: C$ and $F X I I: A g$ values were not more common in thrombophilic patients than in controls, suggesting that partial $F$ XII deficiency is not a very frequent prethrombotic risk factor.

Whether partial F XII deficiency disposes elderly patients to thromboembolic complications awaits further study.

Central Hematology Laboratory, Inselspital, University Hospital Bern, CH-3010 Bern, Switzerland 
22

F XII DEFICIENCY AMONG 300 HEALTHY BLOOD DONORS

W. - M. Halbmayer 1, A. Haushofer ${ }^{1}$, R. $\operatorname{Schön}^{1}$, C. Mannhalter ${ }^{2}$, E. Strohmer ${ }^{3}$, K. Baumgarten 3 and $M$. Fischer ${ }^{1}$.

Factor XII (F XII) deficiency has been reported to be a risk factor for the development of arterial and venous thromboembolism. However, no data are available on the prevalence of F XII deficiency within the normal population. Measuring APTT and F XII activity, seven F-XII deficiencies could be detected among 300 healthy blood donors. This corresponds to an incidence of F XII deficiency of $2.3 \%$. On the basis of these data the prevalence of severe and mild F XII deficiency in the normal population can be estimated to be $1.5-3.0 \%$. Assessment of F XII antigen levels revealed, that all seven F XII deficient individuals had F-XII antigen levels matching the activity. One presented a severe $F$ XII deficiency $(1 / 300,0.3 \%)$ without detectable $F$ XII activity and an APTT prolongation of more than 120 seconds. The remaining six $F$ XII deficiencies $(6 / 300,2.0$ क) were moderate variations with $F$ XII activities ranging from 20-45\% and less prolonged APTTs.

Icentral Laboratory, General Hospital ViennaLainz, Wolkersbergenstraße 1, A-1130 Vienna, Austria. ${ }^{2}$ Clinical Institute of Medical and Chemical Diagnostics, University Vienna and ${ }^{3}$ Blood Donation Center, Red cross, vienna, Austria.
Aprotinin inhibits the contact phase of coagulation during cardio-pulmonary-bypass in man and has anticoagulatory properties

Authors: W. Dietrich,M.D., M. Spannnagl, M.D., J.A. Richter, M.D.

Several studies ${ }^{1-3}$ have demonstrated the reduction of bleeding tendency during and after cardio-pulmonary-bypass (CPB) due to the proteinase inhibitor aprotinin. One possible mechanism of its action is the inhibition of contact activation during cardio-pulmonary bypass. Methods: With institutional approval and informed consent 40 male patients undergoing myocardial revascularization were enrolled in this double-blind study. Patients were randomly assigned to one of two groups: group $A(n=20)$ received high dose aprotinin during operation, patients of group $C(n=20)$ placebo. Anticoagulation was achieved with $375 \mathrm{U}$ mucosa heparin with control of celite activated ACT. Blood samples were taken at preset points. F1/F2 prothrombin fragments, the thrombin antithrombin III complex (TAT), fibrin monomeres were determined by ELISAs using polyclonal and monocional antibodies. Intra- and postoperative blood loss was recorded. ANOVA was used for statistics.

Results End of CPB

$\begin{array}{lcccc} & \begin{array}{c}\text { F1/F2 fragments } \\ {[\mathrm{ng} / \mathrm{mL}]}\end{array} & \begin{array}{c}\text { TAT } \\ {[\mathrm{ng} / \mathrm{mL}]}\end{array} & \begin{array}{c}\text { fibrin } \\ {[\mu \mathrm{m} / \mathrm{mL}]}\end{array} & \\ \text { group C } & 6.6 \pm 2.8 & 76.4 \pm 18.6 & 16.0 \pm 12.7 & \\ \text { group A. } & 3.5 \pm 0.8 & 48.8 \pm 12.4 & 4.2 \pm 3.0 & (p<0.05)\end{array}$

ACT was significantly increased in group A. Bleeding tendency and homologous blood requirement was significantly reduced in group $A$. Discussion: The results of this study indicate a significantly reduced clotting activation due to aprotinin. Aprotinin attenuates prothrombin conversion to thrombin and has a synergistic anticoagulatory effect to heparin. Clotting activation is diminished via inhibition of the intrinsic pathway. This mechanism is responsible for better preserved hemostasis after CPB in open heart surgery.

References: 1. Blauhut B et al . J Thorac Cardiovasc Surg ; 101:958,1991

2. Dietrich $W$ et al...Anesthesiology $73: 1119,1990$

3. Royston D et al.Lancet; ;ii:1289, 1987
PLASMA KALLIKREIN INHIBITION AND B2 KININ RECEPTOR BLOCKADE IN EXPERIMENTAL CONTACT SYSTEM ACTIVATION

M Siebeck, J Cheronis, E Fink, M Spannag1, H Fritz, W Schramm

Dextran sulfate (DXS) activates the contact system and, in vivo, produces transient hypotension. In order to better define the mechanisms underlying the DXS-induced hypotension, we investigated the effects of either the plasma kallikre in inhibitor, des-Pro2-[Arg15]-aprotin in (BAY 4620) or the B2 kin in antagonist, Hoe 140 on the hypotensive response to DXS. In the first study, anesthetized miniature pigs (5 pigs/group, randomly assigned) were given one of the following treatment protocols: 1) DXS (5 mg/ $/ \mathrm{kg}), 2-5)$ DXS plus BAY $4620(45,90,180$, or $360 \mathrm{mg})$, or 6$)$ saline. DXS a lone produced a profound but transient systemic arteria 1 hypotension with a corresponding reduction in plasma kinincontaining kininogen. Circulating $k$ in in levels, complement fragment C3adesArg and fibrin monomer were all increased. BAY 4620 produced a dose-dependent de lay or attenuation in these effects with the highest dose completely blocking DXSinduced hypotension and elevations of $k$ in in, C3adesArg and fibrin monomer levels. Thus, the effects of DXs are solely dependent on contact system activation and this activation is sensitive to BAY 4620 . However, contact system activation is known to produce changes in a variety of vasoactive mediators, all of which can affect blood pressure. In a second study, two groups of pigs (3/group) were given either DXS a lone $(2 \mathrm{mg} / \mathrm{kg})$ or DXS 10 minutes after a bolus injection of Hoe $140(30 \mu \mathrm{g} / \mathrm{kg})$. DXS a lone produced transient hypotension. This response was completely blocked by Hoe 140 pretreatment. Both groups had identical reductions in $k$ in incontaining kininogen. We conclude that DXS-induced hypotension is produced by activation of the contact system which results in the production of bradykinin. Liberation of bradykinin is both necessary and sufficient to produce all of the hemodynamic changes observed.

Department of Surgery, University of Munich, Klinikum Innenstadt, Nussbaumstrasse 20, D-80336 Munich, GERMANY
Antifibrinolytic effects of aprotinin in patients undergoing cardio-pulmonary bypass surgery Spannagl M, Dietrich W, Dooijewaard G, Kluft C, Schramm W

Intraoperative high-dose aprotinin administration has been shown to reduce the intra- and postoperative blood loss in cardiac surgery. The haemostatic effect has been attributed to platelet preserving properties and to attenuation of hypercoagulability and hyperfibrinolysis during and after cardiopulmonary bypass (CPB).

We investigated the effects of aprotinin on fibrinolytic activity measuring degradation products of fibrinogen and fibrin, antiplasmin - plasmin complex, tissue- and urokinase-type plasminogen activator activity and antigen in plasma of twenty male patients undergoing myocardial revascularization randomly assigned to aprotinin (A) or control group (C). Plasma parameters were measured at different stages intraoperatively and two hours postoperatively. The increase in degradation products during and after $\mathrm{CPB}$ was significantly reduced in $A$ as compared to $C$. After an initial drop due to haemodilution at the onset of CPB, levels of free t-PA and u$P A$ restored intraoperatively in $A$, but remained subnormal in $C$ until the end of the observation period.

It is concluded, that with aprotinin administration activation of fibrinolysis during CPB is significantly reduced. The effect is due to inhibition of intrinsic and extrinsic fibrinolysis and results in reduced bleeding consequences.

Medizinische Klinik und Deutsches Herzzentrum München, Gaubius Laboratory, IVVO-TNO, Leiden 
26

\section{Aprotinin Inhibits Coagulation and Fibrinolysis during CPB in Patients with Reduced Heparin Response}

G. Dilthey, M.D.; W. Dietrich, M.D.; M. Spannagl, M.D.; M. Jochum Ph.D.; J. A. Richter, M. D.

Introduction. Intraoperative aprotinin treatment reduces bleeding tendency in cardiac surgery. Patients on heparin treatment often show a reduced response to heparin and activation of coagulation during cardiopulmonary bypass (CPB), followed by increased postoperative bleeding. We postulate that aprotinin acts synergistically with heparin and may lead to improved anticoagulation during CPB and reduced postoperative blood loss.

Methods. Thirty patients preoperatively treated with heparin for at least 10 days and scheduled for coronary artery bypass grafting were studied. Patients randomly received either a bolus of $2 \times 10^{6} \mathrm{KIU}$ of aprotinin after induction of anesthesia and at the start of CPB as well as a continuous infusion of $5 \times 10^{5} \mathrm{KIU} / \mathrm{h}$ during the entire operation, or saline. Plasma levels of AT III, heparin, thrombin-antithrombin III complex (TAT), fibrin monomers (FM) and D-dimers were investigated at preset time points. ANOVA was used for data analysis.

Besults. Preoperative ATIII was $74 \pm 10 \%$ (control) and $73 \pm 15 \%$ (aprotinin). Parameters of coagulation and fibrinolysis were significantly reduced in aprotinin treated patients. At the end of CPB, FM were $32.7 \pm 9.0 \mu \mathrm{g} / \mathrm{mL}$ (control) and $19.4 \pm 11.3 \mu \mathrm{g} / \mathrm{mL}$ (aprotinin), $p<0,05 ;$ and TAT was $74.0 \pm 13.3 \mu \mathrm{g} / \mathrm{L}$ and $57.2 \pm 18.4 \mu \mathrm{g} / \mathrm{L}$, respectively $(p<0.05)$. At the same time point, DD were reduced to $1.6 \pm 2.1 \mu \mathrm{g} / \mathrm{mL}$ (aprotinin) compared to $5.8 \pm 2.4 \mu \mathrm{g} / \mathrm{mL}$ (control), $p<0,05.24 \mathrm{~h}$ postoperative blood loss was $1469 \pm 665 \mathrm{~mL}$ (control) and $689 \pm 327 \mathrm{~mL}$ (aprotinin), $p<0.05$.

Discussion. Patients on heparin treatment are prone to the risk of heparin resistance. Aprotinin treatment in these patients attenuates activation of clotting and fibrinolysis. Aprotinin improves anticoagulation via inhibition of contact phase activation. This improved anticoagulation during $\mathrm{CPB}$ is followed by reduced postoperative blood loss and homologous blood requirement.

institute for Anesthesiology, German Heart Center, and the University Clinic (LMU), 80335 Munich, Germany

27

EFFECT OF TWO DIFFERENT DOSAGES OF APROTININ ON HEMOSTASIS AND GRAFTFUNCTION IN ORTHOTOPIC LIVER TRANSPLANTATION (OLT)

G. Himmelreich, W.O. Bechstein, M. Jochum, H. Gerlach, P. Neuhaus, and H. Riess

Aprotinin is believed to reduce transfusion requirements in OLT, but the optimal dosing of aprotinin is open to discussion

In an open, randomized studies 23 patients were randomly allocated to a bolus regimen of $3 \times 0.5$ Mio KIU aprotinin (B-group) and 10 to a continues aprotinin infusion with 200000 to $400000 \mathrm{KIU} / \mathrm{h}$ (Igroup). and transfusion rate as well as several parameters of hemostasis in plasma were investigated.

Results:

1. Aprotinin concentrations were consistently higher in the I-group

2 . In spite of longer operation times andstorage times in the I-group cathepsin B (released by hepatocytes) was higher in the B-group.

3. Thrombin-antithrombin complexes and elastase-proteinaseinhibitor complexes were higher in the I-group

4. There were no difference in AT III, protein C, C1-inhibitor or plasmin-antiplasmin complexes

$\underline{5}$. Concerning thrombelastography and plasma levels of tissue-type plasminogen activator there was less hyperfibrinolysis in the I-group.

6. There was a trend towards reduced perioperative transfusion requirement in the I-grou.

7. There were no thrombotic episodes perioperatively.

Conclusion:

Aprotinin used in OLT without concomitant heparin is safe and may be escalated resulting in reduced hyperfibrinolysis and hepatocellular protection. A large randomized study to determine the impact of aprotinin dosage on transfusion requirement is warranted.

Universitätsklinikum Rudolf Virchow, Department of Internal Medicine, Spandauer Damm 130, 14050 Berlin
28

BLOOD COMPATIBLE BIOMATERIALS THROUGH RESORBABLE ANTICOAGULANT DRUGS WITH COATINGS

A. Stemberger, E. Alt, G. Schmidmaier, J. Kohn*, G. Blümel

Implanted catheters, electrodes or stents adsorb plasma proteins and activate henostasis. Techniques have been developed to incorporate anticoagulants or affix them to plastic by chemical means, however, neither these procedures nor systemic anticoagulation eliminate the problems. The study group investigated rendering the surface of biomaterials thrombo-resistant by coatings using synthetic polymers of lactic-, glycolic acid and tyrosine derived polyarylates in combination with anticoagulants. The ex vivo and in vivo data, supported by scanning electronmicroscopy, reveal that uncoated biomaterials such as carbonfiber electrodes are covered within minutes by a coagulation plug rich in fibrin and platelets. Degradible coatings without anticoagulat reduce to some extent adherence of the fibrin rich coagulation proteins, coatings containing hirudin and prostacyclin inhibitors prevent the surface free from thrombi. Investigations of coagulation markers such as TATcomplexes and prothrombin fragments $\left(\mathrm{F}_{1}-2\right)$ corroborated these findings. These coating technique with the permanent disintigration of polymers and slow release of anticoagulants prevents thrombus formation from biomaterials.

*Present adress: Department of Chemistry

Rutgers University-Busch Campus

Piscataway, NJ 08855-0039, USA

\section{9}

REDUCTION OF THE CONTACT ACTIVATION SYSTEM BY BIOACTIVE ARTIFICIAL SURFACES IN EXTRACORPOREAL CIRCULATION

H.P. Wendel, W. Heller

Over the past decade our group has shown that the contact system of blood is activated in cardiopulmonary bypass (CPB), that heparins enhance this activation and that aprotinin reduces both this activation and blood loss in CPB. We developed an in vitro CPB model in order to assess the effects of added components to blood and new components in the artificial devices of CPB.

In the present study we compared membrane oxygenators with or without heparincoated surfaces under identical conditions in the CPB model. In the model recalcified $A C D$ blood was circulated in a closed system for 90 minutes at $28^{\circ} \mathrm{C}$. Blood samples were taken at various times during circulation. Heparin leveis were measured in the plasma samples together with various contact system components. Hemolysis, platelet count, platelet factor 4 and $\alpha_{1}$-proteinase inhibitor-PMN elastase complexes, were also determined.

No heparin could be detected during the whole period of recirculation in the samples from the coated oxygenators, showing the excellent adhesive quality of the hepatin coating. Analogous to a significantly greater fall in the platelet count with the noncoated oxygenators compared with the coated oxygenators (final mean counts of $17000 / \mu \mathrm{l} \pm 5000$ and $97000 / \mu 1 \pm 34200$ respectively after 90 minutes circulation) platelet factor 4 levels were significantly higher $(682.9 \% \pm 187.3$ and $95.8 \% \pm 46.5$ of the initial value respectively). Also hemolysis $(163.0 \% \pm 33.5$ and $125.1 \% \pm 23.0$ ) and degranulation of granulocytes as determined by PMN-elastase- $\alpha_{1}$-proteinase inhibitor complexes $(9349 \% \neq 662.5$ and $245.1 \% \neq 60.5)$ were significantly higher with the non-coated oxygenators. Contact system activation measured by increased kallikreinlike activity was also reduced in the heparin-coated system $(141 \% \pm 27.1$ and $108.7 \%$ = 18.4), Factor XII levels $(100 \%$ to $77 \% \pm 22.1$ and $100 \%$ to $88 \% \pm 16.7)$ fell much more markedly with the non-coated oxygenators.

The simultaneous reduction in contact system activation and effects on corpuscular blood components suggest that the use of heparin-coated oxygenators together with a lower beparin level in CPB could have clinical benefits in CPB-related postperfusion syndromes including hyperfibrinolysis and blood loss.

* Present address: Dept. of Thoracic and Cardiovascular Surg., Univ. of Tusbingen,

Calwerstr. 7, D-72076 Tuebingen, Germany 
HAEMOCOMPATIBILITY TESTING OF POLYMERS

U.T. Seyfert, S. Perkins, M. Kuemmel and E. Wenzel

Haemoincompatibility induced by contact with artificial materials is a major obstacle to further development of artificial organs and accounts for much of morbidity in circumstances when the blood is circulated through an extracorporeal system or a catheter is placed.

Materials and methods: 20 different polymers were provided as granula or catheter (Bayer Krefeld,FRG). Materials' s testing was performed using a Homburg centrifugation model and a chandler system. Different rheological shear stress conditions were examined. Citrated and hirudinized whole blood of healthy donors were used. We focused our attention on measurements of contact activation, platelet activation, thrombin generation, Fibrinogen - Fibrin conversion, haemolysis and proteolysis and developed a score for discriminating the different polymers.

Results: Elevated score points $(35 / 70)$ using HD/LDpolyethylene, ionomers $(20-25 / 70)$, acrylacidcopolymers (15-20/70), compared to styrols $(15 / 70)$ indicate haemoincompatibility. Polysulfone polymers demonstrate good haemocompatibility (score points 4-6). Polyvinylchloride polymers show a discrepant distribution of haemoincompatibility depending on flow characteristics.

Conclusions: various models are available for characterizing haemocompatibility of polymers. Flow characteristics and preanalytical effects (e.g. anticoagulants) may not be neglected. Our data suggest that it is possible to characterize the ex vivo blood - foreign surface interactions.

Address: Klinische Haemostaseologie Haus 75, Unikliniken, D-66421 Homburg

\section{Platelets III}

\section{1}

Epitope-Specific Flow Cytometric Analysis of Anti-Platelet Antibodies with Fluorescence-Resonance-Energy-Transfer (FRET)

G. Rothe', M. Koksch', V. Kiefel ${ }^{1}$, G. Schmitz

1 Institute for Clinical Chemistry, Central Laboratory, University of Regensburg,

F.R.G. ${ }^{2}$ Institute for Clinical Immunology and Transfusion Medicine, Justus-LiebigUniversity Gießen, F.R.G

Preformed antibodies against HLA-specific antigens, antibodies against platelet specific antigens, and circulating immunocomplexes may lead to an accelerated elimination of platelets after repeated platelet transfusion. Therefore, a pretherapeutic characterization of anti-platelet antibodies is of interest for the selection of blood donors.

Flow cytometry is a simple and sensitive tool for the quantification of surface bound immunoglobulin on donor-platelets after incubation with patient serum. A differentiation between antibodies against HLA-specific antigens, platelet-specific antigens, and circulating immune complexes, which bind to to the platelet Fc- $\gamma$-receptor-II (CD32), however, was not feasible with this method.

The goal of our study was the development of a flow cytometric assay for the epitope-specific analysis of human anti-platelet antibodies using fluorescence-resonance-energy-transfer. In a first step, donor platelets were incubated with patient serum and cell-bound human antibodies were quantified using polyclonal R-phycoerythrin-conjugated (excitation 488 $\mathrm{nm} / \mathrm{emission} 585 \mathrm{~nm}$ ) polyclonal antibodies. In a second step, plateletspecific glycoproteins, MHC-l-structures, and the Fc- $\gamma$-receptor-ll were stained with Cyan-5-labelled (excitation $585 \mathrm{~nm} / \mathrm{emission} 670 \mathrm{~nm}$ ) accep tor antibodies against CD29, CD41 $a, C D 42 b, \beta_{2}$-microglobulin and CD32. Upon monochromatic fluorescence excitation with a 488-nm argon laser the efficiency of light transfer from R-phycoerythrin to Cyan-5 is a direct measure of the distance of the human antibody which is being analyzed to the known epitope detected by the monoclonal antibody. The assay allows automatization and should be useful for the serial study of the quantity and specificity of anti-platelet antibodies. This should lead to more simple selection criteria for platelet donors in the handling of polytransfused patients.
32

HEPARIN-ASSOCIATED THROMBOCYTOPENIA DURING EXTRACORPOREAL LUNG ASSIST: SUCCESSFUL THERAPY WITH LOW WEIGHT HEPARINOID ORG 10172 DESPITE USING A COVALENTLY HEPARIN-BONDED BYPASS-CIRCUIT

W.Manert", H.Forst ${ }^{+}$, A. Niedermeier", Th. Hummel ${ }^{+}$, A. Greinacher ${ }^{*}$ and K.Peter

Heparin-associated thrombocytopenia (HAT) is a common, potentially serious side effect of heparin therapy, which can cause a therapeutic dilemma for the physician. We describe an unusual case of HAT in a patient with adult respiratory distress syndrome (ARDS) during extracorporeal lung assist (ECLA) with a covalently heparin-coated bypass-circuit. In the 28 years old man gas exchange almost completely was provided by a veno-venous bypass-circuit including two microporous membrane lungs. Moderate systemic anticoagulation (PTT $35-40 \mathrm{~s})$ was provided by intravenous infusion of low dose (500 - 900 $\mathrm{U} / \mathrm{h}$ ) unfractioned porcine heparin (Heparin-Na-25000-Ratiopharm ${ }^{\mathbf{R}}$, Fa. Ratiopharm, Germany) since admittance to the hospital. 27 days after the first application of intravenous heparin and nine days after beginning of ECLA platelet count began to decline from $204 \mathrm{G} / \mathrm{l}$ reaching a minimum of $58 \mathrm{G} / \mathrm{l}$. Diagnosis of HAT was revealed by heparin induced platelet activation (HIPA) assay. HIPA showed cross reactivity to various low molecular weight heparins, but not to the heparinoid Org 10172 (Orgaran ${ }^{R}$, Fa. Organon, Netherlands), which consequently was used for further intravenous anticoagulation. Org 10172 in a mean dose of $100 \mathrm{U} / \mathrm{h}$ resulted in anti-Xa-levels of $0,3-0,5 \mathrm{U} / \mathrm{ml}$. Despite persisting heparin-coated bypass thrombocytes rose to normal levels (159 G/1) within six days. To our knowledge this is the first patient with proven HAT, who was exposed to a covalently heparin-coated extracorporeal bypass. In contrast to ionically bonded intravasal catheters, which are a well-known cause of HAT, the covalently heparin-coated foreign surface did not support thrombocytopenia indicating that there is no leaching of heparin. An alternative explanation is that Org 10172 inhibited the pathomechanism of HAT as it has been shown in vitro. We conclude, that patients with HAT needing long term bypass such as ECLA, successfully can be managed by using a covalently heparincoated bypass circuit, supplemented by systemic anticoagulation with Org 10172 , when cross reactivity is excluded.

-Department of Anaesthesiology, Ludwig-Maximilians-University München, Klinikum Großhadern, Marchioninistraße 15, 81366 München, Germany Department for Clinical Immunology and Transfusion Medicine, Justus Liebig University Giessen, Germany 


\section{LABORATORY DIAGNOSIS OF HEPARIN-ASSOCIATED} THROMBOCYTOPENIA: ASSESSMENT OF DIFFERENT ASSAYS AND IN VITRO CROSSREACTIVITY STUDIES.

\section{A. Greinacher ${ }^{1}$, V.Dummel ${ }^{1}$, J.Amiral ${ }^{2}$, and C. Mueller-Eckhardt ${ }^{1}$}

As clinical diagnosis of heparin-associated thrombocytopenia (HAT) is often difficult, confirmation by sensitive laboratory assays is desirable. We prospectively compared the sensitivity of heparin induced platelet activation (HIPA) test and platelet aggregation test (PAT) using sera of 209 patients with the tentative diagnosis of HAT. Both assays were performed concomitantly with platelets of the same 4 donors who varied from day to day. In addition, all sera were assessed with a newly developed PF4/heparin ELISA.

Positive results were obtained with $33 \%$ of sera in the PF4/heparin ELISA, with $33.5 \%$ of sera in the HIPA test, and with $11.5 \%$ of sera in the PAT. PF4/heparin ELISA and HIPA test showed no difference in sensitivity ( $p=$ 0.27 MCNemar's test) and were more sensitive than PAT $\left(p<10^{-8}\right.$ McNemar's test). However, they recognized different patient cohorts. 9 indeterminate and 12 negative sera in the HIPA test were positive in the PF4/heparin ELISA. 8 of the 9 indeterminate sera caused platelet activation with high heparin concentrations in the HIPA test. 11 of the 12 negative sera contained no lgG but IgM (9) or IgA (2) HAT antibodies. Four indeterminate and 18 negative sera in the PF4/heparin ELISA were positive in the HIPA test. None of the sera positive in the PAT was missed in the HIPA but two of them were negative in the PF4/heparin ELISA. All sera were assessed with 4 LMWH and a LMW heparinoid (Org 10172) in the HIPA test. LMWH caused platelet activation with positive sera in $99 \%$, the LMW heparinoid in $10-20 \%$.

We conclude that HIPA test and PF4/ELISA are sensitive for diagnosing HAT and complement one another. The majority of HAT antibodies reacts with a PF4/heparin complex but in approximately $20 \%$ another protein ist involved. For crossreactivity studies HIPA test is a useful tool. This has yet not been proofen for the PF4/heparin ELISA.

1 Institut für Klinische Immunologie und Transfusionsmedizin, JustusLiebig Universität Giessen, Langhansstraße 7, 35385 Giessen. 2 Serbio Forschungs-Laboratorien, Gennevilliers, Frankreich.

\section{4}

Heparin-associated thrombocytopenia: isolation of the antibody and characterization of a multimolecular PF4-heparin complex as the major antigen.

A. Greinacher ${ }^{1}$, B. Pötzsch ${ }^{2}$, J. Amiral ${ }^{3}$, V. Dummel ${ }^{1}$, A. Eichner ${ }^{1}$, and C. Mueller-Eckhardt ${ }^{1}$

Sera of 35 patients with heparin-associated thrombocytopenia (HAT), strongly reactive in the serotonin release assay (SRA), were assessed in a piatelet factor 4 (PF4)/heparin ELISA. Both tests correlated closely (Kappa $0.596 ; p=8.44 \times 10^{-5}$, but one positive serum in the SRA was negative in the PF4/heparin ELISA. We have isolated the HAT antibody by absorption and elution of HAT sera using endothelial cells (HUVEC). Eluates gave similar results as the sera in the PF4/heparin ELISA (Kappa $0.726 \mathrm{p}=3.04$ $\times 10^{-6}$ ), which also correlated very closely with the SRA (Kappa 0.806; $p=$ $\left.1.45 \times 10^{-7}\right)$. This demonstrates that HAT antibodies bind to the same epitope on platelets and on endothelial cells. High heparin concentrations released PF4 in a dose dependent manner from microtiter plates if PF4/heparin, but not if PF4 alone, was covalently linked. Concomitant to the release of $\mathrm{PF} 4$, binding of HAT antibodies to PF4/heparin decreased, as indicated by median optical density (OD) values of $O D 0.947$ in the presence of buffer to $O D 0.181$ in the presence of $100 \mathrm{IJ} / \mathrm{ml}$ heparin. The latter values were similar to those when plates were coated with PF4 alone (median $O D$ 0.203). Binding of three eluates was not inhibited by high heparin concentrations and they reacted also with PF4 alone. We conclude that multimolecular PF4/heparin complexes represent the major antigen in HAT, which are present on platelets and endothelial cells. This multimolecular complexes present several antigens and form immune complexes after HAT antibody binding which activates platelets via the FCRll. Concommitant alteration of platelets and endothelial cells is a very likely explanation for the thromboembolic complications observed in HAT patients. In a few cases, PF4 alone can be recognized by the antibody. There is also evidence that other molecules might be involved in some patients.

1. Institut für Klinische Immunologie und Transfusionsmedizin, JustusLiebig Universität, Giessen. 2. Abteilung für Hämostaseologie und Transfusionsmedizin, Kerckhoff-Klinik, Max-Planck-Instiłut, Bad Nauheim. 3. Serbio, Forshungs-Laboratorien, Gennevilliers, Frankreich.
NCREASED THROMBOXANE PRODUCTION DURING PASSIVE SMOKING

H.Sinzinger, H.Kritz, G.Karanikas, Y.Efthimian, B.A.Peskar

Passive cigarette smoking (PS) has been identified as cardiovascular risk factor. As active smoking increases thromboxane (TX) production, we wondered, whether PS does as well. 12 smokers and 12 non-smokers were exposed in a $18 \mathrm{~m}^{3}$ room to the smoke of 30 cigarettes for 60 minutes. This exposure was repeated daily for 2 weeks. Blood for the determination of $\mathrm{MDA}$, serum and plasma $\mathrm{TXB}_{2}, 11$-dehydro $\mathrm{TXB}_{2}$ and the conversion of ${ }^{14} \mathrm{C}$-arachidonic acid to $\mathrm{TXB}_{2}$ and $\mathrm{HHT}$, respectively was drawn before, at the end and 6 hours after the exposure.

Acute exposure to PS causes an inmmediate significant increase of the parameters (except serum $\mathrm{TXB}_{2}$ ) being more pronounced in non-smokers as compared to smokers. While in smokers baseline values are reached again after 6 bours, non-smokers do not show a complete return of the parameters examined to baseline. Within a period of 2 weeks platelets of nen-smokers shen an idemical betravior to PS as those of smokers do.

\begin{tabular}{|c|c|c|c|c|}
\hline & & before & at the end & $6 \mathrm{~h}$ after \\
\hline MDA & non-smoker & $3,50 \pm 0,3$ & $4,20 \pm 0,4$ & $\begin{array}{c}3,66 \pm 0,2 \\
3,87+0,05\end{array}$ \\
\hline \multirow{2}{*}{$\mathrm{s}-\mathrm{IXBB}_{2}$} & smoker & $3,86 \pm 0,06$ & $\begin{array}{r}4,34 \pm 0,1 \\
215,9+2,7\end{array}$ & $\begin{array}{l}3,87 \pm 0,05 \\
215,8 \pm 1,2\end{array}$ \\
\hline & $\begin{array}{l}\text { thon-sinoket } \\
\text { smoker }\end{array}$ & $\begin{array}{l}218,3 \pm 3,4 \\
220,9 \pm 1,9\end{array}$ & $\begin{array}{l}210,9 \pm 2,7 \\
220,9+1,5\end{array}$ & $219,6 \pm 1,8$ \\
\hline \multirow[t]{2}{*}{$\mathrm{p}-\mathrm{TXB}_{\text {? }}$} & non-smoker & $2,16 \pm 0,2$ & $3,30 \pm 0,2$ & $2,33 \pm 0,2$ \\
\hline & smoke & $2,93 \pm 0,07$ & $3,83 \pm 0,08$ & $3,01 \pm 0,06$ \\
\hline \multirow[t]{2}{*}{ 11-DH TXB? } & non-smoker & $26,68 \pm 1,4$ & $30,26 \pm 2,0$ & $27,21 \pm 0,9$ \\
\hline & smoker & $31.31 \pm 0,6$ & $33,38 \pm 0,4$ & $30.11 \pm 2$ \\
\hline
\end{tabular}

These findings of an increased TX-production by platelets upon PS may offer a pathophysiological explanation for imbalanced haemostasis and the development of arterial lesions.

*Present address: Wilhelm Auerswald Atherosclerosis Research Group (ASF) Vienna, Nadlergasse 1, A-1090 Vienna, Austria.

\section{6}

BLOCKADE OF FIBRINOGEN - RECEPTOR AND BLEEDING RISK

C.M. Kirchmaier, A. Schirmer, D. Westrup, K. Becker-Hagendorff, B. Jablonka*, M. Meyer"*, M. Just*, H.K. Breddin

Glanzmann's thrombasthenia is a well defined genetic defect of platelet function caused by a total or partial deficiency of the glycoprotein IIb-Ilia complex (GP IIb-IIIa) on the platelet membrane.

The bleeding tendency in patients suffering from Glanzmann's thrombasthenia is discussed controversely. There are no concise reports on the bleeding risk and the amount of GP llb-llia as intact fibrinogen receptors an the platelet membrane. Also the extent of bleeding complications in patients ireated with a fibrinogen receptor antagonist is unknown. In order to get information on the predictive value of various laboratory parameters for bleeding, we investigated 35 patients with homozygous and heterozygous forms and two variants of Glanzmann's thrombasthenia.

In these patients we compared the bleeding history (a mild bleeding tendency it is bleeding postsurgery - it it life threatening bleeding requiring blood transfusions) with the skin bleeding time (simplate $I R$ ), ADP-induced platelel aggregation and ${ }^{125}$-fibrinogen binding on gelfitered platelets (GFP), platelet adhesion on siliconized glass, clot retraction, and GP Ilb-lila content detemined in an electro-immuno-assay (EIA). If necessary, GP Ilb-llla was further analyzed by twodimensional electrophoretic techniques.

Clot retraction had no predictive value for bleeding nor did it correlate with any of the parameters tested.

With the exception of a variant of Glanzmann's thrombasthenia, the number of fibrinogen receptors calculated from binding studies and EIA were comparable. The platelet GP lib-lila content of the two sibtings was reduced to $50 \%$ in the EIA but to 7 and $10 \%$ in the binding assay. The bleeding times were prolonged to 20 min.

ADP.induced aggregation in GFP was nomal if more then $50 \%$ of fibrinogen receptors were present on the platelet membrane.

Reduction of adhesion, platelet aggregation and number of fibrinogen receptors (binding assay) of more than $78 \%$ yealded a marked prolongation of the bleeding time. Only in these patients, bleeding events occured. Four major bleedings required blood transfusions. However, the severity of bleeding events was not correlated to the bleeding time measured.

We expect that a reduction of intact fibrinogen receptors of more than $78 \%$ caused by Thrombasthenia or receptor blockade may cause a bleeding risk.

Center of Internal Med., Dept. of Angiology, J.W.G.-University, Th. Stern Kai 7 , 60590 Frankfurt/M. and ${ }^{\star} \mathrm{C}$ asselia $A G$, Frankfurt/M., "*Med. Academy, Erfurt 
PLATELET HYPERREACTIVITY - MEASURED VIA THE STAGNATION POINT FLOW ADHESIO-AGGREGOMETER (SPAA) - AS AN INDICATOR OF PERIPHERAL ARTERIAL. DISEASE (PAD)

\author{
C.B. Reininger ${ }^{1}$, A.J. Reininger ${ }^{2}, M$ Spannagl ${ }^{3}$, B. Steckmeier',
L. Schweiberer" ${ }^{1}$,W. Schramm ${ }^{3}$
}

Plasma fibrinogen has been described as a major risk factor for peripheral arterial disease (PAD). Enhanced platelet activity has also been postulated in PAD patients. Direct, consistant verification of the previous has been provided with the SPAA. To evaluate the descriminatory power of this novel method, a larger collective was examined and test results compared to plasma fibrinogen concentration (PF) and to that of two indicators of ongoing coagulatory activity: thrombin-antithrombin-complex (TAT) and fibrin momomer (FM).

The SPAA provides on-line registration of platelet microthrombus formation upon a standardized surface superfused with platelet rich plasma (PRP). Mathematical evaluation yields constants reflecting both platelet adhesivity $(\mathrm{KpW}=\%)$ and aggregability $(\mathrm{Kpp}=\%)$. 45 healthy voiunteers (nonsmokers) and 51 nondiabetic PAD patients were examined. All patients received aspirin. Comparison of the following was performed: means, coefficient of variance (CV) sensitivity and specificity for manifest PAD.

The following results were obtained for the control group: $\mathrm{Kpw}=0.61 \% \pm$ $0.17 \%(\mathrm{CV}=11 \%), \mathrm{Kpp}=0, \mathrm{PF}=2.97 \mathrm{~g} / \mathrm{l} \pm 0.80 \mathrm{~g} / \mathrm{l}(\mathrm{CV}=19 \%)$ TAT $=$ $4.00 \mathrm{ng} / \mathrm{l} \pm 3.90 \mathrm{ng} / \mathrm{C}(\mathrm{CV}=64 \%), \mathrm{FM}=1.67 \mathrm{ng} / \pm 1.99 \mathrm{ng} / \mathrm{l}(\mathrm{CV}=50 \%)$. The patients yielded the following results: $\mathrm{Kpw}=1.35 \% \pm 0.23 \%, \mathrm{Kpp}=1.24 \% \pm$ $1.50 \%, \mathrm{PF}=3.94 \mathrm{~g} / 1+1.22 \mathrm{~g} / \mathrm{l}, \mathrm{TAT}=7.67 \mathrm{ng} / 1+10.10 \mathrm{ng} / \mathrm{FM}=4.59 \mathrm{ng} / \mathrm{I}$ 5.52ngl. Respective sensitivity and specificity: Kpw (98\%, 96\%), Kpp $(100 \%, 100 \%)$, PF $(40 \%, 86 \%)$, TAT $(30 \%, 72 \%)$ and FM $(58 \%, 76 \%)$. Significant differences in group means were observed for: Kpw/Kpp (p<0.001), $P F(p<0.01)$ and FM $(p<0.05)$.

When compared to the other 3 parameters, platelet function measured with the SPAA demonstrated the least intraindividual variance and greatest predictive power with respect to PAD. These resulis indicate the SPAA to be a very sensitive platelet function test suitable for monitoring the effectiveness of antiplatelet therapy.

1 Chirurg. Klinik Innenstadt, Ludwig-Maximilians-Universität, NuBbaumstr. 20 80336 München, 2 Anatomisches Institut, Techn. Universität München, 3 Klinikum Innenstadt, Ludwig-Maximilians-Universität München

\title{
Malignancy and Haemotasis
}

38

Tumor-Associated Proteolysis: Prospects in Clinical Decision Making and Tumor-Biology oriented Therapy.

Henner Graeff, Olaf Wilhelm, Fritz Jănicke and Manfred Schmitt, Frauenklinik der Technischen Universität, Ismaninger Str. 22, 81675 München

The malignancy of solid tumors is related to their proliferation rate and to their capacity for invasion and metastasis; these two mechanisms of tumor spread may eventually cause the death of the tumor patient. The potential of cancer cells to invade the surrounding tissue and to metastasize is correlated with the formation and degradation of structural elements in the vicinity of the tumor cells. This enables tumor cells to disintegrate the extracellular matrix (tumor stroma) and the basement membranes. Since the structure of the penetrated tissue consists mainly of proteins, e.g. fibronectin, fibrin, proteoglycans or collagen, the primary substances used by a tumor for invasion and metastasis are proteases.

There is increasing evidence that the urokinase-type plasminogen activator (UPA) and its inhibitor PAI-1 play a key role in tumor-associated proteolysis. This is reflected by the observation that the uPA and the PAI-1 content of breast cancer tissue are closely related to relapse-free and overal survival. Tumor (and stroma) cells synthesize and secrete uPA. Upon binding to a high affinity receptor (UPA-R) and the cell surface uPA activates plasminogen to the active serine protease plasmin, which is also bound to the cell surface via a specific receptor. Tumor cells also produce PAI-1, enabling the formation of the extracellular matrix at the site of metastasis. Thus, the presence of both uPA and PAI-1 modulates the invasive and metastatic phenotype of cancer cells.

Pathophysiological findings about the mechanism of tumor-associated proteolysis and their role in invasion and metastasis implies new forms of biological therapy for solid tumors. The interaction of urokinase and the urokinase receptor on the tumor-cell surface can be inhibited by uPA-R analogues (scavenger effect). Another therapeutic approach might be to inhibit uPA synthesis in tumor cells by the application of antisenseoligonucleotides suppressing the UPA expression. Both substances, the recombinant $\mathrm{UPA}-\mathrm{R}$ analogue and the oligonucleotide directed against the translation initiation site of UPA mRNA decreased the invasiveness of cancer cells in a model system.
ALTERATIONS OF HEMOSTASIS IN THE COURSE OF BONE MARROW TRANSPLANTATION

C. Salat, E. Holler, B. Reinhardt, HJ. Kolb, E. Hiller

Thrombocytopenia following radiochemotherapy is the most important cause of bleeding problems in the early phase after bone marrow transplantation (BMT).

Moreover, after the self-limited effects of the preparative regimen in the early phase the hemostatic system is involved in transplant related complications as graft versus host disease (GvHD), septicemia and veno-occlusive disease (VOD).

In GvHD endothelial cell damage is a crucial pathogenetic step. It can result in gastrointestinal bleeding or impairment of liver function with consecutive hypocoagulability.

Septicemia can lead to disseminated intravascular coagulation and enhance bleeding.

VOD, which is characterized by an occlusion of small intrahepatic venules, represents the most important thrombotic complication. It is assumed that endothelial cell injury caused by the conditioning regimen triggers the coagulation cascade and induces the deposition of coagulation factors in the affected venules. Whereas a reduction of protein $C(P C)$ in VOD patients was described by others our patients (four of 32) presented with normal PC levels and only low protein $S$ levels were noted in the first week after BMT. However, in all four patients with VOD an extreme elevation of PAI-1 without concomitant rise of tPA was detected in our study suggesting that hypofibrinolysis is involved in the pathogenesis of the disease. Regarding these results treatment with rtPA and prophylactic application of low dose heparin in spite of thrombocytopenia seem to be useful therapeutic approaches.

Med: Klinik III, Klinikum Grosshadern der LudwigMaximilians-Universität München 
COAGULATION STUDIES IN PATIENTS WITH APL DURING TREATMENT WITH ATRA, IN CASES OF RETINOIC ACID SYNDROME AND IN REMISSION

\section{P.Knöbl, S.Kapiotis, K.Laczika, U.Jäger, A.Kyrle, W.Speiser, K.Lechner}

Acute promyelocytic leukaemia (APL) is associated with disseminated intravascular coagulation (DIC), which can be accelerated by induction of cytostatic chemotherapy and causes a markedly risk for (intracerebral) haemorrhage. Complete remissions of APL can be achieved by differentiation-inducing therapy with all-trans retinoic acid (ATRA).

We studied hemostatic parameters (fibrinogen, coagulation factors, thrombin-AT III complex (TAT), D-dimer, prothrombin-fragment $\left.\left(\mathrm{F}_{1,2}\right)\right)$ in 7 patients with APL during induction therapy with ATRA $\left(45 \mathrm{mg} / \mathrm{m}^{2} / \mathrm{d}\right)$, in 4 patients developing a severe retinoic acid syndrome and in 9 patients in haematological $\mathrm{CR}$.

All patients had signs of coagulation activation before start of therapy (median fibrinogen $130 \mathrm{mg} / \mathrm{dl}$, D-dimer $2200 \mu \mathrm{g} / \mathrm{L}$, TAT $16.5 \mu \mathrm{g} / \mathrm{L}, \mathrm{F}_{\mathrm{L}, 2} 5 \mu \mathrm{g} / \mathrm{L}$ ), white blood cell count ranged between 0.6 and $45 \mathrm{G} / \mathrm{L}$, platelet count $22-83 \mathrm{G} / \mathrm{L}$. Median fibrinogen levels increased consecutively after initiation of ATRA (day 3: $124 \%$ of baseline, day 8 : $120 \%$ ), as well as the platelet count. Median levels of activation markers decreased significantly (TAT to $31 \%$ of baseline, D-dimer to $47 \%$ and $F_{1,2}$ to $64 \%$ ).

Four patients developed a retinoic acid syndrome with hyperleukocytosis (median 25 $\mathrm{G} / \mathrm{L}$ at day 14). One of these patients died from myocardial infarcion, 2 patients required mechanical ventilation and hemofiltration, but recovered completely. Activation markers increased significantly in these patients (TAT $207 \%$, D-dimer $347 \%$ and $F_{1,2} 404 \%$ ), but fibrinogen levels and platelet count remained unchanged. All patients achieved complete haematological remission. Molecularbiological analysis revealed CR in 3 patients (no PML-RAR $\alpha$ fusion product detectable by PCR). Platelet count, fibrinogen and levels of activation markers were normal in these patients. However, no clear cut relationship between molecularbiological detectable minimal residual disease and coagulation parameters could be found.

Our data show, that treatment with ATRA can reduce the coagulation disorder and the risk for severe bleeding in patients with APL. Whether the increase of activation markers in patients with retinoic acid syndrome is secondary to endothelial cell darnage or caused by expression of procoagulant factors by differentiating granulocytes remains to be established, as well as the possible importance of coagulation parameters in monitoring patients in $\mathrm{CR}$.

Dept.Med. 1, Div. Haematology and Haemostaseology, Univ. of Vienna Wăhringer Gürtel 18-20, A-1090 VIENNA, AUSTRIA

41

\section{TISSUE FACTOR PARTICIPATES IN METASTASIS BY TWO DIFFERENT PATHWAYS}

V. Borcea, Y. Deng, Y. Zhang, P. P. Nawroth

Recently we showed that stable transfection of $B-16$ melanoma cells with tissue factor (TF) regulates tumor growth and angiogenesis. Overexpression of TF induces VEGF, while suppressing the expression of thrombospondin (TSP).

When the metastatic potential of the stable transfectants was tested, we found increased numbers of lung metastases after i.v. injection of TF antisense transfectants (not expressing significant amounts of TF), when compared to wildtype, vector transfected or sense TF (overexpressing TF) transfected cells. Anticoagulation with warfarin reduced metastasis of TF expressing tumor cells, but not of antisense transfectants.

No difference was noted when the degradation of matrix proteins by the stable transfectants was tested. When nuclear run on experiments were performed, TSP was decreased by sense transfection and increased 25 fold in antisense transfectants. This implies, that $T F$ can participate in metastasis by two pathways :

1. TF enhances metastasis by a coagulation dependent increase in tumor cells expressing TF conly $T F$ expressing cells were reduced by warfarin).

2. Transfection of B-16 melanoma cells with TF antisense, reducing TF expression, enhances metastasis by a coagulation independent pathway, possibly involving expression of cell adhesion molecules.
STIMULATION OF TISSUE FACTOR EXPRESSION IN CANCER CELLS BY A MEDIATOR FROM LPS-STTMULATED MONONU. CLEAR BLOOD CELLS

T. Luther, M. Grosser, C. Flössel, H. Großmann, M. Kotzsch, and M. Müller

Tissue Factor (TF) is the primary cell-bound initiator of the coagulation protease cascade, finally leading to formation and deposition of fibrin. Different tissues, including epidermis, cerebral cortex, and epithelial layers, express TF constitutively. Until now, however, only little is known on regulatory mechanisms of that constitutive TF expression. Recent studies have shown TF protein to be expressed in human breast cancer tissues in highly distinct and hetreogeneous patterns, which could suggest that regulatory factors act locally in vivo.

This lead us to test whether different cytokines and growth factors are capable to induce TF expression in different breast cancer cell lines in vitro. Interestingly, in one of the cell lines tested (T47D), TF transcription could be upregulated only by a supernatant from LPS-stimulated mononuclear blood cells, but not by any of the cytokines and growth factors found to be effective with other breast cancer cell lines. Using T47D cells, we found that treatment with purified supernatant in a concentration dependent manner led to an enhancement of TF expression peaking after 8-12 hours. Production and/or secretion of the expected mediator from mononuclear blood cells can be blocked by cycloheximide. Initial biochemical characterization suggests that this mediator is an oligopeptide of about 1000 Dalton. Our results support the hypothesis that this mediator from mononuclear blood cells could be of importance for modulation of the "constitutive" TF expression of cancer cells.

Supported by Grant $0310018 \mathrm{~A}$ from BMFT.

Technical University of Dresden, Institute of Pathology, Fetscherstr. 74, 01307 Dresden, Germany 
Hirudin for Improvement of Thrombolysis (HIT Study): A Dose Escalating Study

Reiner von Essen, Karl-Ludwig Neuhaus, Walter Niederer, Ulrich Tebbe, Horst Nebelsieck, Michael Roth for the ALKK-Study Group, FRG

HBW 023 (recombinant hirudin) was investigated in a sequential design as an adjunctive therapy with front loaded rt-PA $(100 \mathrm{mg} / 90 \mathrm{~min})$ in patients suffering from acute myocardial infarction. The primary endpoint was a high early sufficient reperfusion rate (at 60 min $>65 \%$ TIMI 3 ), a reocclusion rate of $<10 \%$ within 48 hours and a low bleeding rate $(<10 \%)$.

Therapy was started with an i.v. bolus, followed by a continuous i.v. infusion over 48 hrs. Three dose groups were investigated in a sequential design. Initial boli were $0.1,0.2$ and $0.4 \mathrm{mg} \mathrm{HBW} 023 / \mathrm{kg} \mathrm{b.w.,} \mathrm{infusion}$ rates were $0.06,0.10,0.15 \mathrm{mg} / \mathrm{kg}$ b.w./hr. At $30,60,90$ minutes, $36-48$ hrs and on discharge coronary angiographies were obtained. In comparison to baseline, aPTT ratios ranged between 1.5 and 4.0 fold. TTMI 3 patency (percentage) were:

\begin{tabular}{|l|l|l|l|l|l|}
\hline Dose group & $\mathbf{n}$ & $\mathbf{3 0}$ min & $\mathbf{6 0}$ min & $\mathbf{9 0}$ min & $\mathbf{3 6 - 4 8}$ hrs \\
\hline I & 18 & 35.7 & 50.0 & 66.7 & 72.2 \\
\hline II & 42 & 43.8 & 57.1 & 71.4 & 75.6 \\
\hline III & 81 & 44.6 & 60.5 & 76.2 & 92.0 \\
\hline
\end{tabular}

Reinfarction occurred in 0 (Dose group I), 2 (II) and 1 patient (III). Reocclusions were found in $6.0 \%$ (Dose group I), $5.6 \%$ (II) and $1.2 \%$ (III). In total 3 patients had spontaneous haemorrhage (1 in Dose group I, 2 in II). Overall in-hospital mortality was $2.8 \%$.

Conclusion: HBW 023 in conjungtion with rt-PA showed a dosedependent increase in early, complete and sustained patency with a reduction in reocclusion rates. The risk of bleeding did not appear to increase with higher dosages. Further studies to investigate the benefit of HBW 023 in broader patient populations appear warranted.

\section{4}

Recombinant hirudin produces dose-dependent, stable and effective anticoagulation in patients with thrombolysis for acute myocardial infarction

U. Zeymer, M. Mateblowski, K-L. Neuhaus

Med. Klinik II, Staedtische Kliniken Kassel, Germany

Consistently effective anticoagulation is associated with a higher patency of the infarct related artery after rt-PA thrombolysis. In a dose finding study we used recombinant hirudin (HBW 023) as conjunctive therapy in patients with thrombolysis (front-loaded $\mathrm{rt}$ PA $100 \mathrm{mg} / 90 \mathrm{~min}$ ) for acute myocardial infarction (AMI). Hirudin therapy was initiated with a bolus of a) 0,1, b) 0,2 or c) $0,4 \mathrm{mg} / \mathrm{kg}$ bodyweight followed by an infusion of a) 0,06, b) 0,1 or c) 0,15 $\mathrm{mg} / \mathrm{kg} / \mathrm{h}$ over $48 \mathrm{~h}$. The timecourse of activated partial thromboplastin time (aPTT) and activated clotting time (ACT) in dose groups a) $(n=5), b)(n=8)$ and $c)(n=10)$ during the hirudintreatment is shown in the figure.

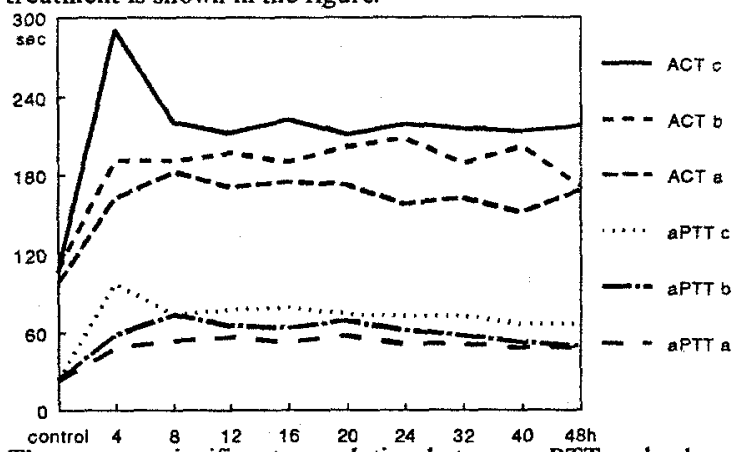

There was a significant correlation between aPTT and plasma level of free-hirudin $(r=0,62, p$-value $=0,002)$. During hirudin treatment we saw 1 reocclusion and 1 major bleeding. After $48 \mathrm{~h}$ all patients had a TIMI grade $2 / 3$ flow in the infarct related artery.

CONCLUSION: Recombinant hirudin as conjunctive therapy in patients with thrombolysis for AMI produces a dose-dependent and stable prolongation of aPTT and $A C T$, leading to an effective anticoagulation.
RECOMBINANT HIRUDIN AS A PERIPROCEDURAL ANTITHROMBOTIC IN CORONARY ANGIOPLASTY FOR UNSTABLE ANGINA PECTORIS G. Hafner, H.J. Rupprecht, M. Luz, W. Terres, A. Jessel, J. Meyer, and W. Prellwitz

Percutaneous transluminal coronary angioplasty (PTCA) is often complicated by thrombotic abrupt vessel closure in patients with unstable angina pectoris (UAP). The present multicentric trial was to determine the feasibility of two dose regimens of recombinant hirudin (r-hirudin; HBW 023) compared to standard heparin in patients undergoing PTCA for UAP.

At five participating centres, 61 patients were randomly enrolled in one of two sequential groups of r-hirudin (group I: $0.3 \mathrm{mg} / \mathrm{kg}$ i.v. bolus, $0.12 \mathrm{mg} / \mathrm{kg} / \mathrm{h}$ i.v. infusion; 21 patients; group II: $0.5 \mathrm{mg} / \mathrm{kg}$ i.v. bolus, $0.24 \mathrm{mg} / \mathrm{kg} / \mathrm{h}$ i.v. infusion; 19 patients) or in a heparin control group (150 IU/kg i.v. bolus, $20 \mathrm{IU} / \mathrm{kg} / \mathrm{h}$ i.v. infusion; 21 patients). Antithrombotic therapy was started directly pre-PTCA and continued for 24 hours. It was followed by a low dose anticoagulant infusion for another 24 hours (r-hirudin: $0.04 \mathrm{mg} / \mathrm{kg} / \mathrm{h}$; heparin: $7 \mathrm{IU} / \mathrm{kg} / \mathrm{h}$ ). Activated partial thromboplastin time (aPTT), activated clotting time (ACT), r-hirudin plasma concentrations by both immunological and functional assay, thrombinhirudin-complex (THC), thrombin-antithrombin III-complex (TAT), and prothrombin fragment $\mathbf{I}+2(\mathrm{~F} 1+2)$ were closely monitored.

In total, five major cardiac events were observed. In the r-hindin group I, one patient died, in the group II, one patient suffered acute myocardial infarction, and in the heparin group, one patient died and two patients developed an abrupt coronary occlusion. The aPTT prolongations versus baseline were found to be 2-fold and 2.5-fold in r-hirudin group I and II, respectively, and 3-4-fold in the heparin group. There was a good correlation between aPTT and ACT values in the $r$-hirudin groups when compared with the heparin group. The functional assay for the determination of $\mathrm{r}$-hirudin plasma concentrations was validated and showed excellent correlations to the immunological technique. A trend towards higher THC, TAT and $\mathrm{Fl}+2$ plasma concentrations was seen in the r-hirudin group 1 when compared with the r-hirudin group II and the heparin group, respectively.

The present study showed the feasibility of a periprocedural antithrombotic regimen with r-hirudin for patients undergoing PTCA for UAP.

Institute of Clinical Chemistry, University of Mainz, Langenbeckstr 1, D-55101 Mainz FRG

46

RECOMBINANT HIRUDIN AS AN ANTICOAGULANT IN OPEN-HEART SURGERY: A CASE REPORT

B. Pötzsch', S. Iversen', F. C. Riess', N. Tzanova ${ }^{3}$, C. Seelig', G. Nowak', and $\mathrm{G}$. Müller-Berghaus ${ }^{\top}$

The safety and efficacy of the thrombin inhibitor r-hirudin as an anticoagulant during open-heart surgery has been demonstrated in the pig model ( $F$. C. Riess, Throm. Haemostas. 69: 910, 1993, abstr.). So far, r-hirudin has not been used during open-heart surgery in patients. We report on a 29 year old man suffering from chronic pulmonary embolism, lupus anticoagulant, and heparin-induced thrombocytopenia. $r$-Hirudin (supplied by the Behringwerke, Marburg) was the only suitable anticoagulant to perform pulmonary thrombendarterectomy since the heparinoid Orgaran induced platelet aggregation similar to heparin. A bolus injection of $r$-hirudin of 0.75 $\mathrm{mg} / \mathrm{kg} \mathrm{b.w}$. was admistered $10 \mathrm{~min}$ before start of the extracorporeal circulation (ECC), and the heart-lung machine (HLM) was primed with $35 \mathrm{mg}$ $r$-hirudin. Ecarin clotting time and aPTT values were determined in intervals of $10 \mathrm{~min}$ for monitoring of $r$-hirudin dosis. Plasma levels of $r$-hirudin were kept constant between 4.0 and $5.0 \mu \mathrm{g} / \mathrm{ml}$ during the entire period of the HLM procedure for $267 \mathrm{~min}$. Ten min before end of ECC, plasma levels of r-hirudin were reduced to $2.0 \mu \mathrm{g} / \mathrm{m} /$ by hemofiltration. During and after operation, bleeding complications were not seen. Platelet function tests, such as ADP- and collagen-induced aggregation, showed levels of $45 \%$ and $35 \%$ even after an ECC time of 180 min suggesting a reduced risk of bleeding compared to heparin anticoagulation. The present report demonstrates that $r$-hirudin can successfully be used as an effective anticoagulant during ECC. Moreover, the data of platelet function suggest that r-hirudin may reduce the postoperative risk of bleeding and may therefore be a more suitable anticoagulant to be used during open-heart surgery.

1. Hemostasis Research Unit, Kerckhoff-Klinik, Sprudelhof 11, D-61231 Bad Nauheim, 2. Klinik für Herz-, Thorax- und Gefäßchirurgie and 3. Klinik für Anästhesiologie, Johannes-Johannes-Gutenberg-Universität, Langenbeckstr. 1, D-55131 Mainz, and 4. Research Unit for Pharmacological Haemostaseology, Max-Planck-Gesellschaft, D-07740 Jena. 
FIBRIN-TARGETED OR PLATELET-TARGETED RECOMBINANT HIRUDIN INHIBITS FIBRIN DEPOSITION ON EXPERIMENTAL CLOTS MORE EFFICIENTLY THAN UNTARGETED HIRUDIN

C. Bode, K. Peter, M. Freitag, P. Mewald, M. Hudelmayer, J. Ruef, M.S. Runge*

Although the indirect thrombin inhibitor heparin and the more potent, direct inhibitor hirudin are useful in preventing thrombosis, a substantial opportunity remains for improving thrombus selectivity and antithrombin efficacy in highly thrombogenic situations. To explore the effect of targeting to the surface of platelet-rich and fibrin-rich clots, we chemically coupled hirudin to the Fab' of an antibody directed to platelet receptor GP IIb/IIIa (conjugate HAP) or one directed to the amino terminus of the fibrin $\beta$ chain that becomes exposed only after thrombin cleaves fibrinopeptide B (conjugate HAF). HAF was 1900 fold more potent than hirudin ( $p<0,0001)$ in thrombin inhibition on immobilized fibrin. A hirudinantifibrin IgG conjugate was equipotent, indicating that univalent binding was sufficient for effective targeting. HAF was 18 fold more potent than hirudin $(p<0,003)$ in inhibiting fibrin deposition on the surface of a human plasma clot. In comparing hirudin with HAP, $0,05,0,1$, and 1 unit of hirudin allowed deposition of 2230,1460 , and $443 \mu \mathrm{g}$ of fibrin, respectively, on platelet-rich clots. HAP at $0,05,0,1$, and 1 unit was more effective, allowing deposition of only 398,507 , and $278 \mu \mathrm{g}$ of fibrin. As antithrombins, fibrin-targeted and platelet-targeted recombinant hirudin both outperform hirudin on the surface of experimental human blood clots.

Innere Medizin III, Universität Heidelberg, Bergheimerstr. 58, 69115 Heidelberg und *Emory University, Atlanta, USA

\section{Heparin I}

48

HEPARIN PROLONGS CIRCULATION TIME OF OXIDIZED LOW DENSTTY LIPOPROTEIN IN RATS

G.Stehle*, A.Wunder, H.Sinn, E.A.Friedrich, H.H.Schrenk, B.Neufeld, C.E.Dempfle, H A.Dresel, J.Harenberg, W.Maier-Borst, and D.L.Heene

There is evidence that $\mathrm{Ox}$-LDL plays an important role during the atherosclerotic process. The presence of $O X-L D L$ in atherosclerotic lesions has been demonstrated. After scavenger receptor mediated uptake OX-LDL is stored in macrophages that subsequently transform to foam cells. We now report on the in vivo interaction between unfractionated heparin and oxidized LDL. In this study five groups of five male Wistar rats received either LDL or OX-LDL tagged with a residualizing ${ }^{131}$ I-tyramine-deoxysorbitol (TDS) label by iv injection $(100 \mu \mathrm{Ci}, 100 \mu \mathrm{g})$. Group 1 received TDSLDL alone, group $22 \mathrm{mg}$ of heparin $2 \mathrm{~min}$ prior to TDS-LDL, group 3 received a preincubated mixture of TDS-LDL and heparin, group 4 TDS-OXLDL alone, and group 5 received $2 \mathrm{mg}$ of heparin $2 \mathrm{~min}$ prior to TDS-OXLDL. Sequential scintigraphy was carried out and blood samples were drawn over a period of $60 \mathrm{~min}$. The blood concentrations of the intact TDSLDL ranged between $75 \%$ and $85 \%$ of the injected dose for group $1-3$. No significant interaction was found for heparin and LDL. After application of radioactive OX-LDL only $26 \%$ of the material were measured in circulation after $5 \mathrm{~min}, 10 \%$ after $15 \mathrm{~min}$, and $2.7 \%$ after $60 \mathrm{~min}$. After preinjection of heparin 2 min prior to OX-LDL tracer $44 \%$ of the substance was found in blood after $5 \mathrm{~min}, 28 \%$ after $15 \mathrm{~min}$, and $7.3 \%$ after $60 \mathrm{~min}$ These results indicate that heparin might partially compete the scavenger receptor mediated uptake of OX-LDL in vivo. They are in accordance with our prior observation that heparins are predominately metabolized by macrophage scavenger receptors (G.Stehle et al. J. Clin. Invest. 90:21102116 , 1992; G.Stehle et al. J. Lab. Clin. Med. 122, Dec. 1993). This direct interaction between heparin and the atherosclerotic agent OX-LDL further supports the hypothesis of an antiatherosclerotic effect of heparin

${ }^{*} 1$. Dept. of Medicine, Faculty for Clinical Medicine Mannheim, University of Heidelberg D-68167 Mannheim, and Dept. 05, Deutsches Krebsforschungszentrum, Im Neuenheimer Feld 280, D-69120 Heidelberg.

Supported by grant Ste 647/1-1 from Deutsche Forschungsgemeinschaft.
49

EFFECTS OF SINGLE IV AND SC INJECTIONS OF A SUPERSULFATED LOW MOLECULAR WEIGHT HEPARIN ON COAGUULATION PARAMETERS AND ON PLATELET FUNCTION E. Glusa, J. Schenk * **, W. Barthel*, F. Markwardt, A. Butti**, P. Radziwon **" and H.K. Breddin ****

A newly developed superfulfated low molecular weight Heparin ISSH. IKETON Pharmaceutici, Milan) has been studied in a phase I trial. 6 healthy volunteers received single iv injections of 0.14 $\mathrm{mg} / \mathrm{kg}, 0,33 \mathrm{mg} / \mathrm{kg}$ and $0,66 \mathrm{mg} / \mathrm{kg}$, with intervals of one week. 6 other volunteers received $0,33 \mathrm{mg} / \mathrm{kg}, 0,66 \mathrm{mg} / \mathrm{kg}$ and 1,0 $\mathrm{mg} / \mathrm{kg}$ subcutaneously. Clotting variables and platelet function were studied before and $10^{\circ}, 20^{\circ}, 30^{\circ}, 60^{\circ}, 120^{\prime}$ and $240^{\circ}$ after were studied before and $10^{\circ}, 20^{\prime}, 30^{\circ}, 60^{\circ}, 120^{\prime}$ and $240^{\circ}$ after
iv injection, and $30^{\circ}, 1,2,3,4$ and 8 hours after sc injections. The iv application caused a marked, dose-dependent prolongation of aPTT and Heptest. At $0,66 \mathrm{mg} / \mathrm{kg}$ baseline values were reached after 4 hours. Thrombin time and platelet adhesion were not significantly affected. Platelet induced thrombin generation time (PITT) was prolonged after $0,33 \mathrm{mg} / \mathrm{kg}$ and $0.66 \mathrm{mg} / \mathrm{kg}$ iv up to 60 minutes. After sc application there was also a dose-dependent prolongation of aPTT and Heptest with a maximum after 60 minutes. At the highest dose of $1 \mathrm{mg} / \mathrm{kg}$ baseline values were measured after 8 hours. The maximum increase in PITT occurred after 2 hours. SSH was well tolerated, no changes in blood pressure and heart rate were observed. The bleeding time measured before and 4 hours after iv or SC application was not significantly changed. Preclinical results and the present studies indicate that SSH may Preclinical results and the present studies indicate that $\mathrm{SSH}$ may doses could be used for prevention of thrombosis in humans.

Institute of Pharmacology, Medical Academy Erfurt * Clinical Pharmacology Academy Erfurt

* * Iketon Pharmaceutici Milan.

* * Dept. of Haematology, Medical School, Bialystok, Poland

*** Division of Angiology, Department of Internal Medicine, J. W. Goethe-University Frankfurt am Main,

Deutscher Titel: Wirkung von intravenösen und subkutanen injektionen eines supersulfatierten niedermolekularen Heparins dut Gerinnungsparameter und Plättchenfunktion 
DOES LOW MOLECULAR WEIGHT HEPARIN (LMWH) CROSS THE PLACENTA?

E. Hiller, A. Mohr, K. Hiller, C. Salat, and E.R. Weissenbacher

High molecular weight heparin (HMWH) is the antithrombotic drug of choice in treatment and prophylaxis of thromboembolic disease of pregnancy, since it is generally believed that HMWH does not cross the placenta. Little is known of the placental transfer of LMWH which for obvious reasons would be advantageous as an antithrombotic drug. Therefore, in a dual open perfusion model of the placenta according to Schneider and Leichtweiss we examined the possible maternal-fetal transfer of two commercially available LMWH (Fragmin, Fraxiparin). LMWH in therapeutic and prophylactic concentrations was added to the perfusion medium which was then introduced via the spiral arteries on the maternal side into the intervillous spaces. Heparin concentrations in samples collected from maternal and fetal veins were determined by measurement of anti-Xa (chromogenic assay) at definite time intervals. In a total of 20 perfusion experiments the anti-Xa activity in the samples of the maternal side ranged from 0.2 to 1.0 units, whereas no anti-xa activity was detectable in any of the samples from the fetal side. Therefore, we believe that LMWH does not cross the placental barrier.

Medizinische Klinik III and Frauenklinik, Klinikum Grosshadern der Universität München, D - 81377 München
52

A RANDOMISED, MULTICENTRE DOUBLE BLIND STUDY INVESTIGATING THE EFFICACY AND SAFETY OF THE LOW MOLECULAR WEIGHT HEPARIN ENOXAPARIN VERSUS UNFRACTIONATED HEPARIN IN THE PREVENTION OF THROMBOEMBOLISM IN IMMOBLISED MEDICAL PATIENTS

E. Lechler*, W. Schramm, C.W. Flosbach, and THE PRIME - Study group

In numerous clinical trials the efficacy and safety of low molecular weight heparin [LMWH] in the prevention of thromboembolism in surgery have been shown. In contrast, few studies have been performed in internal medicine. The aim of the present study was to compare the incidence of thromboembolic events (deep vein thrombosis [DVT] and pulmonary embolism [PE]) under the prophylaxis with $40 \mathrm{mg}$ Enoxaparin [E] (Clexane) od versus $5,000 \mathrm{IU}$ calcium heparin [UFH] tid in immobilised medical patients over a period of 7 days. Patients at high risk for thromboembolism were recruited with at least one more risk factor besides immobilisation. All patients were screened for thrombosis at study entry by B-scan or duplex sonography and at the end of the study period with duplex sonography. Positive results had to be confirmed by phlebography. PE was verified with pulmonary perfusion scan, angiography or - in case of death, if permitted - by autopsy.

477 patients received $E$ and 482 patients UFH. In 442 patients of the $E$ group and 443 of the UFH group all required investigations were performed. 6 patients $(1.4 \%)$ in the UFH group and 1 patient $(0.2 \%)$ in the E group developed thromboembolic events

In each of the treatment groups bleeding events were documented in $13 \mathrm{pa}-$ tients $(2.7 \%)$. Major bleedings occurred in 2 patients $(0.4 \%)$ in the $\mathrm{E}$ group and in $7(1.5 \%)$ in the UFH group. There was no difference in the kind of adverse events, but there were fewer and less severe ones with $\mathrm{E}$. Liver enzymes were significantly more frequently elevated with UFH than with $E .7$ patients in the E group died and 11 in the UFH group, details will be presented. No decrease in platelet count due to UFH or $\mathbf{E}$ was documented. Conclusion Enoxaparin $40 \mathrm{mg}$ od is at least as effective as 5,000 IU UFH tid in the prevention of thromboembolic events in medical patients at high risk. In respect to bleeding Enoxaparin may be safer than UFH.

* Dept. of Medicine, University of Cologne, Josef-Stelzmann-Strasse 9 ,

D-50924 Köln: for THE PRIME study investigator group

\section{3}

SUCCESSFUL USE OF ORGARAN (ORG 10172) IN INTENSIVE CARE PATIENTS WITH HEPARIN-INDUCED THROMBOCYTOPENIA TYPE ॥

U. Burkhard-Meier, D. Söhngen, H.P. Schultheiss, A. Greinacher,

B. Schwartzkopff, M. Vogt, A. Heyll, W. Schneider

Heparin-induced thrombocytopenia (HIT) with thrombosis (type II) occurs in about $0.05 \%$ of all heparin-treated patients. The venous and arterial thromboses cause a mortality of about $30 \%$. Immediate discontinuation of heparin is mandatory. Most patients, however, need further effective anticoagulation.

We report on our experience with the low molecular weight (LMW) heparinoid Orgaran in intensive care patients with HIT type II. We treated 3 males and 2 females, whose age was between 52 to 74 years. Underlying diseases requiring effective anticoagulation were acute myocardial infarction, catheterinduced thrombosis, deep venous thrombosis and pulmonary embolism. All patients were treated in the intensive care unit when effective intravenous heparin treatment was started. On day 7 to 15 of therapy, patients developed a new thrombo-embolic event or progression of preexisting thrombosis. There was one arterial embolus in the left lower leg, one progression of deep venous thrombosis in the right leg, one progression of thrombosis of cervical and thoracic veins, one deterioration of pulmonary embolism, and one acute Leriche syndreme. The thrombocyte nadir was reached on d2y 7 to 17 of heparin treatment, paralleling the thrombo-embolic event; platelet counts ranged between 9.000 to $85.000 /$ l (median 34.000/ul). The diagnosis of HIT type II could rapidly be confirmed by the platelet aggregation test and the heparin induced platelet activation (HIPA) assay. Heparin was withdrawn and Orgaran $(2000 \mathrm{U}$ bolus injection, $400 \mathrm{U} / \mathrm{h} \times 4 \mathrm{~h}, 300 \mathrm{U} / \mathrm{h} \times 4 \mathrm{~h}, 200 \mathrm{U} / \mathrm{h}$ continous infusion) applicated, because this was the only LMW heparin without crossreactivity. Thrombocyte recovery was observed in all patients 1 to 4 days after heparin withdrawal, a normal platelet count was measured in 3 patients 2 to 5 days later. No severe bleeding complications occured. Two patients died, one of therapy-resistant heart failure on day 9 of Orgaran treatment with a thrombocyte recovery from 9.000 to $81.000 / \mathrm{ul}$, and one of septic-toxic shock 2 days after surgery for acute Leriche-Syndrome. The other three patients recovered without further thrombo-embolic events.

Conclusion: The use of the LMW heparinoid Orgaran seems to be an effective intravenous anticoagulation for intensive care patients with HIT type II.

Present adress: Department of hematology, oncology and clinical immunology, Heinrich-Heine University, Moorenstraße 5, 40225 Düsseldorf 
54

\section{PATHOLOGICAL VALUES FOR aPTT AND QUICK DURING CHILDHOOD \\ A.-M. Mingers, P.Zeitler-Zapf}

We retrospectively evaluated all our coagulation analysis that was done during the last two years concerning the values for aPTT and Quick. The question of our study was whether aPTT and Quick values outside the normal adult range have clinical relevance for children.

Patients: All children who received a coagulation analysis (age range: birth-18 years).

Results: More than 300 children showed pathologically prolonged aPTT values; 38 children had reduced Quick values. Pathological aPTT values were caused by age related prolongations of aPTT, newly diagnosed hemorrhage diathesis (often shortage of F.XII), non congenital v.W.D., inhibitors of Lupus.

An additional statistical evaluation of the last 5 years showed the following results: 64 children had a F.XII-shortage, 29 of these 64 children had a congenital shortage, 16 of the 29 children with congenital shortage had a combination with another congenital disturbance of coagulation, 35 children had a non congenital shortage of F.XII. Pathologically reduced Quick values were caused by DIC $(n=17)$, by massive hemorrhage during surgery of patients without history of coagulopathy $(n=6)$ and congenital shortage of coagulation factors $(\mathrm{n}=2)$.

Conciusions: Pathologically reduced Quick values and prolongations of aPTT can have different causes. Shortage of F.XII and congenital combined coagulopathies have a higher incidence than previously reported.

Universitätskinderklinik, Josef-Schneider-Str. 2, 97080 Würzburg, Germany

KOAGULATION PARAMETERS AFTER CHANGE IN VITAMIN-K PROPHYLAXIS IN NEWBORNS

R. Rossi, O. Albrecht, H. Pollmann and E. Harms

Following the publication of GOLDING (BMJ 305:341, 1992) on the association of parenteral Vitamin-K supplementation to newborns and childhood cancer, we changed dose and application mode of Vitamin-K prophylaxis: prematures of less than $2000 \mathrm{gr}$ of birth weight and newborns with major illnesses like septicemia or gastrointestinal malformation received a single dose of 0.2 (instead of 1) $\mathrm{mg}$ of Vitamin K parenterally, whereas healthy newborns above $2000 \mathrm{gr}$ received $1 \mathrm{mg}$ Vitamin $K$ on days 1,5 and by week 6 orally.

After these changes we observed two major bleeding complications related to Vitamim-K deficiency: one newborn not supplemented with Vitamin-K had gastrointestinal bleeding, a second patient experienced intracranial haemorrhage; diagnostic workup revealed $\alpha_{1}$-antitrypsin deficiency and an intracranial DandyWalker malformation.

This study was performed to investigate the sufficiency of this reduced Vitamin-K supplementation. We report on 118 coagulation analysis performed between days 5 and 40 in 17 patients after oral and 34 after parenteral Vitamin K. After parenteral Vitamin K, only 2 patients had Quick values of less than $60 \%$, lowest value observed was $49 \%$; for the whole group, mean Quick value was $92 \%$. All patients after oral Vitamin K had Quick values well above $60 \%$, mean was $96 \%$. Within the group of patients with parenteral Vitamin-K supplementation, Quick values were found to be related to the formula fed: mean Quick value after hydrolysate feeding was $86 \%$ with $2 / 15$ patients below $60 \%$, whereas after conventional milk formula there was no Quick value below $60 \%$, mean was $98 \%$. Cumulative Vitamin-K supplement given by these formula was $1833 \mu \mathrm{g}$ after hydrolysate formula and $5264 \mu \mathrm{g}$ after conventional milk formula. Comparison of these results to a previously reported study from this institution after parenteral supplementation of $1 \mathrm{mg}$ Vitamin-K (BOOS, Pediatr Hem Oncol 6:113, 1989) showed no differences in Quick values.

We conclude, that reduced Vitamin-K supplementation as outlined above is sufficient to induce normal coagulation in newborns.

\section{6}

TISSUE FACTOR PATHWAY INHIBITOR (TFPI) IN CHILDHOOD I. Wendisch*, G. Weissbach and I. Harenberg

The tissue factor pathway inhibitor (TFPI) was investigated in more than 340 infants and children. The amidolytic method according to SANDSET and ABILDGAARD with nonactivated factors VII and X was used. The residual factor Xa activity was measured by aid of the chromogenic substrate S-2222 (Kabi Stockholm). In 28 children parameters of the lipid metabolism (cholesterol, triglycerids) were detected. In most newborns the TFPI values were found below the adults' mean. Beyond the $6^{\text {th }}$ day of life in healthy infants values within the adults' standard range can be expected. In sick children a broad variation of the activities was found. In patients with tumors and leukemias predominantly high or elevated levels were detected. Only in two cases the activity was below the adulds mean. In patients with septicemia und purulent meningitis we observed only values above the adults' standard. In patients with other inflammatory processes the behaviour was not so uniform. We have registered the inhibitor activity in most cases in the upper region of the adults' norm. In some patients suffering from encephalitis normal values were found. In many sick children the inhibitor behaves like an acute phase reactant, for example in two patients with acute infectious hepatitis. But in other cases this property cannot be recognized. In children only a weak correlation between TFPI and cholesterol as well as LDL cholesterol exists. This is in contradiction to the findings by SANDSET et al. with a close connection to the cholesterol level in adults. In our cohort of children the variation range of the cholesterol values was not so extended.

* Medical Faculty of the Technical University, Department of Pediatrics, Fetscherstr. 74, 01307 Dresden, Germany

57

CLINICAL AND LABORATORY EVALUATIONS IN PATIENTS WITH VON WILLEBRAND DISEASE

B. Zieger, KB. Thomas, U. Jessat, A. Zehenter, AH. Sutor.

Von Willebrand's disease (vWD) is a bleeding disorder that is heterogeneous in its modalities of clinical and laboratory manifestations. During the last year we have examined patients $(n=15)$ from eight unrelated families with vWD in order to reevaluate the diagnosis after establishing new methods for diagnosis and classsification of $\mathrm{vWD}$. The aims of this project were the classification of the patients' vWD type and the comparison of clinical symptoms and laboratory results.

All patients were interviewed according to a standardized questionaire with respect to spontaneous bleeding and bleeding after trauma or surgery. The routine laboratory tests included the skin bleeding time, platelet count, FVIII:C, Ristocetin-Induced-Platelet-Aggregation, von Willebrand Factor (vWF) Antigen (Ag), vWF:Collagen Binding Activity (CBA) and vWF Multimer. Plasma and platelet vWF were examined.

vWD type I was diagnosed in 9 patients (Type Vicenza in 2 patients).

Patients with VWD type I showed mild bleeding symptoms (epistaxis, haematoma). In contrast to most of our type I patients one patient with vWD type I Vicenza had more severe bleeding symptoms. Six patients have vWD type II (IIB in 2 patients and IIC in 4 patients). Most of the patients with vWD type II had moderate to severe bleeding symptoms, such that in 2 cases therapy with vWF concentrate was needed. Classification of the VWD into types I and II was possible using the plasma vWF-CBA/Ag ratio. In most patients with severe bleeding symptoms the platelet vWF was below normal and the skin bleeding time prolonged illustrating the importance of the platelet vWF determination in addition to the plasma vWF in the classification of patients with vWD.

Universitäts-Kinderklinik, Mathildenstraße 1, D-79106 Freiburg.

University Children's Hospital Münster, Albert Schweitzer Str. 33, 48129 Münster 


\section{IS F XII-DEFICIENCY IN CHILDHOOD CORRELATED WITH INFECTIOUS DISEASE? \\ I. Martinex, E. Lenz, C. Escuriola, S. Becker, B. Zwinge, D. Klarmann,} W. Kreuz.

Hereditary homozygous F XII - deficiency is a rare coagulopathy. It is inherited in an autosomal recessive pattern. Until now poor information exists about the heterozygous form of F XII - deficiency.

Patients with severe deficiency of F XII tend to develop thrombotic complications; bleeding tendency is rare. Mild F XII - deficiency does not seem to be of any clinical significance (Lämmle et al., 1991).

As a cause for preoperatively detected aPTT - prolongation laboratory investigations may reveal F XII - deficiency.

From 1/1990 until 11/1993 we detected 40 cases of F XII-deficiency with residual activities ranging from $7 \%$ to $53 \%$ (normal range $>54 \%$ ).

In our patients' group mean age was 6 years $(1-13,8$ yrs).

In 25 cases out of 40 patients the reason for coagulation analysis was preoperative aPTT - prolongation. In 22 patients surgical procedure of the squamous tissues (exclusively tonsillectomies and adenotomies) was planned. 2 patients were scheduled for circumcision and 1 patient for herniotomia. 7 patients presented because of bleeding symptoms, 2 patients becanse of aPTT - prolongation and in 6 patients F XII - deficiency occured with an other coagulopathy.

In 11/40 patients symptoms of bleeding tendency such as nose or gingival bleeds were reported. In 7 out of these 11 patients with bleeding symptoms von-Willebrand-disease or other coagulopathies could be diagnosed.

Only $2 / 11$ patients with bleeding symptoms had isolated F XII-deficiency. In $9 / 40$ patients $F$ XII - deficiency was also detected in relatives indicating hereditary F XII - deficiency.

Infection could be proved in $25 / 40$ patients by means of clinical signs and laboratory investigations. Follow-up indicated a temporary F XII - deficiency.

Thus, we suppose F XII - deficiency being connected with infectious diseases in a large number of children.

Department of Pediatrics, University Hospital Frankfurt, TheodorStern-Kai 7, 60596 Frankfurt am Main.
59

ARE SOME PRESUMED CASES OF AFIBRINOGENAEMIA DYSFIBRINOGENAEMIAS?

I. Michelmann, S. Eckhoff-Donovan, W. Nürnberger, R. v. Kries and U. Göbel

The conventional definition of afibrinogenaemia is absence of clottable fibrinogen associated with undetectable fibrinogen antigen using standard technices (RID). Detectable normal or slightly subnormal fibrinogen concentrations and reduced function are suggestive of dystibrinogenaemia. With routine analyses (aPTT, PT, TCT and fibrinogen with the method of Clauss and RID), no detectable fibrinogen was found in two children of our hospital, but further proteinchemical analyses suggested that both cases were dystibrinogenaemias.

Case 1 is a now 6 months old turkish girl of a consanguine parents, in whom the diagnosis was made after prolonged puncture bleeding in the neonatal period. No spontaneous bleeding was ever observed.

Case 2 is a now 17 years old boy with a history of bleeding complications as intramuscular bleeding, hematoma and spontaneous bleeding in different joints.

Further analyses were carried out by separating reduced and non-reduced patients plasma on SDS-Polyacrylamide-gelelectrophoreses with following proteinestimation using coommassie- and silverstaining. In comparison to normal samples, a reduced HMW-like band, an intensified HMW'-like band and a reduced LMW-like band of the fibrinogens were found for both patients but there was no difference in the total amount in relation to normal plasma (estimated by intensity of staining). These results were confirmed by the carbohydrate-specific PAS-staining of the molecule.

The antigenity of the patients fibrinogens was proved with two different polyclonal antifibrinogen-antibodies by Western-blotting. A strictly lowered antigenity for non-reduced and reduced plasma samples was found. These observations show that the diagnosis of afibrinogenaemia only by functional and standard immunologic methods is not sufficient.

Heinrich-Heine-University, Dep. of Pediatric Hematology and Oncology, Moorenstr. 5, D-40225 Düsseldorf

Supported by the "Elterninitiative Kinderkrebsklinik e.V.", Düsseldorf.

\section{Molecular Biology}

60

\section{CHARACTERIZATION OF THE CAUSATIVE GENETIC DEFECTS IN TEN PATIENTS WITH HEMOPHILIA B \\ J.Walter, B.Krinninger, I.Pabinger-Fasching, H.H.Watzke}

Factor IX (FIX) is a vitamin K-dependent plasma protein essential for normal hemostasis. Lack of functional FIX results in the hereditary bleeding disorder hemophilia $\mathrm{B}$. We describe the molecular basis of hemophilia B in ten patients, who were investigated at our department. Characterization of the mutations was performed by amplification of all eight exons and exon-intron junctions by PCR and subsequent genomic sequencing of the products. We identified the following causative mutations: FIX Vienna 1: a deletion of nucleotides (nt) 20530-20532 in exon VI leading to the loss of the codon for Gly-184. FIX Vienna 2: a deletion of nt 6343-6362 in exon II resulting in a premature stop-codon at nt 6378-6380. FIX Vienna 3: a point-mutation at nt $17704(C>G)$ in exon $V$ resulting in the substitution of Gin-97 by Glu. FIX Vienna 4: a point-mutation at $n t 17761(C>T)$ in exon $V$ leading to a stop-codon at Aig-116. FIX Vienna 5: a point-mutation at nt $10415(\mathrm{C}>\mathrm{G})$ in exon IV resulting in the substintion of Pro-55 by Ala. FIX Vienna 6: a pointmutation at nt $6583(\mathrm{C}>\mathrm{T})$ in exon II altering Thr-38 to Ile. FIX Vienna 7 a point-mutation $(G>C)$ at nt 31276 in exon VIII leading to the substitution of Trp-385 by Cys. FIX Vienna 8: a deletion of nt 6700 in exon III resulting in a premature stop-codon at nt 10422-10424. F IX Vienna 9: a point mutation at $n t 10392(\mathrm{G}>\mathrm{T})$ in exon IV resulting in the substitution of Asp-47 by Val. FIX Vienna 3 was found in two appearantly unrelated patients. FIX Vienna 4 and 5 have been previously described, whereas FIX Vienna 1,2,3,6,7 and 8 are novel FIX-mutants.

Univ.Klinik für Innere Medizin I, Abteilung für Hämatologie und Hämostaseologie, Währinger Gürtel 18-20, A-1090 Wien

\section{1}

\section{FACTOR X"VIENNA": MOLECULAR ANALYSIS AND IN VITRO EXPRESSION OF A SEVERE CRM-NEGATIVE FX DEFICIENCY H.H.Watzke, K.Lechner, P.Larson, K.A.High}

Factor $\mathrm{X}(\mathrm{FX})$ is a vitamin $\mathrm{K}$-dependent plasma protein which plays a central role in blood coagulation. Cross reacting material (CRM) negative FX deficiency is characterized by a lack of detectable FX antigen in the plasma. Here we describe the molecular basis of a CRMnegative FX deficiency in a patient with a severe bleeding diathesis. The propositus is a 4 year old boy who suffered a cerebral hemorrhage within his first year of life. His PT and APTT are very prolonged. The FX activity level is $<1 \%$ and the FX antigen level is $<5 \%$ of normal. The coagulation times of his parents are normal. Their FX activity levels and FX antigen levels are reduced to about $50 \%$ of normal. Enzymatic amplification of all eight exons of the FX gene of the propositus revealed a single missense mutation( $G$ to $A)$ in exon VI resulting in a change from $\mathrm{Gly}(\mathrm{GGG})+201$ to $\mathrm{Glu}(\mathrm{GAG})$. Both parents are heterozygous for this mutation. To elucidate the mechanism which leads to the lack of FX antigen in the proposita we compared the processing of FX"VIENNA" and wild type FX in a transient expression system. Wild type and mutant FX cDNA's were expressed in the human embryonic kidney cell line 293. The nascent protein was puls labelled with 35S-Met, immunoprecipitated using a polyclonal FX antibody and analyzed on SDS-PAGE. Results showed that FC"Vienna" is produced at roughly the same amount as normal FX. It is secreted into the cell supernatant in its two chain form and is not degraded within the first 12 hours after synthesis. Addition of plasma proteases results in an signifcant reduction of FX antigen in the supernatant of the mutant construct when compared with the wild type construct. We therefore can conclude that the change from Gly+201 to Glu results in an instable protein which is rapidly degraded.

Klinik für Innere Medizin I, Abteilung für Hämatologie und Hämostaseologie, Währinger Gürtel 18-20, A-1090 Wien 
A FRAMESHIFT MUTATION IN EXON V OF THE A $\alpha$-CHAIN GENE LEADING TO TRUNCATED A $\alpha$-CHAINS IN THE HOMOZYGOUS DYSFIBRINOGEN MILANO III

M. Furlan, C. Steinmann, M. Jungo, C. Bögli, F. Fedeli, R. Redaeili, F. Baudo, B. Lämmle

An inherited abnormal fibrinogen variant, denoted as fibrinogen Milano III, was found in a 13-year-old girl suffering from recurrent venous thrombosis. Plasma of the patient exhibited prolonged thrombin time and reptilase time. Fibrinopeptide release by thrombin was normal, whereas polymerization of fibrin monomers in the presence and absence of calcium ions was strongly impaired.

SDS-PAGE of mercaptolyzed fibrinogen showed normal BB- and $\gamma$-chains, whereas no normal $\mathbf{A} \alpha$-chain was detected in the proposita. Immunoblot analysis with the monoclonal antibody $Y 18$, detecting an epitope within the stretch of amino acids $\mathrm{A} \alpha 1-51$, indicated a truncated $\mathrm{A} \alpha$-chain of about 50 $\mathrm{kD}$. Immunoblotting with antibodies directed against serum albumin demonstrated the presence of albumin covalently linked to fibrinogen.

The structural defect of fibrinogen Milano III was determined by sequence analysis of a single-stranded fragment of genomic DNA amplified by PCR. $A n$ insertion of a thymine in the exon $V$ of the $A \alpha$-chain gene after the triplet ATT coding for A $\alpha 451$ Ile altered the reading frame and generated a premature stop signal. In both parents, normal and mutant alleles were established, leading to doubling of the sequence pattern after the thymine insertion site. We conclude that the missing carboxy terminal domain of the $A \alpha$-chain in fibrinogen Milano III is responsible for the impaired polymerization of fibrin monomers.

Albumin-fibrinogen complexes in the purified fibrinogen of the proposita are most likely caused by disulfide bond formation between the free sulfhydryl group in albumin and the unpaired cysteine at position $A \alpha 442$, which is linked to $A \alpha 472$ Cys in normal fibrinogen.

Central Hematology Laboratory, Inselspital, University of Bern, CH-3010 Bern and Niguarda Hospital, I-20162 Milano

\section{3}

PROTEIN C GENE MUTATIONS IN TEN UNRELATED FAMILIES WITH SYMPTOMATIC PROTEIN C DEFICIENCY.

I. Witt, H.H. Seydewitz, D. Asbeck, S. Beck and c. Nabel

In ten unrelated families with symptomatic protein $\mathrm{C}$ deficiency we have identified the underlying gene defect. Except of one patient, who suffered from homozygous deficiency and developed severe purpura fulminans in the newborn period, all patients were heterozygotes.

We studied the DNA of the index patients and the family members by $P C R$ amplifying all nine exons including the splice site junctions and direct sequencing of the PCR products using an automated sequencer. In eight families with type I and in two families with type II protein $c$ deficiency nine different mutations were identified: eight missense and one nonsense mutation. Three of them represent novel mutations.

Most mutations were found in the catalytic ámain. One of them (H2110) changes the histidin residue of the active center and leads to type II deficiency. one of the three novel mutations (R314C) is located adjacent to the carbohydrate binding site $\mathrm{N} 313$ and also leads to type II deficiency. Another one (V325A) was found in the homozygous patient.

The novel mutation $C 141 Y$ abolishes the disulfide bond between the heavy and the light chain of the molecule. The mutation Rl69w, which was found in two families, destroys the thrombin cleavage site.

The data show that a variety of mutations is responsible for both types of protein $C$ deficiency.

*Present address: Children's Hospital, Biochemical Laboratory, University of Freiburg $i . B r .$, Mathildenstr. 1, D-79106 Freiburg i.Br. FRG
NEW MUTATIONS IN THE PROTEIN S GENE

S. Mustafa, I. Pabinger, M.Heistinger, Ch. Mannhalter

Protein S (PS) deficiency, a hereditary coagulation disorder, is detected by immunological and/or functional assays. PS levels are normally distributed in healthy as well as deficient individuals, with an overlap among both groups, which can complicate diagnosis. This drawback of functional assays is not seen with DNA analysis. Ten families with proven type I PS deficiency were tested for genomic mutations in the coding regions and exon-intron boundaries of the PS gene. In these families five mutations could be identified. a) $A, G \rightarrow A$ transition at position 5 of intron $J$ donor consensus sequence was found in all 4 affected individuals of family $A(4 / 6$, two generations), and the two affected individuals of family $B$ (two generations, not related to family A). Investigation of RNA revealed the use of a cryptic splice site, situated 32 bases upstream of the normal one. The cryptically spliced mRNA exhibits several stop codons in the new reading frame. b) A deletion of two adenins which leads to the change of lys 633 to glu, and the subsequent use of a different reading frame with the first stop codon (TAA) 21 aminoacids beyond the original stopcodon (TAA), could be identified at the end of the coding area in exon XV. The mutation was found in two unrelated families, and only in affected family members $(1$ of $2 ; 4$ of 8$)$. c) A C $\rightarrow T$ transition, which turns arg 410 into a stopcodon (TGA) was observed in the affected individuals of another family (3/5). d) A $T \rightarrow A$ transversion converting Leucin into Glutamine at position 543 and e) a $\mathrm{G} \rightarrow \mathrm{C}$ transversion turning Alanin 575 into Prolin have been identified in affected individuals, whose relatives have not yet been studied.

Klinisches Institut für Medizinische und Chemische Labordiagnostik (KIMCL), AKH, Währingergürtel 18-20, Leitstelle 5H, A-1090 Wien , Austria
65

PROTEIN C INHIBITOR (PCI): An ALLELIC POLYMORPHISM WITH DIFFERENT FREQUENCIES IN DIFFERENT ETHNIC GROUPS.

Klaus-P. Radtke, Judith S. Greengard, Bruno Villoutreix and John $H$. Griffin.

Protein $\mathrm{C}$ inhibitor (PCI), a member of the serpin super family, is heterogeneous in plasma. Heterogeneity of proteins is often attributed to differences in glycosylation. To determine if amino acid differences contribute to its heterogeneity, cDNA clones for PCI CDNAs were obtained from liver mRNA and sequenced. Two related sequences, designated allele 3.0 and allele 3.1 were obtained. The 3.0 allele was identical to the published sequence of PCI except for a silent C1153A change. Alleele 3.1 differed from allele 3.0 at 4 of 1221 nucleotides (T196C; C210T, C238T, A359G) and 2 of 387 predicted amino acids (A36V and K86E). C210T eliminated a StyI restriction site in allele 3.0. Restriction digestion was used to survey genomic DNAs of 138 individuals. The overall frequencies for allele 3.0 and allele 3.1 , were 0.64 and 0.36 respectively. By ethnic background, the frequency for allele 3.1 was 0.52 in African Americans, 0.42 in Asians and 0.14 in Caucasians. Ethnohistorical evidence and the frequency distribution among ethnic groups suggest that the $\mathbf{3 . 0}$ allele may have arisen from the 3.1 allele. In a 3 dimensional computer graphics structure of $\mathrm{PCI}$, the allele 3.1 residues. V36 and E86, were located on the molecule's surface. The K86E replacement involves a solvent exposed positive charge in helix D. Helix D appears to be directly involved in heparin binding by the homologous serpins, antithrombin III and heparin cofactor II, whereas it has been suggested that this is not the case for PCI. 3.0 allele and 3.1 altele homozygous plasmas both inhibited activated protein C (APC) with the same heparin sensitivity, consistent with the idea that helix $\mathrm{D}$ does not participate in heparin binding or stimulation for PCI. Total PCI antigen was present at similar concentrations in 3.0 allele and 3.1 allele homozygous plasmas. Quantitation of PCI:APC complexes that formed when exogenous APC was added to homozygous plasmas in the presence of heparin indicated that both types of PCI were equally functionally active APC inhibitors. Although the heparin dependent inhibition of APC by each form of PCI was indistinguishable, it is possible that these two forms of PCI may have important functional differences with other proteases. Moreover, analysis of the $\mathrm{PCI}$ genotype in various pathological conditions may help to define the functional importance of PCI forms. Present address: University Clinic Frankfurt, Center of Internal Medicine, Frankfurt, FRG 
PREVALENCE AND CLINICAL FEATURES OF PROTEIN CCOFACTOR DEFICIENCY IN 150 PATIENTS WITH A HISTORY OF VENOUS THROMBOEMBOLISM

M. Melichart, I. Hauser, P. A. Kyrle, K. Lechner, I. Pabinger

A newly described activated protein C-cofactor (APC-Cof) deficiency (def.) has recently been shown to be a risk factor for venous thromboembolism. We have investigated 150 individuals $(95 \mathrm{f}, 55 \mathrm{~m})$ currently not on oral anticoagulants with a history of venous thromboembolism. The first thromboembolic event had occurred at a median age of $41.8 \pm 14.7$ and $98 / 150$ patients (pat.) had a positive family history.

APC-Cof was determined by a test kit provided by Chromogenix (Mölndal, Sweden). APC-Cof def. was diagnosed when the addition of activated PC (APC) caused a prolongation of the PTT of less than $45 \mathrm{sec}$. APC-Cof def. was diagnosed in $48 / 150$ pat. (32\%). Pat. with a def had a mean PTT prolongation of $31.6 \pm 8.9$, the mean ratio (PTT with APC/PTT without APC) was $1.96 \pm 0.3$.

Pat. with APC-Col del. were compared to those with a normal APC-Cof value. There was no significant difference regarding the frequency of a positive family history ( $67 \%$ in pat. with APC-Cof / $65 \%$ in those without a deficiency), localisation of thrombosis and occurrence of spontanous $(58 \% / 65 \%)$ and recurrent $(46 \% / 65 \%)$ thrombosis in these two groups. The mean age of the time of the first thromboembolic event was $44 \pm 16.4$ in pat. with APC-Cof def. and $40.6 \pm 14.7$ years in those without a def. Conclusions: APC-Cof def. is a frequent finding in pat. with venous thromboembolism. However, the clinical features were not different in pat. with and without a def. state. Thus, further studies to evaluate the role of APC-Cof def. as a risk faktor for venous thromboembolism are mandatory.

Dep.of Hematology and Blood Coagulation, Med.Clin.I, University Hospital of Vienna, Austria

\section{7}

Multicenter Evaluation on various Coagulation instruments of a Kit for Activated Protein C Resistance

S.Rosen,K.Johansson.,K.Lindberg,Chromogenix,Mölndal Sweden and B.Dahlbäck,Dept. of Clinical Chemistry and Dept of Coagulation, University of Lund, Malmö, Sweden for the APC Resistance Study Group.

Recently a new hemostatic disorder has been described wich appears to be an important risk factor for familial thromboembolism. The disorder is characterized by a poor anticoagulant response to activated Protein C (APC) and has been shown to be due to lack of an APC cofactor activity which is different from Protein S.

A kit for determining the response of plasma samples to addition of APC in an APTT-based assay"COATEST APC Resistance", has been evaluated on 36 coagulation instruments in a multicenter study involving 30 laboratories. A lyophilized normal plasma and identical plasma aliquots from 20 individuals, one of whom had a borderline resistance to $A P C$, were analysed in each laboratory and the sensitivity of each plasma to APC was determined as the ratio between the clotting times obtained in the presence and absence of APC(APC ratio). The plasma from the individual with a borderline resistance to APC activity was correctly classified as the lowest responder in each laboratory, with an APC ratio in the range 1.5-2.4. In comparison, plasma from individuals with a pronounced response to APC activity resulted in APC ratios above 3.4 in most cases. The intra-laboratory coefficient of variation for the clotting times were on average $2.0 \%$ and $3.9 \%$ in the absence and presence of APC. respectively, indicating that the precision for the prolonged clotting times obtained also in the presence of APC is sufficient to allow a safe assignment of the APC response. The APC ratio for the lyophilized normal plasma was $2.7^{+} 0.2(2$ S.D.) jlllustrating a narrow distribution between instruments. Altogether, the results indicate that all the coagulation instruments included in the study can be used for detection of individuals with resistance to APC activity through determination of the APC ratio or prolongation time.
A NOVEL EXOSITE IN THE LIGHT CHAIN OF HUMAN ACTIVATED PROTEIN C ESSENTIAL FOR INTERACTION WITH BLOOD COAGULATION FACTOR Va

R.M. Mesters and J.H. Griffin

To identify regions on the surface of the light chain of activated protein C (APC) that mediate anticoagulant activity, ten synthetic peptides were prepared and tested for their ability to inhibit APC anticoagulant activity. The synthetic peptide-(142155) inhibited APC anticoagulant activity in Xa-1stage coagulation assays in normal and protein sdepleted plasma with $50 \%$ inhibition at $5-25 \mu \mathrm{M}$ peptide. In a system using purified clotting factors, peptide-(142-155) inhibited APC catalyzed inactivation of Factor $\mathrm{Va}$ in the presence or absence of phospholipids with 50 o inhibition at $50 \mu \mathrm{M}$ peptide. However, peptide-(142-155) had no effect on APC amidolytic activity or on the reaction of APC with the serpin, recombinant [ $\mathrm{Arg}^{358}$ ]-alpha ${ }_{1}$ antitrypsin. Moreover, peptide-(142-155) inhibited Factor Xa clotting activity in normal plasma as well as in a prothrombinase assay in the presence of Factor Va with $50 z$ inhibition at $5 \mu \mathrm{M}$ and $50 \mu \mathrm{M}$ peptide, respectively. Peptide-(142-155) was shown to bind directly to immobilized Factor Va. The peptide had no significant effect on Factor $\mathrm{Xa}$ or thrombin amidolytic activity and no effect on the clotting of purified fibrinogen by thrombin suggesting it does not directly inhibit these enzymes' active sites. These data are consistent with the hypothesis that the sequence of residues 142-155 in the light chain of APC provides a Factor Va binding site on APC and that peptide-(142-155) binds to Factor Va at a specific site on Factor Va, thereby interfering with both APC and Factor Xá binding to Factor Va.

The Scripps Research Institute, La Jolla, California 92037, USA.

\section{9}

POOR ANTICOAGULANT RESPONSE (APC-RESISTANCE) AMONG PATIENTS SUTFERING FROM STROKE OR DEEP VENOUS THROMBOSIS AND AMONG HEALTHY SUBJECTS W. -M. Halbmayer, A. Haushofer, R. Schön and M. Fischer.

A poor anticoagulant response to activated protein $C$ in an APTT assay (APC-resistance) was recently reported by Dahlbäck et al. to be a cause for familial thrombophilia. The response to activated protein C (APC) was measured in 30 patients suffering from juvenile or recurrent stroke, in 40 patients suffering from venous thromboembolism and in 50 healthy subjects as normal controls. The prevalence of APC-resistance was found to be significantly higher among stroke-patients $(20 \%, p<0.003)$ and patients with venous thrombosis $(17.5 \%, p<0.02)$ compared to the incidence of APC-resistance among normals $(2$ \%). In a family study 5 out of 9 investigated family members $\left(56 \frac{\circ}{5}\right)$ of a patient with deep venous thrombosis could be detected to be APC-resistant. Measuring protein $S$ activity with an automated calcium-thromboplastin-based protein $S$ activity assay a significant correlation $(p<0.0001)$ between the results of this functional protein $S$ assay and APC resistance could be calculated. Nine out of 14 patients $(64$ \%) with poor APC-response showed protein $s$ clotting activities below the normal range. We concluded that protein s clotting assays should be considered as influenced by the new APC-resistance phenomenon and considered as possibly not protein $s$ specific.

Central Laboratory and of the General Hospital Vienna-Lainz, Wolkersbergenstraße $1, \quad \mathrm{~A}-1130$ Vienna, Austria. 
INHIBITION OF ACROSIN BY SERINE PROTEASE INHIBITORS (SERPINS) AND SERPIN CONCENTRATIONS IN SEMINAL PLASMA

X.L. Zheng ${ }^{1}$, M. Geiger ${ }^{1}$, S. Ecke1, W.J. Zhang1, I. Resch ${ }^{1}$, U.

Eberspăcher ${ }^{2}$, P. Donner ${ }^{2}$, W.-D. Schleuning ${ }^{2}$, and B.R. Binder ${ }^{1}$

Acrosin, a trypsin-like serine protease present in its zymogen form in the acrosome of spermatozoa is thought to digest a pathway for the sperm through the zona pellucida of the ovum during the process of fertilization. We have shown that boar acrosin was inhibited by human protein $\mathrm{C}$ inlibitor (PCI) with an apparent second order rate constant $\left(k_{a p p}\right)$ of $5.8 \times 10^{4} \mathrm{M}^{-1} \mathrm{~s}^{-1}$. PCI is present in high concentrations in seminal plasma and endogenous $\mathrm{PCl}$ was furthermore found in the immediate vicinity of disnupted acrosomal membranes of washed human spermatozoa. This serpin could therefore function as a scavenger for prematurely activated acrosin in the male reproductive tract.

Since little is known about the interaction of acrosin with other serpin type inhibitors, we analyzed in this study the interaction of boar acrosin with other purified human serpins. Antithrombin III (ATIII), plasminogen activator inhibitor-1 (PAI-1), plasminogen activator inhibitor-2 (PAI-2), and $\alpha_{1}$ antitrypsin $\left(\alpha_{1}-\mathrm{AT}\right)$ inhibited acrosin activity. The following apparent $k_{a p} s$ were calculated: ATIII: $19.5 \times 10^{4} \mathrm{M}^{-1} \mathrm{~s}^{-1}$, PAI-1: $21.5 \times 10^{4} \mathrm{M}^{-1} \mathrm{~s}^{-1}$, PAI- 2 . $3.3 \times 10^{4} \mathrm{M}^{-1} \mathrm{~s}^{-1}, \alpha_{1}-\mathrm{AT}$ : $0.9 \times 10^{4} \mathrm{M}^{-1} \mathrm{~s}^{-1}, \alpha_{2}$-Plasmin inhibitor and heparin cofactor II did not inhibit acrosin. SDS-stable acrosin/serpin complexes were only seen with ATIII, all other acrosin inhibitors were cleaved by the enzyme. As determined by ELISAs, the concentrations of PCI, PAI-1, and PAI-2 in individual seminal plasma samples from healthy donors were $304.6 \pm 27.0 \mathrm{mg} /$, $4.4 \pm 1.2 \mu \mathrm{g} /$ and $7.5 \pm 0.8 \mu \mathrm{g} / \mathrm{l}$ (means $\pm \mathrm{SD}$ ), respectively. As judged from semiquantitative immunoblotting, ATIIl concentrations in seminal plasma were $\leq 1 \%$ of that in blood plasma (i.e. $\leq 1.5 \mathrm{mg} / 1$ ). Therefore considering both, the $\mathrm{k}_{\mathrm{app}}$ calculated for the inhibition of boar acrosin in a purified system and the concentration of each serpin in seminal plasma, PCl seems to be the best candidate to function as a physiological acrosin inhibitor in the male reproductive tract.

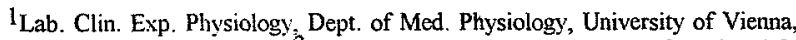
A-1090 Vienna, Austria and Institute of Cell- and Mol. Biology, Schering AG, Berlin, Germany

Vascular Disease

71

HIGHER INCIDENCE OF ELEVATED CARDIOLIPIN ANTIBODY PLASMA LEVELS IN PATIENTS DEVELOPING CORONARY RESTENOSIS AFTER ANGIOPLASTY S. Hornykewycz, M. Jörg, G. Zorn, S. Graf, B.R. Binder and K. Huber

It has been dernonstrated recently that elovated plasma leve!s of cardiolipin antibodies (antiCL) are related to thromboembolic diseases including coronary artery disoase (CAD) (Harris, Brit J Haematol 1990, 74, 1). To demonstrate a possible role for antiCL-lgG and antilgM antibodies for tho dovelopment of restenosis, we investigated 95 consecutive patients undergoing successful first coronary angiop lasty (PTCA) during 12 months follow up por iod for thair antiCL-log and antiCL-igM levels. Furtermore, wo detormined entiCL- 106 and antiCL-lgM lovels in 112 patients with CAD who oxhibited an anamnestic low (no or 1 restenosis after previous angioplasty) or high ( 22 ovents of proven restencsis) tendency for restenosis but wor in a clinically stable phase at time of investigation.

From the 95 patients after their first successful PTCA (ramaining stenosis after PTCA of $<10 \%$ vossol diamoter), 23 pationts (24\%) devoloped angiographically proven restonosis (R) within 6 months. In the romeining patients (mo restenosis. NR) control angiography was performed aftor 6 to 12 months with an oxcellent long term result. Buforo PTCA, R-patients exhibited in $30.4 \%$ olevaled antiCL$\lg 6$ lovels as compared to 8.1 X in NR-patients which was statistically significant (p<0.01). However, olevation of antiCL-loM levals did not show a significant differ ence between the study groups (R: 13\%; NR: 11.3\%). Meen plasma levels of antiCL-IgG and antiCL-lgM beforo PTCA, woll as after 3,6 , and 12 months wero not statistically significant between the groups and oxhibited for both groups very stable values over the whole follow up peried.

In pationts with high tendency for recurrent restenosis meen antiCL-lgG and anticL-IgM lovels as well as the percontages of oleveled lovels were similar as compared to patients with a low tendency for recurrent restenosis.

From theso data it is concluded that CAD pationts with olovated antiCL - Ig 6 lovols oxhibit a higher incidence of restenesis atter first successful PTCA. This finding might support the hypothesis that restenosis formation is associated with a higher thrombotic tendency. Within patients with a history of restenosis those with a high tendency for recurrent restenosis cannot be differentiated from pationts with a low tendency by antiCL-IgG or antiCL-lgM doterminations.

$2^{\text {nd }}$ Department of Internal Medicino, Division of Cardiology, University of Vienna, Währinger Gürtel 18-20, A-1090 Vienna, Austria
72

ENDOTHELIAL DYSFUNCTION AND CARDIOLIPN ANTIBODIES IN PATIENTS WITH SYSTEMIC NECROTIZING VASCULITIS O.Hergesell, K.Andrassy

Diagnosis and assessment of disease activity (DA) of small vessel vasculitis (SNV) (Wegener's granulomatosis (WG) and microscopic polyangiitis (MP) are established by measurement of ESR, CRP and anti neutrophil cytoplasmic antibodies (ANCA). In the following study we examined whether measurement of markers of endothelial cell function (von Willebrand factor (vWF) and thrombomodulin (TM)) and anti-cardiolipin antibodies (ACA) can further improve diagnosis of SNV and evaluation of DA. In a prospective study, 26 patients with WG (median age $54 \mathrm{y}$; C-ANCA median titer 1:160) and 15 patients with MP (median age $59 \mathrm{y}$; P-ANCA median titer 1:320), were analyzed upon clinical admission, and again after clinical remission. The majority of patients had impaired renal function (serum creatinine median $2,6 \mathrm{mg} / \mathrm{dl}$ ) upon clinical admission Patients with manifest infections and with manifest deep venous thrombosis (evaluated by Doppler and compression sonography) were excluded.vWF-AG (Laurell) levels were high upon clinical admission (median vWF 205\%) and remained high after CR (median vWF $189 \%$ ). Plasma TM levels (measured by ELISA (ASSERACHROM)) were high (median TM level $142 \mathrm{ng} / \mathrm{ml}$ ) in both WG and MP, although it turned out that TM levels were dependent on renal function (correlation: s-creat./ $T M=0,61$ ), $T M$ levels remained elevated, but nearly normalized with CR (median TM $70 \mathrm{ng} / \mathrm{ml}$ ). ACA (IgG/lgM maesured by ELISA) were only significantly elevated in MP (8 out of 15) but not in WG (2 out of 26) and disappeared with clinical remission. CONCLUSION: Endothelial damage in patients with SNV is demonstrated by high vWF and plasma TM. TM levels are only useful markers if renal function is considered. Presence of ACA points to a different cause of vasculopathy in MP in comparison to WG.

Present address: Department of Medicine, University of Heidelberg, Bergheimerstr. 56A, 69115 Heidelberg 
SNEDDON'S SYNDROME A SPECIAL FORM OF ANTIPHOSPHOLIPID SYNDROME (APS)?

D. Söhngen ${ }^{1}$, M. Sitzer ${ }^{2}$, I. Janda ${ }^{1}$, M. Heins ${ }^{3}$, H-C. Schuppe ${ }^{4}$, Ch. Specker ${ }^{1}$, H. Steinmetz ${ }^{2}$, E. Richter ${ }^{1}$, and W. Schneider ${ }^{1}$

$1_{\text {Dept. of Internal Medicine, }}^{2}$ Dept. of Neurology 3 Dept. of Clinical Chemistry ${ }^{4}$ Dept. of Dermatology, Heinrich-Heine-University, 40225 Düsseldorf, FAG The rare association of cerebrovascular ischemic lesions and generalized livedo racemosa was first described by the British dermatologist SNEDDON in 1965. Although clinical signs are well defined, the pathogenesis of this disorder is still obscure. Up to now about 150 patients were reported. Women aged between 20 and 40 are mostly affected. It has recently been suggested that certain antiphospholipid antibodies (APA) are associated with livedo racemosa.

Patients/Methods: To evaluate the presence of APA in patients with this disorder we examined 17 patients with Sneddon's syndrome. Females clearly dominated [16:1]. Risk factors for vascular diseases: arterial hypertension [ $n=9$ ], nicotine consumption [ $n=12$ ]; serum lipid levels tested in 13 patients showed elevated serum cholesterol in 5 , an increased ratio of LDL to HDL cholesterol in 5 patients. Two patients had a history of venous thrombosis and two fulfilled criteria for systemic lupus erythematosus. Coagulation tests to detect fupus anticoagulant (LA): activated partial thromboplastin time, Kaolin clotting time, 1:1 mixture with normal plasma, dilute Russel's viper venom test, thromboplastin inhibition test and platelet neutralization procedure; for fibrinolysis euglobulin lysis time/ELT, t-pA and PAl-1 was tested. in 13 patients additional transcranial Doppler (TCD) for microembolic monitoring of the middle cerebral artery and cardiologic evaluation was performed (electrocardiogram/ECG,Holter monitoring, echocardiography, chest X-ray, peripheral doppler sonography/PDS and venous occlusion plethysmography/VOP). Results:1.Clotting tests revealed LA in 6 patients. Prolonged ELT indicate impaired fibrinolysis in these patients although further analysis of $\mathrm{t}-\mathrm{pA}$ and PAl-1 does not show significant differences compared to control. 2.Microembolic moniloring by TCD was positive in 5 patients of whom 3 were positive for $L A$. 3.Cardiologic evaluation showed a pathologic ECG in 3 (negative $T$ wave), Holter monitoring was normal beside paroxysmal atria tachycardias in two; a systolic murmur was found in 8 patients with mitral valve prolapse and regurgitation or thickening of mitral valve (of whom 3 posifive for LA): ventricular function was normal on echocardigraphy and intracardial thrombi were excluded; cardiac thoracic ratio was pathologic in 3 patients; PDS exclude occlusion of peripheral arteries in all patients but VOP showed decreased reactive hyperemia in 9 patients which may be in accordance with disturbed microcirculation. In conclusion: Our results indicate 1.a high prevalence of LA in patients with Sneddon's syndrome indicating APS; 2.TCD may be correlated to LA;3.Cardiologic evaluation revealed a high incidence of mitral valve abnormalities. Whether LA may contribute to these findings should be clarified in further studies.

\section{4}

CHANGE OF FUNCTIONAL PROPERTIES OF VITRONECTIN BY NONENZYMATIC GLYCATION

K.T. Preissner', M. Eppinger-Albrecht', K. Benner', A. Weiss ${ }^{2}, M$. Brownles $^{3}$, and H.P. Hammes ${ }^{2}$

Diabetic hyperglycemia modifies extracellular matrix (ECM) components by forming advanced glycation end products (AGE). AGE cause qualitative and quantitative alterations that appear to contribute to vascular disease in the diabetic retina. In the present study advanced glycation of the fibrinoIytic cofactor vitronectin (VN) was characterized by structural and functional criteria. Long term incubation of human $\mathrm{VN}$ with glucose metabolites resulted in the irreversible modification of $30 \%$ lysine residues associated with higher electrophoretic mobility and the formation of SDS resistant high molecular weight products. AGE-VN was recognized by AGE-specific antibodies and was resistant to proteolysis by plasmin and trypsin. Heparin-as well as collagen-binding to AGE-VN was less than $10 \%$ of control. In contrast, plasminogen-binding and adhesive properties were hardly changed, whereas PAl-1-binding to AGE-VN even increased moderately. These in vitro findings were corroborated by histochemical analysis of retinae from 11 -month diabetic and non-diabetic wistar rats $(n=10)$. While $V N$ immunostaining was prominent and colocalized with staining for heparan sulfate proteoglycan in the internal limiting basement membrane as well as throughout the ECM in a normal population, loss of staining for both components was characteristic of diabetic retinae. Together, these data indicate that AGE-VN has lost its ability to interact with ECM components, but retaines its stabilizing function as cofactor for PAl-1. These observations are in accordance with the observed structural and functional alterations of ECM in diabetes, related to dysregulated pericellular proteolysis.

'Haemost. Res. Unit, Kerckhoff-Klinik, MPI, Sprudelhof 11, D-61231 Bad Nauheim, Germany

${ }^{2}$ Poliklinik der Justus-Liebig-Universität, Klinikstr. 36, D-35392 Giessen, Germany

${ }^{3}$ Diabetes Res. Center, Albert Einstein College of Medicine, Bronx, New York 10461, U.S.A.

\section{5}

VON WILLEBRAND FACTOR AND SOLUBLE ADHESION MOLECULES IN NON-INSULIN DEPENDENT DIABETES MELLITUS (NIDDM) K.M. Reinhardt, A.D. Blann" , B. Krammer, B. Ernst and M. Steiner

Vascular endothelial cell function and integrity are increasingly accepted to be affected in patients with diabetes mellitus. Since reliable biomarker molecules for the assessment of the vascular endothelium are yet to be defined we have investigated the integrity of the endothelium in NIDDM. Von Willebrand factor (vWf) and soluble E-selectin (SESEL) were determined as endothelial cell-specific biomarkers whereas soluble vascular cell adhesion molecule (sVCAM) and soluble intercellular adhesion molecule (sICAM) were selected as non-endothelial cell-specific products. 60 patients suffering from NIDDM were separated into two groups depending on glycaemic control. 76 healthy persons served as control group. Relative to controls, the data revealed increased vWf $(133 \pm 56$ vs $101 \pm 37$ IU/dl, $p=0.002)$, elevated sESEL $(73 \pm 33$ vs $51 \pm 17 \mathrm{ng} / \mathrm{ml}$, $p<0.001)$ and increased sVCAM $(697 \pm 292$ vs $573 \pm 173 \mathrm{ng} / \mathrm{ml}$, $p=0.003$ ). In contrast, SICAM was found to be unchanged in type II diabetics $(297 \pm 112$ vs $280 \pm 86 \mathrm{ng} / \mathrm{mt})$. Significant correlations were found between WWf and SVCAM, SESEL and SICAM and SICAM and SVCAM. No significant differences could be found between the levels in patients in good glycaemic control (glycated haemoglobin less than $7.3 \%$ ) compared to patients in poor glycaemic control. Furthermore, no correlation was established between glycated haemoglobin and vWf and soluble adhesion molecules. The results suggest that endothelial cell integrity is disturbed in patients suffering from NIDDM. Glycaemic control does not appear to influence the concentration of vWf and circulating adhesion molecules.

University of Rostock, Institute of Clinical Chemistry \& Pathobiochemistry, Heydemann-Str. 6, 18055 Rostock, Germany; University of Manchester, Department of Surgery" Manchester, United Kingdom

76

HEMOSTATIC ABNORMALITIES PERSIST DESPITE GLYCEMIC IMPROVEMENT BY INSULIN THERAPY IN TYPE 2 DIABETES MELLITUS. P.Knöbl, C.Schnack, S.Proidl, R.Prager. T.Vukovich and G.Schernthaner Type 2 diabetes mellitus is associated with disturbances of metabolic, lipid and hemostatic systems, resulting in an increased risk for cardiovascular events. In 61 patients with type 2 diabetes mellitus and secondary failure to sulfonylurea treatment (29 male, 32 female, median age 65 years, median diabetes duration 10 years) serum lipids, apolipoproteins and glycemic control parameters were determined before and 6 months after initiation of insulin therapy, as well as plasma levels of fibrinogen (CLAUSS), protein C:Ag, total protein S:Ag, D-dimer, vonWillebrand factor:Ag (ELISA) and factor VII:C (COAGULOMETRY) and compared with 45 healthy control subjects matched for age, sex and body mass index. Results (medians, Q1/Q3):

\begin{tabular}{|c|c|c|c|}
\hline METABOLIC: & control & before therapy & 6 months insulin \\
\hline $\begin{array}{l}\text { cholesterol (mg/dl) } \\
\text { triglycerides (mg/dl) } \\
\text { HDL-chol. (mg/d) } \\
\text { LDL-chol. (mg/dl) } \\
\text { apolipoprot.A1 (mg/dl) } \\
\text { apolipoprot.B (mg/di) } \\
\text { HbAtc (\%) } \\
\text { fructosamine (mmol/L) } \\
\text { glucose (mg/dl) }\end{array}$ & \begin{tabular}{|ll}
233 & $(210 / 269)$ \\
128 & $(82 / 163)$ \\
61 & $(48 / 78)$ \\
153 & $(125 / 166)$ \\
175 & $(145 / 211)$ \\
128 & $(109 / 145)$ \\
$5.1(4.5 / 5.7)$ \\
$2.7(2.3 / 3.1)$ \\
$93(85 / 102)$ \\
\end{tabular} & $\begin{array}{l}226(195 / 273) \\
159(115 / 251)^{2} \\
41(34 / 53)^{3} \\
131(106 / 160) \\
132(116 / 179)^{3} \\
146(120 / 167)^{1} \\
10.1(9.1 / 11)^{3} \\
4.0(3.6 / 4.4)^{3} \\
208(165 / 241)^{3}\end{array}$ & $\begin{array}{l}235(198 / 273) \\
120(104 / 160)^{a} \\
57(41 / 68)^{b} \\
153(115 / 172) \\
170(149 / 206)^{b} \\
133(103 / 160)^{2} \\
7.5(5.5 / 8.5)^{2 c} \\
3.0(2.6 / 3.7)^{3 c} \\
139(112 / 170)^{3 b} \\
\end{array}$ \\
\hline \multicolumn{4}{|l|}{ HEMOSTASIS } \\
\hline $\begin{array}{l}\text { fibrinogen }(\mathrm{mg} / \mathrm{d} \text { ) } \\
\text { factor Vil:C (U/L) } \\
\text { D-dimer } \mu g / \mathrm{L}) \\
\text { protein C:Ag (U/L) } \\
\text { tot.protein } \mathrm{S}: \mathrm{Ag}(\mathrm{U} / \mathrm{L}) \\
\text { vonWillebr.:Ag (U/L) }\end{array}$ & $\begin{array}{l}286(258 / 304) \\
0.9(0.8 / 1.1) \\
86(47 / 124) \\
1.0(0.8 / 1.1) \\
0.9(0.8 / 1.1) \\
1.3(1.1 / 1.5) \\
\end{array}$ & $\begin{array}{l}317(287 / 365)^{1} \\
1.1(0.9 / 1.4)^{2} \\
105(71 / 178)^{1} \\
1.2(1.1 / 1.4)^{3} \\
1.2(1.0 / 1.3)^{3} \\
1.6(1.2 / 2.0)^{2}\end{array}$ & $\begin{array}{l}321(281 / 350)^{1} \\
1.0(0.9 / 1.1)^{1} \\
114(64 / 238)^{1} \\
1.3(1.0 / 1.4)^{3} \\
1.1(0.9 / 1.4)^{3} \\
1.6(1.1 / 2.4)^{1}\end{array}$ \\
\hline
\end{tabular}

vs. control: $1 \mathrm{p}<05,2 \mathrm{p}<01,3 \mathrm{p}<.001 \quad$ vs. pretreatment: a $\mathrm{p}<.05, \mathrm{~b} \mathrm{p}<01, \mathrm{c} \mathrm{p}<001$ Before initiation of insulin therapy patients had significantly elevated levels of atherogenic lipid fractions and decreased levels of "protective" factors, plasma levels of hemostatic factors were significantly higher than control values. This constellation indicates an atherogenic and hypercoagulable state, associated with endothelial cell damage. Insulin therapy results in a significant reduction of metabolic parameters, but the abnormal hemostatic values remained elevated. Therefore, metabolic optimisation does not necessarily result in a reduction of hemostatic disturbances and cardiovascular risk in type 2 diabetic patients. 


\section{PLASMIN ACTIVATES HUMAN COAGULATION FACTOR $X$ POSSIBLE INTERLINK WITH RETHROMBOSIS AFTER THROMBOLYSIS?}

Yu.V. Jitkova and G.V. Bashkov

The development of vascular reocclusion after termination of thrombolytic therapy may be connected with the activating effect of forming plasmin $(\mathrm{Pm})$ on blood coagulation proteins. We studied the capability of $\mathrm{Pm}$ to activate human coagulation factor $X(F X)$. Human $\mathrm{Pm}$ was incubated at $37^{\circ} \mathrm{C}$ with purified $\mathrm{FX}$ and quantity of forming FXa was determined by the hydrolysis of S-2222 after Pm inhibition by aprotinin. Activation of FX by Pm occurred in the presence of anionic phosphoipids $(\mathrm{PL})$ and $\mathrm{Ca}^{2+}$ ions only. At the saturation concentration of PL Pm activated $\mathrm{FX}$ with $\mathrm{Km}=1.9 \mu \mathrm{M}$ and $\mathrm{k}_{\text {cat }}=5 \cdot 10^{-2} \mathrm{M}$ in $0.05 \mathrm{M}$ Tris- $\mathrm{HCl}, 0.15 \mathrm{M} \mathrm{NaCl}, 5 \mathrm{mM} \mathrm{CaCl}_{2}$, $\mathrm{pH}$ 7.4. $\varepsilon$-aminocaproic acid inhibited $\mathrm{Pm}$-induced activation of $\mathrm{FX}$ with $\mathrm{k}_{\mathrm{f}}<10 \mathrm{mM}$. Coagulation factor VIII did not influenced on the activation of $\mathrm{FX}$ by $\mathrm{Pm}$. In the contrast, tissue factor (TF) dramatically increased $\mathrm{Pm}$-induced activation of FX. The low admixture of coagulation factor VIla $(<0.1 \mathrm{IU} / \mathrm{ml})^{\star}$ in human TF

$\begin{array}{lcc}\text { Composition } & \mathbf{V}_{\mathbf{0}}, \mathbf{M} / \mathbf{L} \cdot \min & \mathbf{F X} \mathbf{a}_{\max }, \mathbf{n M} \\ P m+F X+P L+C a 2+ & 1.06 \cdot 10^{-9} & 18 \\ P m+F X+T F+C a 2+ & 9.8 \cdot 10^{-9} & 26 \\ P m+F X+V I^{*}+C a 2+T F & 63 \cdot 10^{-9} & 108\end{array}$

induced a six-fold increase in a velocity of $F X$ activation. In the presence of $\mathrm{PL}$ and $\mathrm{Ca}^{2+} \mathrm{Pm}$ activated $3 \%$ of $\mathrm{FX}$, but in the presence of TF and $\mathrm{Ca}^{2+} 16 \%$ of FX converted to FXa. The comparison of catalytic efficiencies $\left(V_{\text {max }}: \mathrm{Km}\right)$ clearly suggests that at the initiation of the extrinsic pathway of coagulation $\mathrm{Pm}$ is a 60 -fold more effective activator of FX, than in the presence of intrinsic $\mathrm{X}$-ase components.

Lab. Blood Coagulation, National Haematological Scientific Center, Novo-Zykovski pr. 4A, Moscow 125167, Russia.

78

SECOND MESSENGERS IN THROMBIN- AND TRAP-INDUCED CONTRACTION OF PORCINE PULMONARY ARTERY

E. Bretschneider, M. Paintz, and G. Glusa

Recently it was shown that thrombin cleaves a peptide from the extracellular $\mathrm{N}$-terminus of its receptor and that the newly generated $\mathrm{N}$ terminus can act as a tethered ligand to activate the receptor. The following $\mathrm{PIP}_{2}$-hydrolysis results in the generation of two second messengers; inositol 1,4,5-triphosphate $\left(I_{3}\right)$, which releases $\mathrm{Ca}^{2+}$ from intracellular stores and diacylglycerol, which activates proteinkinase $C$ (PKC). A synthetic peptide of 14 amino acids corresponding to the sequence of the new N-terminus (TRAP-14) was found to possess thrombin-like activity. We investigated the involvement of intracelluiar messengers in the contractile effect of thrombin and TRAP-14 in endothelium- denuded porcine pulmonary arteries. Both agonists induced a sustained contractile response and a temporary increase in $I_{3}$. The TRAP-induced increase in $\mathrm{IP}_{3}$ and force development did occur faster compared to thrombin. In order to evaluate the role of PKC for the contractile response of porcine pulmonary arteries the PKC was activated directly by phorbol 12,13-dibutyrate (PDBu, $50 \mathrm{nmol} / \mathrm{l})$ Furthermore, the effect of the PKC inhibitor staurosporine $(50 \mathrm{nmol} / 1)$ was investigated. Preincubation of the vessels with staurosporine inhibited the PDBu-induced sustained contraction by about $80 \%$. In the presence of staurosporine the thrombin and TRAP-induced rise of $\mathrm{IP}_{3}$ and the phasic contraction were still evident, whereas the tonic contraction was significantly inhibited. Thus, $\mathrm{IP}_{3}$ seems to be responsible for the phasic component and the actvated PKC for the tonic component of thrombin- and TRAP-induced contractions.

Institut of Pharmacology and Toxicology, Medical School Erfurt, Nordhäuser Str. 74, D-99089 Erfurt FRG
THE ROLE OF FIBRINOGEN IN TWO MODELS OF ARTERIAL THROMBUS FORMATION: FIBRINOGEN-RECEPTOR BLOCKADE VERSUS INHIBITION OF FIBRINOGEN CLEAVAGE AND FIBRINOGEN DEPLETION K. Rübsamen , W. Homberger, M. Kirchengast.

The initial steps of arterial thrombus formation are platelet adhesion to injured endothelium, fibrinogen-dependent aggregation accompanied by thrombin generation and conversion of soluble fibrinogen into insoluble fibrin. The possibility of a therapeutic intervention at various stages of these fibrinogen-mediated processes has been experimentally evaluated with antibodies or peptides blocking the fibrinogen receptor on platelets, and with compounds inhibiting fibrinogen binding to thrombin and its subsequent cleavage, such as hirudin. So far only few informations are available on the antithrombotic potential of compounds lowering fibrinogen plasma concentrations such as arwin, a thrombin-like serin protease isolated from the Malayan pit viper venom. The aim of this study was to directly compare these three principles of action in different arterial thrombosis models

Cyclic blood flow reductions (CFRs) were induced in the left coronary artery(LAD) of mongrel dogs by mechanical injury of the endothelium combined with critical stenosis. Complete suppression of CFRs was observed in animals treated with 0.5 $\mathrm{U} \mathrm{kg}^{-1}$ arwin when fibrinogen level fell below $500 \mathrm{mg}^{-1}$ and also after thrombin inhibition with hirudin ( $\left.1 \mathrm{mg} \mathrm{kg}^{-1}+1 \mathrm{mg} \mathrm{kg}^{-1} \mathrm{~h}^{-1}\right)$. Blockade of the fibrinogen receptor with the GPIIb/IIla-antagonist Ro 43-8857 $\left(0.1 \mathrm{mg} \mathrm{kg}^{-1}+0.1 \mathrm{mg} \mathrm{kg}^{-1} \mathrm{~h}^{-1}\right)$ did not completely abolish CFRs in this model.

In another set of experiments thrombus formation was induced in the canine carotid artery by insertion of a copper coil. Reperfusion was achieved in all control animals after $40 \pm 7 \mathrm{~min}$ by infusion of $\mathrm{rt}-\mathrm{PA}\left(80 \mu \mathrm{g}+8 \mu \mathrm{g} \mathrm{kg}{ }^{-1} \mathrm{~min}^{-1}\right)$. With concomitant arwin treatment the time until recanalization was significantly shortened to $21 \pm 7$ $\mathrm{min}$. In contrast, infusion of Ro 43-8857 even slightly increased reperfusion time. Mean carotid artery blood flow was maintained at a high level in the arwin- and hirudin-treated groups during the observation time of $4 \mathrm{~h}$ after termination of rt-PAinfusion. Ro-43-8857 showed a significantly smaller effect although ADP- and thrombin-induced platelet aggregation measured ex vivo in the same animals indicated complete blockade of platelet function.

The results obtained in these arterial models show that both, CFRs as indicators of platelet adhesion and aggregation, and occlusive thrombus formation, are potently inhibited by arwin and hirudin whereas the blockade of fibrinogen binding to platelet GPIIb/llla alone represents a less potent antithrombotic principle.

Knoll AG, Dept. of Cardiovascular Pharmacology, D-67008 Ludwigshafen

80

EVIDENCE FOR THE ROLE OF TISSUE FACTOR PATHWAY INHIBITOR (TFPI RELEASE IN THE MEDIATION OF THE ANTITHROMBOTIC ACTIONS OF LOW MOLECULAR WEIGHT HEPARINS (LWMHS) AND A SYNTHETIC SULFATED LACTOBIONIC ACID DERIVATIVE. W. Jeske, A. Kammereit, R. Klauser, W. Raake, P. Eckenberger, D. Hoppensteadt, and J. Fareed. Loyola University Medical Center, Maywood, II and Luitpold-Pharma, Munich, Germany.

LMWHs have been shown to produce some of their prophylactic antithrombotic actions by non-AT-1II mediated mechanisms. Previous reports have also suggested that $\mathrm{LMWHs}$ and a synthetic lactobionic acid amide derivative, aprosulate (Luitpold-Pharma, Munich, Germany) produce dose and time dependent increase in the levels of functional TFPI in patients and human volunteers. In order to further study the effect of various LMWHs and aprosulate on the release of TFPI, we employed a sensitive sandwich ELISA method (American Diagnostica, $\mathrm{CT}$ ) and a functional, chromogenic method to quantitate TFPI antigen and functional activity. Serial blood plasma samples were collected from groups of human volunteers and patients in several clinical trials and TFPI levels were measured. The TFPI antigen levels were expressed as fold increase relative to baseline using a full length recombinant TFPI (Monsanto, St. Louis, MO) as an external calibrator. The functional activity was expressed in terms of the relative inhibition of $F X_{a}$ in reference to the pretreatment sample. LMWH's in prophylactic dosages $(0.5$ to $1.0 \mathrm{mg} / \mathrm{kg} 0 . \mathrm{d}$.) were found to produce a time-dependent increase in TFPI levels within 1 to 2 hours which sustained for up to 8 hours. Repeated administration of LMWH resulted in corresponding increases in the TFPI level for a period of treatment up to 7 days. In contrast to the immunologic levels, relatively milder increases in the functional TFPI were noted. However, the time course approximated the TFPI antigen levels. In a study with escalating doses of aprosulate $(0.25$ to 2.0 $\mathrm{mg} / \mathrm{kg}$ ) progressive dose-dependent increases in the TFPI antigen and functional activity was evident. In a secand study, 3 groups of human volunteers were administered $35 \mathrm{mg}$ b.i.d., $70 \mathrm{mg}$ b.i.d., or $70 \mathrm{mg}$ o.d. aprosulate. Blood samples were drawn for up to 9 days. A marked increase in the TFPl antigen level was observed in all three groups. The $70 \mathrm{mg}$ b.i.d. group showed the strongest effect as up to a 6 fold increase was evident in some of the samples. A dose-dependent effect on the TFPI antigen levels was noted. This data clearly suggests that LMWHs and aprosulate increase circulating TFPI antigen and functional levels which may contribute to their observed antithrombotic actions. While this data indicate a plasmatic increase in the TFPI level after LMWH and aprosulate administration, the role of the agent in the modutation of vessel bound TFPI remains to be elucidated. 
UROKINASE-TYPE PLASMINOGEN ACTTVATOR IS PARTIALLY PROTECTED FROM INACTIVATION BY THROMBIN AND THROMBIN / THROMBOMODULIN COMPLEX WHEN BOUND TO ITS RECEPTOR (UPAR, CD 87).

S. Wilhelm, O. Wilhelm, U. Weidle*, M. Schmitt, and H. Graeff.

Thrombomodulin (TM), a transmembrane receptor on endothelial cells, binds thrombin in a $1: 1$ complex. Upon binding to TM the serine protease thrombin is less efficient in clotting of fibrinogen, activation of factor $\mathrm{V}$, VIII and platelets. The thrombin / TM complex activates protein $\mathrm{C}$ on the endothelial cell surface. The single-chain form of urokinase (pro-uPA) is a substrate for thrombin and cleaved at the Arg 156 - Phe157 bond, leading to an inactive two chain form of urokinase. This inactivation of pro-uPA is accelerated 70-fold in the presence of TM. Upon binding to a high affinity receptor (UPAR, CD 87) on the cell surface pro-uPA is activated by plasmin, cathepsin $B$ / $L$ and plasma kallikrein to the active two chain form which activates plasminogen to plasmin. The present study investigated whether urokinase when bound to uPAR is still inactivated by thrombin and / or the thrombin / TM complex.

To study the in vitro effects of uPAR on the inactivation process a soluble urokinase receptor (rec-uPAR) was cloned and produced in CHO-cells and purified to homogeneity by affinity chromatography. $50 \%$ inactivation of pro-uPA (500 ng / $\mathrm{ml}$ ) by thrombin occurred at $400 \mathrm{ng} / \mathrm{ml}$ of thrombin in the absence of rec-uPAR and at $800 \mathrm{ng} / \mathrm{ml}$ of thrombin when pro-uPA was bound to rec-uPAR. In the case of the inactivation of pro-uPA by the thrombin / TM complex four-fold higher concentration of the complex was necessary to achieve $50 \%$ inactivation of pro.uPA when bound to its receptor ( 210 versus $55 \mathrm{ng} / \mathrm{ml}$ of thrombin / TM complex ). At fixed concentrations of pro-uPA, thrombin or thrombin / TM complex concentration the addition of rec-uPAR restored in a concentration dependent manner uPA activity. Rec-uPAR is not cleaved by thrombin or thrombin / TM complex at the experimental conditions used as judged by SDS-PAGE.

In conclusion, pro-uPA when bound to its cell surface receptor is partially protected from inactivation by thrombin and the thrombin / TM complex and substantiates the important role of the urokinase receptor in cell-associated fibrinolytic processes.

Frauenklinik der TU München, Klinikum rechts der Isar, Ismaningerstr. 22, D81675 München, and *Boehringer Mannheim, Penzberg, Germany.

\section{Endothelial Cell}

82

THROMBIN RECEPTOR PEPTIDE RELEASES VON WILLEBRAND FACTOR FROM HUMAN UMBILICAL VEIN ENDOTHELIAL CELLS (HUVEC)

\section{J. Storck, H.Bertram and R.E. Zimmermann}

The main function of thrombin is the proteolytic conversion of fibrinogen to fibrin. Also it is well known that the addition of thrombin to HUVEC induces rising in the concentration of von Willebrand factor ( $v W f$ ) in the supernatant.

The present study was undertaken to define clearly the receptor, which is responsible for the thrombin induced vWf release from HUVEC. Vu et al. reported that cleavage of the platelet thrombin receptor by thrombin resulted in a new N-terminus (SFLLRN...) which acts as tethered ligand. The free peptide activates platelets and induce rises in both cytosolic free $\mathrm{Ca}^{2+}$ and $\mathrm{PGI}_{2}$ production in HUVEC (Cell 64, 1057, 1991). HUVEC were incubated $1 \mathrm{~h}$ with thrombin, trypsin, SFLLRN, and in inhibition experiments with the thrombin receptor inhibitor peptide FLLRN. After incubation the intracellular vWf content was compared with the vWf concentration in the supernatant. The intracellular $\mathrm{vWf}$ concentration was measured by microscope fluorometry and the concentrations in the supernatant with an ELISA. The thrombin stimulated cells showed $53 \%$ vWf antigen compared with non stimulated control cells (100\%). This result was well correlated with the 2.4 fold higher vWf concentrations measured in the supernatant of thrombin stimulated cells than in the control cells. Also the addition of SFLLRN $(0.1-200 \mu \mathrm{M})$ or trypsin $(0.1-2$ $\mathrm{nM}$ ) increased $\mathrm{vWf}$ release from HUVEC in a dose dependend manner, whereas FLLRN decreased the effects of the activators mentioned above. This results indicate that thrombin induced vWf release from HUVEC is mediated through the activation of the tethered ligand receptor.

Universität Münster, Institut für vegetative Physiologie, Robert Koch Straße $28,-48149$ Münster
83

NITRIC OXIDE SYNTHESIS IS IMPAIRED IN GLUTATHIONE DEPLETED FNDOTHELIAL CELLS R.Heller, D.Ghigo*, P.Alessio*, F.Bussqlino*, C. Costamagna*, G.P.Pescarmona*, A.Bosia* and U. Till

Human umbilical vein endothelial cells (HUVEC) were tested for their ability to synthesize nitric oxide (NO), which has been identified as endothelium-derived relaxing factor. The synthesis of No (detected as citrulline, which is produced stoichiometrically + with no from arginine) in HUVEC is $\mathrm{Ca}^{++}$-dependent, is increased 7 fold by the calcium ionophore ionomycin ( $2 \mu \mathrm{M})$ and accounts for most basal and ionomycin-induced cGMP formation. Loading of cells with reduced glutathione (GSH) by incubation with $5 \mathrm{mM}$ GSH or 5-10 mM GSH monoethyl ester led to increased basal and ionomycininduced citrulline production. When the cells were depleted of GSH by incubation with 1-chloro2 ,4-dinitrobenzene (CDNB, 2-200 $\mu \mathrm{M}$ ), basal and ionomycin-stimulated citrulline synthesis were inhibited in a concentration-dependent way. This was accompanied by a decrease of basal and ionomycin-induced but not of sodium nitroprusside-elicited cGMP formation. Intracellullar levels of $G S H$ and citrulline showed a close correlation $(r=0.992)$. The block of citrulline synthesis by CDNB was relieved when cells were replenished with GSH. N-(2mercaptopropionyl)-glycine, a permeant sulfhydry 1 compound, had no effect on citruliine levels and did not prevent cDNB-induced inhibition of citrulline synthesis. These results suggest a specific requirement of No synthesis in HUVEC for GSH which could play a role as a cofactor or prevent No synthase from inactivation by No or other radicals.

Institute of Pathological Biochemistry, school of Medicine, 99012 Erfurt, FRG, "Department of Genetics, University of Torino, 10126 Torino, Italy. 


\section{4}

MECHANISM OF AGE'S MEDIATED TISSUE FACTOR INDUCTION IN ENDOTHELIAL CELLS

A. Bierhaus, O. Vettermann, N. Mackman, M. Haase, M. Müller, R. Ziegler, D.M. Stern, P. Wahl, P.P. Nawroth

Incubation of endothelial cells (EC) with AGE's (advanced glycated end products) leads to a time and dose dependent increase of tissue factor (TF). Promotor analysis of TF demonstrated, that both AP-1 binding sites of the gene are involved in TF induction, however in contrast to stimuli as TNF not only the known $\mathrm{NF}-\mathrm{KB}$ site, but an additional NF-KB like site seems to mediate TF induction. Deletion of the promotor region from bp -948 to bp 274 does not reduce $\mathrm{TF}$ induction by TNF, however by AGE's. In this region we identified a second potential NF-KB site EMSA showed time and dose dependent increases in AP-I and $N F-K B$ binding to both $A P-1$ and both NF-KB sites. Induction could be blocked by transient overexpression of truncated jun of IkB.

Induction was dependent on the receptor for AGE's (RAGE), since blocking RAGE synthesis with antisense oligonucleotides to a highly conserved region of RAGE abolished RAGE expression and TF induction by AGE's.

85

DELETION OF THE SP-1 REGION ABOLISHES INDUCIBILITY OF TISSUE FACTOR BY GROWTH FACTORS

M. Haase, A. Bierhaus*, N. Mackman\#, T. Luther, S. Albrecht, C. Flössel, M. Müller, P.P. Nawroth*

In MCF-7 mammary tumor cells (EGF receptor positive subline), EGF or TGFalpha not only stimulates growth but also expression of tissue factor (TF). The maximum of protein expression is after $\mathbf{6 h}$ (ELISA, clotting), the maximum of mRNA expression is after $2 \mathrm{~h}$ (northern blot).

The TF promotor contains binding sites for NFKB, AP-1 and SP-1. NFkB and AP- 1 are of pivotal importance in mediating TF induction by inflammatory mediators. When MCF-7 cells were stimulated with growth factors (EGF or TGFalpha), AP-1 and NFkB were not as central as in models of TF induction by inflammatory mediators. Overexpression of NFkB ( $\mathbf{p} 65$ ) or AP-1 by transient transfection only moderately increased TF expression. Consistently deletion of the AP-1 or NFkB binding sites in tissue factor promotor mutants reduced TF baseline expression and induction by growth factors, but did not abolish it. Deletion of the SP-1 binding sites in the tissue factor promotor completely blocked TF induction by growth factors. Therefore TF induction by growth factors in MCF-7 cells is mainly dependent on intact SP-1 like binding sites, while baseline expression is dependent on AP-1 and NFkB, possibly in concert with a basal amount of SP-1 expression. Hence induction of TF by infammatory mediators in macrophages and endothelial cells is mediated by different transcriptional pathways, than induction by growth factors in tumor cells.

Technical University of Dresden, Dept. of Pathology, Fetscherstr. 74, 01307 Dresden, Germany, Fax 0351-4584328

* Univ. of Heidelberg, Germany

\# La Jolla, Univ. of California, USA
REGULATION OF THE TISSUE FACTOR GENE IN HUMAN ENDOTHELIAL CELLS AND CELL LINES

D. von der Ahe, B. Jost, C. Kunz, and G. Müller-Berghaus

Tissue factor (TF), the major cellular trigger of the coagulation protease cascade is not expressed on the luminal site of the endothelial cells under physiological conditions. However, TF expression can rapidly be induced in cultured human umbilical vein endothelial cells (HUVEC) by several agonists including phorbol ester (TPA) and tumor necrosis factor (TNF $\alpha$ ). Stimulation of HUVEC by TNF $\alpha$ or TPA results in rapid, dose-dependent increase in both TF mRNA and functional activity. Our experiments demonstrate that the increase in steady-state levels of TF mRNA was maximal at $2 \mathrm{~h}$ and was independent of de novo protein synthesis. To directly identify the TF promoter elements necessary for induction of TF in HUVEC, HeLa and NIH 3T3 cells, cells were transiently transfected with plasmids containing the human TF promoter cloned upstream of the chloramphenicol acetyltransferase (CAT) gene. It was found that the TF promoter contains two AP-1 sites, a distal (AP-1d:-217 to -223) and a proximal site (AP-1p: -204 to -210 ), which are bound by Fos-Jun heterodimers in vitro. NF-KB site $(-172$ to -190$)$ was found. Deletion of the distal AP-1 site (-213TF-CAT) leads to a 4 to 5 -fold increase in basal level TF promoter activity in HUVEC and to a lesser extend in HeLa and NIH $3 \mathrm{~T} 3$ cells. In HUVEC, however, there is no induction of this construct by TNF $\alpha$ or TPA, whereas in HeLa and $\mathrm{NIH} 3 \mathrm{~T} 3$ cells there is a strong induction by these agonists. Deletion of both AP-1 sites (-194TF-CAT) reduces the TF promoter activity more than 20 -fold, and the induction is completely abolished. This suggests that the NF-KB site does not function in the intact TF promoter without the upstream AP-1 sites. Combinatorial clustered point mutations within the AP-1 sites and the NF-kB site showed that the AP-1d and AP-1p sites can influence each other, and that they act differently in concert with the NF-KB site for full promoter induction. Furthermore, our data suggest that an additional gene regulatory repressor-like activity might be located either upstream or downstream of the TF promoter in HUVEC.

Hemostasis Research Unit, Kerckhoff-Klinik, MPI, Sprudelhof 11, D-61231 Bad Nauheim, Germany

\section{7}

DIFFERENT FORMS OF TFPI ARE RELEASED BY A HUMAN ENDOTHELIAL CELL LINE

M. Kalbas, T. Hofmann, U. Hinz, and G. Müller-Berghaus

The regulation of the extrinsic pathway of coagulation involves a Tissue Factor Pathway Inhibitor (TFPI). As predicted from the c-DNA sequence, TFPI is a protein of $32 \mathrm{kDa}$, and consists of three Kunitz-type inhibitory domains. Previous studies indicate that TFPI is present in human plasma in two forms with a molecular weight of $34 \mathrm{kDa}$ and $40 \mathrm{kDa}$.

Using monoclonal and polyclonal antibodies to human TFPl, we characterized the secretion of TFPI by a human endothelial cell line, Eahy 926. TFPI activity reached a plateau after $24 \mathrm{~h}$ at $250 \mathrm{mU} / \mathrm{ml} / 5 \times 10^{5}$ cells. Stimulation with TNF, 1/ 1-B or hirudin had no effect on the TFPI secretion, but TFPI activity increased three-fold during heparin incubation (10 IU heparin $/ 5 \times 10^{5}$ cells). Analysis of conditioned media of endothelial cells by immunoblotting detected two distinct bands of $40 \mathrm{kDa}$ and $43 \mathrm{kDa}$. Incubation of EAhy 926 in the presence of tunicamycin reduced the activity to $50 \%$, and two non-glycosylated bands of $34 \mathrm{kDa}$ and $37 \mathrm{kDa}$ were detectable. The addition of cycloheximide reduced TFPI activity to only $30 \%$ in contrast to a complete reduction of the $40 \mathrm{kDa}$ and $43 \mathrm{kDa}$ forms, as detected by western blotting with a monoclonal antibody. In contrast to these findings, we detected major bands of $35,40,43,49$ and $54 \mathrm{kDa}$ in the conditioned media of EAhy 926.

The present results indicate that different forms of TFPI are secreted by the human endothelial cell line EAhy 926.

Haemostasis Research Unit, Max-Planck-Institut für physiologische und klinische Forschung, Kerckhoff-Klinik, D- 61231 Bad Nauheim 
Tissue factor pathway inhibitor (TFPI) bound to FXa inhibits the catalytic activity of the tissue factor (TF)/factor VII (FVII) complex. Previous studies indicate that the first Kunitz type domain of TFPI is involved in the binding of TFPI to FVlla. Using monoclonal antibodies to human FVII and TFPI, we now identified a corresponding region on the FVII molecule.

Purified human FVII/FVlla alone, or in complex with TF, was coated on microtiter plates and incubated with TFPI, or TFPI and FXa. Bound TFPI was subsequently detected with monoclonal antibodies to TFPI, a PODlabelled goat anti-mouse antibody, and ABTS. The highest binding capacity of TFPI to FVII/FVIla was observed in the presence of FXa and TF. This binding could be inhibited dose-dependently with the $F(a b)$-fragment NM15 reacting with an epitope localized on the heavy chain of FVII (residues 329 356). This epitope includes the active site serine residue at position 344 . Four different antibodies to FVII and an antibody to human serum albumin did not interfere with TFPI for binding to FVII.

The same results were obtained in a ligand blot. Native FVII, active $\alpha$ FVIla and inactive BFVlla were immobilized on nitrocellulose after SDS-PAGE under reducing conditions. TFPI bound to the single-chain molecule of native FVII as well as to the heavy chain of FVIla and the small fragment of inactive BFVla all containing the active site serine residue. The binding could be blocked with the $F(a b)$-fragment NM15 which binds to FVII residues $329-356$.

The presented results indicate that an epitope on the FVII molecule containing the amino acids $329-356$ is involved in the binding of FVII to TFPI.

Hemostasis Research Unit, Max-Planck-Institut für physiologische und klinische Forschung, Kerckhoff-Klinik, Sprudelhof 11, D-61231 Bad Nauheim, Germany.

\section{Prethrombotic State}

89

QUANTITATIYL EVALDATION OF IN VTVO THKOMFIN GKNERATION BY ASSAYS FOR FIRRIN

C.E.Demprle*, S.Pnizner, M.Dollinan, H.Lill, W.Muldhuler, A.Dcssauer, K.Fassbender, D.L.Heene

Various ussay systems have been proposed for estimation of iftravasal thrombin activity. Since active thrombin is sapidly bound by inhibitors such as $\Lambda T$ IIT, or adsorbed to tibrin, specific products generated during prothrombin accivation or catalytic action of thrombin have to be moasured. Activation peptides, such as prothrombin fragment $F 1+2$, of fibrinopeptides display rapid renal elimination, which limits their usefulness for clinical diagnosis of acute coagulopathies. Earmyme-intibitor complexes, e.g. TAT are dependent upon presence of inhibitors and cofactors (heparin), resulting in quite variable results. Soluble derivatives of major substrates of throm bin with long plasma half life, such as fibrin monomer directly reflect thrombin activity. Fibrin monomers appear in circulation as complexes of variable size with fihrinogen and car be measured after desaggre vation with thiccyanate by an ELISA prosedure. Thiocyanate treatment results in a stable monomeric analyte with fihrin-specific neo-epitopes accessible to specific monoclonal antibodies. The assay has been employed for diagnosis and monitoring of clotting activation in patients with venous thrombosis, septicaemia, ccrehral ischemic insult, DIC. liver cinthosis and portocaval shunt, and librinolytic therapy for myocardial infarction. Preliminary resulrs show good correlation of fibrin monomer ELISA with fibrin fragmen D-dimer, which is generaled by plasmin degradation of crosslinked fibrin clots $(R=0.80-0.95$ in various patient groups). Correlation of the ELLSA with functional tests for soluble finrin complexes is poor $(R=0.40-0.65)$ due to the variability thesc

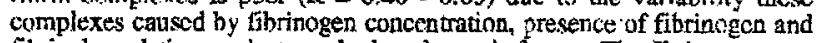
fibrin degradation products, and other plastratic factors. The fibrin monomer ELISA is not influenced by presence of fibrinogen degradation products or by blood sampling via intravenous or intranterial cuthotors. Nommal rangc values are below $3.6 \mu \mathrm{g} / \mathrm{ml}$. Values observed in patien 1 with acute. venous thrombosis or pulmonary embolism are belwoon 50 and $500 \mu \mathrm{g} / \mathrm{ml}$. Similar values are found in plasma of patients with acute DIC. Fffectivc anticoagalation lowcrs fibrin monomer levels to nornal range. Apart frow human plasma. the specificity of the kuonoclonal antibodies allows measurement of fibrin monomers from rat, horse pio, and bovinc plasma, although this rcquires species-specific reference curves, allowing its use in various animal models of thrombosis of intavaseular codsulation. *Universität Heidelbcre, Klinikum Mannheim, I.Med.Klinik, Theodor Kutzer Ufer, D-68:67 Mandein
HIGH PREDICTIVE VALUE OF D-DIMER LEVELS FOR OCCURRENCE OF DEEP VEIN THROMBOSIS AFTER HIP ARTHROPLASTYY

I. Bodamer, w. Stenzinger, and J. van de Loo

Since there is little information about the predictive value (PV) of D-Dimer concentrations in patients (pts) with or without postoperative deep vein thrombosis (DVT) under prophylactic antithrombotic treatment, plasma samples from pts undergoing hip arthroplasty were collected on day (d) 3 and $d 10$ after surgery and tested for $D-$ Dimer levels by enzyme immunoassay. All pts were treated with unfractionated heparin (UH) or low molecular weight heparin (LMWH:CY216) in a doubleblind randomized multicenter trial (GHAT, Arch Orthop Trauma Surg, 1992). Presence of DVT was assessed by bilateral phlebography on d $14 \pm 1$. Postoperative D-Dimer levels of pts with DVT (d 3 : $\mathrm{n}=63, \bar{x}=1925 \mathrm{ng} / \mathrm{ml}$; $d$ 10: $n=50, \bar{x}=2488 \mathrm{ng} / \mathrm{ml}$ ) were significantly $(p<0.001)$ higher than those of pts without DVT (d $3: n=79, \bar{x}=623 \mathrm{ng} / \mathrm{ml}$; d 10: $n=79$, $\overline{\mathrm{x}}=586 \mathrm{ng} / \mathrm{ml}$ ). Taking a $\mathrm{D}$-Dimer concentration of $1000 \mathrm{ng} / \mathrm{ml}$ as cut-off point between pts with ( $1000 \mathrm{ng} / \mathrm{ml})$ or without DVT (<1000 $\mathrm{ng} / \mathrm{ml})$ the following PVS were observed: positive PVS on $d 3$ (d 10) : LMWH-group $81 \%(96 \%)$, UH-group $84 \%$ (96 \%); negative PVs on d 3 (d 10): LMWH-group $92 \%(97 \%)$, UFH-group $95 \%$ (100\%). These results convincingly demonstrate that determination of D-Dimer levels after hip arthroplasty can be used as a simple and reliable method for selection of pts with high risk of postoperative DVT.

Department of Internal Medicine, Section Hematology/Oncology, University of Münster, AlbertSchweitzer-str. 33, D-48129 Münster, FRG 
91

DOES THE RATIO OF PROTHROMBIN FRAGMENT F1+2 AND INR REFLECT THE ANTICOAGULANT EFFECT OF LOW INTENSITY PHENPROCOUMON? *K. -H. Zurborn und H. D. Bruhn

For evaluating prothrombin fragment $F 1+2$ as an indicator of the anticoagulant effect of cumarin this parameter was measured in 164 patients (mean age 63.3 years) on stable anticoagulation by phenprocoumon. These results were compared with those of healthy subjects without anticoagulation (mean age 54.6 years). $F 1+2$ plasma levels were significantly decreased by oral anticoagulation ( 0.45 vs 0.67 nmol/1). Even a low degree of anticoagulation (INR $<2.0$ ) reduced the $F 1+2$ value into the normal range $(0.32-1.2$ nmol/1). Changes in the plesma level of prothrombin fragment $F 1+2$ directly depended on the degree of oral anticoagulation. So this parameter and particularly the ratio between $F 1+2$ and INR seems to be suitable for the monitoring of the anticoagulant effect. This is especially true for low degree anticoagulation in which the effect cannot be satisfactorily measured by the thromboplastin time. This finding is of interest as in recent years low degree anticoagulation gained special interest in several clinical situations.

*Present address:

Klinik für Allgemeine Innere Medizin

I. Medizinische Klinik

Christian-Albrechts-Universität Kiel

Schittenhelmstraße 12

D-24105 Kiel, FRG

\section{3}

ASSOCIATION OF D-DIMER CONCENTRATIONS IN PLASMA OF CHD PATIENTS WITH THE SEVERITY OF ATHEROSCLEROSIS IN CEREBRAL, CORONARY AND PERIPHERAL ARTERIES

\section{J. Heinrich, H. Schulte, R. Schönfeld, E. Köhler, and G. Assmann}

Angiographic and post-mortem investigations have shown that the occlusion of a coronary artery by a thrombus is the precipitating event in the pathogenesis of acute myocardial infarction. The thrombus formation is localized on atherosclerotic lesions. Besides lipids, atherosclerotic plaques hold fibrin(ogen) split products. We tested whether a reduced endogenous fibrinolytic activity, leading to diminished concentration of fibrin split products, is linked to the occurence of coronary heart disease (CHD). Fibrinogen, prothrombin activation peptide $F 1+2$, plasminogen, PAI-1, d-dimers and C-reactive protein (CRP) were investigated in nearly 1,000 male patients of a coronary rehabilitation centre. Coronary angiography was performed and cerebral and peripheral arteries were investigated in detail. We found a strong association of d-dimers with the severity of CHD and cerebrovascular disease (CVD), as well as the presence of peripheral arterial disease (PAD):

\begin{tabular}{|c|c|c|c|c|c|c|c|c|}
\hline \multirow{4}{*}{$\begin{array}{c}\text { vessels } \\
\text { affected } \\
0\end{array}$} & \multicolumn{8}{|c|}{ D-DIMER (ng/ml) } \\
\hline & & CHD & & & CVD & & PAD & \\
\hline & mean & SD & $\mathrm{n}$ & mean & SD & $\mathbf{n}$ & mean SD & $\mathbf{n}$ \\
\hline & 265.2 & (212.8) & 26 & 378.5 & (254.6) & 300 & $\mathrm{PAD}+501.8(311.9)$ & 223 \\
\hline 1 & 360.7 & $(234.3)$ & $322 *$ & 417.0 & (270.5) & $209 *$ & PAD - $413.8(269.4)$ & 68 \\
\hline 2 & 421.7 & (263.9) & $305^{*}$ & 459.3 & $(296.4)$ & $202^{*}$ & & \\
\hline 3 & 559.2 & (319.5) & $259 *$ & 489.3 & (302.4) & $103 *$ & $(* ; p<0.0$ & \\
\hline
\end{tabular}

CRP showed a distinct, but not significant increase with increasing number of coronary stenoses $(0 / 1 / 2 / 3$ vessels affected: $1,7 \pm 2,8 \mathrm{mg} / 1 / 2,3 \pm 4,5 / 2,8 \pm 4,6$ $(3,5 \pm 5,6)$. All CRP mean values were below the pathological, inflammatory range of $>5 \mathrm{mg} / \mathrm{l}$. At present, it cannot be ascertained whether high concentrations of d-dimers directly result in atherosclerosis or whether they reflect an acute-phase reaction secondary to vessel disease.

Institute of Clinical Chemistry and Laboratory Medicine, Institute of Arteriosclerosis Research, both: University of Münster, D-48 129 Münster; LVA-Hospital Salzetal, D-32 066 Bad Salzuflen.

D-DIMER TEST DETECTS BOTH PLASMIN AND NEUTROPHIL ELASTASE DERIVED SPLIT PRODUCTS

R. Seitz, L. Lerch, F. Leugner, A. Immel, R. Egbring

Fibrin(ogen) split products containing two D-domains originate only from molecules cross-linked by factor XIIIa. Thus, high D-Dimer level is interpreted as result of ongoing coagulation leading to cross-linked fibrin and consecutive fibrinolysis. Besides plasmin, also human neutrophil elastase (HNE) can degrade fibrin(ogen), a so-called "alternative fibrinolysis pathway" (Plow, Blut 53:1;1986).

In this study, clots prepared by addition of $3 \mathrm{U}$ thrombin to $1 \mathrm{ml}$ purified human fibrinogen $(10 \mathrm{mg} / \mathrm{ml})$ were incubated in vitro with plasmin or HNE. In aliquots obtained from the supernatants, D-Dimer was assessed using a ELISA kit (Boehringer Mannheim). In 35 ulcerous colitis patients, D-Dimer, plasmin-antiplasmin complex (PAP, ELISA Behring) and HNE in complex with proteinase inhibitor (HNE-PI, IMAC, Merck) was measured.

Clots were dissolved not only by plasmin, and also by HNE dose dependently, after $24 \mathrm{~h}$ incubation with $40 \mu \mathrm{g} / \mathrm{ml}$ Elastase at $37^{\circ} \mathrm{C}$, a clot was macroscopically almost completely dissolved. In the supernatant, an increase of D-Dimer up to $1180 \mu \mathrm{g} / \mathrm{l}$ after $4 \mathrm{~h}$ was demonstrable. Thereafter, the D-Dimer decreased again, probably due to further digestion into smaller fragments. A statistical correlation of D-Dimer levels was found both with PAP ( $\mathrm{r}=0.467, \mathrm{p}=0.0062)$ and with HNE-PI complex $(r=0.509, p=0.0025)$ in 35 patients with ulcerous colitis.

This suggests that a elevated D-Dimer level might be caused not only by plasmin- but also by HNE-mediated fibrinolysis, particularly in patients with inflammatory disorders.

Philipps-University Hospitals, Dept. of Haematology, Baldinger Str., D-35033 Marburg, Germany. 
94

Fibrin(ogen) Derivatives in the Diagnosis of Disseminated ActivaTION OF COAGULATION AND FIBAINOLYSIS - EFFECTS OF IL-6 AND TNFa J.U. Wieding

A clinically relevant activation of coagulation leads to an increased fibrin generation. In DIC, for example, fibrinemia is characteristic. To gain insights into the dynamics and regulation of hemostasis activation, fibrin(ogen) derivatives and other markers of the hemostatic balance and activation as well as several cytokines were analyzed in courses of patients whose DHC was triggered by acute pancreatitis $(n=11)$, cardiopulmonary bypass surgery $(n=18)$ or acute septicemia induced by i.v. injection of 2 different bacterial lysates $(n=16$ courses of immunomodulatory treatment of malignant melanoma).

In these studies, markers of thrombin and fibrin generation (thrombin-antithrombin complexes, prothrombin fragments, soluble fibrin, fibrinopeptide A) correlated well with each other $(r>0.85)$. Time dependent courses correlated better with levels of interleukin-6 (IL-6) than tumor necrosis factor alpha (TNFa), IL-1, IL-2, IL-4, ELAM ICAM and, probably, IL-8. This finding agrees with recent results by v.d.Poll (1993) demonstrating that $\angle L-6$ activates coagulation. The acute-phase reaction, also mediated by $\mathrm{L}-\mathrm{G}$, started within $6 \mathrm{~h}$; time-triggered courses made the synthesis of C-reactive protein, fibrinogen, $a_{1}$-antitrypsin, ATIII etc. comparable.

In measurements of fibrinolysis activation, plasmin-antiplasmin complexes, fibrin(ogen) degradation products and (at the beginning of acute septicemia) plasminogen activator $(t P A)$ correlated well with each other $(r>0.85)$. Notably, in acute septicemia the onset of fibrinolysis obviously preceded the activation of coagulation: Lytic fibrin(ogen) splits increased more rapidly than markers of thrombin/fibrin generation. This hyperfibrinolysis immediately followed the increase in TNFa: it causes a tPA-release and, delayed by ca. 3 hours, the release of its inhibitor PAI-1, thereby subsequently repressing the initial hyperfibrinolysis.

Accordingly, in patients undergoing cardiopulmonary bypass surgery, TNFa-effects seemed to be less relevant than the IL-6 -mediated activation of coagulation. During acute pancreatitis, the trypsin activity, which probably leads to direct and indirect fibrinogen/fibrin turnover, correlated better with soluble fibrin and D-dimers than other parameters.

In conclusion, soluble fibrin and split products proved to be suitable parameters for monitoring of hemostasis activation. However, the intra- and inter-individual heterogeneity of fibrin(ogen) derivatives pose problems in comparing results from different methods; this applies especially to soluble fibrin, but also to D-dimers.

Universitätskliniken, D- $\mathbf{3 7 0 7 5}$ Göttingen, FRG

Evaluation of a Thromboplastin Reagent Based on Recombinant Tissue Factor in Patients with Various Haemostasis Disorders M. Barthels; C. Hohm, H. Poliwoda, H.J. Kolde, Med.Hochschule Hannover und Baxter Diagnostics, Unterschleissheim

The introduction of recombinant human tissue factor ( $\mathrm{r}-\mathrm{hTF}$ ) has opened the possibility to produce standardised thromboplastin reagents for clinical use without the technical problems associated with the handling of highly vascularized organs as raw materials. We have studied a commercial preparation of $\mathrm{r}-\mathrm{hTF}$ and synthetic phospholipids in comparison to a thromboplastin based upon a human placental extract (THP) in patients with various hemostasis disorders in the PT test and also in specific factor assays for factor VII and X on $\mathrm{KC} 10$ or Electra coagulometers respectively. Both reagents gave very similiar results in suspected healthy blood donors. In patients with heparin therapy $r-h$ TF was less influenced by heparin than THP due to the presence of a heparin neutralising compound in $\mathrm{r}-\mathrm{hTF}$. The difference in \%PT was up to $25 \%$. Similiar cases were seen also in cases with heparin and concomitant oral anticoagulation (OAC). In patients on OAC the PT values with both reagents correlated well in INR. The factor assays with both reagents gave very similiar results with a tendency towards slightly lower values with r-hTF at factor levels lower than $30 \%$. It was not investigated if this observation was related to the calibration of the assays. Results between $\mathrm{KC}$ and Electra agreed very well. The precision of r-hTF was excellent on both instruments. The different points of the calibration curve gave CV values of 0,7 to $3,6 \%$ (dilution $1: 1$ down to $1: 8, n=5$ days) on a $\mathrm{KC} 10$. The intra assay precision $(\mathrm{n}=10)$ was $1,4 \%$ for a pathological control on Electra. An inter assay CV of $2,3,1,0,3,4 \%(n=5)$ on normal or pathological controls respectively was found on a $\mathrm{KC} 10$. (CV calculations in sec.)

In conclusion our study shows that $\mathrm{r}-\mathrm{hTF}$ is a thromboplastin which detects defiencies of the extrinsic coagulation at least as sensitive as HPT and is not influenced by heparin even at relatively high dosage.
CLINICAL EVALUATION OF A CAPILLARY BLOOD METHOD FOR PROTHROMBIN TIME USING A RECOMBINANT TISSUE FACTOR BASED THROMBOPLASTIN REAGENT

Seyfert U.T. Abt.Klinische Hämostaseologie, Universitätsklinik Homburg/Saar

For ambulant patients on oral anticoagulant therapy the performance of the prothrombin time (PT) on capillary blood (CB) has certain advantages in some hospitals. We have ev aluated a new $C B$ procedure $(1)$ for the PT using a PT reagent based on recombinant humian tissue factor and synthetic phospholipids (Innovin "n "I", Baxter Diagnostics) in which the capillary blood is drawn into a special citrate solution. The $\mathrm{PT}$ on $\mathrm{CB}$ was performed on a $\mathrm{KC} 10$ coagulometer in comparison to the plasma method with "I" and the Hepatoquick ${ }^{\circ}$ (HO, Boehringer Mannheim). The CBPT with "I" does not require a preincubation step and thus the total assay is reduced. The ISI value for the $C B$ method with " $I^{n}$ was obtained by analyzing 21 patients on stable oral anticoagulation and 11 normals. The log transformed clotting times of the CB-PT were compared either against the plasma method with "I" (P-PT) or the HO method using the ISI values that are assigned for the plasma procedure of " 0 " or the HO respectively. ISI values of 0.74 and 0.73 for the CP-PT were obtained using the two reference methods respectively. Method comparisons of the three methods in INR showed the following equations:

1. CB-PT versus P-PT with I: $\quad y=0.978 x+0.057, \quad r=0.946$

2. CB-PT with I versus $\mathrm{HQ:} \quad \mathrm{y}=1.038 \mathrm{x}-0.002, \quad \mathrm{r}=0.965$

3. HQ versus P-PT with I: $\quad y=0.951 x+0.066, \quad r=0.969$

The same data were also analyzed in PT-\% in which the CB-PT with "I" was calibrated with a three calibrator set with normal fibrinogen concentration in a dilution equivalent to the $\mathrm{CB}$ dilution, assuming a normal hematocrit. The regression lines for the same sequence of method comparisons had the following parameters:

1. CB-PT versus P-PT with I: $\quad y=0.984 x+2.952, \quad r=0.982$

2. CB-PT with I versus HQ: $\quad y=1.078 x+2.484, \quad r=0.982$

3. HQ versus P-PT with $\mathrm{I}: \quad \mathrm{y}=1.000 \mathrm{x}-0.300, \quad \mathrm{r}=0.958$

These data show that a reliable determination of a capillary blood PT with a reagent based on recombinant tissue factor is possible leading to considerable savings in time and costs.

$1_{\text {(H..J. Kolde, B. Denzler, pers. communication) }}$

Address: Uniklinik d. Saarlandes, Klin. Hämostaseologie, Herrn Dr. Seyfert, Haus 75, 66421 Homburg/Saar

97

EVALUATION OF DIFFERENT PT-METHODS USING RECOMBINANT TISSUE FACTOR

G. Mustafa, S. Kapiotis, P. Quehenberger, W. Speiser

Beside monitoring of patients receiving oral anticoagulant therapy, screening of coagulation activity in preoperative and parenteral nourished patients are main issues of PT testing. Since conventional thromboplastins are difficult to standardise and are not devoid of risks of transmitting viral disease, being extracted from human or animal tissues, we compared in our report three conventionally methods (PT 1: Thromborel S, Behringwerke AG; PT2: Normotest; Nycomed Pharma AS; PT3 Immunoplastin, Immuno AG) with two tests (PT4 Innovin, Baxter; PT5 Recombiplastin, Ortho Diagnostic System; ) employing recombinant thromboplastin. 103 patients not treated with anticoagulant therapy were tested for single factor activity (FII, FV, FVII, FX) to determine the sensitivity (SN) and specificity (SP) of the PT-tests in detecting single factor deficiencies. The cut off level of PT test systems was $75 \%$. According to ROC-curves PTs showed a SN of $52 \%$ to $69 \%$ at a SP level of $89 \%$ to $97 \%$ (PT1 SN: $53 \%$ SP: $97 \%$; PT2 SN: $69 \%$ SP:89\%; PT3 SN: 67\% SP 92\%; PT4 SN: 63\% SP:96\%; PT5 SN: 52\% SP: 95\%).

Sensitivity of PT reagents in detecting reduced FVI activity:
\begin{tabular}{|ccccc|cccc|}
\hline PT1 & PT2 & PT3 & PT4 & PT5 & FII & FV & FVI & FX \\
\hline 59 & 46 & 60 & 59 & 58 & 135 & 111 & 36 & 93 \\
85 & 67 & 76 & 77 & 72 & 102 & 114 & 32 & 79 \\
81 & 73 & 85 & 85 & 83 & 99 & 157 & 54 & 99 \\
65 & 55 & 67 & 68 & 68 & 106 & 74 & 59 & 82 \\
97 & 65 & 74 & 89 & 96 & 110 & 142 & 61 & 76 \\
74 & 47 & 73 & 54 & 56 & 81 & 113 & 40 & 103 \\
88 & 90 & 82 & 79 & 81 & 100 & 97 & 57 & 114 \\
68 & 60 & 71 & 59 & 63 & 116 & 118 & 52 & 96 \\
86 & 52 & 47 & 57 & 79 & 92 & 103 & 54 & 79 \\
77 & 62 & 73 & 78 & 82 & 72 & 110 & 68 & 72 \\
72 & 47 & 64 & 55 & 73 & 120 & 88 & 44 & 96 \\
\hline $5 / 11$ & $10 / 11$ & $8 / 11$ & $6 / 11$ & $5 / 11$ \\
correct detected FVII deficiencies / number of patients & & \\
\hline
\end{tabular}

Clinical Institut for Medical and Chemical Lab Diagnostics

Währingergïrtel 18-20, A-1090 Wien, AUSTRIA 
LEVELS OF ANTIGENIC AND FUNCTIONAL FIBRINOGEN IN HOSPITALIZED PATIENTS WITH RESPECT TO ACUTE PHASE RESPONSE

B. Krammer, O. Anders. H.-R. Nagel ${ }^{\#}$, C. Burstein\#, B. Ernst\#, M. Steiner\#

The diagnosis dysfibrinogenaemia requires discordance between functional and antigenic fibrinogen. However, the level of discordance is not yet established. Furthermore, different assays for functional fibrinogen are currently being used. Therefore we have investigated 79 consecutive hospitalized patients using three different methods for determination of fibrinogen: Clauss fibrinogen (Fbg-C, Multifibren Behring, KC 10), derived fibrinogen (Fbg-D, ACL 3000, IL, PTFibrinogen-HS) and immunological fibrinogen (Fbg-l, BN 100, Behring). C-reactive protein (CRP) was included to monitor acutephase response. The highest values (mean \pm SD) were found for Fbg-D $(4.62 \pm 1.94 \mathrm{~g} / \mathrm{l})$ followed by Fbg-C $(4.10 \pm 1.91 \mathrm{~g} /)$ and Fbg-l $(3.46 \pm 1.46 \mathrm{~g} / \mathrm{l})$. Significant correlations were calculated between all three methods (Fbg-D:Fbg-C $r=0.92$, Fbg-D:Fbg-1 $r=0.97$, Fbg-C.Fbg-1 $r=0.89$ ). Fibrinogen demonstrated good correlation to CRP irrespective of the method used $(r=0.73-0.75)$. However, the mean ratio $\mathrm{Fbg}-\mathrm{C}$ to $\mathrm{Fbg}-1(1.188 \pm 0.238)$ was significantly different from the ratio Fbg-D to Fbg-l $(1.346 \pm 0.156)$. The lower limit (mean - 2 SD) would be 0.712 for the ratio Fbg$\mathrm{C}: \mathrm{Fbg}-\mathrm{I}$ whereas 1.034 for the ratio Fbg-D:Fbg-l. These differences should be considered when cases of suspected dysfibrinogenaemia are to be evaluated. However, acute-phase reaction can apparently be neglected for this diagnostic procedure.

University of Rostock, Department of Internal Medicine, Institute of Clinical Chemistry \& Pathobiochemistry ${ }^{*}$, Heydemann-Str. 6, 18055 Rostock, Germany

99

\section{LUPUS ANTIKOAGULANTS \\ SENSITIVITY OF DIFFERENT LABORATORY TESTS AND PRAEVALENCE IN 759 PATIENTENS \\ S. Ehrenforth, S. Siegert, I. Scharrer}

Introduction: The identification of antiphospholipid antibodies (APA), especially of lupus anticoagulants (LA) has received increased attention due to their association with different clinical complications. However, the laboratory diagnosis, particularly of weak LA continues to present many problems. As yet there is still no single, reliable test for LA, and thus scveral test methods have to be used concurrently.

The aims of the present study were 1. to evaluate the praevalence of LA in young pts. with thromboembolism or autoimmune disease, recurrent spontaneous abortions, thrombocytopenia or cerebral ischaemia; 2 . to validate the results of new test methods for LA and 3. to compare the LA sensitivity of different laboratory methods.

Patients and methods: At present our LA screening include following tests: APTT, APTT mixing studies, diluted Russels vipper venome time, kaolin clotting time and ELISA testing for anti-cardiolipin antibodies (aCL). As confirmatory methods we are performing the Staclote test as a platelet neutralization procedure and the recently introduced Textarin test. The circulating anticoagulant activity (ICA) is measured for each LA positive patient. Hepzyme is employed to correct the heparin effect.The study group consisted of 759 pts. (416 woman, 343 men) aged 12 and 78 (median 40.1 ys). 80 healthy persons served as controls. Results: 90759 pts. fulfilled the criteria for positive LA status (at least two abnormal clotting times plus clinical signs of the above diseases), according to a praevalence of $11.8 \%$. In these 90 pts. the KCT was positive in $81.1 \%$, the dRVVT in $82.7 \%$, APTT mixing studies in $82.9 \%$, the Staclot in $75 \%$ and the ICA in 74.2\%. Only the Textarin test (18 Units) was positive in nearly all pts. proven to be LA positive $(96.3 \%$ sensitivity). aCL antibodies could be determined in $44 / 90$ (48.8\%) of confirmed LA positive pts. (with 5 in the IgG fraction only, 11 in the IgM fraction only and with 28 positive for both IgG and IgM antibodies).

Conclusion: The above results show that the complete range of laboratory tests are required to confirm the presence or absence of $\mathrm{LA}$.
DETERMINATION F.VIII INHIBITORS (BETHESDA-METHOD): CLOTTING VERSUS CHROMOGENIC.

B. Moritz ${ }^{1}$, H. Langl, I. Scharrer ${ }^{2}$, W. Schramm ${ }^{3}$

For determining the presence and the amount of an F.VIII inhibitor, most frequently the Bethesda method is used. Equal volumes of normal pooled plasma and of diluted patient plasma are incubated for 2 hours at $37^{\circ} \mathrm{C}$. The residual F.VIII activity is assayed and and the inhibitor units are calculated.

The accuracy of this method is affected by several parameters: (1) since inhibition is a time-dependent process, incubation has to be strictly observed; (2) sometimes, inhibitors don't have a simple, linear reaction kinetics; (3) the accuracy of the F.VIII determination reflects on the former result.

The F.VIII residual activity is usually measured until now with one-stage clotting assays. This study was undertaken to show whether the chromogenic assay (Immunochrom ${ }^{\otimes}$ F.VIII:C) can also be used.

Samples in the range between 0.8 and 40 B.U. were tested. Both methods, the clotting and the chromogenic, correlate very well $(r>0.999)$, but the chromogenic methods tends to give lower F. VIII inhibitor levels. In the dayto-day-reproducibility, the CV(\%) range from $1: 4$ to 15.3 for the clotting method, and 4.5 to 8.5 for the chromogenic method. Especially at higher levels of F. VIII inhibitor, the clotting method gives higher CV(\%)'s.

Samples were routinely tested for residual F.VIII activity right after the 2 hour incubation. Alternatively, the incubated plasma samples can be kept on ice for 2 hours before measuring the F.VIII residual activity (performed only for the chromogenic method).

We conclude that the advantages of the chromogenic F.VIII determination easy performance and automatisation and better reproducibilty - can be used also for measuring F.VIII inhibitor levels using the Bethesda-Method.

(1) Immuno AG, Industriestr. 67, A-1220 Vienna / (2) Universitätskliniken, Zentrum der Inneren Medizin, Theodor-Stern-Kai 7, D-60596 Frankfurt, (3) Med. Klinik Innenstadt, Hämostaseologische Abteilung, Ziemssenstr. 1, D-80336 München 


\section{IMMUNE STATUS OF HIV- AND HCV- NEGATIVE PAEDIATRIC PATIENTS AFTER LONGTERM TREATMENT WITH INTERMEDIATE FACTOR VIII CONCENTRATES S. Ehrenforth*, M. Funk, D. Mentzer, R. Linde, W. Kreuz}

Different studies published recently suggest that treatment of HIV-infected haemophiliacs with high-purity FVIII concentrates may preserve immune function in contrast to treatement with intermediate-purity FVIIl concentrates. But as yet, there is no published study performed exclusively on HCV-and HIV-negative pts. treated with IP-FVIII concentrates, although the influence of both infections on the immune system is well known. In our follow-up (1983-1993) 96 children (77 haemophiliacs, 19 with von Wiilebrand disease) were monitored as follows: IgG, M, A; lymphocyte subsets (CD3, 19,4,8,57, HLA-DRt); lymphocyte proliferative response (CD3, phytohaemagglutinin, pokewced, concavalinA); response to intradermally-injected recall antigens (Multitest, Bio-Merieux). Haemophiliacs received either Humate $P$ P (Behring, Germany) or FVIII STIM IIIB (Immuno, Germany) (36 prophylactically, 41 on demand). Children with vWD were treated on demand with HumatePB. The mean counts of CD 3, 19,4,8 cells and CD4/8 ratio showed an age-depending decrease. In contrast the mean values of CD57 gradually increased during the follow-up. None of these changes were significantly different to those of healthy controls. HLA-DR+ cell counts were stable in all patients. The lymphocyte proliferative response or Ig levels revealed no significant differences when companing patients with age-related healthy controls. However, a small age-dependent rise of $\mathrm{Ig}$ levels was observed over the follow-up period. Skin tests showed no anergy in any patient. An influence of treatment intensity on the patients immune parameters could not be demonstrated. In conlusion our results, from the longest and largest such follow-up reported in $\mathrm{HCV}$ - and HIV-negative haemophiliacs, demonstrate stability of different immune parameters indicating that even long-term exposure to IP-FVIII concentrates may not compromise the immune response.

Dept. of Paed. and *Internal Med., Univ. Hospital, Frankfurt, Germany

102

\section{THE NATURAI COURSE OF HCV-INTECTION IN HIV-SERO NEGATTVE} AND HIV-SEROPOSITTVE HEMOPHIILIACS

\section{M. v. Depka Prondzinski, 1. Schamer, *B. Weber}

Objective: To describe the natural history of Hepatitis $C$ infection and its clinical outcome in multitransfused hemophiliacs with HIV-coinfection. Patients and Methods: 149 patients with congenital Hemophilia A or B were evaluated retrospectively over the past 13 years. Liver enzymes and surrogate markers of HIV-infection (p24, CD4, CD8 counts) were tested and HBV, HBSAG, HCV, HCV-CDNR-PCR and HIV-1/2 assays performed. Patients were divided into three subgroups (group A: HIVand HCV-PCR-negative patients, group B: HIV-seronegative and HCVPCR-positive patients, group C: HIV-and HCV-PCR-positive patients), to point out differences between the subgroups as to the clinical outcome of HCV-infection. Results: Epidemiological data on HCV and HIV coinfections are listed in Tab1. We found significant differences of ALT concentration between group $A$ and $B$, but not between group $A$ and $C$ neither regarding liver enzymes nor clinical progression of liver disease.

\begin{tabular}{|l|c|l|l|}
\hline Tab1 & \multicolumn{4}{|c|}{ HCV-HIV coinfection (n=121) } \\
\hline HCV neg / HIV neg & $31(26 \%)$ & HCV pos / HIV neg & $61(50 \%)$ \\
\hline HCV neg / HIV pos & $0(0 \%)$ & HCV pos / HIV pos & $29(24 \%)$ \\
\hline \multicolumn{4}{|c|}{ HCV-PCR tested patients (n=46) } \\
\hline PCR neg / HIV neg & $10(22 \%)$ & PCR pos / HIV neg & $19(41 \%)$ \\
\hline PCR neg / HIV pos & $2(4 \%)$ & PCR pos / HIV pos & $15(33 \%)$ \\
\hline
\end{tabular}

Conclusion: Multitransfused hemophiliacs are mostly HCV infected. HCV-DNA-PCR measurements demonstrate high prevalence of HCV-DNA particularly in HIV-coinfected patients. The impact of HIV infection on the course of chronic hepatitis $C$ is not yet clear. Our data suggest, that HCV-associated liver disease in HIV coinfected patients may be associated with long-term clinical and laboratory stability.

Dept of Angiologie, "Dept of Medical Virology, University Hospital Frankfurt, Theodor-Stern-Kai 7, 60590 Frankfurt am Main, FRG
CLINICAL TRIALS PERFORMED WITH AN AIDS CANOIDATE VACCINE Josef W. Mannhalter and *Martha M. E ibl Immuno AG, Vienna, Austria, and *Institute of Immunology, University of Vienna, Vienna, Austria

The safety and immunogenicity of an AIDS candidate vaccine (based on the use of the recombinant HIV-1 envelope glycoprote in 160) was tested in over 100 HIV-1-seronegative and over $250 \mathrm{HIV}-1$-seropositive volunteers. The studies marked "NIAID" were performed by the National Institute of Allergy and Infectious Diseases' AIDS vaccine evaluation group. The results of these studies are briefly summarized below.

\begin{tabular}{|c|c|c|c|c|c|c|c|}
\hline \multicolumn{3}{|c|}{ 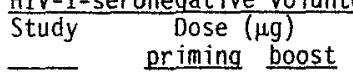 } & t & T cell & CTL & $\begin{array}{l}\text { Binding } \\
\text { antibody }\end{array}$ & $\begin{array}{l}\text { Neutr. } \\
\text { antibody }\end{array}$ \\
\hline NIAI00041) & $\begin{array}{c}12.5 \\
50 \\
50\end{array}$ & $\begin{array}{l}12.5 \\
50 \\
200\end{array}$ & & $\begin{array}{c}t+++ \\
+++t \\
+++\end{array}$ & $\begin{array}{l}- \\
+\end{array}$ & $\begin{array}{l}+ \\
++ \\
++\end{array}$ & $\underset{+}{ \pm}+$ \\
\hline$\frac{\text { HIV-1-sero }}{\text { Study }}$ & $\begin{array}{l}\text { opositiv } \\
\text { CD4 D } \\
\text { ounts pr }\end{array}$ & $\begin{array}{l}\text { e volur } \\
\text { ose }(\mu) \\
\text { iming I }\end{array}$ & $\begin{array}{l}\text { Inteer } \\
\text { boost } \\
\text { boos }\end{array}$ & $\begin{array}{r}\mathrm{T} \text { ce } \\
\mathrm{memo} \\
\end{array}$ & CTL & $\begin{array}{r}\text { Binding } \\
\text { antibody }\end{array}$ & $\begin{array}{l}\text { Neutr. } \\
\text { antibody }\end{array}$ \\
\hline $\begin{array}{l}\text { NIAID1012) } \\
\text { EUROP II } \\
\text { EUROP II } 2\end{array}$ & $\begin{array}{l}\geq 600 \\
\geq 500 \\
200-500\end{array}$ & $\begin{array}{r}50 \\
100 \\
100\end{array}$ & $\begin{array}{r}50 \\
100 \\
100\end{array}$ & $\begin{array}{c}+++ \\
\star \\
*\end{array}$ & ++ & $\begin{array}{l}* \\
x \\
x\end{array}$ & $\begin{array}{l}* \\
x \\
x\end{array}$ \\
\hline
\end{tabular}

* tests ongoing; $X$ will be tested.

In both HIV-1-seronegative and HIV-1-seropositive volunteers the candidate vaccine proved to be safe and immunogenic. Side effects were judged tolerable by the investigators and the vaccinees. Depending on the vaccine dose given and the immunization regimen applied, cell-mediated ( $T$ cell memory, CTL) and humoral (including.neutralizing antibodies) immunity could be induced.

1) R.B. Belshe et al., J.Inf.Dis, in press; 2) D.H. Schwartz et al. IXth International Conference on AIDS, Berlin 1993 
A High Purity Factor VIII Concentrate with an Increased Safety Margin - Pharmakokinetics and First Data of Clinical Efficacy and Safety

R. Zinmermann, P. Lages, A. Huth-Kühne, Rehabilitation Hospital and Hemophilia Center, 69123 Heidelberg

In a previously published multicenter clinical trial the pharmakokinetics of a new high purity factor VIII concentrate were evaluated in comparison with those of the predecessor factor VIII concentrate. The trial was conducted and analyzed according to the recommendations of the Factor VIII/Factor IX Scientific and Standardization Committee of the ISTH. The evaluation of 16 study patients with severe hemophilia A showed no significant differences between both preparations. Thus demonstrating that further steps of virus inactivation did not affect the biologic capacity. The factor VIII concentrate Immunate STIM plus is a high purity factor VIII:C/VWF complex concentrate obtained from plasma by ion-exchange chromatography. The manufacture includes two independent virus inactivation procedures with vapor heating and an intensive treatment with polysorbat, as well as chromatographic vjcus removal with an overall HIV reduction potential of $>1014$.

31 males with hemophilia $A$ were treated with the new product, all of them with severe hemophilia A. 8 of them received prophylactic treatment, 23 were treated on demand, 1 during surgical procedures and 1 patient for inducing immunotolerance because of an factor VIII inhibitor. Prophylactic treatment was given at a dosage from 14 .

33 I.U. per $\mathrm{kg}$ body weight. The observation period was 1 15 months with a mean of 8.4 months. The individual in vivo recovery and half-life were calculated in regular intervals.

A17 patients tolerated the new native high purity factor VIII concentrate very well. There was no documented adverse reaction. No virus infection and no factor VIII inhibitor developd. The clinical efficacy of the preparation was excellent.

105 INACTIVATION AND PARTITIONING OF HIV-1 AND MODEL VIRUSES
DURING THE MANUFACTURE OF IMMUNINE ${ }^{\circledR}$ AND IMMUNATE N. Barrett, G. Pölsler, J. Eibt, F. Domer

Blood products derived from human plasma are manufactured on an industrial scale from plasma pools consisting of some thousands of single donor units. Blood borne viruses may potentially occur in such plasma pools despite extensive screening of each single donor plasma unit for different viral markers. Therefore, every manufacturing procedure for any specific blood product must be designed to include sufficient viral removal steps and have inactivation capacity

for a wide range of viruses. Immunine ${ }^{(m)}$, respectively, were introduced in 1991. Two separate virus Immunine ${ }^{8}$, respectively, were introduced in 1991. Two separate virus
inactivation procedures are applied, one at the beginning and the other at the end of the manufacturing process. The first is a Polyglycate TM treatment developed by immuno. This has been shown to be highly efficacious at inactivating retroviruses. The second is vapor heating, which involves heating of a moistened powder under pressure in a closed container in a protective gas atmosphere. This procedure has been demonstrated in long term clinical trials to render products safe with respect to virus transmission. The virus inactivation capacity of these procedures and the virus partitioning capacity of the manufacturing processes for Immunate ${ }^{(2)}$ and Immunine ${ }^{(2)}$ has been validated according to EC/CPMP guidelines 1 in preclinical studies. These studies have demonstrated an overall reduction factor (ORF) for Immunate of $\geq 14.7$ for HIV-1, $\geq 18.4$ for Tick-Bome Encephalitis Virus (TBEV), 11.3 for Pseudorabies Virus (PRV) and $\geq 9.7$ for Equine Rhinovirus (ERV). The following ORF's for Immunine were obtained: $\geq 22.9$ for HIV $-1,>27.8$ for TBEV, $\geq 18.3$ for PRV and $\geq 14.5$ for ERV. These data suggest that the incorporation of two separate virus inactivation procedures in the manufacturing processes used constitutes an aggressive approach for removing and inactivating potential viral contaminants.

1 Commission of the European Communities (1991). Ad Hoc Working Party on Biotechnology/Pharmacy - Note for Guidance, Vatidation of Virus Removal and inactivation Procedures. Iil/8115/89-EN
DOUBLE VIRUS INACTIVATED F VIII CONCENTRATE

VIRLS VALIDATION AND IMMUNOLOGICAL ASSESSMENT OF F VIII SDH R. Kotitschkel, H. Dichtelmullierl, D. Rudnickl, J. Holzapfell, M. Kloft 5. Elödil, I. Zimonyi2 and A. Marosi2

F VIII SOH (SDH $=$ solvent detergent and dry heat $=100{ }^{\circ} \mathrm{C}, 30 \mathrm{~min}$ ) from Biotest Pharma is a high purity (specific activity $=2-100$ ) F VIII concentrate manufactured from large human plasma pools. Heat treatment in the final container of the freeze dried product causes a loss of about $15 \%$ of the F VIII activity at a residual moisture content of about one per cent (range 0.5 to 1.5 per cent). Virus validation studies have shown virus inactivation/reduction rates during the $F$ VIII manufacturing process for coated viruses such as: HIV-1 $>14.1 \log 10 ;$ PSR $>18.0 \mathrm{log} 10$; VSV $>13.9$ $\log 10 ;$ BVDV $>11.4 \log 10 ; \mathrm{HCV}>4.5 * \log 10$ and non enveloped viruses such es: Parvo $=3.7 \log 10 ;$ Reo $>5.3 * * \log 10$ and HAV $>13.9 \log 10$. The total protein for $1000 \mathrm{IE} \mathrm{F} \mathrm{VIIIC} \mathrm{in} 10 \mathrm{ml}$ is approximately $10 \mathrm{mg}$, of which $0.12 \mathrm{mg}$ is $F$ VIIt and $6 \mathrm{mg}$ uhf; i.e. more than $60 \%$ of the total protein is pure $F$ VIII/vif complex. The integrity of the F VIII molecule in F VIII SDH was shown by comparing the unheated and heated F VIII preparation. "In vitro" techniques were procoagulant activity assays, sodium dodecyi sulfate-polyacrylamide gel electrophoresis (SDS-PACE) and molecular size distribution chromatography (FPLC). Epitope mepping was performed on rabbit antisera using the Ouchterlony technique, two dimensional immunoelectrophoresis and Western blot, using the immunoassay protein-blot with the dot-blot microfiltration. The immunological tests did not reveal the presence of new antibodies against F VIII, indicating that the double virus inactivation does not induce the development of neoantigens. Folerability and efficacy of F VIII SDH was shown in clinical studies on hemophilia A patients.

Conclusion: SD-treated F VIII has been submitted to a second virus inactivation procedure (dry heat treatment), without causing significant biochemical or immunological changes, for further increasing the virus safety of the product.

* TNBP/Tween only * heat treatment only

1) Biotest Pharma GmbH, Dreieich

2) Dept. of Haematology of Hein Pál Children Hospital, Budapest
107

COMPARISON OF FACTOA IX CONCENTRATE AND PROTHROMBIN COMPLEX CONCENTRATE WITH REGARD TO THEIR THROMBOGENIC POTENTIAL

\section{H.P. Klöcking}

A number of occurrences of thrombotic episodes and disseminated intravascular coagulation asociated with the use of prothrombin complex concentrates have been described. To avoid this problem purer factor IX concentrates with very littie or no factor II, VII and $X$ were introduced for therapeutic purposes.

The objective of this study was to compare two formulations of factor IX - a purified factor IX concentrate (Octanyne ${ }^{\mathcal{F}}$, OCTAPHARMA, Vienna) and a prothrombin complex concentrate (PPSB, Prothrombin complex human, OCTAPHARMA, Vienna) with regard to their thrombogenic potential in vitro and in vivo. For both preparations the in vitro tests for thrombogenicity showed no evidence of the presence of activated clotting factors. Fibrinogen clotting times were $>6 \mathrm{~h}$ at $37^{\circ} \mathrm{C}$. The thrombin generation time TGT 50) was greater than 10 minutes. Non-activated partial thromboplastin times at 1/10 dilution were in the range of $313-417 \mathrm{sec}$. (control $268 \mathrm{sec}$.) The in vivo thrombogenicity of the concentrates has been evaluated in the venous stasis model acc. to WESSLER in rats. A total of 30 rats were tested with 3 batches of factor IX concentrate as currently formulated $(0.11 . \mathrm{U}$. heparin $/ 1$ 1.U. FIX). The mean score of thrombus formation ranged from $0-0.2$ atter application of $400 \mathrm{l.U}$. FIX/kg. Two batches of the prothrombin complex concentrate (0.4 I.U. heparin/1I.U. FIX) were tested up to $300 \mathrm{I.U}$. FIX/kg of each. The mean score ranged from $0.7-1.0$. The generally accepted upper limit is a mean score of 2.0 . Below this value, preparations are regarded as low- or non-thrombogenic.

Thus both concentrates of factor IX showed no thrombogenic potential in the stasis model according to WESSLER in rats and in the in vitro tests of thrombogenicity.

Institute of Pharmacology and Toxicology, Medical School Erfurt, Nordhäuse Str. 74, D-99089 Erfurt 
FEASIBILITY OF DOUBLE VIRUS INACTIVATED FACTOR VIII AND IX CONCENTRATES FOR PERIOPERATTVE CONTINUOUS INFUSION Schoppmann A., Weber A., Schönhofer W., Hondl F., Linnau Y., IMMUNO AG Vienna

In the treatment of hemophilia patients, the availability of high purity factor concentrates has recently encouraged clinicians to use perioperative continuous infusion of F VIII or F IX in order to prevent or reduce bleeding. In contrast to previous repeated high dose bolus injections, this treatment regimen allows for a constant activity stightly above the minimum coagulation factor level necessary to maintain hemostasis. The total cost of treatment can be reduced and possible side effects prevented like those previously reported after large doses of F DX complex. The new application form, however, requires stable products which tolerate slow passage through the infusion device. Our objective was to test in vitro the F VIII concentrate RMMUNATE $^{\otimes}$ (STIM plus) and the F $\mathrm{XX}$ concentrate MMUNINE (STTM plus) under conditions of long-term contact to the tubing device and storage container of an automatized infusion pump (Cadd-1 ${ }^{\circ}$, Pharmacia Deltec Inc., USA) at room temperature. Infusion rates were chosen to mimic the clinical situation. Control samples were not infused through the pump but otherwise treated identically. Test samples were drawn before and at $1,3,5,8,24$ and 48 hours after the onset of each infusion run. F VIII:C activity was determined both by chromogenic and 1-stage assays. In addition the 2-stage assay was performed for indication of activated $\mathrm{F}$ VIII. F IX:C activity was determined by aPTT, activated factors were measured by NAPTT and mixture of chromogenic substrates specific for $F$ Ila, $F X_{a}$, plasmin and prekalikrein activator (PKA) activities. The results show equivalent data between test samples and controls with no loss of activity of F VIII or F DX inside the infusion system. The potencies both of IMMUNATE ${ }^{\circledR}$ and IMMUNINE ${ }^{\oplus}$ remained within $100 \pm 20 \%$ of labelled values within 48 hours after onset of infusion. In conclusion two high purity coagulation factor concentrates have been shown to be useful for continuous infusion when using the automatic device tested and within the test criteria applied. Clinical experience consistent with these data bave been previously reported for IMMUNINE

*Present address: IMMUNO AG, Industriestr. 72, A-1220 Vienna, Austria
110

VIRUS-INACTIVATION OF FRESH FROZEN PLASMA BY DIFFERENT METHODS: EFFECT ON THE IN VITRO COAGULATION CAPABILITY

H. Riess, T. Zeiler, G. Hintz, R. Zimmermann, C. Müller and D. Huhn

Fresh frozen plasma (FFP) remains the main therapeutic option in the treatment of complex disorders of hemostasis, such as DIC or hyperfibrinolysis. To diminish the risk of virus transmission virucidal treatment of FFP has been recommended.

To determine the effects of methylenblue phototreatment (MBP) or the solvent/detergent method (SD) on hemostatic parameters in fresh frozen plasma we devided 11 double-sized plasmapheresis plasmen in pairs of 250 ml. One group was submitted to MBP (MBP-plasma). The other group was treated according to $\mathrm{AABB}$ standards (controls:C-plasma). These two groups were compared with another group of ten units of SD-plasma from different charges. Laboratory tests were done on samples taken from the simultaneously thawed FFPs.

Main results (mean $\pm S D$ ) are given in the table:

\begin{tabular}{llll} 
parameter & C-plasma & MBP-plasma & SD-plasma \\
PT (s) & $11.31 \pm 0.89$ & $12.27 \pm 0.66$ & $11.8 \pm 0.24$ \\
aPTT (s) & $34.5 \pm 4.4$ & $39.8 \pm 3.8^{*}$ & $35.8 \pm 4.72$ \\
Fibrinogen (g/l) & $2.77 \pm 0.72$ & $1.80 \pm 0.53 *$ & $2.32 \pm 0.05$ \\
F V (\%) & $94.5 \pm 23.7$ & $73.4 \pm 14.8^{*}$ & $59.5 \pm 3.27 *$ \\
F VIII (\%) & $86.7 \pm 25.3$ & $58.1 \pm 19.6^{*}$ & $64.7 \pm 11.3^{*}$ \\
plasminogen (\%) & $98.6 \pm 14.1$ & $97.8 \pm 21.8$ & $98.7 \pm 2.21$ \\
antiplasmin (\%) & $100.9 \pm 11.5$ & $96.0 \pm 14.4$ & $20.9 \pm 3.78 *$ \\
protein S (\%) & $110.5 \pm 20.2$ & $110.5 \pm 15.6$ & $62.0 \pm 7.87^{*}$ \\
C1-inhibitor & $107.6 \pm 19.2$ & $102.5 \pm 14.7$ & $76.3+2.67^{*}$ \\
\multicolumn{4}{c}{ * significant differences as compared with C-plasma }
\end{tabular}

We conclude that virus inactivation using these methods clearly impairs the quality of fresh frozen plasma in terms of coagulation and thus may reduce the therapeutic benefit.

Universitätskdinkum Rudolf Virchow, Department of Internal Medicine, Spandauer Damm 130, 14050 Berlin, Germany.
Safety Considerations for the Production of Recombinant Factor VIII from Mammalian Cell Culture

Berthold G. D. Bödeker

The production of the blood clotting Factor VIII by recombinant technology using Baby Hamster Kidney cells yields a product - Kogenate TM - of high quality with improved safety compared to plasma derived Factor VIII preparations. The safety related features of the product and the production process will be discussed. Theoretical safety issues for recombinant Factor VIII (IFVIII) could come from infectious agents from the cell line, biological components such as bovine insulin, human albumin and human transferrin from the culture medium as well as host cell impurities in the final product. Extensive testing of the cell line showed that the producing cells are free from any infectious viruses or retroviruses and microbial contaminations. In addition, each fermentation campaign is tested for the absence of adventitious viruses at the end of the run. Raw materials from human origin are pasteurized. Bovine insulin is obtained from BSE-free countries, tested for the absence of viruses and virus-inactivated. An additional virus safety feature is included in the purification process, which reduces virus titers by 6 to 12 logs depending on the model virus. This is achieved by a combination of vinus inactivation using detergent and clearance through several column chromatography steps. The purification process also efficiently reduces host cell impurities. DNA content in the final product is below $10 \mathrm{pg} / 1000 \mathrm{IU}$, hamster cell protein below $10 \mathrm{ng} / 1000 \mathrm{NU}$. Overall, clinical use for over 5 years and market experience for almost 1 year in the USA clearly prove that the rFVIII product Kogenate ${ }^{T M}$ is not only efficacious and of high quality, but also safe. 
FLOW CYTOMETRY ANALYSIS OF THE PHARMACOKIVETICS OF LMM-HEPARIN USING A FLUORESCENTLABELED DERIVATTVE

L. Piazolo, J. Harenberg, R. Malsch and D.L. Heene

A new preparation of low molecular mass heparin (LMMH) was used to investigate the pharmacokinetic profile of LMM-heparin. Fluorescein-5-isothiocyanate (Fitc) has been bound by "endpointattachment" to LMM-heparin-tyramine (LMMH-Tyr-Fitc). We now describe the quantitative measurement of the plasma and blood concentrations of LMMH-Tyr-Fitc. Flow cytometry has been used to detect the plasma concentration of LMMH-Tyr-Fitc on the surface of protamine-coated latex particles and on the surface of leukocytes. The data were compared with the pharmacodynamic analysis of the antifactor Xa (aXa) and anti-thrombin activities (aIIa), measured by $\mathrm{S} 2222$ and $\mathbf{S 2 2 3 8}$ assays.

$150 \mathrm{aXa} \mathrm{IU/kg}(1,46 \mathrm{mg} / \mathrm{kg})$ of LMMH-Tyr-Fitc were injected as a bolus to 8 Sprague-Dawly rats. The blood samples were collected at 0 , $[0,30,60,120,240,360$ minutes and assayed by flow cytometry (latextest and leukocytes) and the chromogenic assays. The maximal pharmacokinetic and pharmacodynamic effects were observed at 10 minutes. The elimination rate was biphasic for plasma and blood concentrations as well as for ailla and aXa activities. The $\alpha$-balf life for blood concentration and alla activity ranged from 30 to $45 \mathrm{~min}$, for plasma concentration and aXa activity from 60 to $70 \mathrm{~min}$. The half life at the $\beta$-phase was between 66 and $83 \mathrm{~min}$. for plasma concentration and alla activity and between 125 and $143 \mathrm{~min}$ for blood concentration and aXa activity.

The results suggest that LMM-heparins have a complex pharmacokinetic and pharmacodynamic behaviour. The binding of heparin to leukocytes indicate their important role as carriers for heparin to thrombotic and inflammatory processes. The binding of heparin to protamine-coated microspheres open the possibility to detect exogenous heparin in plasma.

Supported by Deutsche Forschungsgemeinschaft (DFG), grant $\mathrm{Ha}$ $1164 / 3-1$ and $3-2$.

I. Medizinische Klinik, Fakultät für Klinische Medizin Mannheim der Universităt Heidelberg, Theodor-Kutzer-Ưfer, 68167 Mannheim

112

Anticoagulation with Low Molecular Weight Heparin (Fragmin) during Plasmaphersis. First experiences.

H. Schinzel, K. Berghoff*, I. Beuermann, R. Blank, L.S. Weilemann

II. Med. Clinic and "Institute for Clinical. Chemistry and Laboratory Medicine University Mainz, FRG

objective: Is low molecular weight heparin (LMWH) suited for anticoagulation during plasmapheresis? Which coagulation markers allow monitoring of anticoagulation? Methods: Using the single needle technique eleven plasmapheresis were performed with a capillary filter (Hemaplex BT 900). With each procedure an isovolumetric exchange of $2000 \mathrm{ml}$ patient plasma was performed. Initially, for anticoagulation a bolus of $80-90$ anti-Xa units/kg body weight of Fragmin ${ }^{R}$ was administered. Anti-factor-Xa activity (Anti-Xa), thrombin-antithrombin III complex (TAT) and prothrombin fragment $1+2$ concentration (F 1+2) were determined in the blood flowing into the capillary filter and in the filtered plasma according to a predetermined time schedule. For determination of LMWH loss through the filter a measurement of anti-Xa levels were performed in the filtered plasma.

Results: Although anti-Xa activity decreased during plamapheresis they were always found within

the therapeutic range $(>0,5 \mathrm{U} / \mathrm{L})$. The loss of Law through the filter averaged $2467 \mathrm{U}$. All procedures were without any complication. There were no signs of fibrin deposition or thrombi within the system.

F $1+2$ levels were always normal in the blood before the filter, thereafter they were pathologically increased toward the end of the plasmapheresis. The TAT concentrations increased in the blood before the filter and more pronounced in the blood behind the filter.

Conclusions: Low molecular weight heparin (Fragmin ${ }^{R}$ ) is suited for anticoagulation during plasmapheresis.

For monitoring of coagulation TAT can be regarded as the more sensitive parameter and thus seems superior to prothrombin fragment $1+2(F 1+2)$ in the early discovery of increased coagulation activation.
High Performance Size Exclusion Chromatography and Polyacrylamide Gel Electrophoresis For Characterization Of Natural And Lipophilically Unfractionated and Low Molecular Modified Glycosaminoglycans (GAGs) R. Malsch, J. Harenberg, L. Piazolo, C. Giese and D.L. Heene.

Lipophilically modified heparins are currently prepared to improve the non-antithrombin mediated effects of heparin. This paper reports on the microheterogenity of unfractionated and low molecular mass heparin (LMMH) and lipophilically derivatives using high performance size exclusion chromatography (HPSEC) and polyacrylamide gel electrophoresis (PAGE). 12 glycosaminoglycan (GAG) preparations (heparin and dermatan sulfates) were analyzed in 5 runs. The standard deviations of the mass average molecular mass $\mathrm{Mm}$ ranged from 0,65 to $20,2 \%$ and the polydispersity $P$ from 0,93 to $6,99 \%$. The standard deviations of the absorbance were between 0,01 and $0,069 \%$, the area under curve from 0,59 to $21,16 \%$ and the peak purity from 0,3 to $2,66 \%$ The molecular mass of the GAG preparations was also assayed by PAGE and performed on 5 different days. The standard deviation of the mass average molecular mass $\mathrm{Mm}$ was from 6,39 to $19,78 \%$ and polydispersity $P$ from 0,67 to $10,15 \%$.

Lipophilically derivatized heparins and low molecular mass heparins (acetyl-, benzoyl-, butyryl-, capricyl-, and octadecyl-heparin-derivatives) have been synthesized and analyzed by HPSEC and PAGE. They showed a prolonged retention time by HPSEC. Analyzing the compounds by PAGE they showed a similar migration as the parent compounds. Thus the molecular mass and the polydispersity of the lipophilically heparins and LMMH was calculated. HPSEC and PAGE allow a precise and rapid analysis of GAG preparations. The advantage of PAGE is the higher sensitivity and resolution whereas HPSEC has a better standardization and higher reproducibility. PAGE is a suitable and reproducible method to analyze lipophilically derivatized GAGs.

Supported by Deutsche Forschungsgemeinschaft (DFG), grant Ha 1164/31 and $\mathrm{Ha} 1164 / 3-2$

1. Medizinische Klinik, Fakultät für klinische Medizin Mannheim der

Universität Heidelberg, Theodor Kutzer Ufer, 68167 Mannheim.

\section{4}

COMPARATIVE STUDIES ON THE ANTICOAGULANT, ANTIPROTEASE AND ANTITHROMBOTIC ACTIONS OF RECOMBINANT TISSUE FACTOR PATHWAY INHIBITOR, ANTITHROMBIN III AND HEPARIN. J. Fareed, D. Hoppensteadt D. Callas, T.C. Wun, and J.M. Walenga. Loyola University Medical Center, Maywood, th and Monsanto Co. Chesterfield, MO.

In order to compare the relative anticoagulant, antiprotease and antithrombotic actions of recombinant tissue factor pathway inhibitor (r-TFPI). human antithrombin III (AT-III) and heparin, we used various standard experimental models. Freshly drawn normal human pooled plasma was supplemented with each agent in a concentration range of $0-25 \mu \mathrm{g} / \mathrm{ml}$. In the PT, APTT and Heptest, r-TFPI exhibited 2-20 times stronger anticoagulant actions than heparin. AT-III at these concentrations had no effect. In the thrombin time assays, r-TFPI did not show any effect, whereas heparin produced a strong anticoagulant effect and AT-III failed to produce any effect. in the thromboelastographic and whole blood clotting assays r-TFPI produced a dose dependent anticoagulant effect. $r-$ TFPI produced a much stronger anti$X a$ effect $\left(I C_{50}=0.8 \mu \mathrm{g} / \mathrm{ml}\right)$ than heparin in a plasma system. In purified systems, r-TFPI produced a strong interaction with human factor $X_{a} \| C_{50}-1.4$ $\mu g / \mathrm{ml})$, whereas neither heparin nor AT-1II produced any inhibition at concentrations up to $25 \mu \mathrm{g} / \mathrm{ml}$. r-TFPI strongly neutralized the procoagulant effects of $r$-tissue factor in whole blood and citrated plasma, whereas heparin produced a relatively weak neutralization. r-TFPl also strongly inhibited the thrombogenic effect of recombinant tissue factor in a modified jugular vein stasis thrombosis model $\left(E D_{50}=18.8 \pm 3.6 \mu \mathrm{g} / \mathrm{kg}\right.$ ), in contrast to heparin $\left(E_{50}=48.9 \pm 5.0 \mu \mathrm{g} / \mathrm{kg}\right)$ and $A T-111\left(E_{60}=>250 \mu \mathrm{g} / \mathrm{kg}\right)$. Protamine sulfate, platelet factor 4 and factor VIII did not alter the effects of r.TFPI. These results clearly indicate that $r$-TFPl is a potent antithrombotic agent which may be useful in the prophylaxis and treatment of thrombotic disorders. Furthermore, in contrast to heparin and AT-III, the biochemical and pharmacologic actions of $r$ TFPI are independent of endogenous modulation. 
115

\section{LOW INTENSITY PHENPROCOUMON - A CORRELATION OF PROTHROMBIN TIME, PROTEIN C AND SOLUBLE FIBRIN S. Haas, M. Spannagl, A. Stemberger, H.-J. Kolde, G. Blümel}

Fixed minidoses of warfarin have shown to significantly reduce the incidence of deep vein thrombosis (DVT) in patients undergoing general and gynecologic surgery. In high risk patients undergoing orthopedic or trauma surgery however, the antithrombotic efficacy of this prophylaxis has not been proven; only individually adapted higher dosages have been described which were compromised by a higher bleeding risk and a troublesome management of prophylaxis. Since phenprocoumon is the most commonly used oral anticoagulant in Germany, we aimed at developing a "low intensity phenprocoumon" regimen for prophylaxis of DVT in high risk patients. The target INR was 1,5.

Eight male and female volunteers were treated with an initial dose of 12 mg phenprocoumon ( 4 tablets) on day 1 followed by $1,5 \mathrm{mg}$ ( $1 / 2$ tablet) or $3 \mathrm{mg}$ ( 1 tablet) until an INR of 1,5 was obtained. Prothrombin time was measured daily, and the volunteers were screened for microhematuria. For the determination of prothrombin time four different thromboplastin preparations were used, and the measurements were performed in two different laboratories. There was an acceptable correlation of the prothrombin time values of all four thromboplastins used, and also the interlaboratory variation of the results was in an acceptable range. Protein C activity decreased from $97 \%$ of normal on day 1 to $53 \%$ on day 5 and increased gradually to $67 \%$ on day 11 , the last day of investigation. There was an excellent correlation of the decrease of soluble fibrin and prothrombin time from day 1 to day 9; on day 10 and 11 however, the decrease of fibrin monomers continued further although the INR remained stable with mean values of 1,5 .

On the basis of these results it may be concluded that an INR of 1,5 may be sufficient for primary prevention of DVT, however it has to be shown whether fibrin formation can also be significantly decreased in high risk patients with postoperative hypercoagulability.
116

EFFECT OF RECOMBINANT HIRUDIN AND HEPARIN ON THE FUNCTIONAL AND IMMUNOLOGIC LEVELS OF TFPI. RESULTS OF EXPERIMENTAL AND CLINICAL STUDIES. J.M. Walenga, D. Hoppensteadt, W. Jeske, J. Fareed and H.K. Breddin. Loyola University Medical Center, Maywood, IL and J.W. Goethe Universitat, Frankfurt, Germany

In order to compare the effect of $r$-hirudin and unfractionated heparin on the endogenous release of TFPI, groups of primates $(6)$ were treated with $1 \mathrm{mg} / \mathrm{kg}$ i.v. dosages. Blood samples were drawn at baseline, 15, 30, 60 and 180 mins. post drug administration. TFPI was quantitated by using a functional chromogenic method and a newly developed sandwich Elisa method. A time dependent increase in both the functional and immunologic levels of TFPI was noted in primates treated with heparin. However, despite marked anticoagulation with r-hirudin, no changes from the baseline TFPI levels in either the immunologic or functional TFPI were noted. In several clinical studies, TFPI antigen and functional levels were measured after $i . v$. heparin $(n=50)$ and low molecular weight heparin therapy $(n=50)$. While individual variations were obvious, a consistent increase in the functional and immunologic TFPI levels was observed. Patients $(n=50)$ treated with $r$-hirudin did not show a similar response. In both the primate and the clinical heparin studies, immunologic TFPI levels were always higher than the functional TFPI levels, suggesting that TFPl complexed with endogenous substances may be transformed to exhibit differential functional/immunologic ratios. These studies demonstrate that TFPI release/modulation is heparin specific and direct antithrombin agents such as r-hirudin do not alter the functional nor immunologic levels of this inhibitor.

\section{Reocclusion}

A PROLONGEO PROTHROMBOTIC STATE AFTER CORONARY STENTING IS ASSOCIATED WITH A HIGHER INCIDENCE OF LATE RESTENOSIS

U. Klaar, M. Gwechenberger, G. Chr ist, G. Zorn, D. Glogar, P. Probst and K. Huber

Intracoronary thrombus formation is a frequent ovent after coronary stent implantation and depends on the extent of mechanical trauma to the vessel wall. To prove a possible relation between an increased and/or prolonged prothrombotic state aftar stent implantation and late restenosis we investigated 20 consecutive patients after acute coronary stenting due to thrombotic reocelusion and/or dissection after coronary angioplasty (Paimaz-Schatz stent: $n=17$; Wiktor stent: $n=4$ ). We determined routine contral parameters of postinterventional anticoagulation with heparin (activated partial thromboplastin time, aPTT) and warfarin (prothrombin time. Thrombotest $\left.{ }^{(}\right), T$ ) twice daily during the first 10 days after the intervention and measur w, furthermere, in the respective samples lovels of thrombin-antithrombin complexes (TAT), prothrombin frag-ments $\left(F_{1}+2\right)$, and $D$-Dimer (DD), respectively. Presence or absence of restenosis was ovaluated by quantitative angiography at least six months after coronary stenting. Six pationts developed late restenosis ( $R$; total restenos is rate $=30 \%$ ). In patients without $R$ mean aPTT-values ranged above 60 sec at all time points of blood collection during deys 1 to 6 . Hewever, in patients with R, aPTT levols were repeatedly bolow 55 sec. Furthermoro, mean $T T$ levels (optimal therapoutic range $=5$ to $15 \%$ ) reached a value of less then $15 \%$ two days earlier (at day 5 ) in patients without $R$. Mean values of TAT, $F_{1}+2$, and DD were elevated during the first days of the folow up period in both study groups but without significant differences between them. The impressive slower respense to heparin and warfar in in patients with late $R$ can be explained by individual blecding problems in four patients of the $R$-group which forced us to raduce heparin and to increase the warfar in dosage more slowly as compared to patients without late R. Another explaration could be an individual resistance against the anticoagulative treatments used in the R-group. Our findings support the hypothesis of a pralonged prothrombotic state in the arly phase after coronary stent implantation being the trigger for pathophysiologic pathways involved in restenosis formation.

$2^{\text {nd }}$ Department of Internal Medicine, Division of Cardiology, University of Vienna, Währinger Gürtel 18-20, A-1090 Vienna, Austria
118

A PRACTICAL APPROACH TO POSIINTERVENTIONAL ANTI COAGULATION IN PATIENTS IMPLANTED WITH PALMAZSCHATZ-STENTS

A. Haushofer, W.M. Ha ibmayer, M. Ditte $]^{*}$,

H. Prachar*, J. Miczoch* and'M. Fischer

Following the implantation of coronary Pajmaz-

Schatz-s the imglantation of coronary Palmaz

with a comb inat ion of heparin, phenprocoumon and
ASS following a standard protocol. To detect changes

in clotting, specific clotting parameters (APTT, AT III in clotting, Specific clotting parameters (APIT, AT III, Heptest, PTF, TAT) were monitored at standardized time points for 10 days. The AT III activity dropped signif by bolus injection during stenting $(\mathrm{p}<0,001)$. As the hepar in dose was reduced on the fol lowing days, AT III levels increased again significantly, while APTT decreased. On day 4 PTF levels were sig. higher than on the day of stenting $(1,04 \pm 0,53 \mathrm{nmol} / 1$ vs $1,16 \pm 0,3 \mathrm{nmol}$; $0<0,005)$, but dropped during phenprocoumon medication, the difference being aga in sig. $(p<0,05)$. PTf levels showed a sig. negative correlation with At III activity showed a sig. negat ive correlation with At III activity ( $p<0,05$ ) and the Heptest $(p<0,05)$. Despite adequat ant icoagulation mean pir levels in patients showing restenosis at follow-up angiography were sig. higher $(p<0,005)$ than in those without restenosis. In one patient who developed subacute stent thrombos is clotting factors were determined 20 min prior to stent occluston. The Tevels of PTF and TAT were found to be less than the means of all other patients for this spec if ic day (PTF: $0,98 \mathrm{nmo} / / 1$ vs. $1,11 \pm 0,40$ nmol/ 1 ; TAT: $2,7 \mu \mathrm{g} / 1$ vs. $3,21 \pm 3,38 \mu \mathrm{g} / / 7$. Thus, neither PTF nor TAT predicted the occurrence of thrombot ic stent failure in the individual case. Efficient ant icoaqulation by a combination of anticoagulants is imperative for stent implantation.

The ant icoaguiant regimen and the monitoring concept used were found to be both effective and safe.

Zentrallabor - KH Lainz, * 4.Med. Abt mit Kardiologie u. Ludwig Boltzmann-Inst itut für Herz infarktforschung $\mathrm{KH}$ Lainz, Wolkersbergenstr. 1 , A-1130 Vienna, Austria 
HOW DOES HEPARIN ENHANCE THROMBOLYSIS ?

R. Dechend ${ }^{2}$, S. Hauck ${ }^{2}$, K. - J. Osterziel ${ }^{2}$, W. Gödecke ${ }^{2}, M$. Praus ${ }^{2}$, M Barthels ${ }^{1}$. R. Dietz ${ }^{2}$. D.C. Gulba ${ }^{2}$. Medizinische Hochschule Hannover (1) \& UKRV Franz-Volhard-Klinik. Berlin (2)

Low molecular weight (Imw) and high molecular weight (hmw) heparins. both have been proven to stimulate the fibrinolvtic potency of fibrin-specific plasminogen-activators (FS-PA). This has been demonstrated both in anima models and clinical studies. To investigate the underlying mechanism of action. we studied 12 healthy volunteers in an open-labeled three armed cross-over study. They were treated in a random fashion with $7500 \mathrm{IE} \mathrm{hmw}$ (Liquemin), $2500 \mathrm{E}$ lmw heparin (Mono-Embolex) or placebo. the wash out period between treatments being 6 weeks. Samples were collected at 8 defined times within 2 hours after injection and factors of the extrinsic and intrinsic fibrinolytic systems were determined. For statistics mulivariance analysis (MANOVER) was applied.

\begin{tabular}{|l|c|c|c|c|c|c|}
\hline parameter & \multicolumn{2}{|c|}{ placebo } & \multicolumn{2}{c|}{ LMW-heparin } & \multicolumn{2}{c|}{ HMW-heparin } \\
\hline time & 0 & $2 \mathrm{~h}$ & 0 & $2 \mathrm{~h}$ & 0 & $2 \mathrm{~h}$ \\
\hline PLG $(\%)$ & 103 & 101 & 92 & 93 & 92 & 102 \\
\hline$\alpha_{2}$-APL $(\%)$ & 105 & 102 & 97 & 99 & 99 & 102 \\
\hline $\mathrm{t}-\mathrm{Pa}(\mathrm{ng} / \mathrm{ml})$ & 3.3 & 3.7 & 5.0 & +8 & 4.7 & 4.7 \\
\hline PAI $(\mathrm{AU} / \mathrm{ml})$ & 13.9 & 14.5 & 15.7 & 13.5 & 17.1 & 15.5 \\
\hline Prot C $\%)$ & 83 & 87 & 86 & 84 & 88 & 87 \\
\hline Prot S $\%$ ) & 90 & 89 & 101 & 106 & 97 & 98 \\
\hline PK $(\%)$ & 94 & 96 & 93 & 94 & 90 & 91 \\
\hline F XII $(\%)$ & 100 & 102 & 103 & 105 & 102 & 102 \\
\hline$\alpha$-FXII Inh $(\%)$ & 106 & 107 & 107 & 110 & 107 & 111 \\
\hline$\beta$-FXII Inh $(\%)$ & 105 & 108 & 108 & 110 & 106 & 109 \\
\hline TAT $(\mu$ g/mi) & 16.3 & 37.7 & 12.8 & 10.1 & 8.1 & 8.9 \\
\hline
\end{tabular}

$\mathrm{Pk}=$ Präkallikrein, TAT $=$ Thrombin-Antithrombin complex

Conclusions: The endogenous intrinsic and extrinsic fibrinolytic systems is not directly stimulated neither by $1 \mathrm{mw}$, nor by hmw heparin. The stimulating effect of heparin on thromboiytic agents therefore must most probably be mediated through inhibition of the coagulant activity present.

present address: Klinikum Rudof Virchow. FU Berlin. Franz-Volhard-Klinik \& Max Delbrück Centrum für molekulare Medizin. Wiltbergstr. 50

13125 Berlin
ASPIRIN DOSE AND PROGRESSION OF PERIPHERAL VASCULAR DISEASE: RESULTS OF A CONTROLLED CLINICAL TRIAL

C. Ranke, H. Hecker, A. Creutzig, K. Alexander

High doses of aspirin can retard the progression of peripheral arterial occlusive disease (PAOD), but the efiect of low doses of aspirin on the course of PAOD is unknown. We performed a prospective, randomized, controlled clinical trial to compare $50 \mathrm{mg}$ of aspirin with a dose of $900 \mathrm{mg}$ per day with respect to progression of atherosclerosis and with respect to patency rates after percutaneous transluminal angioplasty (PTA) in 359 patients with PAOD.

After one year follow-up, patency rate as well as an arteriographic score were not different in both treatment groups in the overall analysis. As a global measure of disease progression we performed linear regression analyses using the time course of each individual patients Doppler-derived systolic ankle pressure values. The $b$ values (slope) were then calculated for all patients. The effect of aspirin dose, gender, and risk factors on disease progression was evaluated for the semiquantitative arteriographic score as well as for the Doppler-derived $b$ values. Regression slopes were not different in male patients with respect to aspirin dose, but women showed a favorable outcome under $900 \mathrm{mg}$ aspirin treatment $\left(b=8 \cdot 10^{-3}\right)$ as compared to a falling pressure slope under the low dose regimen $(b=-$ $\left.8 \cdot 10^{3} ; p=0,02\right)$. Current smoking was associated with disease progression estimated arteriographically in female patients under low dose aspirin treatment. This effect was observed only in the proximal and distal vessel segments, and not at the angioplasty site. The explanatory analysis of our data shows dose effects of aspirin in female patients, especially in the case of current smoking.

This favorable effect of high dose aspirin cannot be explained by its antiplatelet activity due to platelet cyclooxygenase inhibition, but results of stroke prevention trials and carotid ultrasound studies suggested dose dependent aspirin effects, too.

Dr. C. Ranke, Division of Angiology, Hannover Medical School, Konstanty-Gutschow-Str. 8, 30625 Hannover, Germany.

CONTROLLED TRIAL OF HIGH VERSUS LOW DOSE ASPIRIN TREATMENT AFTER PTA IN PATIENTS WITH PERIPHERAL VASCULAR DISEASE

A.Creutzig, C.Ranke, G.Luska, H.H. Wagner, M.Galanski, s. Bode-Böger, J.Frölich, H.Hecker, H.J.Avenarius, R.Alexander

Percutaneous transluminal angioplasty of aortoiliac and femoropopliteal atherosclerotic lesions can provide long-lasting hemodynamic improvement. High-dose aspirin treatment was shown to be effective as reocclusion prophylaxis, but low doses of aspirin are preferable because of fewer adverse effects. We performed a double-blind, randomized, controlled clinical trial in patients with peripheral vascular disease with lesions appropriate for angioplasty. We compared the efficacy and side-effects of two doses of aspirin $(50 \mathrm{mg}$ vs $900 \mathrm{mg}$ daily) during a period of 12 months after angioplasty. A total of 359 patients were evaluated, and 175 patients were randomly assigned to treatment with $900 \mathrm{mg}$ aspirin daily, 184 received $50 \mathrm{mg}$ aspirin a day. Thirty-nine patients developed restenosis at the angioplasty site, the cumulative percentage of event-free survival after one year (patency rate) was $84.9 \%$ in the 900-mg group and $83.8 \%$ in the $50-\mathrm{mg}$ group. Both treatment groups were equivalent with respect to their restenosis rates. Nine patients $(5 \%)$ of the 900-mg group had serious gastrointestinal side effects (peptic ulcer, 8 patients; erosive gastritis requiring transfusion, 1 patient) as compared to 2 patients (1\%) with peptic ulcer in the 50-mg group: $p=0.03$. The results of our study show that a dose of $50 \mathrm{mg}$ a day is as effective as $900 \mathrm{mg}$ aspirin in the prevention of restenoses after lower limb angioplasty, and severe gastrointestinal side effects are less frequent.
DOUBLE BLIND PLACEBO-CONTROLLED PROSPECTIVE RANDOMIZED STUDY OF ACETYLSALICYLIC ACID (ASS) OR MOLSTDOMTNE IN PTCA: EFFECTS ON HEMOSTASIS

H.Riess, V. Ding-Reinelt, G.Himmelreich, C.Hehlert-Friedrich, and E.Fleck

The antithrombotic effect of ASS given to reduce the acute reocclusion rate after PTCA seems incomplete because of the missing inhibition of platelet adhesion and the inhibition of prostaglandin (PG) $\mathrm{I}_{2}$ in the vessel wall Furthermore there is evidence that ASS may decrease the release of tissuetype plasminogen activator (t-PA). Molsidimine inhibits platelet function by raising CGMP without impairment of endothelial $\mathrm{PGI}_{2}$, synthesis. In addition molsidomine may increase fibrinolytic activity.

Patients with coronary artery disease making elective PTCA necessary were included in a placebo-controlled, prospective and randomized study. We compared a control group $(\mathrm{n}=24)$ with two groups treated with $3 \times 100 \mathrm{mg}$ ASS $(n=23)$ or $3 \times 8 \mathrm{mg}$ molsidomine $(n=23)$ before PTCA. Immediately before PTCA patients received 500mg ASS, $2 \mathrm{mg}$ SIN-1 (active metabolite of molsidomine) or $5 \mathrm{ml}$ placebo i.v, respectively. release (ATP-R) measured in whole blood and platelet rich plasma and the fibrinolytic system (t-PA and plasminogen activator inhibitor (PAI)) were investigated.

Results: 1. The PTCA procedure resulted in a significant shortening of the lag-phase as well as in an inconstant increase of maximal amplitude in PA. BT after PTCA was significantly prolonged.

2. ASS reduced PA and ATP-R but the lag-phase shortening was not prevented. BT became significantly prolonged as compared to placebo.

3. Molsidomine prevented the procedure-dependent shortening of the lag phase and showed an inconstant (and minor, as compared to ASS) inhibitory effect on PA and ATP-R. BT did not differ from the placebo group.

4. ASS and molsidomine did not influence the fibrinolytic system.

A study of the combined antiplatelet approach with molsidomine and ASS seems justified.

Medizinische Klinik und Poliklinik, Universitätsklinikum Rudolf Virchow and Deutsches Herzzentrum Berlin, Augustenburger Platz 1, 13353 Berlin
Bleeding time (BT), collagen-induced platelet aggregability (PA) and ATP- 
Fibrinolysis

123

\section{PLASMINOGEN ACTIVATORS (PAs) IN HUMAN ATHERO- SCLEROTIC VESSEL WALL}

\section{T. Padro, M. Steins, W. Krings, and J. Kienast}

To investigate the potential involvement of PAs in atherogenesis, we have comparatively studied antigen and functional levels of PAs of the urokinase- (U-PA) and tissue-type (t-PA) in protein extracts of human aorta (obtained from autopsies) and coronary arteries (heart explants) with and without atherosclerotic lesions. In addition, tissue specimens from femoropopliteal atherectomies (FPA) were analyzed. In coronary arteries, both u-PA and t-PA antigen were significantly increased in segments with fibrous plaques (FP) as compared to areas without lesions. This increase was associated with a doubling of the $u-P A$ : $t-P A$ antigen ratio $(p$ $<0.001)$ ). Similar results obtained in aortic vessel wall demonstated that the U-PA ag increase in lesional areas was restricted to the intima, whereas t-PA ag increased mainly in the inner media layer and core region of FP. Free PA activity was detected in intima/media extracts of coro-nary arteries and was largely attributable to the presence of active t-PA $(r=0.83 ; p<$ 0.001 ). LP(a) was significantly increased in FP as compared to areas without lesions $(p<0.01)$. In coronary arteries and FPA samples, $L p(a)$ levels were positively correlated to $u-P A(r=0.4-0.6$ $p<0.05)$ and plasminogen content $(r=0.4-0.5 ; p<0.05)$. The data suggest different roles for PAs of the tissue- and urokinase-type in atherosclerotic remodelling of the arterial vessel wall.

Department of Internal Medicine, University of Münster, AlbertSchweitzer-Str. 33, 48129 Münster

125

INCREASED PLASMA ACTIVITY OF PLASMINOGEN ACTIVATOR INHIBITOR-1 IN PATIENTS WITH PRIMARY PULMONARY HYPERTENSION.

M. Sosada, M. Höper ${ }^{\star}$ H.J, Avenarius, M. Barthels, F. Bergmann, H.'Fabel ${ }^{\star}$, J. Pieper, T. Wagner ${ }^{*}$ and $H$. Poliwoda.

Thrombosis in small pulmonary vessels or thrombi coexistent with arteriopathy are frequent findings in patients with primary pulmonary hypertension (PPH). Whether this is primarily involved in the pathogenesis of this disease or occurs as a result of increased shear stress and diffuse damage of pulmonary endothelium is unknown yet. Nevertheless. several data support a beneficial effect of anticoagulation. In previous determination of fibrin degradation products, fibrinogen degradation products, prothrombin fragment $1+2$ and thrombin-antithrombin III complex we were not able to find significant increase of these parameters in a group of 8 patients with PPH. Data of meanwhile 16 patients with this rare disease confirmed that there is no increase in the above mentionned values. But in 15 of these 16 patients with advanced PPH (NYHA III-IV, 6 male, 10 female, mean pulmonary artery pressure $60,1 \pm 16,0 \mathrm{mmHg}$, mean cardiac index $1,6 \pm 0,35$ $1 / \mathrm{min} / \mathrm{m}^{2}$ ) we found increased PAI-1 levels of $27,43 \pm 7,60 \mathrm{AU} / \mathrm{ml}$ (normal $<17 \mathrm{AUJ} / \mathrm{ml}$ ). No direct correlation was detected between hemodynamic parameters and the level of PAI-1. In conclusion elevated PAI-1 levels were proven in our patients with severe PPH. This indicates an impaired fibrinolytic activity in this disease.

Div. of Hematology and Oncology, Div. of Pneumology , Hannover Medical. School, D-30623 Hannover

RELATIONSHIP OF PLASMINOGEN ACTIVATOR INHIBITOR ACTIVITY, TISSUE-TYPE PLASMINOGEN ACTIVATOR AND VON WILLEBRAND FACTOR CONCENTRATION TO AGE, SEX AND OTHER RISK FACTORS FOR CORONARY HEART DISEASE

\section{G. Siegert, S. Bergmann, W. Jaross}

The dependence of plasminogen activator inhibitor activity (PAI), tissue-type plasminogen activator antigen (t-PA) as well as von Willebrand factor antigen (VWF) on age, gender, body mass index (BMI) and lipoprotein metabolism parameters was studied in a cohort of the population of Dresden (Drecan study). PAl activity was determined in 2662 males and females, t-PA as well as VWF concentration were measured in an age related sub population of 900 probands. PAl activity and t-PA concentration were found to increase until middle age in men and ofd age in women. VWF concentration increased only in the old-age-group. PAl activity and t-PA concentration were higher in men than in women, VWF concentration showed no sex difference. PAl activity was strongly correlated with BMI and triglyceride concentration (TG) as well as t-PA concentration. t-PA concentration showed a strongly correlation with BMI, LDL-cholesterol and PAI activity. WWF concentration was correlated with BMI and t-PA concentration. In women taking oral contraceptives the PAl activity and the tPA concentration were lower than in women who did not. The influence of oral contraceptives on PAI activity was independent of influence of BMI and TG concentration and was not the only responsible factor of sex difference of PAI activity. The influence of oral contraceptives on t-PA concentration was dependent on the influence of PAI activity on t-PA concentration. These results point to a strong relationship between PAI activity, $t-P A$ and WWF concentration and other risk factors for coronary heard disease.

Present address: Dr. med. habil. G. Siegert

Technical University Dresden

Faculty of Medicine Can Gustav Carus

Institute of Clinical Chemistry and Laboratory Medicine

Fetscherstraße 74

01307 Dresden
Findings concerning lipid metabolism, hemostasis and fibrinolysis in patients with and without coronary artery stenosis

W. Withold ${ }^{1}$, M. Heins $s^{2}$, F.-C. Schoebel ${ }^{2}$, D. Stein ${ }^{2}$, K. Pels ${ }^{2}$, R. Behnke ${ }^{2}$, M. Leschke ${ }^{2}$, B.E. Strauter ${ }^{2}$, H. Reinauer'

Institute for Clinical Chemistry and Laboratory Diagnostics

${ }^{2}$ Clinic for Cardiology

University of Duesseldorf

At present various parameters of lipid metabolism (f.i. LDL cholesterol, lipoprotein [a]) are considered as cardiovascular risk factors; moreover, in patients at high risk alterations of hemostasis and fibrinolysis may be found and interactions between hemostasis and lipid metabolism are discussed.

We examined the diagnostic validity of different parameters of lipid metabolism, hemostasis and fibrinolysis in the diagnosis of coronary artery stenosis in 229 consecutive patients with and without coronary artery stenosis (as documented angiographically). The prevalence of coronary artery stenosis amounted to $62 \%$. The following analytes of lipid metabolism were determined: total cholesterol, triglycerides, HDL cholesterol, LDL cholesterol and lipoprotein (a) (lpa). Besides the following quotients were calculated: LDL/HDL cholesterol and total cholesterol/HDL cholesterol. Moreover the following parameters of hemostasis and fibrinolysis were determined: fibrinogen, prothrombin fragment $F_{1+2}$, tissue plasminogen activator (tpa) (employing ELISA technique) and plasminogen acticator inhibitor (as determined functionally).

Patients with coronary artery stenosis were characterized by higher concentrations of lipoprotein (a) and of tissue plasminogen activator compared with patients without coronary artery stenosis $(p<0.05)$ whereas the other parameters did not show any significant differences in both patient groups $(p>0.05)$

There was no correlation between Ipa and tissue plasminogen activator $(r=-0.05, p>0.1)$. Assuming a decision level of $48 \mathrm{mg} / \mathrm{dl}$ lipoprotein (a) and of $10 \mu \mathrm{g} / \mathrm{l}$ tissue plasminogen activator the diagnostic validity of these parameters was quantitatively characterized as follows: diagnostic sensitivity $=25.2 \%$ (lpa) and $21.5 \%$ (tpa), diagnostic specificity $=$ $89.5 \%$ (lpa) and $86.2 \%$ (tpa), positive pv $=79.1 \%$ (lpa) and $71.9 \%$ (tpa) as well as negative $\mathrm{pv}=43.3 \%(\mathrm{lpa})$ and $40.0 \%$ (tpa). 
Fibrinolysis in coronary artery disease

M. Heins, F.-C. Schoebel*, W. Withold, D. Stein*, K. Pels*, T. Jax*, M. Leschke*, B. E. Strauer*, H. Reinauer

Institut für Klinische Chemie und Medizinische Klinik und Poliklinik B*, Heinrich Heine Universität Düsseldorf

Disturbances of the fibrinolytic system are considered to be a predictor for the incidence of cardiovascular events. The relationship between fibrinolytic parameters and the extent and severity of coronary atherosclerosis has not been investigated systematically at the present time.

In 105 consecutive patients (pat.) with angiographically documented coronary arfery disease $(C A D)$ plasminogen activator inhibitor $(\mathrm{PAI})$, whole tissue plasminogen activator (t-PA) were measured. The severity (coronarscore) and the extent (number of significantly stenosed vessels) of $\mathrm{CAD}$ were determined by means of coronary angiography. t-PA was significantly increased in pat. with coronary 3-vessel disease $(9.4 \pm 3,1 \mathrm{ng} / \mathrm{ml})$ compared with pat. with non-significant coronary sclerosis (extent of stenosis $<50 \%)(5.0 \pm 1.7 \mathrm{ng} / \mathrm{ml})(p<0,05)$ and nearly significantly in 1 -vessel $\mathrm{CAD}(8.2 \pm 3.0 \mathrm{ng} / \mathrm{ml})(\mathrm{p}=0.09)$. There was a positive correlation between t-PA and the overall score $(r=0.25, p<0.05)$ as well as the score for the proximal segments $(1=0.27, \mathrm{p}<0.0 \mathrm{I})$. The t-PA concentration correlated highly significantly with the PAI activity $(r=0.37, p<0.0001)$. In lipid parameters PAI $(r=0.51, p<0.001)$ and $t-P A(r=0.28, p<0.01)$ correlated significantly positive $(p<0.01)$ with the triglyceride values respectively.

Conclusion: In patients with CAD there is a significant interrelationship between CAD and the severity as well as the extent of coronary sclerosis. This results let us assume a secondary activation of the fibrinolytic system in CAD. In patients with hypertriglycerideemia these changes are characterised by an additional increased potential of inhibitors of fibrinolysis.
P53 TUMOR SUPPRESSOR REGULATES DIFFERENTIALLY THE U-PA, t-PA, and PAI-1 GENE TRANSCRIPTION

D. von der Ahe and C. Kunz

Two types of serine proteases, urokinase-type (-u-PA) and tissue-type (tPA) plasminogen activator are known to convert plasminogen into plasmin. Considerable experimental evidence has indicated that the production of plasminogen activators by tumor cells and the generation of plasmin is associated with various aspects of malignant transformation, tumor invasion and metastasis. In normal cells the plasminogen activators and their inhibitors (PAl-1, PAl-2) are strictly regulated, mainly at the transcriptional level. During our studies of U-PA gene regulation by several onco-proteins, we found out that the U-PA enhancer $(-2000$ to -1870$)$ and promoter are negatively regulated by the tumor suppressor gene product p53. The gene for the nuclear phosphoprotein is the most commonly mutated gene yet identified in human cancers. There is accumulating evidence that p53 may regulate gene transcription. To identify directly the elements necessary for repression by $\mathrm{p} 53$, celis (Hela, Saos-2, HT1080, CV-1) were transiently cotransfected with $\mathrm{p} 53$ wild-type and mutant expression vectors (transregulators) and plasmids containing the U-PA, t-PA, and PAl-1 promoter and enhancer sequences cloned upstream of the chloramphenicol acetyltransferase (CAT) gene. The U-PA-CAT and t-PA-CAT reporter genes are transcriptionally suppressed by $\mathrm{p} 53$ co-expression in all cell types, whereas the PAl-1 promoter is not repressed or slightly activated. When the PAl-1 promoter is progressively deleted we found that the -216PAl-1-CAT is not repressed and the -142PAI-1-CAT is repressed by p53 to very low basal levels. Within the $\mathrm{p} 53$ sensitive PAl-1 promoter positions -216 and -142 upstream the transcription start site we detected a putative p53 binding site. Currently we are characterizing this putative $p 53$ binding site biochemically by protein-DNA binding studies. The study of the regulation of extracellufar protease and inhibitor genes by a tumor-suppressor gene may have some relevance in the context of the link between cellular transformation and tumor progression.

Hemostasis Research Unit, Kerckhoff-Klinik, MPI, Sprudelhof 11, D-61231 Bad Nauheim, Germany

Platelets

OPTIMIZATION OF EXPERIMENTAL CONDITIONS FOR THE STUDY OF PLATELET ACTIVATION BY FLOW CYTOMETRY. M.J. Koza, J. Jato, T.V Shankey, J.M. Walenga, R. Pifarre, J. Fareed. Dept. of Thoracic and Cardiovascular Surgery, Pathology and Urology, Loyola University Medical Center, Maywood IL 60153.

Several papers have been published regarding the use of flow cytometry to measure platelet activation in vitro and in certain clinical situations. Our laboratory has extensively studied various methods of collection and sample preparations. Normal human donors $(n=4)$ were carefully drawn into EDTA, citrate and r-hirudin for whole blood), supplemented with saline, arachidonic acid, adenosine diphosphate, collagen, epinephrine or ristocetin and incubated at either $25^{\circ} \mathrm{C}$ or $37^{\circ} \mathrm{C}$. WB aliquots were taken, fixed in $1 \%$ paraformaldehyde, washed and labeled with direct monoclonal antibodies to glycoprotein Illa (CD61-FITC) or glycoprotein llbilla (CD41-FITC) and granular membrane protein 140 (CD62-PE). Samples were then measured for CD62 expression. R-hirudin demonstrated the least amount of activation in the saline control compared to citrate and EDTA. Loss of the CD41 and CD61 signal was seen with EDTA samples at $37^{\circ} \mathrm{C}$. Differences were also seen between the two temperatures. Certain aspects of platelet activities were not seen in samples prepared for PRP or those at $25^{\circ} \mathrm{C}$. This data demonstrates that blood carefully collected in $r-$ hirudin for ex vivo WB anaiysis is the optimal method of collection and in vitro experiments performed at $37^{\circ} \mathrm{C}$ would provide more physiologic relevant results.
BLEEDING TIME AND PLATELET FUNCTION IN FAWN HOODED RATS AND WISTAR RATS AFTER INFUSION OF DDAVP (MINIRIN ${ }^{R}$ )

A. Pößnecker, C.M. Kirchmaier, H.K. Breddin

Bleeding Fawn-Hooded rats (FH rats) with a platelet storage deficiency similar to that described in man were evaluated as a newly developed animai model of primary hemostasis. Wistar rats were used to compare with the findings in the $\mathrm{FH}$ rats.

Male FH rats of $310-420 \mathrm{~g}$ and male Wistar rats of $300-350 \mathrm{~g}$ were intraperitoneally anaesthetized with $60 \mathrm{mg} / \mathrm{kg}$ pentobarbital sodium. Intravenous catheters were implanted into the v.jugularis and the v.femoralis for infusion and blood sampling to investigate platelet functions (platelet count, shape change, changes in volume distribution, F VIII and VWF). After taking reference samples and a 10 -minute infusion of DDAVP $(0.2,0.4$, and $0.8 \mu \mathrm{g} / \mathrm{kg}$ body weight), the bleeding time was investigated 15 and 30 minutes after the end of the infusion and then after 1,2 , and 3 hours, using the skin bleeding time (Simplate 1) by cutting into the horizontally lying rat tail. The blood sampling for investigating the platelet function parameters was carried out at the same time.

Before infusion the bleeding time in FH rats ranged from $4^{\prime 2} 26^{\prime \prime}$ to $5^{\prime} 48^{\prime \prime}$. After infusion the bleeding time was markedly shortened and the effect lasted for more than three hours. The shortest bleeding times were observed 30 and 60 minutes post infusion. Platelet counts and platelet volume distribution were not affected. The platelets were activated dose-dependently after infusion and the effect lasted longer than three hours. The F VIII- and vWF-values increased markedly.

In Wistar rats the mean bleeding time decreased down to $1{ }^{\prime 2} 20^{\prime \prime}$ after one hour post infusion with mean pre-infusion values of $1^{\prime \prime} 46^{n}$ to 2 minutes. Platelet counts were not affected. The platelets were activated similar to those of the $\mathrm{FH}$ rats.

Platelets react similar in $\mathrm{FH}$ rats and Wistar rats, comparing the results of the $\mathrm{FH}$ rats with the obtained results of the Wistar rats. In both strains the bleeding time decreased with the shortest time at one hour and the platelets were activated up to 45 to $50 \%$ while the number of platelets was not affected.

In FH rats the bleeding time may be shortened by different mechanisms. After intravenous DDAVP injection this rat strain shows increased FVIII and WWF values, similar to the increases of F VIII and VWF seen in man with platelet disorders.

Thus the shortened bleeding time in FH rats is propably due to an activation of platelets and an enhanced production of VWF.

Center of Intemal Med., Dept. of Angiology, J.W. Goethe-University, Th.Stem Kai 7, 60590 Frankfurt/M. 
Effects of Intravenous Magnesium on Platelet Aggregation and Binding of Fibrinogen and GMP140 to the Platelet Membrane M.Gawaz, I.Ott, S.Mehringer, F.-J.Neumann

Magnesium $(\mathrm{Mg})$ deficiency and its association with platelet hyperactivity has been well recognized in a variety of diseases including myocardial infarction, preeclampsia, or diabetes. The effect of $\mathrm{Mg}$ on platelet function in vivo was studied in 10 healthy volunteers before and just after infusion of $8 \mathrm{mmol} \mathrm{MgSO} 4$. Platelet aggregation was determined in platelet-rich plasma using ADP as agonist to activate platelets in final concentrations (of 0.3 , 0.6 , and $1.2 \mathrm{mM}$ ). In parallel, ADP-induced exposure of fibrinogen and GMP-140 on the platelet membrane was determined by use of monoclonal antibodies, anti-Fg and anti-CD62, and flow cytometric techniques. Intravenous $\mathrm{Mg}$ inhibited significantly ADP-induced platelet aggregation $(\mathrm{p}<0.05)$ by approximately $40 \%$. The decrease in platelet aggregation was associated with a significant decrease in Fg binding and GMP140 expression.

\begin{tabular}{|lll|}
\hline & Pre & Post \\
Aggregation $^{1}$ & 100 & $62.8 \pm 6.9$ \\
Fg binding $^{2}$ & $278.4 \pm 16.7$ & $246.0 \pm 16.5$ \\
GMP140 expression $^{2}$ & $282.3 \pm 18.5$ & $251 \pm 19.7$ \\
\hline
\end{tabular}

$*, \#,+\mathrm{p}<0.05 ; 1 \%$ of maximal aggregation; 2 mean intensity of fluorescence

The present data shows that intravenous $\mathrm{Mg}$ inhibits ex vivo platelet aggregation effectively. Thus, the here described antiplatelet effects of extracellular $\mathrm{Mg}$ might be pathophysiologically important in treatment of diseases with enhanced platelet activity.

I. Medizinische Klinik der Technischen Universität München

132

123-I-MIBG: AN AGENT FOR PHYSIOLOGICAL

PLATELET LABELLING

E.Conrad, G. Endert and P.Kleinert

The goal of this investigation was to determine the platelet uptake (LE) of 123-I-MIBG of two suppliers. We incubated the human platelets in saline and plasma getween 10 and 90 min. The platelet number was about $4 \times 10^{\circ}$, the incubation volume was varied between 0.5 and $5 \mathrm{ml}$. The stability of labelling was determined between $2 \mathrm{~min}$ and $24 \mathrm{hrs}$. The results showed a saturation of uptake after 40 to 50 min. There was no difference in plasma or saline: LE of $70 \%$ in $0.5 \mathrm{ml}$ after $40 \mathrm{~min}$. The stability of the label was determined in plasma, incubation time between $15 \mathrm{~min}$ and $24 \mathrm{hrs}$. There was a drop of the platelet-bound activity down to $25 \%$ after an incubation of $21 \mathrm{hrs}$.

"Cold" MIBG reduced the platelet uptake from $70 \%$ to $30 \%$, a higher activity with a higher amount of MIBG reduced the platelet uptake, too.

The optimal method to label platelets is to use an incubation volume of $0.5 \mathrm{mI}$ and to incubate 40 to $50 \mathrm{~min}$ in plasma. This method shows no advantage in comparison to oxine or tropolone with regard to $L E$, however, the radiation characteristics favours 123 -iodine.

In order to study platelet kinetics in vivo 123-I-MIBG does not seem to be an appropriate agent because of the significant washout.

Department of Nuclear Medicine, Medical High School Erfurt Nordhäuser Str.74, 99084 Erfurt
133

EFFECTS OF 14 DAY THERAPY WITH DIFFERENT DOSES OF
ASA ON BLOOD COAGULATION AND PLATELET FUNCTIONS.

P.Radziwon*, J.Schenk,B.Boczkowska-Radziwon, H.K.Breddin

Aspirin (ASA) is widely used to prevent in the atherosclerotic patients. $10-15$ years ago $500 \mathrm{mg}$ ASA daily was the recommended dose. Today $100 \mathrm{mg}$ daily is the standard prophylactic amount of ASA. In spite of this low dose of ASA there are patients who do not tolerate it.

The aim of our study was to compare the effect of different doses of ASA (50 mg, $100 \mathrm{mg}, 300 \mathrm{mg}$, and $500 \mathrm{mg}$ ) on platelet functions and blood coagulation in patients during a 14 day treatment period. The following parameters were studied: collagen induced aggregation, adhesion to siliconized glass, platelet induced thrombin generation time (PITT), aPTT, PT, fibrinogen on days $1,3,8$ and 14 before and 3 hours after the intake of a single dose of ASA. Collagen induced aggregation was the most sensitive parameter to ASA, but its changes were similar in all groups and no cumulative effect was observed after 14 days of ASA-treatment Miean PITT values were shorter 3 hours after intake of ASA than before. The values on day 14 were similar to those at the beginning of the study. Platelet adhesion was slightly but not significantly decreased during the treatment period. There was no dose dependency of this effect. aPTT, PT. AT III and fibrinogen showed no changes during the ASA therapy in group of patients. The effect of $50 \mathrm{mg}$ and $100 \mathrm{mg}$ (standard dose) ASA on blood coagulation and platelet functions during 14 day therapy was similar. It could be very interesting also to compare the prophylactic efficacy of these two doses in a prospective study.

Dept. of Internal Medicine, Division of Angiology, J.W.GoetheUniversity, T.Stern-Kai 7, 60590 Frankfurt am Main, FRG

* Dept. of Haematology, Medical School, Bialystok, Poland
134

PLATELET ACTIVATION AND THROMBIN GENERATION IN "PLATELET-INDUCED THROMBIN GENERATION TIME" (PITT) P.Radziwon*, B.Boczkowska-Radziwon, J.Schenk, M.BasicMicic, H.K.Breddin.

In our newly developed global coagulation assay - Plateletinduced thrombin generation time (PITT) a small amount of PRP obtained from partialy anticoagulated blood is rotated in a disc shaped cuvette within the light beam of a photometer. The changes in optical density are recorded. During the rotation platelets are activated and release reaction occurs. Thrombin is formed at the platelet surface and at the cuvette walls and triggers coagulation. To clarify the reactions occuring in the rotating cuvette we measured at different time intervals from the start of rotation thrombin generation in terms of thrombin proteolitic activity against the chromogenic substrate S-2238 and the prothrombin fragment $\mathrm{F}^{1}+2$ generation. Platelet adhesion to the cuvette wall was observed microscopically and qualitatively estimated. Platelet release of B-TG and PF-4 was measured using B-TG and PF-4 ELISA-kits (Boehringer Mannheim).

The first change observed in the cuvette is platelet adhesion starting during the first minute of rotation followed at about the 3d minute by the release of B-TG and PF-4 from platelets. From the 8th minute on we detected increased concentrations of $F 1+2$. The proteolysis of $\mathbf{S}-2238$ was only observed shortly after the plasma sample had been clatted in the cuvette. Our results demonstrate that in PITT activated platelets are necesscary for thrombin generation and coagulation. Thrombin generation corresponds to the degree of platelet activation.

Dept. of Internal Medicine, Division of Angiology, J.W.GoetheUniversity, T.Stern Kai 7, 60590 Frankfurt am Main, FRG *Dept. of Haematology, Medical School, Bialystok, Poland 
THE INFLUENCE OF HEPARIN ON PLATELET ADP AND PLATELET FACTOR 4 RELEASE AND THE EXPRESSION OF GPIIB/IIIA

N. Höllger, J. Storck and R.E. Zimmermann

In the present study we present results concerning the pathogenic mechanism of heparin induced thrombocytopenia type 1. An ELISA was developed to measure directly the expression of Glycoprotein GPIIb/llia (GP/lb/lla) in the presence and absence of ADP and under the influence of various heparins. In addition, the release of ADP (luciferin-luciferase assay) and platelet factor 4 (PF4-ELISA) was measured in platelet rich plasma.

Heparin also induced the expression of GPllb/llla with and without prior stimulation with ADP. On the other hand we could demonstrate that the addition of heparins and protaminsulfate to platelets resulted in a significant release of intracellular stored ADP from $60 \mathrm{nmol} / \mathrm{ml}$ to $90-120 \mathrm{nmol} / \mathrm{ml}$ and PF4 from $29 \mathrm{ng} / \mathrm{ml}$ to $38-56 \mathrm{ng} / \mathrm{ml}$. These results suggested, that heparin(oid)s modulate the expression of GPIIb/llla with ADP as a mediator.

Universität Münster, Institut für vegetative Physiologie, Robert Koch Straße 28,48149 Münster
SHEDDING OF PLATELET MICROVESICLES REQUIRES PLATELET AGGREGATION

\section{A. Ruf, H. Patscheke and E. Morgenstern*}

Platelets shed microvesicles (MV) with procoagulant activity upon stimulation with various agonists. Recent flow cytometric data have shown that shedding of MV can be abrogated by antibodies against the Gpllb/lla complex, RGDS-peptides, and EDTA which prevent platelet aggregation by blocking fibrinogen binding. (Gemmell C.H., et al: (1993) Thromb. Haemostas. 69:1017). However, the question has not been elucidated whether fibrinogen binding or the aggregation process is crucial for the formation of MV. We used a methodological approach that allowed us to determine absolute MV and platelet counts in a flow cytometer with a volume controlled flow system (Cytoron Absolute). Hence, in contrast to other flow cytometers measuring relative MV and platelet counts, MV could be quantified independently of the platelet concentration and its decrease upon platelet aggregation. Platelet-rich plasma was adjusted with plateletpoor plasma to a platelet concentration of $10^{7} / \mathrm{ml}$ and stimulated with U46619 $2 \mu \mathrm{mol} / \mathrm{h}$ or $\mathrm{A} 231871 \mu \mathrm{mol} / \mathrm{h}$ for $10 \mathrm{~min}$. MV were identified immunologically with FITC-labeled anti-GPIb antibodies and discriminated from platelets and aggregates by their forward light scatter signal and their green fluorescence intensity. In some experiments, platelet expression of bound fibrinogen was determined with FITC-conjugated anti-fibrinogen antibodies. Platelet stimulation under stirred conditions led to a decrease of the platelet concentration as a consequence of platelet aggregation and a marked increase of the MV concentration. EDTA $(5 \mathrm{mmol} / \mathrm{s})$ or RGDS peptide $(1 \mathrm{mg} / \mathrm{ml})$ prevented the aggregation and the concomitant decrease of the platelet concentration and the formation of MV. In samples stimulated without stirring, neither decrease of platelet concentration nor shedding of MV occured, although the platelets exhibited maximal fibrinogen expression. Thus the aggregation of platelets is a prerequisite for the shedding of microvesicles from platelet membranes.

Med.-diagnost. Institut, Klinikum Karlsruhe, Germany. *Medizinische Biologie, Universität des Saarlandes, Homburg, Germany.

STUDIES ON PLATELET AGGREGATION IN WHOLE BLOOD OF VARIOUS SPECIES

\section{R. Westphal, B. Kaiser and E. Glusa}

Blood from different species (human, rat, rabbit) was anticoagulated $(1: 10)$ with citrate $(3.1 \%)$, heparin $(5 \mathrm{U} / \mathrm{ml})$ or hirudin $(200 \mathrm{ATU} / \mathrm{ml})$. Platelet aggregation in whole blood was induced by ADP, collagen, adrenaline, PAF, and arachidonic acid (AA). As inhibitors of platelet functions iloprost, acetyl salicylic acid (ASA) and daltroban were used

In citrated blood, collagen caused aggregation of platelets from all species, whereas ADP, AA and PAF aggregated only human platelets but not rat and rabbit platelets. In heparin anticoagulated blood collagen, ADP and AA induced aggregation of platelets from all species; PAF led to an aggregation in human and rabbit blood only. In hirudin - anticoagulated human, rabbit and rat blood, ADP, collagen and AA were potent aggregating agents. The PAF-induced aggregation was absent in rat blood. Adrenaline and 5-HT caused a negligible aggregation in human blood, however, the ADP-induced aggregation was potentiated by adrenaline and 5-HT in blood anticoagulated with citrate, heparin or hirudin.

The potency of the inhibitors was found to be agonist- and speciesdependet. The most potent inhibitor was iloprost with exception of collagen-induced aggregation in citrated rat blood. ASA was much less effective in inhibiting ADP-induced aggregation in whole blood from all species. The same is true of daltroban .

In conclusion, for experimental studies on platelet aggregation in whole blood the anticoagulants used, the aggregating agents and species differences have to be taken into account. Blood anticoagulated with heparin and especially hirudin should be preferred.

PLATELETS INHIBIT ELASTASE SECRETION IN NEUTROPHILS $M$. Dressel, S. Krause, H. Redlich, P. Spangenberg, S. Heptinstall, and W. Lösche

Elastase secreted form neutrophils is believed to play an important role in haemostatic processes. It has been shown that elastase is cytotoxic for endothelial cells, interferes with coagulation and influences platelet behaviour. Activated platelets are capable to adhere to neutrophils in a process that involves CD62 as well as fibrinogen bound to CD41a at the platelet surface. Recently, a stimulation of axygen radical generation in neutrophils by adherent platelets has been shown.

Upon in-vitro stimulation with agonists, such as the phorbol ester PMA, the ionophore A23187 or the chemotactic peptide FMLP, neutrophils secrete elastase into the medium which can be measured as proteolytic activity using a chromogenic substrate. Using these agonists the extent of elastase secretion was in the following order: 250nM PMA $<1 \mu \mathrm{M}$ FMLP $<24 \mu \mathrm{M}$ A23187 $<10 \mu \mathrm{M}$ FMLP. Addition of thrombin-activated and fixed platelets to neutrophils (ratio 50:1) resuited in a plateletneutrophil adhesion and an inhibition of elastase secretion. Interestingly, the extent of inhibition of enzyme secretion was depended on the extent of secretion observed in absence of platelets, i.e. highest inhibition at $10 \mu \mathrm{M}$ FMLP, lowest inhibition at $250 \mathrm{nM}$ PMA. By incubating neutrophil lysates with platelets it could be proved that platelets did not inhibit the elastase activity. Peptides that are known to interfere with the binding of fibrinogen to platelets and neutrophils and to partially reduce platelet-neutrophil adhesion (RGDS, GPRP) did not significantly change the inhibition of elastase secretion by platelets.

Our data indicate that platelets may inhibit elastase secretion from neutrophils in a contact-dependent way, which is in contrast to the previously reported stimulatory effect of platelets on neutrophil oxygen radical generation. Since there were no significant effects of peptides that interfere with fibrinogen binding we believe that the inhibition of neutrophil elastase secretion is mediated by $\mathrm{CD} 62$ rather than by fibrinogen exposed at the platelet surface.

Institute of Pathological Biochemistry, School of Medicine, POB 595, 99012 Erfurt, FGR, and "Department of Medicine, University Hospital, Nottingham NG7 2UH, UK 
THE ADHESION OF PLATELETS TO POLYMORPHONUCLEAR LEUKOCYTES (PMNL) - ON THE COUNTERRECEPTORS OF PLATELET SURFACE-BOUND FIBRINOGEN (FG) ON PMNL $P$. Spangenberg ${ }^{*}, H$. Redlich, R. Haferkorn, $W$. Lösche and $M$. Götzrath

We have recently shown that the adhesion of activated platelets to PMNL is mediated by CD62 and CD41a on the platelet surface. We have also shown that $F G$ acts as a bridging molecule between both cells. Here we present data on the counterreceptors of platelet surface-bound FG on the surface of PMNL.

In order to get information on the receptor(s) for the platelet bound $F G$ on PMNL we used peptides that contain sequences of the FG molecule (RGDS, echistatin and Gly-Pro-Arg-Pro (GPRP)). Some of these peptides are recognized by $\mathrm{CD} 11 \mathrm{c} / \mathrm{CD} 18$, a member of the LeuCAM-family, and by the leukocyte response integrin (LRI), the latter is complexed in the PMNL surface to an integrin-associated protein (IAP). We also used a monoclonal antibody (B6H12) which recognizes IAP and which inhibits the function of $|R|$

Adhesion experiments are performed at $4^{\circ} \mathrm{C}$ and at room temperature (RT), experiments at RT are associated with an increased adhesion. The adhesion that occurred at RT was significantly inhibited by DIDS, an anion channel blocking agent and an inhibitor of PMNL function, on the other hand the adhesion was increased by activating PMNL with PMA. These results may indicate that the adhesion partially depends on PMNL activation. At $4^{\circ} \mathrm{C}$ but not at RT RGDS and echistatin inhibited the adhesion, whereas GPRP and $\mathrm{B} 6 \mathrm{H} 12$ inhibited at RT but not at $4^{\circ} \mathrm{C}$. RGDS was found to potentiate the inhibitory effect of GPRP at RT. These data may indicate that the counterreceptors of platelet surface-bound FG on PMNL are both LRI and CD $11 \mathrm{c} / \mathrm{CD} 18$ and that the adhesion process has got an activation-dependent and -independent part. LRI is involved PMNL activation-independently (inhibition by RGDS and echistatin at $4^{\circ} \mathrm{C}$ ) as well as PMNL activation-dependently (inhibition by $\mathrm{B} 6 \mathrm{H} 12$ at $\mathrm{RT}$ ). $\mathrm{CD} 11 \mathrm{C} / \mathrm{CD} 18$ is involved PMNL activation-dependently only (inhibition by GPRP at RT).

Institute of Pathological Biochemistry, School of Medicine, POB 595, 99012 Erfurt, *present address: Fachhochschule Jena, Fachbereich Medizintechnik, POB 100314, 07703 Jena

PROTEIN KINASE C TRANSLOCATION IN URAEMIC PLATELETS. Ursula Frick, Klaus-Uwe Möritz, Ingelore Wiedenhöft, and Gerhard Frick

Proteinkinase $C$ (PKC) is found in high amounts in platelets. In response to diacylglycerol or phorbol esters the enzyme is redistributed from cytosolic to membrane fractions of the cell.

In patients with chronic uraemia and in a healthy control group PKC activities in cytosolic and particulate fractions were measured. In chronic uraemia we found a relatively high activity of PKC in membrane Fractions and a decrease in total activity of PKV about 30 per cent. The ratio between membrane bound and cytosolic PKC activity was 1.45 in contrast to 0.65 in healthy volunteers.

Results in literature indicate that stimulation of cell surface receptors of platelets by serotonine induce the redistribution of PKC. In the same patients we estimated serotonine uptake before ans after dialysis from platelets after collagen stimulation.

While the ability of platelets to take up serotonine in contrast to the control group is significantly reduced, serotonine release increased.

Our observations speak in favour of a translocation of PKC by crhonic uraemia. Increased serotonine release by these pa-

tients possibly leads to a self-stimulation of the cell surface receptors and to a translocation of PKC in the membrane. Decrease in total PKC activity is an indication for a fundamental irritation of platelet membrane.

Under this view serotonine receptor antagonists like ketanserin should be used to reduce the incidence of thrombosis in chronic uraemic patients.

Present Address: Inst. Clinical Chemistry, Ernst-Moritz-Arndt-University

FleischmannstraBe

17487 Greifswald, FRG
"ESSENTLAL" PIIOSPHOLIPIOS AND PLATELET ACTIVITY IN PATIENTS WITH HYPERLIPOPROTEINEMIA

Z.A.Gabbasov, I.Yu.Gavrilov, E.G.l'opov.

In this study we evaluated the influence of EPL (Lipostabil $N$, i.v.) on platelet funetional activity in 16 hyperlipoproteinemic men. The kinetics of aggregatc mean size changes during spontaneous and induced platelet aggregation was studied with a aggregation analyzer model $2201 \mathrm{~A}$ (Biola Ltd., Russia). Cholesterol/phospholipid ratio in platelet membranes was also measured. Platelet aggregation in palients suffering from IHD with a hyperlipoproteinemia of type If was increased with the severity of the disease and was greater than in healthy subjects. The difference was morc significant wben spontaneous platelet aggregation was measured or when small doses of the inducer were used. Inhibitory effect of Lipostabil $N$ on spontaneous and low dose induced platelet aggregation was ubserved: $3.66 \pm 0.67$ vs. $6.47 \pm 1.18(p=0.0045)$ for sporitaneous, $22.0 \pm 3.6$ vs. $57.4 \pm 22.8(p=0.026)$ for ADP-induced, $11.5 \pm 2.8$ vs. $28.5 \pm 11.5(p-0.02)$ for PAF-induced, $124 \pm 40$ vs. $152 \pm 52(p>0.1)$ for U46619-induced, (after and before treatment respectively). This effect was also observed 4 weeks afler treatment . The cholesterol per total phospholipid ratio ( $\mathrm{Ch} / \mathrm{P}$ ratio) is known to increase in platelets of hypercholesterolentic patients. In our study this ratio was $0.33 \pm 0,02 \mathrm{vs} .0 .24 \pm 0.01$ in nurmocholesterolemic controls $(p<0,01)$. After the Lipostabil $N$ infusions the ratio was decreased, $0.29 \pm 0.01$ vs. $0.33 \neq 0.02(p=0.0125)$ respectively after and belure treatment. We analyzed the possible relationship between $\mathrm{Ch} / \mathrm{PI}$ ratio and platelet aggregability and did not find correlation between absolute values of platelet aggregation and $\mathrm{Cl} / \mathrm{Pl}$ ratio. On the other hand we did find direct correlation between the relative changes in spontaneous platelet aggregation and $\mathrm{Ch} / \mathrm{Pl}$ ratio before and after treatment with Lipostabil $\mathrm{N}(\mathrm{r}=0.44$, $\mathrm{p} \times 0.01)$.

Present addres: Institute of Experimental Cardiology, Cardiology Researeh Center, 3rd Cherepkovskaya Str. 15a, Moscow, 121552, Russia

Bvaluation of a new ElISA (Thrombomatch) for the detection of platelet-reactive antibodies.

H. Bialleck ${ }^{1}$, P. Zumpe ${ }^{1}$, V. Lenhard ${ }^{2}$, E. Seifried ${ }^{1}$

1 Blutspendedienst Hessen des DRK, Frankfurt/Main 2 Biotest AG, Dreieich

The selection of compatible platelet donors for patients who are alloimmunized by multiple transfusions or by pregnancies is difficult and needs appropriate serological tests. The aim of this study was to evaluate a new ELISA (Thrombomatch by Biotest) for the detection of platelet-reactive antibodies. Sera from 119 patients were investigated: In the lymphocytotoxic test (LCT) 75 sera were negative and 43 were positive. In one serum antibodies were known against the human platelet antigen-1a (HPA-1a). The Thrombomatch was tested for its practicability, sensitivity and specifity by comparing it with three accepted methods for the detection of platelet antibodies: Platelet adhesion immunofluorescence test (PAIFT), modified Capture- $P^{R}\left(M^{R} P^{R}\right)$ and monoclonal antibody-specific immobilisation of platelet antigens (MAIPA). In addition, we correlated the absorbance units of positive test results of the MAIPA and the Thrombomatch.

The results of the Thrombomatch agreed well with the methods mentioned before; the MCP exhibited the lowest sensitivity.

In conclusion, the Thrombomatch is a very fast and easy to handle test system. It seems to be an appropriate screening-test for platelet-reactive and/or HLA-antibodies in refractory patients. The predictive clinical value of this assay has to be investigated in a prospective study. 
PLATELET AGgREgATION, PLATELET ADHESION TO SILICONIZED GLASS AND PLATELET-INDUCED THROMBIN GENERATION TIME (PITT) IN HEALTHY VOLUNTEERS AND IN MEDICAL PATIENTS TREATED WITH TICLOPIDINE. J.F.Schenk, P.Radziwon* , B.Boczkowska-Radziwon, M.C.Lalanne**, G.Schmidt, B.Scholz and H.K.Breddin

The efficacy of Ticlopidine in the prevention of thromboembolic events in high risk patients has been demonstrated in several clinical trials. The mode of action of Ticlopidine is up to date unclear but the blockage of an ADP-receptor on the platelet membrane is a convincing hypothesis. The aim of this study was to evaluate the ex vivo effect of an oral application of Ticlopidine on platelet adhesion and platelet induced thrombin generation time (PITT). This study was carried out as a randomized double blind cross-over placebo controlled study in 6 healthy valunteers (part $\|$ and as an open study in 15 patients (coronary heart disease, $n=8$, peripheral arterial disease, $n=7$ ) (part II). Platelet function and coagulation parameters were evaluated before drug adminstration and on days 3,5 and 7 in part $I$, additionally on days $10-14$ and 21-25 in part II. Ticlopidine $(250 \mathrm{mg})$ was applied orally twice daily. In healthy volunteers no prolongation of PITT values was observed either in the Ticlopidine or in the placebo group. A moderate and significant inhibition of spontaneous platelet aggregation was observed on day 3,5 and 7 in the Ticlopidine group. Platelet adhesion was markediy but not significantly reduced at day 5 and day 7 . Ticlopioine led to a significant inhibition of ADP-induced platelet aggregation on day 3. Part II: PITT values were moderately but not significantly prolonged on day 10 and on day 21. Using citrate PRP: Platelet adhesion was slightly reduced on day 3 and more evidently but not significantly on day 5 . Using Hirudin-PRP a slight and continuously increasing but not significant decrease of platelet adhesion occured up to the 21 th day. There was a strong and significant inhibition of ADP-induced and spontaneous aggregation on day $3,5,10$, and 21. This effect increased up to the 21 th day. aPTT. Fibrinogen and Factor VIII and F.VIII-ass.AG did not demonstrate relevant changes during the study period in both groups. Long term effects of Ticlopidine on platelet adhesion and on platelet induced thrombin generation if veryfied in further studies may be essential for the antithrombotic action of this agent.

Dept. of Internal Medicine, Division of Angiology, J.W.Goethe University T.Stern-Kai 7, 60590 Frankfurt/M, FRG

- Dept. of Haematology, Medical School, Bialystok, Poland

* Laboratoire d'Hematologie, Bordeaux, France

HEPARIN - ASSOCIATED THROMBOCYTOPENIA (HAT) TYPE II - STILI A DIAGNOSTIC AND THERAPEUTICAL PROBLEM IN CLINICAL PRACTICE

S. Kleinschmidt, U.T. Seyfert and E. Wenzel

There are several reports of Heparin - associated thrombocytopenia (HAT) Type II in the literature, but the cardinal symptom "thrombocytopenia" is rarely adequately considerd from a clinician's point of view. Serious and potential lethal complications such as pulmonary embolism, cerebral stroke or limb gangrene are often falsely regarded as "insufficient anticoagulation". Guided diagnosis and therapy are of vital importance for the patient's outcome. Based on our experience of 10 patients with HAT Type II treated in the intensive care unit, a diagnostic and therapeutic approach to the cardinal symptom "thrombocytopenia" by algorithms is presented. Recently developed heparin induced platelet activation assay (HIPAA) seems to be a high sensitive laboratory test. First therapeutical principle in case of presumed or/and diagnosed HAT is the cessation of all unfractionated and fractionated heparins. ORG 10172 (Orgaran), a low sulphated heparinoid with a cross reactivity (up to $10 \%$ ) to heparins, can be regarded as the most effective anticoagulant in patients with HAT Type II and is easy to manage especially under conditions of an intensive care unit.

Address: Unikliniken des Saarlandes, Klin. Haemostaseologie, Haus 75, 66421 Homburg, FRG
DEXTRAN SULPHATE LDL-APHERESIS IMPROVES (IN-VTVO) PLATELET FUNCTION

H.Sinzinger, J.A.Flores, Ch.Pirich, Judith Bednar, Susanne Granegger

LDL-apheresis is the treatment of choice in patients with severe familial hyperlipoproteinemia (FH). As the role of blood lipids (e.g. cholesterol) on platelet function is still under debate, we wondered whether regular apheresis treatment may change in-vivo platelet function.

8 patients with $\mathrm{FH}(7 \mathrm{~m}, 1 \mathrm{f}, 27-60$ years) underwent platelet labeling with $100 \mu \mathrm{Ci}{ }^{311}$ In-oxine before and 6 months after regular LDL-apheresis (plasma exchange $3000 \mathrm{ml}$; $\mathrm{CH}$ before therapy $504 \pm 36 \mathrm{mg} / \mathrm{dl}$, before LDL-apheresis $336 \pm 22 \mathrm{mg} / \mathrm{dl}$, after $122 \pm 19 \mathrm{mg} / \mathrm{dl}$ ). Platelet labeling improved $(89,0 \pm 2,4 \%$ vs $38,2 \pm 5,9 \%)$ as did recovery $(60,7 \pm 2,6 \%$ vs $19,7 \pm 4,5 \%$ ). Spleen - liver ratio did not change $(0,77 \pm 0,1$ vs $0,73 \pm$ $0,1)$, while platelet survival improved significantly from $106,5 \pm 24,9$ hours to $137,5 \pm 17,0$ hours.

These findings indicate that normalisation of excessively elevated blood lipids results in an improved labeling behavior, cellular viability and in-vivo haemostasis.

This benefit at least in part may underly the almost inmmediate regressional changes seen in these patients.

*Present address: Wilhelm Auerswald Atheroscierosis Research Group (ASF) Vienna, Nadlergasse 1, A-1090 Vienna, Austria.

146

Influence of Halothane on Platelet Activation

N.Kutz ${ }^{1}$. D. Frotlich ${ }^{1}, M$, Koksch $^{2}$, G. Rothe ${ }^{2}$, G. Schmitz ${ }^{2}$.K. Taeger ${ }^{1}$

Klinik fur Anästhesiologie der Universitat Regensburg, Dir.: Prof Drmed $K$ Taeger

2 institut tor Winische Chemie der Universität Regensburg. Dir.: Prof. Dr.med. G. Schmitz

introduction:

The volatile anesthetic halothane influences platelet activity. The basic pathomechanisms are unknown. Highly sensitive markers of activation are the epitopes CD $42 \mathrm{~b}$ und $C D 62$ on the platelet surface. We studied the effects of different hathe concentrations on these parameters by flow surface. We studied the effects of different halothane concentrations on these parameters by flow cytometry. During activation $C D 42 b$ is shifted to the platelet core, whereas $C D 62$ is increasingly presented on the platelet surface (Kieffer ef al, 1992). Due to these opposed responses relative
changes of the receptor density caused by aggregates or thrombocyte volume alterations can be excluded.

Methods:

Thrombocytes from 7 healthy donors were incubated for $30 \mathrm{~min}$ in 0.35 vol\%, 0.7 vol\%, 1.4 vol\% halothane/air midures. Air was used for reference. The fluorescence intensities of 5000 platelets per aliquot were analysed by flow cytometry after staining with directly conjugated monoclonal cantiaining a defined number of anti-mouse-lg binding sites. All steps of preparation were performed inside an airtight glove-box in a halothane-equilibated athmosphere.

Results:

1. Halothane treated thrombocytes showed a significant $(p<0.05$ ) increase in $C D 62$ an the surface membrane from a mean of 460 antigenesiptatelet at air to 1125 antigenes/phatel at 1.4 vol\% malothane.

2. With halothane there was a significant $(0<0.05)$ reduction of $C D 42 \mathrm{~b}$ receptor density from 34725 antigenes/thrombocyte (mean) at air to 27600 antigenes/thrombocyte at 1.4 vol\% haichane.

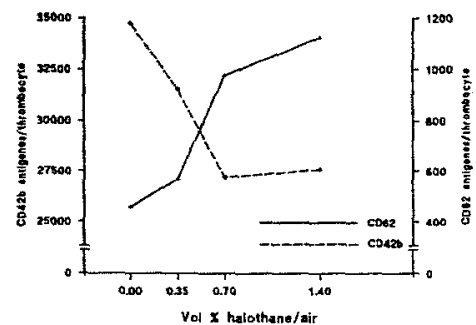

Fig. 1 Infiuence of hatothane on the density of epitopes $C D 42 \mathrm{~b}$ and $C D$ 62 on thrombocytes

Conclusions:

Haicthane leads to a decrease in $C D$ $42 \mathrm{~b}$ density and an increase in the number of $C D 62$ epitopes in vitro This indicates ptatelet activation at clinically relevant concentrations of hatothane. Reference: Kiefter $N$. et al. Dynamic Redistribution of Major Platelet Surface Receptors after Contact-induced Platelet Activation and Spreading. AmJPathoi 140:5773 (1992) 
Heparin associated Thrombocytopenia (HAT 2 ): AT III Concentrate Administration as Therapy of Choice for Prophylaxis and Treatment of Thromboembolic as Therapy of Choice for

R. Egbring, U. Peters, R. Funck, R. Seilz, M. Maasberb, H. Blanke, A. Greinacher, K. Andrassy

Heparin associated thrombocytopenia is an uncommon complication during anticoagulant treatment with heparin as it is indicated in paltents wilh myocardial infarction, atterial or venous thrombosis as well as in DIC and during and after thrombolytic therapy.

HAT $_{2}$ may be complicaled by bleeding, micro and macro vessel thrombosis as well as by an increased throver of cotting factors and platelets (DiC). Greinacher developed a so called HipA lest system, in

We woutd like to present 5 pts. with HAT ${ }_{2}$ with different bleeding and thrombotic complications of DIC. Organon Heparinoid ocsured as stown in the following table.

\begin{tabular}{|c|c|c|c|c|c|c|}
\hline $\begin{array}{l}\text { Disorder } \\
\text { of patients }\end{array}$ & $\begin{array}{l}\text { day } \\
\text { adn } \\
\text { Haw }\end{array}$ & $\begin{array}{l}\text { of heparin } \\
\text { nistration } \\
\text { i. inany }\end{array}$ & $\begin{array}{l}\text { lowest platefet } \\
\text { coumt }\left[x 10^{6 / m} / \mathrm{m}\right] \\
\text { HMWH-1 }\end{array}$ & et $\begin{array}{l}\text { AT III } \\
\text { administration }\end{array}$ & $\begin{array}{l}\text { HipA- } \\
\text { test }\end{array}$ & $\begin{array}{l}\text { Complications during } \\
\text { hepanin administration }\end{array}$ \\
\hline $\begin{array}{l}\text { deep } \\
\text { vein } \\
\text { thrombosis }\end{array}$ & 14 & $\stackrel{14}{14}$ & 30 & $\begin{array}{c}+ \\
\text { for prophylaxis, } \\
\text { without heparin }\end{array}$ & $+\ldots$ & $\begin{array}{l}\text { venous } \\
\text { thrombosis }\end{array}$ \\
\hline $\begin{array}{l}\text { Myocardia: } \\
\text { infarction }\end{array}$ & 4 & $\stackrel{2}{2}$ & 43 & $\begin{array}{c}+ \\
\text { therapectic, } \\
\text { without heparin }\end{array}$ & +++ & $\begin{array}{l}\text { iliac artery } \\
\text { thrombosis }\end{array}$ \\
\hline $\begin{array}{l}\text { Oic following } \\
\text { surgery }\end{array}$ & 19 & - & tin & $\begin{array}{l}+ \\
\text { therapettic, } \\
\text { unfortunately con- } \\
\text { tirused with heparin }\end{array}$ & +++ & $\begin{array}{l}\text { Dleeding } \\
\text { (retroperitoneal) }\end{array}$ \\
\hline $\begin{array}{l}\text { Myocardial } \\
\text { infarction }\end{array}$ & 20 & - & 73 & - & ++ & $\begin{array}{l}\text { DIC. ARF, } \\
\text { erythema, } \\
\text { skin necrosis }\end{array}$ \\
\hline $\begin{array}{l}\text { traumatic } \\
\text { npture of } \\
\text { m. quadri- } \\
\text { ceps } \\
\text { (immobitization }\end{array}$ & 9 & - & Or & $\begin{array}{c}\text { tedic, } \\
\text { therapeustic, } \\
\text { continued with } \\
\text { Organan heparinoid }\end{array}$ & +++ & $\begin{array}{l}\text { bleeding, plegmasia } \\
\text { coerulea dolens, } \\
\text { thrombosis of } \\
\text { v. iliaca ext. v. femo } \\
\text { ralis, v. poplitea }\end{array}$ \\
\hline
\end{tabular}

Discussion; Antithrombin HIl replacement was performed as articoagulant in combination with high and low molecatiar weight heparin and alone. In case of $H A T_{2}$ it will be a successful therapy. It may be the therapy Which platelet aggregation is measured in
2 different concentrations of heparin. Spontaneous improvement, lethal outcome and a successfully performed antithrombin III concentrat

\section{9}

ACQUIRED MACROTHROMBOCYTOPATHY WTH ABNORMAL GLYCOPROTEIN IB

C.M. Kirchmaier, K. Becker-Hagendorff, D. Westrup, M. Meyer*, E. Morgenstern**, H.K. Breddin

A 46 years old patient developed within two months a severe haemormagic diathesis. The patient himself and his family had no bleeding history in the past. The patient suffered on a liver cirhosis and was admitted in our hospital because of a renal insufficiency.

Cells of an unknown tumor were detected in the ascites fluid. During hospitalisation the bleeding symptoms increased and the patient died two months after admission from a gastrointestinal bleeding. The marked bleeding defect cannot be explained by slight changes of the clotting factors caused by liver cirthosis.

Bleeding time was markedly prolonged (Simplate $I>30 \mathrm{~min}$ ) and a platelet function defect similar to a Bemand-Soulier Syndrome was detected with increased platelet volume $(13,7 \mathrm{fl})$ and inhibited Ristocetin $(1 \mathrm{mg} / \mathrm{ml})$ and thrombin $(0,1 \mathrm{U} / \mathrm{ml})$ induced platelet aggregation.

Platelet adhesion on human endothelial matrix (ECM) was markedly inhibited and on siliconized glass only slightly reduced.

In gelelectrophoresis the glycoprotein lb content was markedly reduced, the electrophoresis pattem was not comparable because enzymatic digestion by elastase caused by an increased number of leucocytes $(22.000 / \mu 1)$ may have occured.

In electron microscopy giant platelets with peripheral microtubules comparable with the typical findings in platelets from Bernard-Soulier patients were detected.

Center of Internal Medicine, Dept. of Angiology, J.W.G.-University, Theodor Stern Kai 7, 60590 Frankfurt/M., *Medical Academy, Erfurt, *Dept. of Med. Biology, Homburg/Saar,

\section{LEUKOCYTE-DEPLETION BY FILTRATION REMOVES PLATELET FACTOR 4 BUT NOT B-THROMBOGLOBULIN FROM PLATELET CONCENTRATES}

M.Riewald, A. Putzo, V. Weisbach, G. Himmelreich, R. Eckstein, and $\mathrm{H}$. Riess

Storage of platelet concentrates (PCs) and leukocyte-depletion (LD) are accepted to provide platelet support and to prevent alloimmunisation, respectively. Transfusion PCs stored for several days exposes the recipient to high levels of products released by platelets, such as heparin-neutralising platelet factor 4 ( $\mathrm{PF} 4)$ or Bthromboglobulin (BTG). The effects of these substances on hemostasis are speculative but may be harmful, e.g. in hypercoagulable states.

We investigated the effect of LD by a polyester filter (PL-50, Pall, Dreieich, Germany) of 9 PCs stored for 5 days on PC plasma levels of PF4 and BTG.

During storage PF4 increased ( $\mathrm{p}<0.01)$ from 1020 [range:260-7000] $\mathrm{IU} / \mathrm{ml}$ to $6100[4000-8370] \mathrm{IU} / \mathrm{ml}$ and BTG from 2450 [835-15500] $\mathrm{IU} / \mathrm{ml}$ to 11500 [5950-19000] $\mathrm{\Pi U} / \mathrm{ml}$. Filtation of the stored PCs not only resulted in a more than $95 \%$ reduction in leukocytes but also in a significant $(p<0.01)$ reduction of PF4 to 900 [450-1840] $\mathrm{IU} / \mathrm{ml}$, whereas $B T G$ remained unchanged.

Using fractions of platelet-free PF4-enriched plasma $(28000 \mathrm{IU} / \mathrm{ml})$ more than $80 \%$ of PF4 in $200 \mathrm{ml}$ are cleared during one filter passage without reaching the clearance capability of the filter.

We conclude that LD by filtration reduces the amount of free PF4 but not BTG - to the pre-storage level. The clinical impact of this phenomenon has to be investigated. Further studies on the clearance of other plasma components during LD are warranted.

Universitätsklinikum Rudolf Virchow, Department of Internal Medicine, Spandauer Damm 130, 14050 Berlin

\section{HEPARIN-ASSOCIATED THROMBOCYTOPENIA: THE EFFECTS OF VARIOUS INTRAVENOUS IGG PREPARATIONS ON ANTIBODY MEDIATED PLATELET ACTIVATION.}

A POSSIBLE NEW INDICATION FOR HIGH DOSE I.V. IgG.

A. Greinacher ${ }^{\dagger}$, U. Liebenhotf ${ }^{2}$, V. Kiefel ${ }^{1}$, P. Presek ${ }^{2}$, and C. Mueller-Eckhardt ${ }^{1}$

Heparin-associated thrombocytopenia (HAT) is caused by an antibody which, in the presence of polysulfated oligosaccharides, activates platelets via the Fc-receptor. The antigen is formed by a releasable platelet protein, in many cases PF4 complexed to heparin. As the role of $\mathrm{gp} \mathrm{IIb/lila} \mathrm{in}$ platelet activation in HAT is controversial, we investigated platelet activation by the HAT antibody using normal platelets and platelets from a patient with Glanzmann's thrombasthenia (GT) lacking gp lib/llia. Heparin and sera from patients with HAT stimulated GT platelets in the same manner as determined by ${ }^{14} \mathrm{C}$-serotonin release and by changes in phosphorylation of p20 and p47. Platelet activation could be inhibited by an anti FCRII monocional antibody (IV.3, Fab), and by Fc-fragments, but not by $F\left(a b^{\prime}\right)_{2}$ fragments of human lgG and a $5 \mathrm{~S} \mathrm{IgG} \mathrm{preparation.} \mathrm{The} \mathrm{effect} \mathrm{of} \mathrm{four}$ different, commercially available preparations of intact i.v. IgG on the platelet activating effect of six HAT sera was investigated by ${ }^{14} \mathrm{C}$-serotonin release. The inhibitory effect was strongly dependent upon the manufacturing process. At a concentration of $20 \mathrm{mg} / \mathrm{ml}$ only lgG that had been subjected to low $\mathrm{pH}$ and traces of pepsin sufficiently inhibited platelet activation (index:0.62). $\lg \mathrm{G}$ treated with polyethylenglycol (i:0.35) or sulfitolysis (i:0.32) was less effective, whereas beta propiolactone-treated IgG almost completely lost the ability to inhibit platelet activation by the HAT antibody (i:0.23).

We conclude that inhibition of gp llb/llla - fibrinogen interaction is insufficient for preventing platelet activation in HAT. This is, however, possible by high dose i.v. lgG, whereby inhibition of FcRll on platelets strongly depends upon the process by which the i.v. IgG preparation was manufactured.

1 Institut für Klinische Immunologie and Transfusionsmedizin.

2 Rudolf-Buchheim-Institut für Pharmakologie, Justus Liebig Universität, Giessen. 
151

Standardized Flow Cytometric Characterization of Platelet Dysfunction

\section{Koksch, G. Rothe, G. Schmitz}

Institute of Clinical Chemistry, Central Laboratory and Blood Bank, University of Regensburg, Germany

The diagnosis and quantitation of platelet hyper- or hyporeagibility is an important aspect in thrombotic and pre-thrombotic syndromes, atherosclerotic and inflammatory vascular diseases, diabetes mellitus, hematologic disorders, and following contact activation during extracorporal circulation. Currently, functional platelet tests (e.g. aggregometry) are not widely accepted as a diagnostic tool due to high consumption of time and material and a low sensitivity.

Flow cytometric analysis of surface antigen densities of platelet specific antigens (CD41a $\left.{ }^{1}, C D_{2} \mathrm{~b}^{2}{ }^{2} \mathrm{CD} 62, \mathrm{CD} 63\right)$ allowed new insights in mechanisms of platelet activation. This method is also suited for very low platelet counts. The goal of this study was the development of a standardized protocol with an antibody-independent calibration for the ex vivo measurement of surface antigen densities and for the functional characterization after in vitro stimulation.

We tested a fixation-free stabilization (enzyme inhibitors, chelators), a gentle sample preparation (centrifugation vs. Whole blood analysis) and the calibration with beads defined in their number of binding-sites for antimouse-lgG. Cells were stimulated in vitro with ADP, collagen and ristocetin. For each analysis 20-200 $\mu \mathrm{l}$ of platelet-rich plasma is used, thrombocytopenia with less than 10.000 platelets/ $\mu$ is no limitation to the method. If the analysis is performed within 2 hours after blood drawing, no addition of stabilizing agents is required (stabilization of the expressed antigens for $48-72 \mathrm{~h}$ with $3 \mathrm{mM}$ Theophyllin and $30 \mathrm{mM} \mathrm{MgCl} 2$ ). ADP. induced differences in surface antigen expression were more sensitive in discriminating between healthy controls and patients with pre-thrombotic syndrome and/or neoplasia than ex vivo antigen densities.

1 Nomura, S. et al. Cytometry 13 (1992) 621-29

2Polanowska-Grabowska, R. et al. Br.J.Haematol. 82 (1992) 715-20

${ }^{3}$ Tschöpe, D. et al. Cytometry 11 (1990) 652-656

THROMBOXANE A POTENTIATES PROLIFERATION OF VASCULAR SMOOTH MUSCLE CELLS BY PLATELET-DERIVED GROWTH FACTOR T. Großer, E. Bretschneider ${ }^{+}$, T. Morinelli ${ }^{*}$, E. Glusa ${ }^{+}$, K. Schrör

Platelet-derived growth factors (PDGF) are vasoconstrictors and important peptide mediators of smooth muscle cell (SMC) proliferation. This study investigates the modulation of PDGF-mediated proliferation of SMC by a thromboxane $A_{2}$-mimetic (1-BOP).

SMC from bovine coronary arteries were incubated for $24 \mathrm{~h}$ with PDGF-AA, $A B$ and $B B(20 \mathrm{ng} / \mathrm{ml})$ in serum-free medium. $\left[{ }^{3} \mathrm{H}\right]$-thymidinc-incorporation was measured as an index of DNA synthesis. PDGF-AA, AB and BB stimulated $\left[{ }^{3} \mathrm{H}\right]-$ thymidine-incorporation (mean from triplicate measurements in 2 separate assays) from 93 to 142, 354 and $484 \mathrm{cpm} / \mu \mathrm{g}$ protein. Coincubation of PDGF-BB with the thromboxane mimetic I-BOP $(100 \mathrm{nM})$ stimulated this value to $724 \mathrm{cpm} / \mathrm{\mu g}$ protein. Similar, though less pronounced stimulation was also seen with the PDGF$\mathrm{AA}$ and $-\mathrm{AB}$ isoforms. The thromboxane receptor antagonist SQ 29,548 reduced these potentiations to nearly control levels in all experiments. There were no direct effects of I-BOP or SQ 29,548 on this parameter in the absence of PDGF and no measurable $\mathrm{TXA}_{2}$ formation ( $<10 \mathrm{ng} / \mathrm{ml}$ in $24 \mathrm{~h}$ ) by SMC. Radioligand binding studies demonstrated high-sffinity binding sites for $\left[{ }^{3} \mathrm{H}\right]$-SQ $29,548\left(\mathrm{~K}_{\mathrm{d}} ; 1.6 \mathrm{nM}\right)$ equivalent to 2,300 thromboxane receptors per SMC.

These data demonstrate specific, receptor-mediated potentiation of proliferative actions of PDGF isoforms on vascular SMC by TXA, in the absence of significant $\mathrm{TXA}_{2}$ production by SMC and the presence of specific $\mathrm{TXA}_{2}$ receptors. This suggests an important role for mediators released from adhering platelets for SMC growth stimulation at sites of a vessel injury.

Institut für Pharmakologie, Heinrich-Heine-Universität Düsseldorf, Moorenstr. 5, 40225 Düsseldorf, "Dept. Clin. Pharmacology, MUSC Charleston, SC, USA, ${ }^{+}$Forschungszentrum für Vaskuläre Biologie und Medizin Erfurt
153

ANALYSIS OF PLATELET COUNTS BY MULTIPARAMETER FLOW CYTOMETRY USING SPECIFIC PLATELET ANTIBODIES (anti-CD41a) AND LATEX BEADS

A. v. Ruecker, A. Theisen, R. Dickerhoff, and F. Bidlingmaier

The correct enumeration of platelets is still an elusive matter. This is partiy due to the fact that most commercial instruments and methods used for platelet counting do not discriminate platelets from other particles, air bubbles, electronic noise, etc. that cause similar size-signals. Furthermore coincidence counts (erythrocyte/platelet or platelet/platelet) may be a critical factor especially in aperture-impedance instruments. Manual methods employing a counting chamber are still frequently used in routine laboratories to verify low automated platelet counts (10 to $\left.50 \times 10^{9} / \mathrm{L}\right)$ despite obvious statistical and subjective drawbacks.

Our experiments were conducted with a multiparameter flow cytometer using laser optics and a maximal particle flow rate of $1000 / \mathrm{s}$ to exclude coincidence. The lack of specificity to identify platelets was overcome by applying the platelet specific anti-CD41a-FITC monoclonal antibody. To calculate the precise platelet count, a defined concentration of FITC-labelled latex-beads in whole blood was established after treatment with platelet specific ansi-CD41 a-FITC antibodies. These FITC-beads had distinctly different forward and side scatter characteristics than the labelled platelets but were similar in FITC fluorescence. By means of a FITC-fluorescence threshold set in the flow cytometer the ratio of FITC-labelled beads and platelets was determined and, since the bead concentration is known, the platelet concentration could be easily calculated from this ratio.

Our results demonstrate that optimal bead to platelet ratios may range from 0.2 to 5. Statistical difficulties can arise above or below these levels. Dilution experiments conducted at these levels were highly linear for both platelets and beads. Precision measurements at 7 different platelet concentrations had an average coefficient of variation of only $2.4 \%(\mathrm{n}=5)$. Comparability studies with counting chambers, automated blood counters (Technicon H-1, Sysmex E-5000) and a platelet/erythrocyte ratio counting method (ICSH proposal) were performed. At normal platelet concentrations $\left(150-400 \times 10^{9} / \mathrm{L}\right)$ platelet counts of all methods agreed satisfactorily $(\mathrm{n}=20$; coefficient of correlation $\mathrm{r}=0.89-0.99$ ). At low platelet concentrations $\left(10-30 \times 10^{9} / \mathrm{L}\right)$ our new method correlated satisfactorily $(n=20)$ only with the counting chamber $(r=0.89)$ and ICSH-ratio method $(r=0.82)$. Two cases of EDTA-induced pseudo-thrombocytopenia were detected by our method. In conclusion our findings stress the necessity to confirm low platelet counts determined by automated blood counters and suggest that the above method may be the easiest way to do so.

Institut für Klin. Biochemie, University of Bonn, D-53105 Bonn, Germany

\section{COMPARATIVE STUDIES ON HUMAN PLATELET ACTIVATION AND ENDOTHELIUM-DEPENDENT VASCULAR RELAXATION BY THROMBIN RECEPTOR ACTIVATING PEPTIDES (TRAP) E.Glusa}

Thrombin activates its receptor by cleavage of a peptide from the extraceliular $\mathrm{N}$-terminus. Synthetic peptides corresponding to the first 6 and 14 amino acids (AA) of the newly generated $N$-terminus are able to activate directly the thrombin receptor and to mimic the effects of thombin. Some experimental studies have shown that the thrombin receptor activating peptide (TRAP) with 6 AA (TRAP-6) was more potent than TRAP with 14 AA (TRAP-14). In the present investigation the effects of both peptides on human platelets and porcine pulmonary arteries with intact endothelium were compared. TRAP- 6 and TRAP-14 at micromolar concentrations caused platelet aggregation in Tyrode's solution, citrated and hirudinized plasma; increase in cytosolic calcium ions, and thromboxane formation which was more pronounced in citrated plasma than in hirudinized plasma. There were no significant differences in potency of the two peptides. In porcine pulmonary arteries with intact endothelium, TRAP-6 and TRAP-14 induced an endothelium-dependent relaxation which was absent after mechanical removal of endothelium or pretreatment with nitro-L-arginine which blocks the synthesis of endothelium-derived nitric oxide. The concentrations of the peptides necessary for relaxation were in the same range as those for activation of platelets. In comparison to thrombin (acting at nanomolar concentrations), TRAP-6 and TRAP-14 were less potent by more than three orders of magnitude.

Institute of Pharmacology and Toxicology, Medical School Erfurt, Nordhäuser Str. 74, D-99089 Erfurt 


\section{PLATELET RESPONSE TO VASCULAR SURGERY - EFFECT OF ACETYLSALICYLIC ACID AND HEPARIN THERAPY \\ C.B. Reininger ${ }^{1}$, A.J. Reininger ${ }^{2}$, B. Steckmeier ${ }^{1}$, A. Greinacher ${ }^{3}$, R. Lasser ${ }^{4}$, L. Schweiberer ${ }^{1}$}

Enhanced plateiet reactivity has been postulated for patients with peripheral arterial disease (PAD). The effect of acetylsalicylic acid (ASS), heparin and vascular surgery on platelet reactivity in such patients was examined using the Stagnation Point Flow Adhesio- Aggregometer (SPAA).

The SPAA provides well defined flow conditions whereby platelet microthrombus formation can be directly observed and measured continuousty. Mathematical evaluation of resulting growth curves renders the constants reflecting platelet adhesivity and aggregability, Kpw (\%) and Kpp (\%), respectively. The adhesivity and aggregability of the platelets of 44 PAD patients were quantitated perioperatively. Preoperative values were compared to those of 26 controls (ASS and heparin free). Plasma fibrinogen concentration and platelet count were also determined. The heparin-induced platelet activation (HIPA) assay for detection of heparin-associated thrombocytopenia (HAT) antibodies was performed for 25 patients.

Baseline values of SPAA-measured platelet reactivity $(p<0.01)$ and plasma fibrinogen $(p<0.05)$ were higher for patients as compared to controls and increased further after surgery. Maximum platelet activation and fibrinogen levels (2nd postoperative day) were observed concomitant to application of unfractionated heparin and coincided with a marked drop in platelet count (> than $50 \%$ ). The HIPA test verified HAT antibodies in $3 / 25(12 \%)$ patients, two of which suffered postoperative thrombosis. In 5/25 (20\%) the presence of HAT antibodies could not be ruled out definitively.

In the presence of pathologically enhanced preoperative platelet function vascular surgery results in a further increase in platelet reactivity in spite of ASS therapy. Heparin may have promoted the observed platelet activation, as indicated by the concomitant decrease in platelet count and coincidence of postoperative thrombosis with HAT antibody detection.

${ }^{1}$ Chirurg. Klinik Innenstadt, Ludwig-Maximilians-Universität, NuBbaumstr. 20 80336 München, Germany, ${ }^{2}$ Anatomisches Inst., Techn. Universität München, 3 Inst. für Klin. Immunologie und Transfusionsmed., Justus-LiebigUniversität Giessen, ${ }^{4}$ GSF-Forschungszentrum, MEDIS Inst. Neuherberg

\section{Contact Phase}

156

\section{SPECIFIC ADSORPTION OF HEPARIN TO EXTRACORPO-REAL DEVICES \\ J. Harenberg. R. Malsch. M. Schäfer, and Ch. Giese}

Extracorporeal devices such as artificial kidney and oxygenators all rely on the patient being heparinized to prevent blood clotting in the device. The need to fully heparinize patients undergoing extracorporeal circulation often leads to haemorrhagic complications. Bonding of heparin to the device is one of the approaches to solve this problem. Here we describe the adsorption of heparin to a polysulfon surface and the anticoagulant and antithrombotic properties.

Heparin was adsorbed to the polysulfon surface of a hemodialysis filter (diaflo $F$ 40, Fresenius) and the flexible support by electrostatic forces. The surface was first provided with positive ionic groups using different amounts of $\mathrm{N}$-cetylpyridinium chloride. The filter was incubated with $0.02 \%$ to $1.0 \%$ heparin in aqueous solution. Crosslinking of heparin was achieved by addition of 0.1 to $0.5 \%$ glu-taraldehyde solution.

Native blood from healthy volunteers was filled into the heparin coated hemodialysis filter and flexible supports and was allowed to circulate with a speed of $100 \mathrm{ml} / \mathrm{min}$ for $3 \mathrm{hrs}$. Thrombin generation (TAT-complexes) was not inhibited in control experiments if one of the reactants was omitted or if the concentration of heparin was to low. Under optimized conditions heparin binding to the polysulfon surface was more effective in inhibiting thrombin formation for 3 hrs despite lower anti-factor Xa levels as compared to a bolus of the same dose of heparin.

A method of heparin adsorption to polysulfon surfaces is presented, which is more effective in inhibiting thrombin formation despite lower anticoagulant effects than conventional heparinization of blood for anticoagulation of an extracorporeal device.

Supported by Deutsche Forschungsgemeinschaft (Ha 1164/3-2) 1. Medizinische Klinik, Fakultät Klinische Medizin Mannheim, Theodor Kutzer Ufer, 68167 Mannheim
157

\section{F XII DEFICIENCY AMONG 126 PATIENTS WITH CORONARY} HEART DISEASE (CHD) AWAITING CARDIAC SURGERY

W.-M. Halbmayer, A. Haushofer, J. Radek, R. Schön, M. Deutsch* and M. Fischer.

In the literature several case reports of myocardial infarction in patients with F XII deficiency are described. Therefore, F XII activity was measured in the plasmas of 426 consecutive patients with coronary heart disease (CHD) awaiting cardiac surgery (aorto-coronary vein graft bypassing). Among them 44 patients $(10.3$ \%) could be detected as moderatly deficient of F XII. The prevalence of F XII deficiency among patients with CHD was significantly higher ( $p<0.0001$ ) when compared with the incidence of $F$ XII deficiency among 300 healthy blood donors $(2.3 \%)$, which has been reported previously. When compared with CHD patients without F XII deficiency, patients with F XII deficiency showed no different incidence of elevated plasma levels of fibrinogen, lipoprotein(a) and no different incidence of high blood pressure, cigarette smoking or positive family history of thromboembolism.

Central Laboratory and * 1 st Department of Surgery of the General Hospital Vienna-Lainz, Wolkersbergenstraße 1, A-1130 Vienna, Austria. 
THE REACTION OF THE CONTACT SYSTEM DURING THE OPERATIVE THERAPY OF COLORECTAL CARCINOMAS W. Heller, H.P. Wendel*

The aim of the present study was to gain insight into the state and the reaction of the contact (kallikrein-kinin) system through the observation of individual parameters of this system during the operative therapy of 40 patients with colorectal carcinoma (1). As a control group (2) we studied a group of patients who had to undergo surgery for benign tumors of the same severity.

With determination of prekallikrein, kallikrein-like actjvity and $\mathrm{C} 1$ inhibitor, the main inhibitor of kallikrein and $\alpha$ - and $\beta$-factor XIla the parameters of the contact phase were investigated. The course of the prekallikrein levels were characterized by a drop that continued right in the postoperative phase with hesitant rise again. The significant lower activity with the existing formation metastases suggest a specific connection between prekallikrein and tumor. It must be assumed that with the generalization of the illness the pathomechanism leads to an activation of the kallikrein-kinin system. As a measure of the $\alpha 2$-macroglobulinkallikrein complexes present the kallikrein-like activity confirmed the activation that occurred in the operative phase. In this situation the function of kallikrein inhibition has been taken over to a great extend by the $\mathrm{C} 1$-inhibitor. The $\mathrm{Cl}$-inhibitor behaved in accordance with the acute phase proteins. The determination of kallikrein inhibition yielded evidence of constantly higher functional activity that had exceeded the normal range at the patients' discharge. In malignant processes it has been proven that the tumor tissue is able to form a kind of camouflage cover via a coating of the cell membrane with $\mathrm{Cl}$-inhibitor thus rendering the tumor cell safe from attack by the humoral defence system. A further inhibitory function of $\mathrm{C1}$ inhibitor was investigated with the determination of the $\beta$-factor XIla inhibition.

*Present address: Dept. of Thoracic and Cardiovascular Surg., Univ. of Tuebingen,

Calwerstr. 7, D-72076 Tuebingen, Germany

Soluble fibin and fibrin degradation Products in Patients after CardioPULMONARY BYPASS SURgery - EVALUATION OFA NOVEL D-Dimer ASSAY J.U. Wieding, S. Hagenlocher, J. Rathgeber, D. Zenker and M. Unterhalt

The activation of hemostasis in patients undergoing cardiopulmonary bypass surgery leads to an increased fibrinogen/fibrin turnover. In this study fibrin(ogen) derivatives and other markers of the regulation of hemostasis as well as several cytokines were measured in 14 patients; in each of them a total of 8 blood samples was taken preoperatively as well as up to $4 / 6$ days after surgery.

An increase in IL-6 was already measured during surgery; a peak of $90 \mathrm{pg} / \mathrm{m}$ was reached $3 \mathrm{~h}$ after the end of surgery. The values declined slightily during the next $6 \mathrm{~h}$ and reached normal levels $(<1 \mathrm{pg} / \mathrm{ml})$ after $72 \mathrm{~h}$.

Soluble fibrin (SF), D-dimers and other fibrin degradation products all indicated fibrin formation and lysis. SF was measured by means of the protamine sulfate turbidimetry that detects the aggregability of SF, which is thought to correlate with the patient's risk for intravascular fibrin deposits. Whereas soluble fibrin only slightly increased during surgery, SF-values significantly increased $18 \mathrm{~h}$ later and peaked after further $48 \mathrm{~h}$. Normal values were reached at day 6 postoperatively. D-dimers were elevated intra-/postoperatively ( $1.6 \mathrm{bis} 1.4 \mathrm{ug} / \mathrm{ml})$ and moderately decreased within the next $48 \mathrm{~h}$; following the SF fevels with a delay of $20 \mathrm{~h}$, the D-dimers increased again to considerably higher levels ( $3.5 \mathrm{ug} / \mathrm{m} /$ on day 4 ).

D-dimer levels were comparatively measured by 3 different techniques, a novel membrane-flow-through immunoassay (MIA) from Fa. Immuno GmbH (=NycoCard ${ }^{\mathrm{TM}}$ ), an established latex agglutination test (LAT) and an enzymelinked immunosorbent assay (ELISA). The results from MIA and LAT correlated with $r=0.94$, MIA and ELISA with $r=0.81$ and results from LAT and ELISA with $r=0.79 \quad(n=110,150,115$, resp. $)$. Notably, at levels exceeding $8 \mathrm{ug} / \mathrm{ml}$ the ELISA-values did not correlate as well with values from LAT or MIA. Taken together the novel MiA-based NycoCard ${ }^{\mathrm{TM}}$ D-Dimer test demonstrated an excellent practicability and at least the same sensitivity as compared with the established LAT : Semiquantative results can reliably be achieved within a minimum of time. The new assay can, therefore, improve routine as well as emergency diagnostics. in conciusion, cardiopulmonary bypass surgery leads to an activation of hemostasis probably contributed by cytokines. Soluble fibrin and D-dimers proved to be excelient parameters in monitoring the activation and counter-regulation of coagulation and fibrinolysis.

Universitätskliniken, D-37075 Göttingen, FRG
COMPARISON OF THE BIOCOMPATIBILITY OF HOLLOW FIBER DIALYZER TO FLAT PLATE DIALYZER BY MEANS OF DIFFERENT MARKERS OF BLOOD COAGULATION

\section{H.H. Berger, R.M. Loreth, A. Rieder, D. Skroch* and F.W.} Albert

The influence of the different geometry of hollow fiber dialyzer (Filtral 12) and flat plate dialyzer (Crystal 3400) on blood coagulation activity was investigated on the supposition of a identical membrane composition (polyacrylonitrile). A crossover study comparing 11 patients undergoing regular hemodialysis was performed. Blood for analysis was drawn at five different times (T0T4) during the hemodialysis. Apart from the routine parameters (PT, aPTT. TT, Fibrinogen, Platelets) inhibitors (Antithrombin III, Protein C, Protein S, $\alpha 2$-Antiplasmin) and more recent activation and fibrinolytic markers (TAT, FI/2, D-Dimer, PAI-1, tPA) where evaluated. When applying less sensitive parameters such as D-Dimer and platelets no significant difference was found. Highly significant $(p<0,05)$ changes in TAT and $F 1 / 2$ however suggest a greater influence on blood coagulation in flat plate dialyzer. We conclude that the different geometry and therefore the different effect of shear stress have a major influence on the balance of the hemostatic system.

Klinische Hämostaseologie, Medizinische Klinik III, Klinikum Kaiserslautern, Germany, * Fa. Hospal GmbH, Germany

161

INFLUENCING HAEMOSTASIS DURING CONTINUOUS VENO-VENOUS HAEMOFILTRATION (CVVH) IN ACUTE RENAL FAILURE (ARF)

1. Stefanidis, J. Hägel, and N. Maurin

In cases of ARF, haemostasis is influenced by uraemia, by disseminated intravascular coagulation (DIC) as part of a multi-organ failure and by renal replacement therapy (heparin, contact with foreign surfaces). In order to establish and quantify the influence of CVVH on coagulation, we studied 14 ARF patients treated by CVVH. Patients with thromboembolic complications, DIC or artificial heart valves were excluded from the study. Coagulation tests were performed prior to, at threeday intervals during and after completion of CVVH. Fibrinopeptide A and thrombin-AT III complex were significantly raised before commencement of CVVH, with values of $31 \pm 19 \mathrm{ng} / \mathrm{ml}$ (ref. $<3.0$ ) and $11 \pm 5 \mathrm{ng} / \mathrm{ml}$ (ref. 1.0 4.0) respectively. There was no rise in the two parameters during therapy (fibrinopeptide $A: 21 \pm 18, p<0.05$; thrombinAT III complex: $12 \pm 9, \mathrm{p}=0.18$ ). Platelet retention (Hellem II) was tower at $35 \pm 29 \%$ (ref. 60-100) and B-thrombogtobutin raised to $121 \pm 45 \mathrm{IU} / \mathrm{ml}$ (ref. 10-40). During CVVH there was a fall in platelet retention to $16 \pm 13 \%$ and an increase in the $B$ thromboglobin/creatinine ratio from 0.39 to 0.65 . Apart from frequent filter acclusion there were no thromboembolic complications. A significantly lengthened filter running time ( $p$ $<0.05)$ was observed with increasing activated clotting time (ACT) and an increased heparin dosage $(r=0.3, p<0.05)$ with a rising platelet count. There was, however, no influence of fibrinogen, thrombin time, platelet count or haematocrit on filter running time. A significant plasmatic coagulation activation occurs in ARF. CVVH leads to additional platelet activation, without influencing plasmatic coagulation. Given adequate heparinization, filter running time depends solely on the ACT value.

Medical Clinic 11, University Hospital Aachen, D-52057 Aachen, Germany 
INCREA SE OF INTERLEUKIN-6 (IL-6) AND INTERLEUKIN-8 (IL8) DURING ORTHOTOPIC LIVER TRANSPLANTATION (OLT) G. Himmelreich, M. Jochum, P. Neuhaus, M. Riewald, and H.Riess

There is a strong interaction recognized between the inflammatory response and hemostasis. In OLT recent investigations have indicated that enhanced leukocyte activation takes place with reperfusion of the graft liver. In parallel, an postreperfusional increase of thrombomodulin (TM) indicating alteration of endothelial cells and a DIC-like situation were observed. In order to further investigate the pathopysiology of leukocyte activation and endothelial damage during OLT we investigated the course of IL-6, IL-8, TNF, elastase (EPI) and TM in the course of 46 OLTs.

Results:

1.TNF levels showed a steep and sustained increase with revascularisation of the graft liver.

2. IL-6 and IL-8 exhibited a parallel course during OLT with moderate but highly significant increases during preanhepatic and anhepatic phases followed by an explosive increase in the reperfusion phase reaching maximal values 60 minutes after the onset of reperfusion.

3. Concentrations of EPI increased steadily and highy significantly throughout the whole operation reaching maximal values 60 minutes after the onset of reperfusion.

4. Soluble TM levels increased significantly during the anhepatic phase followed by a more prominent and highly significant increase in the early reperfusion phase.

5. Significant correlations were found between TM and IL-6 in the anhepatic phase as well as between EPI-complexes, IL-6 and IL-8 in the reperfusion phase.

Conclusion: During the anhepatic phase endothelial damage is connected with an increase of $\mathbb{L}-6$, wherease in the reperfusion phase cytokines like TNF, IL-6, and IL-8 in addition to EPI may play an important role in the DIC-like constellation obseved.

Medizinische Klinik und Poliklinik, Universitätsklinikum Rudolf Virchow, Augustenburger Platz 1, 13353 Berlin
RAPId ONSET of Crtokine-Mediated Fibrinolysis and SubseQuent ACtivation of Coagulation in a Model of Acute SePticemia J.U. Wieding, F.H. Otto, K. Vehmeyer, K.F. Kölmel and E. Günther

In an immunotherapeutic trial on disseminated malignant melanoma, 16 patients intravenously received either the Iysate from streptococci (Picibanil) or from serratia marcescens and streptococci (Vaccineurin). Cytokines and hemostatic parameters were monitored in samples drawn at intervals of $1.5 \mathrm{~h}$ beginning with the injection of the bacterial lysate.

Increase in cytokines began immediately after the bacterial lysate injection. Tumor necrosis factor -alpha (TNF) peaked after $1.5 \mathrm{~h}$ following the Vaccineurin injection with 105-fold elevated levels. Interleukin-6 (IL-6) increased to a maximum of ca. 260 -fold elevated values $3 \mathrm{~h}$ after injection, whereas gamma-interferon (IFNg) showed a late, but sudden increase only after Picibanil (but not after Vaccineurin) resulting in a peak during the fever maximum (ca. 5 to $6 \mathrm{~h}$ ). No significant changes could be detected in concentrations of $1 \mathrm{~L}-1,1 \mathrm{~L}-2$ and $\mathrm{LL}-4$.

As a conseqence, hemostasis was subsequently activated. Notably, the onset of fibrinolysis preceded thrombin generation. Fibrin(ogen) degradation products (FtDP) increased up to 3.5 -fold already $3 \mathrm{~h}$ after injection. This early activation of fibrinolysis corresponded with the rapld increase of plasminogen-activator (tPA) and plasmin-antiplasmin complexes (peaks at $60 \mathrm{ug} / \mathrm{l}$ and $4.8 \mathrm{mg} /$, resp.), directly following the TNF-maximum. In contrast, thrombin-antithrombin complexes, prothrombin fragments and fibrinopeptide A peaked with 3.5 to 8 -fold elevated values $1.5 \mathrm{~h}$ later. The strong parallelism of these 3 parameters was notable. The values reached their maxima closely following the IL-6 peak, 1.5 to $3 \mathrm{~h}$ after the TNF peak, and reattained basal levels $18 \mathrm{~h}$ later. Protein $\mathrm{C}$ declined by $15 \%$ within $6 \mathrm{~h}$ whereas antithrombin III remained unchanged; the platelet number fell by $12 \%$. Fibrinogen was quite stable but began to increase $6 \mathrm{~h}$ after injection, paralleled by $a_{1}$-antitrypsin and C-reactive protein (increases by 30 to $60 \%$ within 1 day). Altogether, changes in cytokines and hemostatic parameters occurred earlier and were comparable but more pronounced (in extent and duration) after injection of endotoxin-containing lysates compared to gram-positive bacterial lysates; only IFNg showed a different course. The changes of hemostatic factors were compensated and normalized within a few hours.

Conclusively, this phase II study may also serve as a model for a short, acute septicemia. The results provide insights into the dynamics of cytokine-mediated processes such as acute phase protein reaction or activation of hemostasis as well as the relevance of markers for thrombinemia and fibrinemia.

Universitätskliniken, D- 37075 Göttingen, FRG

\section{Hirulin}

165

ANTICOAGULANT AND ANTITHROMBOTIC ACTION OF NOVEL INHIBITORS OF THROMBIN

J. Stürzebecher*, P. Wikström, H. Vieweg, J. Meier and $\mathrm{K}$. Stocker

A series of novel potent inhibitors of thrombin containing 3-amidinophenylalanine as the key building block was studied. The compounds inhibit thrombin competitively with $k_{f}$-values near 1 nmol/1. In vitro TT, aPTT and PT, respectively, are doubled by these substances at concentrations of $0.05,0.5$ and 1 pmol/1.

In rats, the blood levels decreased rapidly after i.v. application, however, significant blood levels were observed after oral administration. High levels were found after (intra)duodenal administration. In vivo the anticoagulant effect (aPTT) was slightly stronger, indicating a possible inhibitor accumulation in blood corpuscles and/or the endothelium of the vessels.

In rats, the inhibitors prevented the formation of experimental venous thrombi according to WESSLER.

Present address: *Institut für Pharmakologie \& Toxikologie, Medizinische Hochschule Exfurt, D-99012 Erfurt; Pentapharm Ltd., CH-4002 Basel
166

PHARMACOKINETICS OF NOVEL INHIBITORS OF THROMBIN J. Stürzebecher*, P. Wikström, H. vieweg, J. Meier, D. Prasa*, C. Adler and K. Stocker

Novel potent inhibitors of thrombin ( $\mathrm{K}_{j}$-values near 1 nmol/1) containing 3-amidinophenytalanine as the key building block were studied. The pharmacokinetics were evaluated in rats using an HPLC detection method. The blood levels decreased rapidly after i.v. application. However, significant blood levels were observed after oral administration. High levels were found after rectal and (intra)duodenal administration. Significant enteral absorption was never observed with benzamidine-derived thrombin inhibitors so far.

while the inhibitors were rapidly cleared after $i . v$. administration ( $t \frac{1}{2}$ near $10 \mathrm{~min}$ ), $t \frac{1}{2}$ after duodenal uptake was significantly longer indicating a possible accumulation of the inhibitors. Therefore, the fate of some compounds in the organism was studied.

Present address: *Institut für Pharmakologie \& Toxikologie, Medizinische Hochschule Erfurt, D-99012 Erfurt; Pentapharm Ltd., $\mathrm{CH}-4002$ Basel 
GLOMERULAR FILTRATION AND TUBULAR REABSORPTION ARE INVOLVED IN RENAL ELIMINATION OF HBW 023 M. Hropot, F. Pietruck*, H. Grötsch, E. Klaus, P. Hainz, and H.-J. Friesen $\$$

Pharmacokinetic studies of the recombinant hirudin, HBW 023 , a potent and selective inhibitor of thrombin, have displayed that the renal clearance of HBW 023 lies below the glomerular filtration rate, indicating tubular reabsorption of this anticoagulant. Moreover, the urinary excretion of HBW 023 amounts to about $50 \%$ of the administered dose in man and only about $5 \%$ in rat. The aim of the present study was to examine the urinary excretion of HBW 023 in dose-response experiments in rats. HBW 023 was injected intravenously in doses of $0.5,1.0,2.5,5.0,50$, and $100 \mathrm{mg} / \mathrm{kg}$ b.wt. to conscious rats. 24-hour urine samples were collected and HBW 023 concentration was determined. When HBW 023 was measured by chromogenic thrombin inhibition assay (CTA), its urinary excretion increased dose-dependently from $2.7 \%$ to about $45 \%$ of the administered dose. In contrast, the ELISA method revealed only $1.6 \%$ of the parent compound in the urine. A simultaneous intravenous administration of HBW 023 and aprotinin caused a moderate increase in HBW 023 excretion to about $6 \%$ as compared to $3 \%$ in control. In a second series of experiments the shot-injection method was applied in rats to evaluate the short-term excretion rate of ${ }^{125}$ HBW $023(0.1$ and $1.0 \mathrm{mg} / \mathrm{kg}) .{ }^{14} \mathrm{C}$ inulin was used as a filtration marker. After the injection of the lower dose the urinary excretion of the labelled compound amounts to about $5 \%$ which corresponded to $8 \%$ of the filtered load. A successive injection of the higher dose resulted in excretion of $39 \%$ of the administered dose. When the higher dose was administered first the excretion rate and filtration fraction of the lower dose of $125 \mathrm{I} \mathrm{HBW} 023$ injected successively were doubled. In conclusion, glomerular excretion as well as tubular reabsorption are involved in the renal excretion of HBW 023. Its reabsorption seems to be mediated by endocytosis being saturable and only weakly inhibited by aprotinin. Very small amounts of the parent compound are excreted in the urine indicating its intralysosomal degradation.

Hoechst AG, 65926 Frankfurt am Main, Germany

*Max-Planck-Institut für Biophysik, 60596 Frankfurt am Main, Germany

§Behringwerke AG, 35001 Marburg, Germany

Potential Hirudin Antidote: Investigation of an Activated Prothrombin Complex Concentrate in Animal Models

Diehl K.-H. . Römisch J. . Hein B., Jessel A.,

Ronneberger H. . Paques E.-P.

Behringwerke AG, 35001 Marburg / Germany

In experimental models, specific thrombin inhibition by $r$-hirudin ( $H B W$ 023) has been demonstrated to be effective in preventing thrombosis. Even though bleeding complications have not been observed using therapeutically effective doses in animal studies, inadvertent overdosing may result in major. impairment of coagulation. As a potential antidote, an activated prothrombin complex concentrate (APC) was tested on its ability to normalize blood coagulation. APC given as bolus injection 5 minutes after termination of one hour $r$-hirudin infusions of concentrations between 0.1 and $3.0 \mathrm{mg} / \mathrm{kg}$, neutralized the $r$-hirudin induced prolongation of whole blood clotting time in rabbits completely within 5 minutes. Histological examination revealed no clot formation in the blood vessels or capillaries of the heart, kidneys or lungs.

Furthermore, bleeding time prolongation induced by bolus application of 3.0 or $30.0 \mathrm{mg} / \mathrm{kg}$ of $\mathrm{r}$-hirudin was significantly antagonized by APC within 5 minutes. Administration of APC may be an effective way to reverse the effects of $r$-hirudin on coagulation in case of inadvertent overdosing of r-hirudin.
IN VITRO DEGRADATION OF HBW 023 IN RAT URINE SAMPLES AND IN KIDNEY HOMOGENATES FROM RAT AND DOG

H. Grötsch, M. Hropot, H.-J. Friesen*, E. Dichtl, O. Gnau*, R. Ben Youssef, and U. Schwarzer

Recombinant hirudin HBW 023 is a highly potent and selective inhibitor of thrombin which is well-tolerated and can be used parenterally. Pharmacokinetic studies have revealed the elimination half-life of about 1 hour in man and in animals. However, there exist pronounced differences in urinary excretion of HBW 023 in different species. In man about $50 \%$ of the administered dose are excreted in urine, whereas in rat there are only about $5 \%$ when HBW 023 is measured by chromogenic thrombin inhibition assay (CTA). Little is known about renal handling and metabolism of HBW 023. Aim of the present experiments was to examine HBW 023 degradation caused by renal enzymes excreted in the urine and by kidney homogenates. For this purpose rat urine samples were kept at room temperature up to 24 hours after addition of sodium azide as preservative at the original $\mathrm{pH}$ value. Analysis of $\mathrm{HBW} 023$ was performed by the CTA method and by two monoclonal sandwich ELISAs [Antibody A65 with an epitope around position 20 as conjugate antibody in combination with antibody A60 with an epitope around position 60 (Pro) or antibody $A 810$ with an epitope at position 63-65 (Tyr-Leu-Gin-OH) as solid phase antibody]. After the incubation of HBW 023 at the $\mathrm{pH}$ values between 6.15 and 9.04 about $83 \pm 8 \%$ of thrombin inhibitory activity was found with CTA method as compared to only $45 \pm 25 \%$ measured by the ELISA methods. From rat and dog kidneys total homogenates and cytosolic fractions were used for degradation studies at $37^{\circ} \mathrm{C}$ during 30 minutes. Decomposition of HBW 023 was about $50 \%$ higher in rat kidney homogenate and cytosolic fractions as compared to those of dogs.

In conclusion, the comparison of CTA method with two different ELISA methods suggest that the CTA method covers the total thrombin inhibitory activity of HBW 023 , whereas the ELISA methods more specifically determine the undegraded parent compound.

Hoechst AG, 65926 Frankfurt am Main, Germany

*Behringwerke AG, 35001 Marburg, Germany

\section{RECOMBINANT HIRUDIN (CGP 39393) AS ANTICOAGULANT IN}

170 EXPERIMENTAL HEMODIALYSIS AND HEMOFITRATION

Lïjers S. Schutz ERuschitzixa F, Heydenbiuth R. Gronau C. Zutchner C. Ekman $\mathrm{S}^{0}$, Kobsterng H", Schrader I

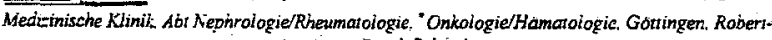
Koch-Sr. 40, 37075 Göttingen, ${ }^{\circ}$ Ciba-Geig: Bosel, Schwieiz

Hirudin is the most effective and selective direct (independent of plasma-cofactors) inhibitor of Thrombin. Therefore Hirudin could be of advantage for anticoagulation during hemodialysis in patients with acute renal failure and coagulation-disorders. CGP 39393 (CIBA-GEIGY, CH-Basle) is available for the last few years. Methods: In 21 in vitro-experiments we examined the elimination of CGP 39393 from a circuit by means of dialysis or filtration. In 11 additional in-vivo-experiments functional nephrectomized pigs underwent hemofiltration with high-flux-membranes (HF 80R, Fresenius), which had shown permeability for CGP 39393 in our in vitro experiments. During the 6-hour tests plasma-and filtrate-levels of Hirudin, different blood-coagulation-parameters, blood-picture, as well as clinical effects of the Hirudin-application on the circulatory system and bleeding complications were determined.

Results: It was possible to eliminate CGP 39393 completely with HF 80 in vitro out of the circuit within $1-2$ hours. In vivo hemofiltration with a dosage of $1 \mathrm{mg} / \mathrm{kg}$ body weight i.v. (given as a bolus) and a continous i.v. infusion of $0,5 \mathrm{mg} / \mathrm{kg}$ BW without complications or significant effects on the circulatory system was possible. With Hirudin-plasma-levels below $1000 \mathrm{ng} / \mathrm{ml}$ the extracorporal system started to dewelop thrombi. CGP 39393 was not eliminated completely by the end of the experiment. The blood-coagulation times did not compare to the baseline-times. A control of the therapy was especially possible with aPTT. Summary: CGP 39393 proved to be good controlable and effective substance for effective anticoagulation in hemofiltration in functional nephrectomized pigs. Unwanted side effects or bleeding complications did not occur despite an increased bleeding risk of the recently operated animals. 
171

Predictable Additive Anticoagulant Effects of

Römisch J.. Diehl K.-H. , Jessel A.

Behringwerke AG, 35001 Marburg, Germany

In prophylaxis or therapy of thrombosis, patients initially treated with heparin may subsequently receive recombinant ( $r$-) hirudin, a novel thrombin-inhibitor currently in clinical investigation. Prevention of thrombotic events by heparins or $\mathrm{r}$-hirudin is effected by different inhibitory mechanisms. Therefore, we investigated potential additive effects of heparin / r-hirudin ccmbinations in vitro. aPTT turned out to be the most suitable parameter, whereas PT or Thrombin Time are limited for this purpose. Starting with the separate anticoagulants, standard human plasma (SHP) was spiked either with low molecular weight heparin (LMWH. Fraxiparin $^{\mathrm{R}} 0.3$; SANOFI Gmb/Germany; $0-20 \mathrm{\mu g} / \mathrm{ml}$ ), unfractionated heparin (UFH, Liquenin ${ }^{R}$; HOFFMANN-LA ROCHE AG / Germany; $0-0.5 \mathrm{IU} / \mathrm{ml}$ ) or $\mathrm{r}$-hirudin (HBW 023: BEHRTNGWERKE AG / HOECHST AG / Germany; $0-2 \mu \mathrm{g} / \mathrm{ml}$ ). aPTT was determined using a coagulometer according to Schnittger and Gross. Doubling of aPTT was achieved employing concentrations of approximately $7.5 \mathrm{\mu g} / \mathrm{ml}$ of LMWH, $0.25 \mathrm{IU} / \mathrm{ml}$ of UEH or $0.75 \mathrm{\mu g} / \mathrm{ml}$ of r-hirudin. Stocking up LMWH- or UFH-spiked SHP with increasing concentrations or r-hirudin, the anticoagulant effects were more pronounced as demonstrated by the prolongation of aPTT. The anticoagulant effects are additive and predictable in concentration ranges of both inhibitor types usually found in patient plasma, with no evidence of potentiating interactions. Projecting these in vitro results toward the in vivo situation, there is low risk of inadvertent over-proportional anticoagulant effects, which might enhance bleeding tendency. Animal studies have been started to confirm these in vitro results. r-Hirudin (HBW 023) and Heparins in Vitro

\section{3}

COMPARATIVE SERINE PROTEASE INHIBITORY EFFECTS OF RECOMBINANT TISSUE FACTOR AND RECOMBINANT HIRUDIN. D. Callas, D. Hoppensteadt, W. Jeske, J.M. Walenga and Jawed Fareed. Loyola University Chicago, Stritch School of Medicine, Maywood, IL. 60153.

Selective inhibition of serine proteases by their physiologic inhibitors (serpins) can lead to selective manipulation of clotting, fibrinolytic and platelet functions. Tissue Factor Pathway Inhibitor (TFPI) is a serpin with a broader inhibitory spectrum which also includes non-serine proteases. While TFPI is known to act by inhibiting TFN/la and factor $\mathrm{Xa}_{\mathrm{a}}$, its interactions with other serine proteases remains unknown. Since recombinant hirudin (Knoll AG, Ludwigshafen, Germany) represents the most specific inhibitor of thrombin, which is considered by many to have a pivotal role in the regulation of hemostatic processes, we have compared the antiprotease and protease generation inhibitory actions of this agent with recombinant TFPI (Monsanto, Chesterfield, $\mathrm{MO}$ ). The direct serine protease inhibitory effects of these two agents was measured in defined systems where the activity of purified serine proteases was detected with specific substrates. In some of the protease generation systems, the inhibition of the generation of thrombin, factor Xa and kalikerein from fibrinogen deficient plasma was detected, after activation with dextran or actin (intrinsically), or recombinant tissue factor (extrinsically). The proteases generated were measured using specific synthetic substrates on an automated centrifugal analyzer (ACL 300 plus, Instrumentation Laboratory, Lexington, MA). TFPI produced a strong inhibition of factor $X_{a}\left({ }_{6} C_{60} \leq 3.0\right.$ $\mathrm{nM}$ ). No other serine proteases were inhibited at concentrations of up to 100 $\mathrm{nM}$. On the other hand, hirudin did not inhibit any of these serine proteases, exception for thrombin $\left(1 \mathrm{C}_{60} \leq 1.0 \mathrm{nM}\right)$. The two recombinant proteins produced varying degrees of inhibition of thrombin and factor $X a$ generation in various systems. In the extrinsic protease generation assay, TFPI produced considerably stronger inhibition $\left({ } C_{60}\right.$ for thrombin $=10 \mathrm{nM}$, factor $\mathrm{Xa}=50$ $\mathrm{nM}$ ). In contrast, r-hirudin was a relatively poor inhibitor of both the thrombin and factor Xa generation in the plasma systems $\left({ } C_{60}\right.$ for thrombin $=40 \mathrm{nM}$, factor $X a \geq 500 \mathrm{nM}$. These studies suggest that TFPI is a multi-targeting protease inhibitor and plays an important role in the regulation of hemostatic processes. In contrast, $r$-hirudin produced a direct and specific antithrombin action without significant non-thrombin interactions. These findings suggest that agents exhibiting a direct potent inhibition of thrombin may not necessarily be equally effective in the inhibition of thrombin generation.

\section{PHARMACOLOGICAL CHARACTERIZATION OF THE NEW 4- AMIDINOPHENYLALANIN (ADF) THROMBIN-INHIBITOR (CRC 220)}

G.Dickneite ${ }^{1}$, D.Seiffge ${ }^{2}$, K.H.Diehl ${ }^{1}$, M.Reers ${ }^{1}$, J.Czech ${ }^{1}$, E.Weinmann ${ }^{1}$, D.Hoffmann 1 , W.Stüber ${ }^{1}$

Thromboembolic disorders represent the major cause of morbidity and mortality in industrialized countries. Intravascular activation of clotting leads to myocardial infarction, stroke, pulmonary embolism, deep vein thrombosis and disseminated intravascular coagulation (DIC). We designed a series of new thrombin inhibitors, the most promising being 4-methoxy-2,3,6-trimethylphenylsulphonyl-L-Asp-D-Adf-piperidide (CRC 220) with a Ki value of $2 \mathrm{nM}$ and a high specificity for thrombin. CRC 220 lead to a dose dependent prolongation of aPTT, PT and TT as determined in rats, rabbits, dogs, sheeps and monkeys. We evaluated the efficacy of CRC 220 to prevent thrombus formation. In a rabbit model of tissue factor induced coagulation activation, infusion of $0,5 \mathrm{mg} / \mathrm{kgxh}$ CRC 220 (3 hours) leads to a significant prevention of fibrinogen decrease and the increase in fibrin monomers. In a rat model of lethal LPS induced DIC, CRC 220 dose dependently prevented the mortality rate, an ED50 of $0.17 \mathrm{mg} / \mathrm{kgxh}$ (4h infusion) was obtained. Thrombin induced platelet aggregation in rat lungs could be prevented by the i.v. bolus injection of CRC $220.0 .3 \mathrm{mg} / \mathrm{kg}$ leads to a more than $80 \%$ reduction of ${ }^{51} \mathrm{Cr}$ labelled platelets deposition in the lung, inhibition was still observed 2 hours after CRC 220 administration, at this time point the inhibitor had already been cleared from plasma. Arterial thrombosis was, induced in rabbits by stenosis of the $A$. carotis. The i.v. bolus aministration of CRC 220 dose dependentely prevented thrombus formation, an ED50 of 0.03 $\mathrm{mg} / \mathrm{kg}$ was calculated. It could thus be concluded, that this thrombin inhibitor should have beneficial effects in the treatment of thrombotic disorders.

1 Research Laboratories of Behringwerke AG, D-35001 Marburg, FRG;

2 Hoechst AG, Werk Kalle-Albert, D-65174 Wiesbaden, FRG
INFLUENCE OF THROMBIN INHIBITORS ON PROTHROMBIN ACTIVATION INDUCED BY SNAKE VENOMS

Alike the prothrombinase complex, several proteases contained in snake venoms activate the conversion of prothrombin to thrombin via intermediates. For example, the venoms of Echis carinatus, Bothrops neuwiedi, Notechis scutatus, and of Dipholidus typus contain enzymes which, by limited proteolysis, liberate thrombin. While every single step of the activation mechanism induced by $\mathrm{E}$. carinatus venom (Ecarin) is known in detail, the mechanisms of prothrombin activation induced by other venoms have been studied less. Using cleavage of a chromogenic substrate ( $N$-p-tosyl-Gly-Pro-Arg-p-nitroanilide), the serine proteinase activity generated by prothrombin activation could be detected in the four venoms and the course of activation was followed. First, equieffective doses of the venoms were ascertained with respect to substrate cleavage. The use of different types of thrombin inhibitors such as heparin, hirudin, and synthetic inhibitors (e. g., $\alpha$-NAPAP) was aimed at getting information on the activation mechanisms of the various venom proteases studied. To achieve a $50 \%$ enzyme inhibition, for all venoms almost the same concentration of the low molecular weight inhibitor $\alpha$-NAPAP acting on the active site is required. In contrast to this, the activities of Bothrops neuwiedi and Dipholidus typus venoms are inhibited only at considerably higher heparin concentrations, compared with Notechis scutatus venom. Heparin is known to catalyze the formation of the inactive thrombin/antithrombin complex only and to not react with intermediates. Hirudin reacts with thrombin and also with meizothrombin and meizothrombin-(des F1). It inhibits the rate of cleavage released by $E$. carinatus, Bothrops neuwiedi, and Dispholidus venom within the same dose range. The action of Notechis scutatus venom, however, is inhibited already at considerably lower hirudin concentrations. From these preliminary results it can be concluded that the effect of Notechis scutatus venom is obviously markedly different from the other three venoms.

Max-Planck-Gesellschaft e. V., Research Unit for Pharmacological Hemostaseology at the University of Jena, Drackendorfer Str. 1, D-07747 Jena, FRG
R. Petrovan, P. Walsmann, and G. Nowak 
HEPARIN INDUCED THROMBOCYTOPENIC POTENTIAL OF UNFRACTIONATED HEPARIN AND LOW MOLECULAR WEIGHT HEPARINS AS MEASURED BY A FLOW CYTOMETRIC METHOD. J.M. Walenga, M.J. Koza, I.V. Shankey," D. Hoppensteadt" J. Fareed V. Lonchyna," R. Pifarre," E.W. Bermes, Jr." Loyola University Medical Center, Maywood, IL.

Heparin induced thrombocytopenia (HIT) has generally been considered to be an immune-mediated response to heparin. Flow cytometric studies have shown the platelet Fc-receptor expression could be affected in the presence of heparin and antibodies that bind to other receptors such as GP $\mathrm{lb}$ or $\mathrm{Ilb} / \mathrm{lll}$. However, no one has used flow cytometry as a diagnostic tool for detecting this syndrome. Ten normal donors were carefully drawn using a double-syringe technique without tourniquet using either unfractionated heparin, a low molecular weight heparin (LMWH) Enoxaparin ${ }^{n}$, or a semi-synthetic LMWH at $10 \mathrm{ug} / \mathrm{ml}$ final concentration. Aliquots were immediately fixed in $1 \%$ paraformaldehyde from whole blood and then added to either normal heatinactivated serum, strong HIT positive heat-inactivated serum, or saline and incubated at $37^{\circ} \mathrm{C}$ for 15 minutes. Native whole blood was also added to arachidonic acid to serve as a positive control. After 15 minutes aliquots were fixed and incubated at $4^{\circ} \mathrm{C}$ for 30 minutes. The samples were centrifuged, resuspended in calcium free tyrodes buffer and stained with CD61 (GPIIb/IIIa)FITC (Becton-Dickinson) and CD62 (GMP-140)-PE (Becton-Dickinson] monoclonal antibodies. Samples were incubated for 30 minutes in the dark at room temperature and then analyzed on either a FACScan (Becton-Dickinson) or Elite XL MCL (Coutter). Four of ten normal donors demonstrated positive results with HIT positive serum and no positive results were seen in either the saline or normal serum control. No positive reaction was seen in either of the LMWHs with HIT positive sera. This data demonstrates a lack of crossreactivity to the HIT associated antibody with the LMWHs tested. Furthermore, this study demonstrates the possible use of the flow cytometer for the diagnosis of HIT. Advantages over the routine platelet aggregation method include small sample size and use of patient's own platelets/serum which may avoid the false positive/negative results obtained by the platelet aggregation method.

\section{STUDIES ON THE PROFIBRINOLYTIC EFFECT OF THE POLYSULFONATE GL 522}

U. Kurtze ${ }^{1}$, B. Åstedt ${ }^{2}$, J. Fareed ${ }^{3}$, and H.-P. Klöcking 1

GL 522 (Genelabs, Redwood City, Cal) is a newly developed polysulfonate with antithrombotic properties. We have investigated the effects of this compound on fibrinolytic components released from the vessel wall. For this purpose the isolated perfused pig ear model was used (Klöcking et al. Sem. Thrombos. Hemostas. 17:379, 1991). The perfusion scheme was as follows: four fractions with Tyrode's solution $\left(2 \mathrm{ml} / \mathrm{min}, 37^{\circ} \mathrm{C}, \mathrm{pH} 7.4\right.$ ), then two fractions (five and six) with Tyrode's solution containing GL 522, and, finally, six fractions with Tyrode's solution alone. The fractions were collected at 2-minute intervals. Three concentrations were tested: 10,50 ,

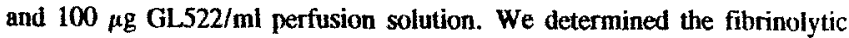
activity on plasminogen-rich plates, the t-PA and the PAI-1 antigen concentrations. At the same time we recorded pressure and volume per fraction. On perfusion with $100 \mu \mathrm{g} / \mathrm{ml}$ perfusion solution the t-PA and PAI antigen concentrations were significantly enhanced in fraction nos. 5-8, while an increase in pressure and a reduction in volume was found in fraction nos. 5 and 6 . The increase in fibrinolytic activity amounted to $100 \%$. Perfusion with $50 \mu \mathrm{g}$ GL 522 showed qualitatively the same results whereas the PAI-1 antigen concentration remained below the detection limit. These results prove the profibrinoytic activity of GL 522 .

Institute of Pharmacology and Toxicology, Medical School Erfurt, PB 595, D 9912 Erfurt, Germany ${ }^{1}$

Research Laboratory of the Department of Gynaecology and Obstetrics, University of Lund, University Hospital, S-22185 Lund, Sweden ${ }^{2}$

Departments of Pharmacology and Pathology, Loyola University Medical Center, Maywood, IL 60153, USA ${ }^{3}$

178

\section{ACUTE t-PA RELEASE BY DIFFERENT SUPERSULPHATED HEPARINS \\ H.-P. Klöcking 1 and A. Butti ${ }^{2}$}

CUIAR WHEIGHT HEPARIN ON BLOD COAGULATION AND PLATELET FUNCTION

J.F. Schenk, P. Radziwon*, P. Boczkowska-Radziwon, A. Butti**, E.Glusa ***. H. K. Breddin *

In a phase I clinical trial on the newly developed supersulfated low molecular weight heparin (SSH Iketon Pharmaceutici Milan) six healthy volunteers received $50 \mathrm{mg}$ SSH subcutaneously. Coagulation and platelet function parameters were studied 30 minutes and $1,2,4,8$ and 12 hours after the s.c. injection.

Parameters studied were aPTT, Thrombin time, Heptest, anti $\mathrm{Xa}$ activity, anti lla activity, platelet adhesion, platelet count, platelet induced thrombin generation time, and bleeding time.

PTT was significantly prolonged $30 \mathrm{~min}$ to 4 hours after the sc injection of SSH and Heptest was significantly prolonged up to the eighth hour while the preinjection values were reached after 12 hours. The PITT TC values were moderately but significantly prolonged after $480 \mathrm{~min}$. Platetet adhesion was not affected, Thrombin time was only slightly prolonged 4 hours after the injection. No anti lla-activity could be measured, during the study. Bleeding time was slightly but significantly prolonged two hours after the injection, but remained in the normal range.

Further investigation will have to verify if SSH is an effective antithrombotic agent to prevent thromboses in high risk patients.

- Division of Angiology, Department of Internal Medicine, J.W. Goethe-University Frankfurt am Main

* * Iketon Pharmaceutics Milan

*** Institute of Pharmakology Medical Academy Erfurt. Germany

Deutscher Titel: Wirkung eines neuen supersulfatierten niedermolekularen Heparins auf Blutgerinnung und Plättchenfunktion
Different supersulphated heparins (SSH) were isolated after controlled depolymerisation and contemporary sulfation of pig mucosal heparin (UFH) (Diosynth) and tested for acute t-PA release in the isolated perfused pig ear at concentrations of 50 and $100 \mu \mathrm{g} \mathrm{SSH/ml} \mathrm{perfusion} \mathrm{solution.}$

Table 1 Supersulphated heparins (SSH)

\begin{tabular}{|c|c|c|c|c|}
\hline \multirow[t]{2}{*}{ Substance } & \multirow[t]{2}{*}{ m.w. } & \multirow[t]{2}{*}{$\mathrm{SO}_{3 / \mathrm{COO}^{-}}$} & \multicolumn{2}{|c|}{$\begin{array}{l}\text { increase of } t-P A \\
\text { activity }(\%)\end{array}$} \\
\hline & & & $50^{x}$ & $100^{x}$ \\
\hline UFH & $\begin{array}{l}15000- \\
20000\end{array}$ & 2.2 & 54 & 65 \\
\hline $\mathbf{S S H}_{11}$ & 12200 & 3.5 & 74 & 150 \\
\hline $\mathrm{SSH}_{12}$ & $\begin{array}{r}10100 \\
5400\end{array}$ & $\begin{array}{l}3.9 \\
4.4\end{array}$ & $\begin{array}{l}50 \\
38\end{array}$ & $\begin{array}{l}36 \\
31\end{array}$ \\
\hline $\begin{array}{l}\text { SSH13 } \\
\text { SSH }_{14}\end{array}$ & 3500 & 4.1 & 90 & 75 \\
\hline control /wi & stance) & & 7 & \\
\hline
\end{tabular}

( ${ }_{\mu \mathrm{g} / \mathrm{ml} \text { perfusion solution) }}$

According to these results, increased sulfation of SSH substances is partly connected with increased efficacy. This is true for the high molecular weight $\mathrm{SSH}_{11}$ and the low molecular weight $\mathrm{SSH}_{14}$.

Institute of Pharmacology and Toxicology, Medical School Erfurt, FRG ${ }^{1}$ and Iketon Farmaceutici s.r.1., Segrate, Italy ${ }^{2}$ 
MYCOBACTERIAL SORPTION TO THE BLADDER WALL: AN APPROACH CONSIDERING THE PHYSICOCHEMICAL SRORACE AND EFFECTS OF EXOGENOUS POLYELECTROLYTES. D.H.J. Schamhart, E.C. de Boer and K.H. Kurth

The role of glycoaminoglycans (GAGs) in the attachment of bacteria to the (epithelial) layer, interest. Intravesical is instillation of BCG (bacillus calmette-Guérin) is used for treatment (bacilicicial bladder cancer. The role of BCG attachment to the lumenal surface of the bladder tigated in a guinea pig model. In this model the effects on modulating BCG attachment by exogeneous GAGS can be monitored by BCG-induced immunological reactions. Computer modeling based on the theory of lyophobic colloid stability, suggests that in the uninjured bladder, BCG attachment to the bladder wall is very low, due to a high, electrostatic repulsion barrier. A profound effect of ed, depending severely however on the GAG concentrátion and surface properties. A significant binding of pentosan polysulfate (PPS), a highly sulfated semisynthetic GAG to both the guinea pig bladder ( $76 \pm I \mu \mathrm{I})$ and BCG (3.4 $\pm 0.3 \mu \mathrm{g} / \mathrm{mg} \mathrm{gry}$ weight) was observed. Binding of PPS to both the enhancement of BCG attachment by "bridging" (low (high PPS concentrations). The effects of intrayesical PPS (10 and $\left.0.1^{\circ} \mathrm{mg} / \mathrm{ml}\right)$ on BCG-induced imane reactions were studied in the quinea pig. In contrast to $10 \mathrm{mg} / \mathrm{ml}$ PPS, preinstillation with $0.1 \mathrm{mg} / \mathrm{ml}$ PPS resulted in a significant ( $0<0.05$ ) increase of the various immunological parameters. concentrations $(0.1 \mathrm{mg} / \mathrm{ml})$ enhance $\mathrm{BCG}-$ induced immune reactions in the guinea pig probably as a result of an increased BCG sorption to the bladder wall. It can be speculated that PPs may increase the efticacy. of BCG provided conditions of bridonly one of the surfaces (bladder wall or $B C G$ ).

Dept Urology, Univ, Amsterdam, Meibergdreef 9, Supported by bene Arzneimittel, Germany.

\section{HEPARIN-BINDING TO LEUKOCYTES DEPENDS ON ITS DEGREE OF SULFATION AND THE LIPOPHILICITY} J. Harenberg. R. Malsch. L. Piazolo.M. Schäfer, and D.L. Heene

The anticoagulant actions of heparins are well defined in contrast to the non-anticoagulant properties, which include the antithrombotic, antiinflammatory, antiatherosclerotic or antimetastatic activities. Heparins may influence these diseases through binding to leukocytes. Recently we described the specific labeling of low molcular mass heparin by endpoint attachment of tyramine and fluorescein-5-isothiocyanate (LMMH-tyr-Fitc, Anal Biochem, in press). Binding of LMMH-tyr-Fitc to leukocytes is analyzed by means of flow cytometry. We now report on the influence of the sulfation degree and of the lipophilicity on binding of heparin to leukocytes. Heparin and LMMH displace LMMH-tyr-Fitc to $50 \%$ at equigravimetric doses. Heparin derived dodeca-, deca-, octa-, hexa-, tetra- and disaccharides were less effective decreasing with the molecular weight. The equigravimetric doses increased more than expected due to the decreasing degree of sulfation per oligosaccharide. The pentasaccharide was not the most effective compound indicating an antithrombin III independent heparin binding.

Butyryl was bound to heparin to obtain a lipophilic derivative. The anti-factor $X_{a}$ and anti-factor lla activities were about 120 iU/mg compared with $160 \mathrm{lU} / \mathrm{mg}$ of the parent compound. The ability of butyryl-heparin was 50-fold higher to displace LMMH-tyr-Fitc from leukocytes compared with heparin.

The results demonstrate that binding of heparin depends strongly on the sulfation degree of the oligosaccharides and also on the lipophilicity. Interactions may occur via the L-selectins, which are responsible for binding of oligosaccharides. Lipophilic modification may specifically increase the receptor-affinity of oligosaccharides.

Supported by Deutsche Forschungsgemeinschaft (Ha 1164/3-2) 1. Medizinische Klinik, Fakultät Klinische Medizin Mannheim, Theodor Kutzer Ufer, 68167 Mannheim

\section{1}

Comparison of The Antitcoagulant Effects of LMM-

Heparin-Tyramine, LMM-Heparin-Tyramine-Fitc and Low Molecular Mass Heparin

R. Malsch, L. Piazolo, C. Giese, M. Schäfer and J. Harenberg

The comparison of the pharmacokinetic and pharmacodynamic profile requires labeled heparin and low molecular mass heparin (LMMH). We recently reported on the synthesis of low molecular mass heparin-tyramine (LMMH-Tyr) and low molecular mass heparin-tyramine-fitc (LMMH-TyrFitc). (Anal Biochem, in press) Here we report on the anticoagulant properties of these "endpoint-attached" labeled low molecular heparins. LMMH-Tyr and LMMH-Tyr-Fitc showed normal plasmin activity in whole blood (from 90 to $132 \%$ ) which were comparable to LMMH. Thrombin generation inhibition of modified LMMH in whole blood was as effective as with LMMH. The modified LMMH did not show inactivation over a period of 120 minutes as measured by anti-Xa activity in a whole blood assay. These results demonstrate that the anticoagulant properties of LMMH-Tyr and LMMH-Tyr-Fitc remained unaffected during the synthesis.

Heparin, LMMH, LMMH-Tyr and LMMH-Tyr-Fite (100 aXa U/kg) were injected as a bolus to 8 Sprague-Dawly rats and blood samples were collected at $0,10,30,60,120,240$ and 360 minutes. The statistical analysis was performed using the Mann Whitney U-test. LMMH-Tyr and LMMH-Tyr-Fitc showed a biphasic elimination profile like LMMH. The B-half life of the anti $\mathrm{Xa}$ and anti IIa activity of "endpoint-attached heparins" were significantly prolonged: LMMH-Tyr (122 min), LMMHTyr-Fitc ( $125 \mathrm{~min}$ ) compared to LMMH (69 $\mathrm{min}$ )

LMMH-tyramine and LMMH-tyramine-fitc can be used for the comparison of the pharmacokinetic and pharmacodynamic profile of LMMH and heparin.

Supported by Deutsche Forschungsgemeinschaft (DFG), grant Ha 1164/31 and Ha 1164/3-2

1. Medizinische Klinik, Fakultät für kdinische Medizin Mannheim der Universität Heidelberg, Theodor Kutzer Ufer, 68167 Mannheim

182

PITT SENSITIVITY TO HEPARIN AND HEPARIN-LIKE COMPOUNDS.

P.Radziwon*, B.Boczkowska-Radziwon,J.Schenk, H.K.Breddin.

Platelet-induced thrombin generation time (PITT) is a newly developed global coagulation assay (Haemostasis 1992; 22: 309-321). In this test small amounts of PRP obtained from partially anticoagulated blood (hirudin $0.7 \mu \mathrm{g} / \mathrm{ml}$ ) is rotated in a disc shaped cuvette within the light beam of a photometer. The changes in optical density are recorded. During the rotation platelets are activated and release reaction occurs. Thrombin is formed at the platelet surface and at the cuvette walls and triggers coagulation. The aim of our study was to compare the routinely used coagulation tests (aPTT, Heptest) with PITT in vitro and ex vivo in the detection of hepearin and heparin-like molecules. aPTT and Heptest were performed partially by an automated analyser (ACL, Italy), partialy by an KC 10 analyser (Amelung, Germany). The effect of the following glycosaminoglycanes have been investigated: unfractionated heparins (Calciparin, Sanofi; Liquemin, Hoffmann-La Roche), low mulecular weight heparins (Fraxiparin, Sanofi; Fragmin, Kabi), ultra low molecular mass heparin (Oligo-H OP650, Opocrin), dermatansulfate (DS OP435, Opocrin), and low molecular mass dermatansulfate (LMW-DS 3L, Opocrin). In principle the PITT-system proved to be as sensitive as APTT assay in the detection of heparins and other glycosaminoglycans. Heptest has a minimal advantage over aPTT and PITT as far as sensitivity is concerned. The minimal detectable range for calciparin, fraxiparin and fragmin were for PITT $0.1 \mu \mathrm{g} / \mathrm{ml}$, for aPTT 0.1 aXa Units $/ \mathrm{ml}$ and for Heptest 0.05-0.1 aXa Units $/ \mathrm{ml}$.

Dept. of Internal Medicine, Division of Angiology, J.W.GoetheUniversity, T.Stern-Kai 7, 60590 Frankfurt am Main, FRG *Dept. of Haematology, Medical School, Bialystok, Poland 
PREVENTION OF VENOUS THROMBOEMBOLISM IN ELDERLY MEDICAL IN-PATIENTS: A DOUBLE BLIND COMPARATIVE STUDY OF UNFRACTIONATED HEPARIN $(2 \times 5000$ IU) AND ENOXAPARIN (20 MG).

J. F. Bergmann* for the Geriatric Enoxaparin Study Group, France

In a previous study Enoxaparin was more effective and as safe as a placebo in the prevention of venous thromboembolism in elderly medical in-patients.

The next step was to compare subcutaneous $20 \mathrm{mg}$ Enoxaparin $[\mathrm{E}]$ once daily versus $5,000 \mathrm{IU}$ unfractionated heparin [UFH] bid in a double blind, multicentre study, in 442 patients over 65 years (age mean $\pm S D: 83.2 \pm 7.2$ ) hospitalised for an acute medical disease inducing a recent ( $\leq 3$ days) immobilisation.

Patients were hospitalised e.g. for severe infection (28\%), heart failure $(20 \%)$, stroke $(9 \%)$, cancer $(7 \%)$.

Existence of deep vein thrombosis was investigated by using the fibrinogen leg scanning. In case of positive results an additional phlebography was performed. Clinical signs of pulmonary embolism had to be verified by either ventilation-perfusion lung scanning or angiography.

During the 10 days treatment period, among the 423 patients evaluable in intention to treat analysis, $20(4.7 \%)$ had a thromboembolic event: $10 / 207$ $(4.8 \%$ ) in the E group ( 9 isotopic venous thrombosis and one pulmonary embolism) and 10/216 (4.6\%) in UFH group (10 isotopic venous thrombosis).

As 3 patients did not received any injection, 439 patients were evaluable for safety: 216 for $E$ and 223 for UFH. In the E group one major and one minor haemorrhage (0.9\%) occurred compared to 2 major and 2 minor in the UFH group $(1.8 \%) .7$ patients $(3.2 \%)$ in the $E$ group and $8(3.6 \%)$ in the UFH group died. One sudden death occurred in each group without autopsy. None of the deaths was related to proven pulmonary embolism or haemorrhage. One asymptomatic unfractionated heparin induced thrombocytopenia was described.

Conclusion Enoxaparin $20 \mathrm{mg}$ once a day during 10 days is as safe and as effective as $5,000 \mathrm{IU}$ unfractionated heparin bid in the prevention of venous thromboembolism in medical immobilised elderly in-patients.

* Clinique thérapeutique, Hôspital Lariboisière, F-75010 Paris, France

\section{4}

IMPROVEMENT OF THROMBOSIS-PROPHYLAXIS IN PATIENTS HITH INTERNAL DISORDERS AND HIGH HEPARTMTOLERANCE gY ADAPTATION OF MEPARIN-DOSES TO SUITABLE PARAMETERS

J. Keute, I. Rohner, M. Maasberg, R. Seitz und R. Egbring

It is mell known since the early fiftieth that the response to heparin administration (heparin-tolerance) differs widely.

The hepar in level for therapeutical use range between 0,2-0,6 U HMHHepar in (HH:H) and the TT or aPTT may be twice to threefold prolonged.

with the thrombintitration according to J. Jürgens $(0,15-0,3-0,9$ u Thranbin/test) semi quantitativ aestimation of heparin response could be evaluated in patients receiving only low-dose HMuH prophylaxis, there the heparin levels "should possible be" between $0,05-0,2$ u heparin/mt plasma. In order to get a suitable hepar in response, higher doses than $5000 \mathrm{u}$ thult should be achinistered.

Even after $8000 \mathrm{U}$ the heparin level in $52 \%$ of the patients the heparin concentration found to lower then $0,05 \mathrm{u} / \mathrm{ml}$ HMLH $3.5 \mathrm{~h}$ after sc administration (low responder). Nearly $26 \%$ of the patients had levels between $0,05-0,15 \mathrm{U} / \mathrm{ml}$ HMHK (responder) and only a few patients were high-responder with vatues $>0,15 \mathrm{u} / \mathrm{mt}$ Hнин.

With the thrombintitration (thrombin/test lower than 0,3 U) similar results are obtained.

The results show that the so called low responder have a high heparintolerance and are insufficiently anticoagulated even for prophylaxis.

Patients with internal disorders tho are sometimes severely ill requires higher doses of heparin to obtain a "prophylactic" heparin $(0,05-0,2 \mathrm{U} / \mathrm{ml})$ level.

But higher doses of HMLH can oniy subcutaneously be acministrated if the concentration of heparin is as high as 40000/U HMHH/mL (Depot-Thronbophob). Furthermore the prophylaxis of thrombosis with HMLH in patients with severe internal disorders should not schematicalty be performed as in surgical patients. The response to hepar in should be evaluated and individual adaptation on the control paraneters for heparin will be much more effectic.

Philipps-University of Marburg, Department of Hematology/Oncology, Baldingerstrasse, 35033 Marburg, Germany
RISK OF THROMBEMBOLISM IN OUTPATIENTS WITH PLASTER CAST IMMOBILISATION - HOW IS THE DEPENDENCE OF RISK FACTORS TO BE DETERMINED?

U. Spannagel*, P. Kujath**, W. Habscheid***

*Würzburg, *t Lübeck, ***Ostfildern

The risk of thromboembolism in surgical outpatients with immobilisation in a non-weight bearing plaster cast has been so far only evaluated in few retrospective studies. Concerning the influence of additional risk factors (RF) in these patients, there are still no scientific findings.

In an open, randomized and prospective study, the incidence of deep vein thrombosis and the influence of additional RF in 253 surgical outpatients with plaster cast immobilisation of the lower extremity was determined. Of these patients, only 127 received a prophylaxis with low molecular weight heparin. Basic and anamnestic data were comparable in both groups. The average duration of immobilisation in the cast was 15.7 days.

After removal of the cast or in case of clinical suspicion of thrombosis compression ultrasound, and for confirmation, venography was performed.

Without prophylaxis 21 (16.5\%), with LMWH-prophylaxis only $6(4.8 \%)$ thrombosis occured $(2 p<0.01)$.

The severity of the injury was an essential RF. $29 \%$ of the patients with fractures treated in a non-weight bearing plaster cast developed without prophylaxis DVT. With LMWH the rate was only $10.3 \%$. Further important RFs was the age over 30 years as well as obesity and varicosis.

Patients without thrombosis had on the average $1.2 \mathrm{RF}$ while patients with thrombosis had $1.9 \mathrm{RF}$. Because almost half of all patients with thrombosis had none or only one RF, we conclude that a prophylaxis of DVT with LMWH in patients with plaster cast immobilisation should be administered also in the absence of RFs.

\section{6}

EFFECT OF PENTHOSAN POLYSULPHATE TREATMENT ON RESTENOSIS IN PATIENTS UNDERWENT PERCUTANEOUS TRANSLUMINAL CORONARY ANGIOPLASTY

F. Gûrtler, K. Várnai, and L. Szatmáry*

The insufficient fibrinolytic capacity is known to be a pathogenic factor in coronary artery disease.

Our aim was to determine the possible protective effect of penthosan polysulphate on restenosis. This study was carried out in 83 patients underwent one or more than one PTCA

Response for venous occlusion was determined on the first week after PTCA

in all patients. The venous occlusion test was carried out in the usual way (Petaja, at al. 1989) using t-PA and PAI KABI test kits. The threshold of normal response for venous occlusion was established empirically by 15 healthy persons. Poor responders were treated with SP54 for half a year and followed up nearly a five year period.

Beneficial effect could be observed in same rate in both groups, but the results showed no correlation between the changes of venous occlusion responses and the clinical outcomes.

Decreasing tendency or disappearance of reocclusion was observed using SP 54 during PTCA seems to be a new observation which is worth of mention.

*Present address: Cardiovascular Surgery Clinic of Semmelweis Medical University, Budapest

Department of Clinical Laboratory, Hospital of Hungarian State Railways,

Podmaniczky u. 111, H-1062 Budapest, Hungary 
187

THE EFFECT OF SODIUM PENTOSAN POLYSULFATE (SP54) INFUSION ON BLOOD COAGULATION PARAMETERS AND ON LIPID RISK FACTORS

K. Fendler and J. Szokola

$300 \mathrm{mg}$ of SP 54 in half a litre of salt solution were infunded in slow drops during 3 hours to persons suffering from peripheral artery and coronary diseases. At the end of the infusion, decrease of the euglobulin lysis time, prothrombin and fibrinogen values was observed in both groups. The atherogenic lipid factors moved optimally: the HDLC and the lipoprotein A, levels increased, and the triglyceride and lipoprotein B levels decreased. The observed and above-mentioned changes show that intravenous SP 54 could be effective in prevention of arteriosclerotic events. We widen our analysis with the observation of Iipoprotein $A$ while the role of lipoprotein A at CHD is more and more considered.

Central Laboratory of County Hospital Baranya, Garai str. 2, Po 172, Pécs, Hungary

CONTINUATION OF POSTOPERATIVE PROPHYLAXIS OF THROMBOSIS WITH CLEXANE 40 AS OUTPATENT TREATMENT FOLLOWING ACCDENT AND ORTHOPAEDIC SURGERY C. W. Flosbach*

In an earlier trial investigating the prophylaxis with $40 \mathrm{mg}$ Enoxaparin [E] (Clexane) od in orthopaedic inpatients, doctors stated that prolonged prophylaxis after discharge from hospital was needed in $15 \%$ of the patients. Therefore the aim of the present study was to investigate efficacy, safety, convenience of a prophylaxis of deep vein thrombosis [DVT] and pulmonary embolism [PE] with $\mathrm{E}$ after discharge from hospital following accident and orthopaedic surgery. Inpatient postoperative prophylaxis had to be started with $E$, the duration of the subsequent outpatient prophylaxis with $\mathrm{E}$ was at the clinician's discretion. DVT and PE were to be detected by clinical signs and whenever possible confirmed by phlebography.

Of 851 patients enrolled, 782 were evaluable with 752 having received outpatient prophylaxis. The mean duration of the inpatient prophylaxis was 2 weeks and of subsequent outpatient prophylaxis 3 weeks. 11 thromboembolic events were re ported $(1.4 \%)$ during the postoperative inpatient prophylaxis. During the outpatient prophylaxis with $\mathrm{E} 3 / 752$ patients $(0.4 \%)$ showed clinical signs of thrombosis confirmed by phlebography. The parients had at least 3 risk factors for thrombosis. Two of the patients had a fracture treated with osteosynthesis and 1 a rupture of Achilles' ligament treated with plaster cast. All three DVT developed between the 6th and 8th week after the start of treatment. In addition 2 DVT occurred 2 and 3 days after discontinuation of prophylaxis: 5 and 7 weeks after the injury. Both patients had a bone fracture treated with osteosynthesis and plaster cast respectively. 512/752 patients (68\%) applied the injections themselves. Of these more than $75 \%$ considered the application as easy/simple and over $85 \%$ stated acceptance as very good/good. Adverse events occurred in 4.3\% during outpatient prophylaxis being in majority reactions at injection site. 1 prolonged menstruation bleeding was documented. There was no case of thrombocytopenia reported.

Conclusion: Prolonged prophylaxis seems to be needed especially after bone fracture and ligament rupture treated with plaster cast and osteosynthesis. Late upcome of DVT in such patients might occur 5 through 8 weeks after injury. Enoxaparin $40 \mathrm{mg}$ od (Clexane) is a effective and safe prophylaxis in outpatients. Most patients can inject themselves and acceptance of self-injection is very high.

* Clinical Research Dept., Rhône-Poulenc Rorer, D-50829 Cologne

\section{9}

PREVENTION OF DEEP VEIN THROMBOSIS WITH INTERMITTENT SEQUENTIAL PNEUMATIC LEG COMPRESSION (ISC) IN PATIENTS UNDERGOING TOTAL HIP REPLACEMENT Adam O., Kormann B, Fisch R., "Wiseman M., Rheumaeinheit der LMU, Pettenkoferstraße 8a, 80336 München, "Leibniz Rechenzentrum, Barer-Straße 21, 80333 München.

Established measures to prevent deep-vein thrombosis comprise compression stockings, the application of low molecular weight heparin, maintenance of appropriate Antithrombin III levels, and physical therapy. With this regimen the incidence of DVT can be lowered after total hip replacement from approximately $40 \%$ to $10 \%$. We aimed to evaluate in a matched study the additional effect of ISC with a device provided by Kendall GmbH, Neustadt, Germany, on the incidence of DVT and pulmonary embolism in a cohort of high risk patients after total hip replacement.

Two groups of 50 patients (66.7 \pm 10.1 years) were matched according to age, sex, previous thrombosis, a score reflecting risk factors, the method of operation and anaesthesia, to establish two comparable cohorts. Both groups were given all measures for prophylaxis of DVT, according to a standardized protocol. Additionally. the intervention group was given ISC with the SCD system, Kendall. ISC was initiated immediately after hip replacement and continued for five days, from 2 p.m. to 8.00 a.m. every day. Before, 4 days after the operation, and after complete mobilization of the patient Duplex-ultrasound was performed and in questionable cases reevaluated by phlebography. Statistical analysis was performed by multivarate analysis of variance (SPSS MANOVA).

Both groups were comparable according to matching criteria and control parameters (PTT, ATIII, serum protein, hematocrit). The incidence of DVT was statistically significantly $(p<0.001)$ lower in the intervention group with ISC ( 1 DVT) compared to the control group (6 DVT and 1 pulmonary embolism).

Our results provide evidence that ISC can lower the incidence of DVT and pulmonary embolism in patients after total hip replacement.

TRANSPLACENTAL PASSAGE OF LOW MOLECULAR WEIGHT HEPARIN - A THIRD TRIMENON RANDOMIZED STUDY COMPARING ENOXAPARIN WITH UNFRACTIONATED HEPARIN S. Haas*, E. Halberstadt, K.T.M. Schneider, P. Berle, A. Stemberger, C.W. Flosbach

Unfractionated heparin (UFH) has been the drug of choice for the prevention and treatment of thromboembolic complications during pregnancy. Low molecular weight heparins (LMWH) have shown to offer several advantages over UFH, i.e. they cause less side effects and their prophylactic use requires only single daily injections. Since various preparations of LMWH are individual substances, the absence of transplacental passage has to be proven for each compound separately. In an earlier trial it has been shown that Enoxaparin (E) does not cross the placenta barrier during the second trimenon. The aim of the present study was to confirm these results within the third trimenon. 48 pregnant women admitted for caesarean section were included in the present trial. 24 received $20 \mathrm{mg}$ Enoxaparin s.c. 2.5 h before operation, and 12 women were treated with 5.000 IE UFH. In addition, 12 patients who did not require preoperative prophylaxis were untreated or received only postoperative injections. Women receiving preoperative UFH or E were randomly allocated to the treatment groups. Baseline blood samples were drawn from the mother before the preoperative UFH/E injection or for the non-treated women $2.5 \mathrm{~h}$ before caesarean section respectively. Immediately after delivery, blood samples were drawn from the mother and from the umbilical vein. As expected, the increase of anti Xa-activity in the maternal blood was more pronounced after injection of $\mathrm{E}$ than after UFH injection. In contrast, anti Xa-activity remained below the detection limit in samples drawn from the umbilical vein, indicating that no increase of activity occurred in the newborns of the three groups (UFH, $\mathrm{E}$, non-treated).

Conclusion: The results of the previous second trimenon trial and of this third trimenon study have provided evidence that a prophylctic dose of 20 mg Enoxaparin does not cross the placenta barrier and may be a safe prophylaxis of thromboembolism in the second and third trimester of pregnancy.

*Institut für Experimentelle Chirurgie der Technischen Universität München, Ismaninger Str. 22, D-81675 München 
191

The topical treatment of infusion thrombophlebitis with pentosan polysulfate sodium ointment - A randomised double-blind study

\section{Rozsos, L. Kollár, M.E. Scholz}

In a 7 day randomised double-blind trial, the equivalence of efficacy of pentosan polysulfate sodium ointment (PPS ointment, $0.5 \%$ ) in comparison to mucopolysaccharide ointment (MPS ointment, $0.445 \%$ ) was studied in 110 adult stationary patients suffering from acute infusion thrombophlebitis in one arm. The study medication was applied three times daily. Antithrombotic and antiinflammatory efficacy were evaluated by daily monitoring of the severity of the symptoms (induration, swelling, erythema, temperature, pain) as defined by scores on a five-stage scale. In addition, 125 I-fibrinogen was injected on the day before the onset of treament and radioactivity was measured over the inflamed veins on day 1,3 and 7 of the treatment period. Both ointments were equivalent in their efficacy. However, the symptom attenuation with the pentosan polysulfate sodium ointment appeared to be somewhat faster and, since this formulation is prepared from plant origin, this preparation was free from any risk of BSE infection. No adverse events were reported and the treatment was well tolerated by all patients.
193

SELECTIVE THROMBOLYSIS: TECHNIOUES, INDICATIONS AND RESULTS

L. Horváth, r. Battyăny, F. Meláth, G. Horváth Department of Radiology, University School of

Medicine, H-7624 PÉCS, Hungary

PURPOSE:There are non-organized clots in the lower linb arteriescanolicating or biniting atherosclerosis in aany cases. Acute occlusions are very urgent to be treated. Since throabi ere sujject to lysis (natural or arteficial) they siould first be attenpted to be treated by selective intravascular tirrombolysis.

MATERIAL AND METHOD: Selective thrombolysis was the choica of treatment in 74 jatients. The cathecer was aither imbadded or placed near to the clot for irore effective lysis. Hecianical clo: fragrentation, throinbus aspirasion and clot infiltration aere integral parts of selective clot lysis. 5treptokinase was infused in sl patients and urokinase in 13 of them. There was no significant difference in effectivity or complication rate between both drugs. The postthroabolytic medication is vital in the long-tern patency. Dicumarol orfand oral fibrinolytic stimulants as pentosan polysulphate proved to be the most effective in this respect.

RESULTS: From 74 patients 59 benefitted by a successful lysis and 10 by a partial lysis. In 5 patients the treatnent was unsuccessful. In 56 of 59 successful cases pentosan polysulphate was infused additionally to the lytic agent. In the partially successful 10 cases only 3 and in the unsuccessful group only $l$ patient received the additional drug.

Conclusion: The betiod is a very fine example of top interventional radiological procedure needing expertise in catheter manipulation. in clinical medicine, pharacology and laboratory topics.

\section{4}

ORALLY INDUCED THROMBO-ATHEROLYSIS FOLLOWING TRANSLUMINAL ANGIOPLASTY AND SELECTIVE CLOT LYSIS

L. Horváth, I. Battyány, J.-J. Pinot and M. Maynar

PURPOSE: In the future radiologists Hill be able to maintain their interventional practice only if their long-teria results will be conparable to those of the vascular or general surgeons. To achieve this goal radiologists aust find sone new ways of beeping the recanalized arterial lumen patent: for a long time.

MATERTAL AND METHOD: Data of 760 patients have been analized regardiag the reginen, follow-up results and individual drugs. Complaits, walkino distance, Doppler index, duplex sonography in some cases, palpation and auscultation have been regularly performed by experineced doctors. Adjuvant tinerapy was based on several factors including: age, coexisting disease, diabetes, hematocrit, saruil cholasterol, plasma fibrinogen and blood viscosity. Of course the present vascular status was the most determinig factor in selecting the medication. The most effective drugs were pentosan polysulphate and dicumarol regarding the long-teri patency.

RESULTS: A paralel group of patients were evaluated alternatively receiving dicuarol or pentosan polysulphate. The 5 year patency rate was $58 \%$ and $76 \%$ respectively. The fibrinclytic ef fect of pentosan polysulphate did even further improved the recanalized and dilated lumina in iajority of cases.

CONCLUSION: "Why to recanalize if the lumen $c$ an not be savad?" 
RELATIONSHIP BETUEEN HEMOSTATIC OISORDERS AND SIGNS OF BLEEDING FOUND AT AUTOPSY IN 36 PATIENTS MITH SEVERE INFECTIONS

U. Busch, L. Lerch, M. Maasberg, R. Seitz, T. Saldeen, R. Wallin, R. Egbring

Based on autopsy findings, 36 patients with severe infections, on whom coagulation studies had been performed before death, were divided into two groups with pronounced or less signs of bleeding. Hemorrhage frequently was serious and in 10 patients it was regarded as one of the lethal compl ications.

In this retrospective study Quick value, thrombocyte count, thrombin-antithrombin III (TAT), neutrophil elastase-alpha, antitrypsin (ELP- ${ }_{1} \mathrm{AI}$ ) and the elastasespezific fibrin degradation products $F P B$ 30.43 were measured in time course before death.

On admission TAT level was elevated in both groups showing enhanced thrombin generation. Undergoing substitution with antithrombin III concentrate and fresh frozen olasma both groups showed decreasing Tar levels before death. Dic was favorably influenced by substitution therapy. In contrast to this in the group presenting more bleding signs thrombocyte count decreased and $\mathrm{FP} / \mathrm{A}_{30-43}$ level increased in the last three stages before death. At the endpoint the thrombocyte count was highly signifieant lower $(p<0,0043)$ and the $F p B 30-43$ level highly significant higher $(p<0,0010)$ in the patients presenting pronounced signs of bleeding. If the capacity of specific inhibitors $\left(K_{1} A T, \alpha_{2}{ }^{n}\right)$ is consumed in advanced stages of septicenia, neutrophil elastase could contribute to hemorrhegic disorders by eleaving elotting factors and inhibitors directly, by altering of endothelial cell surface and by inducing of fibrinogen receptor exposure with consecutive platelet aggregetion by fibrinogen.

In conclusion, the data suggest that lethat bleeding may be caused by drop of thrombocyte count and proteolytic action of neutrophil elastase.

Philipps-University of Marburg, Department of Hematology/Oncology, Baldingerstrasse, 35033 Marburg, Germany

Department of Forensic Medizin, University Uppsala, Sverige

\section{Paediatric Haemostasis}

196

Homozygous Antithrombin-III-Deficiency Causing Severe Arterial and Venous Thrombqembolism in Yery Early Childhood S.Gandenberger-Bachem, A.F lemmer , T.Meitinger ${ }^{2}$, V.Chawdhury , S.L. Thein , K.Auberger

'Children's Hospital in the Dr.von Haunersches Kindęrspital and ${ }^{2}$ Cenetic Institute of the University of Munich, Institute of Molecular Medicine, John Radcliffe Hospital, Dxford

Homozygcus antithrombin-III-(AT-III)-deficiency is rare. We know reports about 9 cases, which were associated with a hich incidence of thrombosis often including the arterial vessels. At the age of 14 months a turkish girl first presented to hospital with acute pelvic vein thrombosis. Also, hemioaresis had been noted since the age of 5 months. Cerebral mangetic resonance imaging (ARI) showed a defect of $6 \times 7 \times 4 \mathrm{~cm}^{3}$ in the region supplied by the left arteria cerebri media and $M R-$ angiography showed the discontinuance of branches of this artery. Abdominal ultrasound demonstrated a calcification of the intrahepatic vena cava; in addition, in abcominopelvic MRangiooraphy flow sionals were missinc in the distal vene cava inferior and in both venae iliacae internae. These findings were interpreted as evidence of recurrent thrombosis. Plasma AT-III-activity in our patient was permanently decreased to levels between 27 and 50\% of normal. In her asymptomatic and reportedly unrelated parents levels were found between 40 and $67 \%$ and in both brothers approximately 100\%. By genetic analysis the underlying mutation was identified as 99 Leu to Phe (CTC to ITC); both parents were heterozygous for this mutation whereas our patient was homozygous. Crossed immunoelectrophoresis demonstrated reduced heparin binding of this AT-III-variant.

This is the tenth reported case of homozygous AT-III-deficiency and the seventh with abnormal heparin binding. In accordance with other reports we found severe thrombophilia including the arterial vessels and occuring already in very early childhood.

\section{7}

VON WILLEBRAND FACTOR IN NEONATES AND IN YOUNG INFANTS.

KB.Thomas, AH. Sutor, A. Zehenter, A. Grohmann, N. Altinkaya, J. Leititis

Plasma von Willebrand factor (vWF) was examined as part of preoperative screening from three groups of infants aged 2-6days, 8-30days and 30days6 months. None of the patients had haemostatic abnormalities. The vWF Antigen (AG) and vWF-Collagen binding activity (CBA) were measured by an ELISA. The CBA represents a functional assay for $\mathrm{VWF}$ which correlates well with Ristocetin Cofactor Assay. The results were compared to normal adult plasma pool and expressed in arbitary units/ml. Median and 25-75\% range are shown.

\begin{tabular}{|c|c|c|c|}
\hline Age/Parameter & AG $(\mathbf{U} / \mathbf{m l})$ & $\mathrm{CBA}(\mathrm{U} / \mathrm{ml})$ & CBA/AG \\
\hline $\begin{array}{l}2-6 \text { Days } \\
(\mathrm{n}=43)\end{array}$ & $\begin{array}{l}1.65 \\
(1.20-1.97) \\
\end{array}$ & $\begin{array}{l}2.09 \\
1.67-2.83) \\
\end{array}$ & $\begin{array}{l}1.25 \\
(1.04-1.55) \\
\end{array}$ \\
\hline $\begin{array}{l}\text { 8-30 Days } \\
(\mathrm{n}=11)\end{array}$ & $\begin{array}{l}1.35 \\
(1.19-2.24)\end{array}$ & $\begin{array}{l}1.73 \\
(1.30-2.4)\end{array}$ & $\begin{array}{l}1.18 \\
(0.91-1.46)\end{array}$ \\
\hline $\begin{array}{l}\text { 1-6 Months } \\
(n=10)\end{array}$ & $\begin{array}{l}1.17 \\
(1.03-1.57)\end{array}$ & $\begin{array}{l}1.24 \\
(1.17-1.40)\end{array}$ & $\begin{array}{l}1.04 \\
0.88-1.15)\end{array}$ \\
\hline $\begin{array}{l}>20 Y \text { Adults } \\
(n=19)\end{array}$ & $\begin{array}{l}1.09 \\
(0.92-1.25)\end{array}$ & $\begin{array}{l}1.20 \\
(0.98-1.27)\end{array}$ & $\begin{array}{l}1.08 \\
(0.97-1.16)\end{array}$ \\
\hline
\end{tabular}

The $A G$ and $C B A$ were highest in the newborn group and these values decreased progresively with age, reaching adult range between 1-6th month. Similarly, CBA/AG ratio, which reflects the functional integrity of the $\mathrm{vWF}$, was higher in newborns and reached normal adult level by 6 th month. The vWF multimer showed an increased amount of the protomer in the 2-6 day group; it gradualy became similar to that of normal adults by the 6th month. Our results show high levels of vWF-AG and CBA in newborns as compared to adults. Diagnosis of vWD performed on very young infants should be confirmed after the 6th month of life. It is conceivable that the high level of vWF in newborns could compensates for the generally hypofunctional platelets.

Universität-Kinderklinik, Mathildenstraße 1, D-79106 Freiburg. 
Protein $\mathrm{C}$ and protein S: Comparison of activity and antigen levels in healthy children ${ }^{1} \mathrm{U}$. Nowak-GöttI, ${ }^{3} \mathrm{~B}$. Zwinge, ${ }^{2} \mathrm{M}$. Funk, ${ }^{4} \mathrm{~V}$. HachWunderle, ${ }^{2}$ W. Kreuz and ${ }^{3}$ I Scharrer

In 122 healthy children (6 months - 16 yrs) PT, protein $C$ and protein $S$ were studied before elective surgery. Blood samples, obtained by venipuncture were directly placed into premarked plastic tubes (citrate $3.8 \%$ / blood $1: 10$ / Saarstedt ${ }^{R}$, centrifugated at $4^{\circ} \mathrm{C}$ for $20 \mathrm{~min}$ at $3000 \mathrm{~g}$ and frozen at $-80^{\circ} \mathrm{C}$. Coagulation studies were investigated in serics no later than 6 months. PT was measured with IL Test TM (PT - fibrinogen HS/ IL), protein $\mathrm{C}$ activity with Coamate ${ }^{R}$ Protein $\mathrm{C}$ (Chromogenix) and protein $\mathrm{S}$ with IL Test TM (Instrumentation Laboratory) on an ACL 300 R. Antigens were investigated by Laurell electrophoresis (PC - antigen: Amerian Diagnostica, Ch. 92-06, total PS: Stago Diagnostica, Ch 924055). Results in $\%$ of normal (upper and lower boundaries, encompassing $95 \%$ of the paediatric population):

\begin{tabular}{l|cccc}
\multicolumn{1}{c}{$6 / \mathbf{1 2}-\mathbf{2 . 5}$ yrs } & $\mathbf{2 . 6}-\mathbf{7}$ yrs & $\mathbf{8}-\mathbf{1 6}$ yrs & adults \\
\hline PT & $78-100$ & $78-100$ & $77-100$ & $75-100$ \\
\hline PC act & $53-91$ & $64-110$ & $64-110$ & $70-130$ \\
PC ag & $64-97$ & $70-113$ & $68-110$ & $74-150$ \\
\hline PS act & $44-124$ & $59-136$ & $70-125$ & $60-140$ \\
PS ag & $63-136$ & $82-150$ & $82-141$ & $65-140$
\end{tabular}

Age related significant correlations were only found in children aged 6 months to 2.5 years $(\mathrm{p}<0.02)$ in protein $\mathrm{C}$ activity and antigen. In 66 children activities and antigens were investigated simultaneously: we found no correlation between activity and total protein $\mathrm{S}$, whereas protein $\mathrm{C}$ antigen and activity levels showed a significant positiv correlation $(\mathrm{p}<0.02)$.

Dept. of ${ }^{1}$ Paediatric Haenatology $/$ Oncology, University of Münster, Albert - Schweitzer - Str. 33, 48149 Münster

Dept. of ${ }^{2}$ Paediatrics and ${ }^{3}$ Internal Medicine, University of Frankfurt Main, ${ }^{4}$ William -Harvey Clinic, 61231 Bad Nauheim

\section{9}

VITAMIN K DEFICIENCY IN EARLY INFANCY - RELATIONS TO NEONATAL VITAMIN K PROPHYLAXIS

$H$. Lenk and $F$. Kertzscher

Severe coagulation defects caused by vitamin $K$ deficiency were diagnosed in 16 newborns and infants over a period of about 12 years.

Seven children where completely breastfed and admitted to the hospital for coagulation prablems. The coagulation disorder was also discovered in 9 hospitalized patients. 0rally they got exclusively human milk but additional infusions depending on the underlying disease. Secondary diseases may have contributed in nearly two thirds of the cases to the expression of the coagulation defect. Parenteral administration of vitamin $K$ normalized the depressed factors of the prothrombin complex in a very short time.

The manifestation period of the bleeding disorder ranged fram the 2. up to the 73 . day of life. After introduction of general vitamin $K$ prophylaxis in 19876 further cases where registered, but only one without a second disease.

Cases observed after introduction of oral prophylaxis remember, that the mode of application has to be changed, when the vitamin $K$ resorption may be influenced by underlying diseases.

Department of Pediatrics, University of Leipzig DststraBe 21-25, D - 04317. Leipzig, FRG.
Deep Vein Thrombosis In A 12 Year Old Girl With Antiphospholipidantibodies

E. Lenz, D. Klarmann, S. Becker, I. Martinez, I. Scharrer, W. Kreuz

Thrombosis does rarely occur in childhood and is always a reason to do a meticulous assessment of the coagulation system.

We would like to report a case of a 12 year old patient with deep vein thrombosis and associated antiphospholipidantibodies, where we suspect development of an autoimmune disease.

Risk factors predisposing to develop thrombotic complications could be ruled out in this patient. Patients past medical history and family history were unremarkable regarding hypercoagulability. Screening for thrombosis predisposing coagulation parameters, such as Antithrombin III, Protein $\mathrm{C}$ and etc. were in normal range.

Lupus anticoagulant was detected and confirmed in the KCT (Exnertest), DRVVT and mixing studies. ELISA for anticardiolipidantibodies was positive.

In literature occurence of a thrombotic event in association with a lupus anticoagulant is determined to be as high as $30 \%$. Antiphospholipidantibodies are known to occur in connection with autoimmune disease, especially systemic lupus erythematodes, lymphoproliferative disease, viral and bacterial infections and drug-associated.

The investigations in our patient showed positive titers for ANA, dsDNSantibodies, Ro/SSA, a positive Coombs-test, elevated ESR and a temporary thrombocytopenia. Titers for $\mathrm{IgA}$ and $\mathrm{IgE}$ were above normal range. Physical examination especially regarding dermatologic and neurologic symptoms of SLE was without pathological abnormality. Kidney function tests, chest-x-ray, examination of the joints were normal.

In this patient SLE has to be suspected, but at time only 3 out of 4 ARAcriteria, needed to make the diagnosis of SLE, are fullfilled.

It has been reported, that in some cases a thrombotic event with Antiphospholipidantibodies has proceeded the diagnosis of SLE for years.

Venous thrombosis in connection with antiphospholipidantibodies demands a strict oral anticoagulation and can be necessary lifelong, if high APA-titers persist.

Department of Pediatrics, Universityhospital Frankfurt, Theodor-Stern-Kai 7, 60596 Frankfurt

201

\section{SURGERY IN A 4-YEAR-OLD BOY WITH F XI-DEFICIENCY BLEEDING PROPHYLAXIS WITH A VIRUS INACTIVATED F XI-CONCENTRATE \\ C.Escuriola, I.Martinez, S.Becker, E.Lenz, D.Klarmann, W.Kreuz}

Congenital Factor XI-deficiency is an uncommon bleeding disorder occuring mainly in the Jewish population. It is characterized by a variable and unpredictable bleeding tendency which may occur in mild (F XI-activity $>20 \%$ ) and severe deficiency (F XI-activity $<20 \%$ ).

As spontanous hemorrhage is unusual, patients mainly present with troublesome and sometimes dangerous postoperative or posttraumatic bleeding, especially after surgery in squamous tissues.

In the past fresh frozen plasma was the only effective treatment in case of bleeding carrying the risk of virus transmission.

Since 1992 a F XI-concentrate is available (Bio products, Herts, UK) being dry heated at $80^{\circ} \mathrm{C}$ for 72 hours.

We would like to report the successful bleeding prophylaxis with $F$ XI-concentrate in an urological surgery procedure in the case of a 4 . year-old boy of Jewish origin suffering from a congenital severe $F$ XI-deficiency with a residual activity of $4 \%$. F XI-deficiency was detected in the asymptomatic patient after birth because of a prolonged aPTT. Decreased F XI levels of the mother $(60 \%)$ indicates an inherited disease.

Because of severe phimosis the patient had to undergo circumcision at the age of 4 years.

By protective substitution of Factor XI-concentrate (Bio products) the surgery could be carried out without any bleeding complications. Side effects could not be observed.

According to the ICTH-criteria for the safety of clotting factor concentrates the patient was monitored with no evidence for transmission of HIV or hepatitis viruses.

Department of Pediatrics, University Hospital Frankfurt, Theodor-Stern-Kai 7, 60596 Frankfurt 


\section{Severe Factor VII-Deficiency In A 15-Year Old Boy}

E. Lenz, D. Klarmann, C. Escuriola, I. Martinez, S. Becker, W. Kreuz

Hereditary factor VII-deficiency is a rare bleeding disorder with an estimated incidence of 1:500 000. Approximately 150 severe cases are reported. It is inherited as an autosomal-rezessive trait.

Homozygous may have bleeding problems, severity of which does not always correlate with factor VII-levels. However, patients with less than 1-2 \% of factor VII-activity frequently have bleeding manifestations similar to those seen in severe classic hemophilia (Robertson et al. 1992).

We would like to report the case of a 15-year old boy with homozygous factor VII-deficiency and a factor VII-residual activity of less than $2 \%$, who has been followed up in our out-patient clinic since 1984.

Bleeding symptoms in this patient included insatiable nosebleeds and prolonged bleeding after tooth extraction.

Bleeding events are successfully treated by a factor VIIconcentrate (factor VII STIM 4 Immuno). Dental surgery could be performed under protection of this concentrate without bleeding complications.

The patient is substituted prophylactically twice a week. Interruption of this prophylactical regimen leads to frequent epistaxis which is difficult to controll. Under regular infusion of factor VII-concentrate bleeding symptoms can be reduced to a minimun and can be treated with single infusions.

Department of Pediatrics, University Hospital Frankfurt, Theodor- Stern- Kai 7, 60596 Frankfurt

\section{3}

HOMOZYGOUS PROTEIN C- DEFICIENCY IN A 2 YEAR OLD GIRL ASPECTS OF LONG- TERM TREATMENT

T. Beeg, D. Mentzer, l. Scharrer, W. Kreuz

The homozygous protein C-deficiency is one of the most rarely congenital thrombophil coagulation disorders. Since the first description in 1983 by Branson et al 17 cases were reported. Protein $C$ is a vitamin $K$-dependent coagulation factor which is produced in the liver. Protein $\mathrm{C}$ acts through inhibition of activated factors V and VIII and the platelet-associated factor $\mathrm{V}$ - factor $\mathrm{X}$-complex. In addition protein $\mathrm{C}$ has profibrinolytic properties. Through inhibition of plasminogen activator inhibitor I the concentration of plasminogen activator and plasminogen is elevated. Patients with the homocygous form of Protein C- deficiency are developing $3 \mathrm{~h}-5 \mathrm{~d}$ postpartum a "purpura fulminans" with generalized micro- and macrothrombosis with high mortality.

Our patient was born spontaneously after uneventful pregnancy. $12 \mathrm{~h}$ after birth she developed purpura fulminans with generalised thrombosis. She was initially treated with protein $\mathrm{C}$ - containing fresh frozen plasma ( ffp) and prothrombin complex concentrate ( PPSB). After diagnosis of homocygous protein $\mathrm{C}$ deficiency she received daily a high purified protein $\mathrm{C}$ concentrate for about 5 months. Then we started a long term anticoagulation with coumarins. During a viral infection with temperatures up to 41,3 ${ }^{\circ} \mathrm{C}$ she developed again clinical signs of a purpura fulminans. Therefore we initiated a combination therapy with coumarins and protein $\mathrm{C}$ concentrate. Coumarin therapy was monitored by prolongation of PT, protein $C$ concentrate was given every 3 days in a dose of $110 \mathrm{IU} / \mathrm{kg}$ bw. Since initiation of combination therapy we did not see any relapse of purpura fulminans for now 17 months. Statomotorical and psychological development of our patient correspond to that of normal children of the same age. In our opinion the combination of coumarins and protein $\mathrm{C}$ concentrate is a very succesful way to treat patients with protein $\mathrm{C}$ - deficiency. On the basis of high frequent substitution we are able to control PT very closely. In addition protein $\mathrm{C}$ substitution gives a supplementary protection for at least 3 days. Without side effects like antibody formation against protein $\mathrm{C}$ or adverse effects to coumarins our patients life expectancy might be good. Department of Pediatrics, Universityhospital Frankfurt. Theodor-Stern-Kai 7, 60596 Frankfurt

\section{CLINICAL COURSE AND DIAGNOSTIC EXAMINATIONS IN A CHILD WITH HOMOZYGOUS FACTOR V DEFICIENCY \\ D. Klarmann, S. Ehrenforth*, I. Scharrer*, B. Zabel**, J. Murray***, W. Kreuz}

Congenital factor $V$ deficiency (parahaemophilia) is a very rare, mainly autosomal recessive inherited coagulation disorder, first described by Owren in 1947. The male propositus of the present report was the first child of healthy, nonconsanguinous parents. The family history was uneventful with no history of bleeding diathesis. The child was born at term after an uneventful pregnancy by forceps delivery. Four days post partum, a prolonged bleeding time after venipuncture, anaemia (haemoglobin $7 \mathrm{~g} \%$ ), abnormal PT (INA: 4,8) and PTT (> 120 sec.) were found. Further investigations revealed not measurable FV antigen and activity. On day 7 p.p. neurological symptoms were conspicuous. A cranial ultrasound scan revealed a large subdural haematoma with shifting of the midline. Because no commercial FV concentrate is as yet available, replacement therapy with fresh frozen plasma (FFP) was initiated with a dose of $20 \mathrm{~m} / \mathrm{kgbw}$, followed by $2-6 \mathrm{ml} / \mathrm{kgbw}$ every 12 hours. Surgery was performed and the large subdural haematoma was removed without postoperative bleedings. Until the age of 6 months the child has remained clinically asymptomatic without any neurological sequelae or need for FV substitution. However, until the age of 2 years multiple haematomas, recurrent sustained mucous bleedings have occured spontaneously or after mild trauma and required FFP substitution on two occasions. The parents of the child were heterozygous for the defect with a similar decrease in FV activity $(35 \% / 43 \%)$ and antigen $(32 \% / 38 \%)$. To visualize the $\mathrm{FV}$ and also to examine cleavage patterns after activation, western blot analysis was done with and without activation by thrombin. The FV single chain was seen in both parents whereas no single chain was identified in the patient. The FV cleavage pattern of the parents resembled that of the control. This suggests that the FV produced by the parents activates correctly. The lack of any single chain or degradation products in the patient's plasma suggests that this child is either incapable of synthesizing FV or that any FV protein produced is rapidly degraded.

Depts. of Pediatrics and Internal Medicine

Johann Wolfgang Goethe Univesity, 60590 Frankfurt am Main, Germany

Dept. of Paediatrics, Johannes Gutenberg University Mainz**

Dept. of Biochemistry, University of Vermont***
205

PLATELET AGGREGATION IN WHOLE BLOOD AND IN PLATELETRICH-PLASMA IN PATIENTS WITH THROMBOCYTOPATHIES AND WITH VON WILLEBRAND DISEASE.

A. Zehenter, KB. Thomas, B. Zieger, AH. Sutor

We have compared platelet aggregation in whole blood (WB) and in platelet rich plasma (PRP). Investigated were two patients with Hermansky-PudlakSyndrome, one patient with Glanzmann's thrombasthenia and 19 patients with von Willebrand disease (wWD). Diagnosis of vWD was based on the family history, clinical symptoms and von Willebrand Factor analysis, including the multimer pattern.

In the thrombocytopathies a reproducible abnormal "zig-zag" pattern was shown in WB aggregation using ristocetin, while the results in PRP were normal. This characteristic pattern in WB was similar to that found in normal persons after aspirin ingestion. The aggregation extent with collagen was abnormal both in WB and in PRP.

In patients with vWD (15/19) the extent of platelet aggregation with ristocetin was unexpectedly normal both in WB and in PRP. In the remaining four patients with vWD the extent of the platelet aggregation was abnormal in PRP but not in WB. In contrast to the platelet aggregation extent in WB, the reaction time (the time taken from ristocetin addition to the start of platelet aggregation) was found to be a more sensitive diagnostic parameter. The reaction time was prolonged in 13 of 19 patients whereas in PRP the reaction time was always normal.

In conclusion, WB aggregometry represents a more physiological situation than PRP, incorporating leucocytes, erythrocytes and platelets and taking into account both platelet adhesion and aggregation. WB platelet aggregation provides additional information not always obtained with PRP and would be recommended as a suitable test in patients with suspected thrombocytopathy or VWD.

Universität-Kinderklinik, Mathildenstraße 1, D-79106 Freiburg. 
Evidence of activated coagulation in children with ALL treated with Prednison/L-Asparaginase in the ALL BFM 90 study. ${ }^{1} \mathrm{U}$. Nowak-Gött1, ${ }^{2} \mathrm{D}$. Schwa-

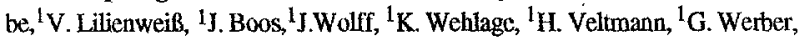
${ }^{1} \mathrm{H}$. Pollmann, ${ }^{2}$ W. Kreuz , ${ }^{2}$ B. Komhuber and ${ }^{1} \mathrm{H}$. Jürgens

In a prospective longitudinal study paramelers of coagulation and fibrinolysis (vWF:Ag, fibrinogen, antithrombin III, protein C, protein S, plasminogen, $\alpha_{2}$-antiplasmin, PAI activity, t-PA antigen, prothrombin Fl+2, D-dimer) were investigated in 47 children suffering from acute lymphoblastic leukemia, treated in the ALL-BFM 90 study (protocol I part I:prednison $60 \mathrm{mg} / \mathrm{m}^{2}$ day $1-29 ;$ E. coli asparaginase $10000 \mathrm{U} / \mathrm{m}^{2}$ day $12,15,18,21,24,27,30,33$; vincristine $1.5 \mathrm{mg} / \mathrm{m}^{2}$ and doxorubicin $30 \mathrm{mg} / \mathrm{m}^{2}$ day $\left.8,15,22,29\right)$. Between day 27 and 30 seven patients developed thrombotic events (V. subclavia $\mathrm{n}=4$, perforative ulcus ventriculi $\mathrm{n}=2$ (microvascular thrombi), sinus durae matris $\mathrm{n}=1$ ) and one patient suffered from intracranial bleeding. According to literature $(1,2)$ in the longitudinal follow up patients developed at III and plasminogen type I deficiency. In addition, most children showed a decrease of fibrinogen $<50 \mathrm{mg} / \mathrm{dl}$ (Clauss method) togehter with normal (!) values for PT, aPTT and a slight increase of TT. In good correlation to aPTT, TT, to enhanced $\mathrm{F} 1+2$ and d-dimers values (ELISA:Behring Werke/Marburg) the same patients showed 50 - $100 \%$ higher fibrinogen levels in derived fibrinogen (ACL). In opposite to these findings prior to the thrombotic event four patients showed a qualitative (type II) fibrinogen deficiency, normal antigen (RID and nephelometry: Behring Werke/Marburg), decreased functional activity (Clauss and derived method). In addition, all patients with thrombosis showed signs of severe endothel -cell damage with low or normal t-PA antigen, high PAI activity, high levels of VWF:Ag. In summary: without clinical relevance multiple coagulation changes occure in children with ALL treated with Prednison $/$-Asparaginase in protocol $\mathrm{VI}$. Severe bleeding was observed in $2 \%$ and thrombosis occured in $15 \%$. In these patients "Dysfibrinogenaemia" could be one of the release mechanisms for thrombosis togehter with local coagulation activation (Broviac catheter $\mathrm{n}=3$ ) with enhanced thrombin generation and signs of endothelial dysfunction (elevated WWF:Ag and PAI).

(1) Sutor et al (1992) Klin Pädiatr 204: 264-273

(2) Leone et al (1993) Thromb Haemostas 69: 12 - 15

Dept of Pediatric Haematology and Oncology, University Hospital Münster and Frankfurt am Main, Albert-Schweitzer - Str. 33, 48149 Münster
HEMOSTASIS IN SEVERELY HEAD INJURED CHILDREN - A CORRELATION OF BLOOD / PLASMA PRODUCT REQUIREMENT AND CLINICAL OUTCOME S. BECKER, W. SCHNEIDER, W. KREUZ, U. NOWAK-GOTTL*

After severe cranio-cerebral trauma defined by Glasgow-Coma-score (GCS) $<10$ parameters of coagulation and fibrinolysis (platelet count, vWF, F VIII:C, fibrinogen, AT III, protein C/S, plasminogen, D-dimer, t-PA-antigen, PAI-1 and $\alpha$-2-antiplasmin) were measured in 27 children (aged16 months to 15 years) initial and in the follow-up.

Changes in hemostasis were correlated with requirement of FFP, PCC, AT III concentrate, support of catecholamins and outcome. Results are summerized in the tables:

table 1: comparison of children with DIC and without DIC (U-test MANN-WHITNEY)

\begin{tabular}{|c|c|c|c|c|c|}
\hline valu & $\begin{array}{l}\text { vWF } \\
0.024\end{array}$ & $\begin{array}{l}\text { plasminogen } \\
0.02\end{array}$ & $\begin{array}{l}\alpha-2-\mathrm{AP} \\
<0.005\end{array}$ & $\begin{array}{l}\mathrm{t}-\mathrm{PA} \\
0.015\end{array}$ & \\
\hline & FFP & $\begin{array}{l}\mathrm{PCC} \\
<0.05\end{array}$ & concentrate & dobuta & \\
\hline
\end{tabular}

table 2: correlation with outcome (SPEARMAN Rank contelation coefficient)

platelets fibrinogen AT III PS D-dimer $\alpha$-2-AP PAI-1

$\begin{array}{llllllll}\text { corr.coeff. } & 0.678 & 0.606 & 0.496 & 0.539 & -0.642 & 0.538 & -0.711\end{array}$ GCS DIC FFP PCC ATIII cone. dobutamin

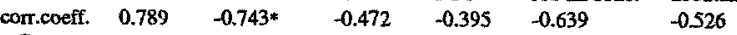

* Considering the number of pathological DIC defining coagulation tests the corr. coeff. was even higher $(-0,873)$

Continously enhanced D-dimer levels showed negative correlation to outcome (corr. coeff. -0.698)

AT III, fibrinogen and requirement of PCC and AT III concentrate were significantly different between patients with cranio-cerebral trauma $(\mathrm{n}=14)$ and patients with additional multiple trauma $(n=13)$.

The relationship between DIC following head injury in children and outcome is impressive: 15 children developed DIC, 8 of them died and two showed severe disability. 6 patients showed moderate disability, 3 of them suffered from DIC. 6 children had in sequela no handicap ( 2 with DIC) and only five children recovered completely (no case of DIC).

Centre of Pediatrics, Johann Wolfgang Goethe-University, Theodor-Stem-Kai 7, 60590 Frankfurt; *Centre of Pediatrics, University-Clinics, Münster; Germany

\section{Molecular Biology}

208

QUANTITATIVE DETERMINATION OF GLYCOPROTEIN Ib (GPIb) MRNA IN HUMAN PLATELETS

B. Pötzsch, L. Pichl, S. Schüpphaus, and G. Müller-Berghaus

Quantitative determination of mRNA in human platelets should be a useful tool in the diagnosis of inherited platelet disorders and could facilitate the investigation of the underlying molecular defects. An approach combining reverse transcription PCR with a colorimetric assay for quantitation of PCR products has been developed. As an example of the utility of the newly developed approach to quantify mRNA, the concentrations of MRNA coding for the GPIba and GPIbB subunit in normal human platelets were determined. Total RNA was isolated from washed human platelets using the guanidium-thiocyanate method and poly-A RNA prepared by chromatography on oligo(dT) cellulose ( $12 \pm 2 \mu \mathrm{g}$ poly-A RNA per $10^{11}$ platelets). First strand CDNA used for PCR amplification was synthesized using oligo(dT)primers and reverse transcriptase. DNA contamination of the isolated RNA was ruled out and a total number of $10^{9}$ platelets was found to be sufficient for generation of first strand DNA for PCR amplification. A 598 bp GPIb $\alpha$ CDNA fragment and a $738 \mathrm{bp}$ GPIbB CDNA fragment were amplified using two sets of primers. Each consisting of a biotinylated sense and an antisense primer bearing the sequence $5^{\prime}$-GGATGACTCA-3'. Through this sequence $P C R$ products specifically bind to the DNA binding protein GCN-4 coated on the wells of a microtiter dish. Subsequently, the amount of captured DNA was detected by streptavidin-peroxidase and quantified colorimetrically. The amount of target DNA was calculated using a calibration curve obtained with cloned GPIb $\alpha$ and GPIbB CDNA. In normal human platelets GPIb $\alpha$ and GPIbB mRNA were found in equimolar concentrations of $0.3 \pm 0.05 \mathrm{nmol}$ and $0.29 \pm 0.05 \mathrm{nmol} / 10^{10}$ platelets, respectively. These results are in accordance with previously published data showing identical concentrations of GPIb $\alpha$ and GPIbB molecules in. Most importantly, however, the data presented demonstrate that the described approach allows quantitative determination of platelet mRNA and, thus, offers a new tool in the diagnosis of inherited platelet disorders with quantitative deficiences. Hemostasis Research Unit, Kerckhoff-Klinik, Sprudelhof 11, D-61231 Bad Nauheim
209

EVALUATION OF A COLORIMETRIC ASSAY FOR DETECTION OF PCR-AMPLIFIED PRODUCTS

L. Pichl, K. Jäger, G. Müller-Berghaus, and B. Pötzsch

Clinical and large scale experimental applications of the PCR reaction require a sensitive, reliable, and easy method for detecting PCA products. A colorimetric assay allowing quantitative determination of amplified DNA based on the data published by Kemp et al. (PNAS, 86: 2423-2427,1989) was established. Sensitivity, specificity, and reproducibility of the assay were analyzed using a 1074 bp long glycoprotein Ib (GPIb) CDNA fragment inserted into the vector pBluescript $\mathrm{SK}^{+}$as target DNA. PCR amplification was performed using a biotinylated sense primer and an antisense primer bearing the sequence 5'-GGATGACTCA-3' specific for interaction with the DNA binding protein $\mathrm{GCN}-4$. Using this procedure biotinylated oligonucleotides and the GCN-4 binding motif are incorporated into the PCR product during amplification. For detection of amplified CDNA, GCN-4 is coated to microtiter plates ( $75 \mathrm{ng} /$ well), the PCR reaction mixture overlaid, bound PCA products detected by binding a streptavidin-peroxidase conjugate to biotin, and subsequently quantified through a chromogenic reaction. A calibration curve was constructed using concentrations between 0.05 and $1.0 \mathrm{nmol}$ GPIb cDNA. Unspecific DNA binding was prevented with a mixture of albumine, milk powder and salmon sperm DNA. Formation of primeroligomers was excluded in control experiments. The low detection limit of 0.5 pmol CDNA as well as coefficients of intra- and interassay variations of $5.6 \%$ and $7.8 \%$, respectivelly, demonstrate that the colorimetric assay based on the GCN-4 DNA binding protein is a sensitive and specific assay which can easily be performed for quantitative determination of PCR products.

Hemostasis Research Unit, Kerckhoff-Klinik, Sprudelhof 11, D-61231 Bad Nauheim. 


\section{0}

A DELETION IN THE CYTOPLASMIC DOMAIN OF THE $\alpha$ SUNUNIT OF GPIb/IIIa $\left(\alpha_{m b} \beta_{3}\right)$ RESULTS IN AN IRREVERSIBEL ACTIVATED PLATELET-FIBRINOGEN-RECEPTOR

K. Peter, K. Weckesser, C. Bode

The integrin GP IIb/IIIa $\left(\alpha_{\mathrm{mb}} \beta_{3}\right)$ mediates platelet aggregation and adhesion by its binding of fibrinogen. For the adhesion molecule group of the integrins (more than 20 integrins are known until now) an inside out signaling is postulated during cell activation. Thus, signals from the cytoplasmic domains should be transfered to the extracellular parts of the integrin. We hypothesized, that such a common mechanism within the integrin group should be reflected in conserved amino acid sequences in the cytoplasmic domains. Using PCR we created a deletion of such a conserved region in the $\alpha$ subunit (GFFKR 1021-1025). After sequencing the cDNA construct was transfected $\mathrm{CHO}$ cells by electroporation. With G418-selection and FACS-sorting highly expressing cell lines were selected. FACS-analysis with an GP IIb/IIla activation specific mAb and binding studies with fibrinogen demonstrated an activated receptor. The metabolic inhibitors 2-deoxyglucose and $\mathrm{NaN}_{3}$ and truncations and point mutations in $B_{2}$ could not inhibit the activated state of the receptor.

Conclusions: A deletion of the GFFKR region in the cytoplasmic domain of the $\alpha$ subunit caused a signal transduction to the extracellular parts of GP Ilb/IIIa $\left(\alpha_{\mathrm{Ib}} \beta_{3}\right)$, resulting in an irreversible activated platelet-fibrinogen receptor. Deletion variants as this should provide a tool to dissect the role of affinity changes in cell adhesion, aggregation, and migration. Since the deleted region can be found in all integrin $\alpha$ subunits, similar activating effects may be expected in other integrins as for example the leukocyte adhesion molecules.

Innere Medizin III, Universität Heidelberg, Bergheimerstr. 58, 69115 Heidelberg

211

CHARACTERIZATION OF MUTATIONS IN THE FACTOR IX GENE

K. Wulff, M. Wehnert, W. Schroder and F.H. Herrmann

Haemophilia $B$ is an $X-1$ inked recessive bleeding disorder resulting from deficlency of factor IX proteine. The factor IX gene is mapped in the long arm of the $X-c h r o m o s o m e$ and spans $34 \mathrm{~kb}$. For the functional analysis of the factor IX gene mutations we analysed DNA from unrelated haemophilia B patients from different ethnic origins. A panel of 89 haemophilia B patients were screened of macroaberrations by Southern technique. No macroarrangements were found. Point mutations and microlesions were detected and characterized in the exon regions of factor IX gene by sequencing analysis. The results of the molecular analysis of factor IX gene are reported and discussed.
212

IN VIVO SOMATIC GENE TRANSEER WITH IKB AND TRUNCATED JUN SHOWS DEPENDENCE OF TNF MEDIATED INTRAVASCULAAR FIBRIN FORMATION ON NFKB AND AP-1

Y. Zhang, Y. Deng, Th. Luther, M. Müller, B.-S. Chen, R. Waldherr, R. Ziegler, P.P. Nawroth

Recently it has been shown, that induction of tissue factor (TF) by TNF in vitro is dependent on a concerted action of $N F k B$ and $A P-1$. However it remains unknown, if $T F$ can mediate intravascular fibrin formation and if $\mathrm{NFkB}$ and $\mathrm{AP}-1$ are involved in intravascular fibrin formation in vivo. When mice with Meth-A sarcomas were injected with TNF, TF was expressed by vascular endothelium of the tumor, fibrin deposited and free blood flow reduced. Intravenous somatic gene transfer with antisense-TF reduced $T F$, fibrin formation and restored free blood flow. THUS: TF is the mediator of TNF dependent fibrin formation in this in vivo model. Intravenous somatic gene transfer with IKB or truncated jun (still binding fos, but nor DNA) reduced $T F$ expression, fibrin formation and restored partially free blood flow. When IkB and truncated jun were injected together, the effect was more prominent.

In conclusion:

- Intravenous somatic gene transfer on the level of transcription factors is feasible and can be used to reduce $T F$ mediated fibrin formation in this animal model.

- TF is the initiator of fibrin formation in this in vivo model.

- TNF mediated TF induction is dependent on NFkB and AP- 1 .
213

A 381 Gly TO Ser CHANGE IN A PATIENT WITH A SYMPTOMATIC TYPE II PROTEIN C DEFICIENCY

E. Wittmann, J. Walter, B. Krinninger, I. Pabinger-Fasching, H.H. Watzke

Protein $\mathrm{C}$ is a vitamin K-dependent glykoprotein which plays an important role in blood coagulation. It circulates in plasma as a zymogen and is converted into its active form upon the interaction with thrombin. Activated protein $\mathrm{C}$ in turn inactivates the procoagulant cofactors $\mathrm{Va}$ and VIIIa. Protein $\mathrm{C}$ deficiency is inherited as an autosomal dominant trait and is associated with an increased risk of venous thromboembolism. The genetic basis of hereditary PC deficiency has been established in several kindreds by sequence analysis of the PC gene. We report the characterisation of the genetic defect in a family with PC-deficiency. The proposita of this family suffers from recurrent venous thrombosis $(V$. basilaris thrombosis, deep vein thrombosis). Her protein $\mathrm{C}$ activity is reduced to $58 \%$ of normal, her protein $\mathbf{C}$ antigen is within the normal range. All other inhibitors of coagulation were normal. Her sister has the same phenotype (PC activity $51 \%, \mathrm{PC}$ antigen $128 \%$ ), her brother has normal PC levels. In order to characterise the causative mutation of the proposita DNA was prepared from her white blood cells using standard methods. All 9 exons including the exon/intron junctions were amplified using the polymerase chain reaction. The PCR products were sequenced using dideoxy chain termination methode. A G to A transition of the nucleotide 8856 (according to Forster et al.) was detected which converts 381 Gly (GGT) to Ser (AGT) in the heavy chain of protein $C$. The proposita and her sister are heterozygous for the defect. The brother's PC gene is normal. Gly 381 is present in the corresponding position in all vitamin $\mathrm{K}$ depending proteins. It is in close proximity to the serin active center. The change from Gly to Ser at this position therefore propably affects the catalytic function of the molecule.

Dept. of Medicin I, Division of Hematology and Hemostaseology, Währinger Gürtel I8-20, A-1090- Wien
Present address: Institute of Medical Genetics University Greif swald, Germany

Fleischmannstr. 42/44, D-17487 Greifswald 
PROTEIN C INHIBITOR (PCD) IS EXPRESSED IN TUBULAR CELLS OF HUMAN KIDNEY.

Klaus-P. Radtke, José A. Fernández, Judith S. Greengard, Winson W. Tang, Curtis B. Wilson, and John H. Griffin.

Protein C Inhibitor (PCD), also known as PAI-3 is an inhibitor of the anticoagulant activated protein $\mathrm{C}$ and other serine proteases, among them urokinase ( $\mathrm{UPA}$ ) and urinary kallikrein. PCI has been identified as a component of human urine. However, the source of urinary PCI has not been previously identified. Since one possible source is kidney itself PCI mRNA and antigen were studied. PCI CDNAs were obtained from human and rhesus monkey kidney RNA. Restriction digestion of kidney PCI CDNA with endonucleases BclI or StyI resulted in band patterns identical to those obtained with liver PCI CDNA. Human and rhesus monkey kidney PCI cDNA were cloned and sequenced, and both showed identity with the sequence found for liver PCI cDNA from the respective species. Conditioned media from the rhesus monkey kidney cell line CCL7.1 was analyzed by SDS PAGE and immunoblotting, using monospecific antibodies against human plasma PCI. Kidney cell conditiuned media contained a $57,000 \mathrm{MW}^{\prime}$ protein band which comigrated with a control sample of purified human plasma PCI. Both bands reacted with antibodies against human PCI. Immunohistochemical staining of human kidney tissue sections using monospecific antibodies against human plasma $\mathrm{PCI}$ showed that the kidney PCI antigen was present and confined to tubular cells. Electron microscopy data showed $\mathrm{PCI}$ antigen within the ultrastructure of proximal tubular cells, confined to cytosomatic vesicles. These data provide evidence that $\mathrm{PCI}$ is expressed in kidney tissue. The findings have physiological significance for understanding the role of $\mathrm{PCl}$ in the kidney and urinary tract. The questions are raised whether uPA:PCI complexes found in urine may be locally produced in the kidney and whether PCI may be involved in the local control of u-PA fibrinolytic activity. The results suggest $\mathrm{PCI}$ may be involved in normal kidney physiology. Present address: University Clinic Frankfurt, Center of Internal Medicine, Frankfurt, FRG.
ANALYSIS OF SEMINAL PLASMA PROTEIN C INHIBITOR (PCI) IN NORMALS AND IN SELECTED PATIENTS

\section{X.L. Zheng, M. Geiger, and B.R. Binder}

Protein $\mathrm{C}$ inhibitor $(\mathrm{PCI})$ is a non-specific, heparin-binding serine protease inhibitor (serpin) originally described in plasma as an inhibitor of activated protein $\mathrm{C}$ and other coagulation factors. High concentrations of $\mathrm{PCI}$ are found in the male reproductive tract. We have shown recently that $\mathrm{PCI}$ inhibits acrosin and that endogenous $\mathrm{PCl}$ is present on damaged but not on intact sperm heads. Furthermore complexes of PCI with tissue plasminogen activator, urokinase and prostate specific antigen are found in seminal plasma. Therefore $\mathrm{PCl}$ might regulate several sperm- and seminal plasma proteases.

For further understanding whether $\mathrm{PCI}$ in seminal plasma might be related to diseases of the male reproductive tract, we analyzed $\mathrm{PCl}$ antigen in seminal plasma samples from normal, fertile men, from infertile patients (with normally and with poorly liquefying semen), and from patients with prostatitis. As judged from immunoblots using rabbit anti PCI-IgG and performed after SDS-PAGE, most PCI antigen in seminal plasma was either present in complexed or degraded form, supporting the involvement of $\mathrm{PCI}$ in the regulation of protease activity in the male reproductive tract. PCI antigen in individual seminal plasma samples was measured by an ELISA using acid treated monoclonal anti-PCI IgG $(10 \mu \mathrm{g} / \mathrm{ml})$ as a catching and peroxidase labelled rabbit anti-PCI IgG $(10 \mu \mathrm{g} / \mathrm{ml})$ as a detecting antibody. Seminal plasma PCI antigen level in childless men $(388.7 \pm 43.5 \mu \mathrm{g} / \mathrm{ml}$; means \pm SE), and in patients with prostatitis $(390.1 \pm 39.1 \mu \mathrm{g} / \mathrm{ml})$ were only slightly higher than in normals $(304.6 \pm 27.6 \mu \mathrm{g} / \mathrm{ml})$. A statistically significant difference was only found between patients with poorly liquefied semen $(476.5 \pm 43.7 \mu \mathrm{g} / \mathrm{ml})$ and normals $(\mathrm{P}<0.05)$, indicating that $\mathrm{PCI}$ might also regulate the activity of enzymes involved in semen liquefaction.

Clinical Experimental Physiology, University of Vienna, Schwarzspanierstr 17, A-1090 Vienna, Austria

\section{Varia I + II}

216

CALCIUM-DEPENDENT ACTIVATION OF PROTEIN C BY THROMBIN, THROMBOMODULIN: ROLE OF NEGATIVELY CHARGED AMINO ACIDS WITHIN THE ACTIVATION PEPTIDE OF PROTEIN C

Ute Friedrich, K. T. Preissner, G. Müller-Berghaus, H. Ehrlich, and B. Pötzsch

Activated protein $\mathrm{C}$ (PC) acts as an anticoagulant through proteolytic degradation of factors Va and VIIIa and has been used as an effective anticoagulant in the treatment of thrombotic diseases. Activation of $P C$ requires complex formation between thrombin and its cofactor thrombomodulin (TM). Thus, the concentration of endothelial cell expressed TM could become a critical factor for the uttimate therapeutic efficiency of substituted PC. In order to engineer a PC mutant which could be activated by thrombin independent of $T M$, attention was focussed to identify the molecular structures making PC-wild type to a poor substrate for free thrombin. Based on the hypothesis that such regulatory elements shouid be located near the thrombin cleavage site and should include negatively charged amino acids, we studied whether Asp and Glu in positions P6 and P7 relative to the thrombin cleavage site together with $A s p$ in $P 3$ are involved in $\mathrm{Ca}^{2+}$-dependent inhibition of $\mathrm{PC}$ activation by thrombin. $\mathrm{PC}$ mutants containing the neutral counterpart of the negatively charged amino acids in positions $P 3, P 3 / P 6$, and $P 3 / P 6 / P 7$, respectively, were generated using sitedirected mutagenesis and expressed in the eukaryotic cell line HU293. Compared to $\mathrm{PCC}$-wild type, the initial rates of activation of all PC mutants were increased 4.0-fold for activation by thrombin/TM and 4.0 - 5.3-fold for activation by thrombin alone. Compared to the $\mathrm{PC}$ mutant neutralized exclusively in P3, additional changes in P6 and P7 showed no increase in the thrombin activation kinetics. We conclude that 1 . among the three negatively charged amino acids within the activation peptide of $P C$, only Asp in P3 is involved in $\mathrm{Ca}^{2+}$ - dependent inhibition of PC activation by thrombin; 2. P7 and P6 sites are not required for $\mathrm{Ca}^{2+}$-dependent activation of $P C$ by the thrombin/TM complex.
217

The socioeconomic impact of substitution therapy in patients with hemophilia

Szucs TD, Rommel F, Schramm W

Background

Therapeutic interventions in patients with coagulation disorders, specifically in patients with hemophilia, have come into the crossfire of considerable criticism by health care reagulators and third party payers due to the high level of expenditure involved. Although the therapeutic benefit of modern substitution products has been well established, there is no comprehensive data available on the socioeconomic impact of therapeutic schemes in hemophiliacs. Such evidence is not only important for health care decision makers, but atso a basis for the future development of for health care decision mak
clinical treatment guidelines.

Rationale

Because aimost no data is available in Germany with respect to the economic benefits of prophylactic substitution, we have initiated a research program to systematically elaborate and compile socioeconomic data based on the extensive amount of clinical patient data available in the the Munich treatment center.

Material and methods

The baseline cohort in Munich consists of 17 patients receiving prophylactic treatment (PT) which are compared with 50 patients receiving on- demand therapy treatment
(ODT).

Results

The average amount of factor VIII substitution was $1927 \mathrm{U} / \mathrm{kg} / \mathrm{yr}$ with PT, and 833 $\mathrm{U} / \mathrm{kg} / \mathrm{yr}$ in the ODT group. These amounts represent average acquisition costs of DM 148000 (PT), resp. DM 68000 (ODT) per year and per patient. These costs are put into perspective with the economic impact of the clinical outcomes in the two groups. The average number of joint bleeds per year was 3 (PT), resp. 16.3 (ODT), the number of days off work per year was 3 (PT), resp. 10.1 (ODT, excluding patients $<20 \mathrm{yrs}$ ). These clinical outcomes have been valued in economically by estimating the average amount of direct and indirect medical resources consumed per year. In PT patients we estimate the economic impact per patient per year DM 4100, in ODT patients DM 8000 , without valuing the negative impact on the qualiy of life in those patients experiencing bleeds and pain. The average costs per avoided bleed using PT is approximately DM 5700 .

Discussion

Although the overall financial investment in the prophylactic treatment seems to be Although the overall financial investment in the prophylactic treatment seems to be
much higher than the on-demand strategy, it should be discussed whether the much higher than the on-demand strategy, it should be discussed whether the
additional costs of DM 5700 to avoid one joint bleed is worthwhile from a policy point additional costs of DM 5700 to avoid one joint bleed is worthwhile from a policy point of view. These data are only preliminary results of a small cohort. More research will
be conducted by the Munich center in the near future, with the objective of elucidating the socioeconomic benefits of Pr. 
218

The Cost-Effectiveness of Ticlopidine in Secondary Prevention of Stroke

\section{Angela Kempel, PHARMAMETRICS GmbH, Freiburg}

The risk of secondary stroke is generally approximately $28-38 \%$ (based on literature review), whilst risk is highest in the first year after the initial attack, and the overall prognosis for TIA progressing to stroke is $32-51 \%$. According to the TASS Study (Hass, 1989) the risk reduction for fatal and non-fatal stroke under treatment with Ticlopidine was $46.2 \%$ after the first year, and the overall risk reduction after three years was $21.3 \%$ in comparison to Aspirin. Thus, allocation to treatment with Ticlopidine for 100 patients has the potential of avoiding a significant number of recurrent events (i.e. strokes) in those with prior TIA or stroke compared to nontreatment. Also, allocation to treatment with Ticlopidine can avoid 3-4 strokes more per 100 patients treated than treatment with Aspirin.

Cost-effectiveness of Ticlopidine in the avoidance of stroke has therefore been assessed (retrospectively) on the basis of the results from the TASS Study. Effectiveness of therapy (Ticlopidine vs. Aspirin) was measured as the number of strokes avoided. Costs of therapy included the cost of drugs, monitoring test recommended by manufacturers, complications of therapy (side effects) and cost of events. Finally, allocation to treatment with Ticlopidine may result in an overall monetary benefit of DM 91,681.- (in Germany) per 100 patients treated considered to be at high risk of vascular events (prior TIA or stroke). Thus, overall, the allocation to treatment with Ticlopidine shows to be cost-effective in comparison to Aspirin even in spite of the substantially higher treatment cost of Ticlopidine. Whilst direct cost for monitoring, complications of therapy showed no significant difference in the two groups of patients, the significant difference of cost is mainly based on cost of events. The range of average cost per event lies between a minimum of DM 27,000.- for patients totally recovered after the attack but who were not in the workforce and a moximum of $D M 118,711$.- for the first year after the attack for patients left handicapped and who have been in the workforce before the event.

219

HEMORHEOLOGICAL PARAMETERS IN DIABETIC PATIENTS AFTER SIMULTANEOUS PANCREAS-KIDNEY TRANSPLANTATION

Armin J. Reininger!, Achim C. Mellinghoff ${ }^{2}$, Rüdiger Landgraf ${ }^{2}$, Laurenz J. Wurzinger 1

Diabetes mellitus is a disease often associated with impaired macro- and microcirculation as well as disturbed hemorheology. Thus we compared established and hypothesized risk factors with blood and plasma viscosity in a group of diabetic patients following simultaneous pancreas-kidney transplantation.

The 64 patients ( 34 female, 30 male) aged $39.7 \pm 7.5$ years had a mean diabetes duration of $28.4 \pm 6.4$ years and a mean interval of $4.5 \pm 2.8$ years since transplantation. 46 patients exhibited arterial hypertension and 24 patients had macroangiopathy. The patients were divided into two groups: In group I $(n=29)$ both transplanted organs were functionally intact; in group II $(n=35)$ only the renal graft functioned and insulin dependency was present $(n=32)$. In the latter group, $\mathrm{HbA}_{1 \mathrm{c}}$ was significantly increased $(\mathrm{p}<0.001$ ). No stastistically significant differences were found for: age, diabetes duration, body mass index, blood pressure, total protein, albumin, $\alpha_{2}$-globulines, fibrinogen, triglycerides, cholesterol, LDL-cholesterol, HDL-cholesterol and creatinine. However, kinematic as well as dynamic viscosity of plasma and whole blood (shear rates ranging from $10 \mathrm{~s}^{-1}$ to $100 \mathrm{~s}^{-1}$ ) were significantly increased in group II $(\mathrm{p}<0.05)$. Moneover, linear regression analysis demonstrated a correlation between daily insulin dose and kinematic as well as dynamic viscosity for shear rates ranging from $100 \mathrm{~s}^{-1}$ to $600 \mathrm{~s}^{-1}(\mathrm{p}<0.01, \mathrm{r}=0.5)$.

Our findings suggest that hemorheology in diabetic patients is positively influenced by successful pancreas-kidney transplantation. Conversely hyperinsulinemia, necessary for optimal metabolic control in the renal graft recipients without functioning pancreas, seems to increase blood and plasma viscosity independent of plasma protein concentrations.

1 Anatomisches Institut, Technische Universität München, Biedersteinerstr. 29, 80802 München, Germany

${ }^{2}$ Medizinische Klinik Innenstadt, Ludwig-Maximilians-Universität

München, Ziemssenstr. 1, 80336 München

\section{0}

STUDY OF CORRELATION BETWEEN VON WILLEBRAND FACTOR PLASMATIC LEVEL AND THE ACTIVATOR INDUCED PLASMA CLOT LYSIS TIME IN PATIENTS WITH ADVANCED LIVER CIRRHOSIS

K.Rak, E.Posan, I.Tornai and M. Udvardy

A sustained high level of von Willebrand factor (vWF) is consequently found in plasma of patients suffering from Laennec cirrhosis. Although there are no specific abnormalities, different observations indicate that enhanced fibrinolysis and a certain degree of intravascular coagulation are commonly present. A pharmacologically induced or a pathological fibrinolytic state (mainly the degradation products) may be important in stimulating synthesis and release of vWF from the storage pool, first from the endothelial cells. In order to study a possible causal relation between vWF level and fibrinolytic potential, 40 patients with advanced liver cirrhosis were investigated. Methods vWF plasmatic level measured by ELISA using home produced monoclonal antibody. Lysis of platelet poor plasma (PPP) induced by thrombin was initiated by streptokinase or tissueplasminogen activator and the lysis time and speed was measured. Vmax (a kinetical parameter) was obtained, its value is directly proportional to the clot lysis. Results: clot lysis (Vmax value) was in an inverse correlation with vWF plasmatic level(linear correlation coefficient: -0.458 ). High vWF level (over $2.0 \mathrm{U} / \mathrm{ml}$ ) was accompanied mostly by a decreased fibrinolytic activity. Conclusion: our findings do not support an important contribution of enhanced fibrinolysis to the elevated plasmatic level of $\mathrm{VWF}$ in cirrhotic patients.

Present address: IInd Department of Medicine, University Medical School H-4012 Debrecen, Hungary

221

CLINICAL AND LABORATORY INVESTIGATION OF PATIENTS WITH CEREBRAL ISCHEMIA UNDER PENTOSAN POLYSULFATE THERAPY Imre G. Szirmai $\$$, Maria E. Scholz\$\$, Anita Kamondi $\$$ Department of Neurology, Semmelweis University Budapest, Hungary, bene, Pharmaceutical Company, Münich, Germany

I. Acute effect of pentosan polysulfate (PPS) an orally applicable heparinoid: Forty three patients with ischemic stroke received 4 $\mathrm{mg} / \mathrm{kg}$ PPS in one hour period, i.v. Euglobulin lysis and Quicktime decreased significantly, prolongation of partial thromboplastin time (APTT) and thrombin time lasted for $2-4$ hours. Anticoagulant effect of PPS related inversely to the level of high density lipoprotein (HDL).

II. Follow up investigation: Seventy four patients with ischemic stroke received PPS continuously (200-400 mg/ day) orally over $2-6$ years (mean $=4.2$ years). Control patients received no anticoagulants during the observation period. In the treatment group significant decrease of initial hematocrit and increase of APTT was observed; however plasma fibrinogen, cholesterol and $\mathrm{HDL}$ level remained uneffected. The neurological condition of patients improved significantly in both groups after the acute stage of disease. Frequency of recurrent stroke and death in the control group was higher than in the treatment group by $32 \%$ and $25 \%$ respectively.

Conclusion: I.v. administered PPS proved to have strong fibrinolytic and heparin-like effect on the blood coagulation. Oral medication reduced the frequency of recurrent stroke and improved the life expectancy of patients. 


\section{EXPERIMENTAL AND COMPUTATIONAL ANALYSIS OF THROMRUS FORMATION ON INDWELLING CATHETERS Peter Friedrich and Armin J. Reininger $\#$}

Thrombotic occlusion is a major complication affecting indwelling catheters. Besides a foreign surface effect, disturbances in blood flow are thought to be causative. Thus, flow disturbances encountered at catheter tips were visualized, quantitatively assessed as well as computationally simulated and subsequently compared to in vitro thrombus growth. The complex in vivo geometry was simplified to a central inner tube and a straight outer tube. The latter was perfused with whole blood, plasma or fibrinogen solution. At the distal end of the catheter an annular vortex was present. Induced coagulation resulted in thrombus formation at the catheter tip. Thrombus morphology and localization were similar for all perfusion fluids. The thrombus grew from the distal end of the catheter into the interface between bulk flow and the annular vortex to finally fill the entire vortex region. In order to identify the flow parameters responsible for thrombus localization, calculation of flow velocity, shear rate, streamline distribution and residence time was performed for three different stages of thrombus growth derived from the flow experiments. Regions of thrombus formation always correlated with regions exhibiting the longest residence time.

In a second set of computational modeling, residence time was used as the only variable to simulate thrombus growth. The increase in viscosity caused by fibrin polymerization was introduced into the computation. Thrombus growth was indicated as the region of maximum viscosity. The computation rendered thrombi identical in size, form and localization to those obtained experimentally. Furthermore, not only final thrombus morphology but intermediate growth steps as well exhibited close similarity.

Our results demonstrate that residence time distribution is the only variable required for computational simulation of fibrin thrombus formation in regions of disturbed flow at the tip of indwelling catheters.

GSF-Forschungszentrum für Umwelt und Gesundheit, Abteilung für Physiologie, Ingolstädter Landstr. 1, 85758 Oberschleißheim, Germany; \# Anatomisches Institut, Technische Universität München, Biedersteiner Str. 29, 80802, München, Germany

\section{COLLAGEN-TARGETED ANTIBODIES INHIBIT PLATELET- DEPENDENT THROMBOSIS}

\section{G.V. Bashkov, I.P. Stepanova and S.P. Domogatsky}

\begin{abstract}
Subendothelial collagen is one of the main triggers of platelet-
\end{abstract} dependent thrombus formation in arteries. The antithrombotic action of rabbit polyclonal inhibitory antibodies to rat collagen type I-III, murine non-inhibitory monoclonals to human urinary twochain/one-chain urokinase-type plasminogen activator (tcu/scuPA), cross reacting with rat urinary tou/scu-PA and their chemically synthetized conjugate was studied both in vitro and in vivo. Treatment of rat collagen immobilized with the polystirol surface by the anticollagen antibodies or bifunctional conjugate markedly inhibited human platelets adhesion and formation of platelet-rich thrombi-like structures in a conditions mimics the blood flow in large arteries. Monoconals to human tcu/scu-PA did not inluence on the collagen-induced platelet activation in vitro. The short-term treatment of collagen-soaked silk thread by anticollagen antibodies suppressed the platelet-dependent thrombus formation by $56 \pm 4 \%$ $(P<0.001)$ in the rat arteriovenous shunt thrombosis model. Bispecific conjugate directed both to rat collagen and tcu/scu-PA inhibited thrombus formation in the same magnitude as the anticollagen antibodies (by $44 \pm 4 \%, P<0.001$ ). The treatment of collagen-adsorbed conjugate by recombinant human tcu-PA did not increase the antithrombotic effect of bifunctional antibodies. The present data suggest that the local administration of the anticollagen antibodies to the site of vascular injury may be the efficient tool for the prevention of platelet-dependent thrombus formation in arteries after thrombolysis or percutaneous transluminal angioplasty.

Lab. Blood Coagulation, National Haematological Scientific Center, Novo-Zykovski pr. 4A, Moscow 125167, Russia.
NO EVIDENCE FOR REBOUND ACTIVATION OF PLATELETS AND COAGULATION AFTER LONG-TERM ADMINISTRATION OF PEG-HIRUDIN (LU 57291) IN RATS

W. Hornberger, M. Kluge, K. Rübsamen

The long-term use of thrombin inhibitors for the antithrombotic therapy bears the potential of thromboembolic complications due to resurned or fortified activation of platelets and the coagulation system after cessation of treatment. it is currently being discussed whether this phenomenon is due to an actual rebound mechanism (mediated e.g. by upregulation of thrombin receptors or downregulation of plasmatic thrombin inhibitors) or a continuation of the underlying procoagulatory mechanisms (Willerson \& Casscells, J Am Coll Cardiol 21, 1048-51, 1993). From a theoretical point of view the risk of thromboembolic events after cessation of treatment seems even higher for compounds that are rapidly cleared from the circulation like argatroban or hirulogs than for tight-binding inhibitors with sustained duration of action like polyethyleneglycol-coupled hirudin (PEG-hindin).

The aim of this study was to determine the efficacy of PEG-hirudin on thrombus formation. blood coagulation, platelet function and thrombin generation capacity in rats after cessation of a long-term treatment with PEG-hinudin.

PEG-hirudin or placebo were administered to rats for four days either as an intravenous bolus injection of $1 \mathrm{mg} \cdot \mathrm{kg}^{-t}$ once daily or by continuous intravenous infusion of $0.215 \mathrm{mg}^{\cdot} \mathrm{kg}^{-1} \cdot \mathrm{h}^{-1}$ using osmotic minipumps. 24 hours later, after the compound had completely been excreted, the antithrombotic effect of PEG-hirudin compared to placebo was evaluated in an arteriovenous shunt model $5 \mathrm{~min}$ after intravenous bolus administration of $0.316 \mathrm{mg}^{-} \mathrm{kg}^{-1}$. APTT, anti-Flla activity in plasma, thrombin-induced platelet aggregation and thrombin generation capacity in platelet-rich and plateletpoor plasma were determined ex vivo on day 1,2 and 4 after cessation of treatment. The antithrombotic effect of PEG-hirudin one day after cessation of long-term treatment was similar in both groups. There was no difference in APTT, anti-Flla activity and platelet aggregation induced by 3.16 or $4.64 \mathrm{U} / \mathrm{ml}$ thrombin. Thrombin generation capacity compared to the placebo-pretreated group one, two and four days after cessation of treatment was identical after extrinsic or intrinsic pathway stimulation in both platelet-rich and platelet-poor plasma systerns.

These data support the assumption that long-term administration of PEG-hirudin is not followed by rebound phenomena caused by enthanced thrombin formation or thrombin sensitivity.

Knoll AG, Dept. of Cardiovascular Pharmacology, D-67008 Ludwigshafen
LOOK-OUT FOR PREDTCTIVE UARUERS FOR PREMATURE RECOGNATION OF CORPLICATIONS IN PAIIENTS WITH LEUCEHIA

H. Scheel, A. Siegemund, W. Helbig, I. Polednia

Complication in conditioning phase and after transplantation of bone-marrow have a negatively effect on the prognosis in patients with leucemia. For that reason we have investigated molecular markers for therapeutic and prophylactic arrangements. In a pilot study patients with AIL and CII, with allogenic respectively autologous bone-narrow transplantation, as well as patients with peripheral stem cell transfusion by lymphogranulonatosis, IEG-plasmocytoma and rhabdomyosarcoma were investigated.

Following prognostic markers were measured: the activation markers of coasulation and fibrinolytic systems, such as the markers of endothelial cell injury ( $t-P A, P A I$, TH and THE). The blood samples were taken in an fixed time regime for and after transplantation. For suppressing the activation of coagulation and for thrombosis prophylaxis all of patient were given heparin; deficiences in haemostasis potential were substituted with blood factors and thrombocytes.

In our investigation we found, that in the conditioning phase both the quotient of $\operatorname{PAI}(1): \mathrm{Ag}$ to $t-\mathrm{PA}: \mathrm{Ag}$ and the circulation thrombomodulin is increased. A new increase of this quotient and o thrombomodulin after bone-marrow transplantation has an unfavourable influence; iminent complications such as GVHD and VOD are possibly axxociated with it.

Centre of Internal Medicine

University of Leipzig, Germany 
MECHANISM OF DEFIBROTIDE ON HAEMOSTASIS

J. Junker, J. Storck, and R.E. Zimmermann

Defibrotide, a DNA derivative of mammalian origin showed antithrombotic and profibrinolytic effects in vivo. Despite numerous approaches, the molecular basis of action is not well understood. The present study was undertaken to find the mechanism of defibrotide on haemostasis by testing its activity to some key components of haemostasis: fibrinogen, plasminogen, tissue plasminogen activator, and cathepsin G. The binding behaviour between defibrotide and these proteins was tested by affinity chromatography. Defibrotide was coupled to cyanbromide activated sepharose to yield defibrotide sepharose. Protein samples were applied to the defibrotide sepharose column and then eluted by a linear gradient from $0 \mathrm{M}$ to $1 \mathrm{M} \mathrm{NaCl}$. Plasminogen was eluted at $0.15 \mathrm{M} \mathrm{NaCl}$, fibrinogen at $0.25 \mathrm{M} \mathrm{NaCl}$, tissue plasminogen activator at $0.3 \mathrm{M} \mathrm{NaCl}$, and cathepsin $\mathrm{G}$ at $0.7 \mathrm{M} \mathrm{NaCl}$. These results demonstrate, that the tested proteins bind at physiological salt concentrations to defibrotide. In the case of defibrotide bound tissue plasminogen activator the plasminogen activation activity was preserved. This implies, that defibrotide could function as artificial surrogate for fibrin mediated plasminogen activation by tissue plasminogen activator. The observed cathepsin $G$ affinity correlates well with the antiplatelet activity of defibrotide described by Evangelista et al. (Thromb. Haemost. 67, 660, 1992).

Universität Münster, Institut für vegetative Physiologie, Robert-Koch-Straße 28,48149 Münster

\section{7}

EXPERIMENTAL THROMBOSIS OF THE ARTERIA RENALIS G. Nowak, E. Bucha, S. Basche ${ }^{\star}$

Arterial thrombosis in animal experiments can be effected in several ways: by placing a copper coil in a vessel, injuring the vessel wall by direct current, removing the endothelium mechanically, or by administering aggressive chemical substances into the inside (e. g., organic acids, alcohols) or onto the outside (e. g., silver nitrate) of vessels. The patterns of endothelial damage thus obtained are coarse, being in no way consistent with pathophysiological courses of reaction involved in thrombogenic change of endothelium. For this reason, new ways have been seeked for the induction of thrombosis approaching pathophysiological reactions. Using radiopaque microcatheters and an $X$-ray visualization system, the $A$. renalis dextra of anaesthetised rabbits was represented. A hypertonic glucose solution $(10 \%)$ provided with $30 \%$ radiopaque medium and heated up to $70^{\circ} \mathrm{C}$ was administered via catheter at a dose of $2 \mathrm{ml} / \mathrm{kg}$ body weight, using high pressure. The endothelium peripheral to the tip of catheter was thermaliy damaged by short-term displacement of the blood flow. The right kidney was found to be completely embolised within 10 to 15 minutes; total thrombosis of the A. renalis could be detected about 10 minutes later. In controlling the blood flow, using a solution of diluted radiopaque medium, total displacement of the right kidney's blood supply was detectable. This method is suitable for producing within a relatively short period: a) localised microthrombosis/embolisation of microvasculation of organs or body regions and $b$ ) arterial thrombosis due to localised endothelial damage.

Max-Planck-Gesellschaft e. V., Research Unit for Pharmacological Hemostaseology at the University of Jena, Drackendorfer Str. 1, D-07747 Jena, FRG

*Department of Diagnostic Radiology, Medical School Erfurt, Nordhäuser Str. 74, D-99089 Erfurt, FRG

\section{8}

IONIC INTERACTIONS BETWEEN LAMBDA-CARRAGEENAN AND COAGULATION FACTORS

U. Lange, G. Nowak

Carrageenans are high molecular, sulfated polysaccharides of plant origin which are extracted from red algae. The chemical backbone of carrageenans consists of alternately linked $\beta(1 \rightarrow 4)$ and $\alpha(1 \rightarrow 3) D$-galactose molecules. When administered parenterally, carrageenans produce a variety of biological effects in laboratory animals. Carrageenans cause both thrombogenic and anticoagulant effects on the coagulation system. In the literature, interactions with plasma proteins, especially proteins of the coagulation system and their precipitation due to carrageenans, have been described and have, among other things, been cited to explain the anticoagulant effects of carrageenans. We studied in vitro interactions of lambdacarrageenan - a type of carrageenan characterized by a high amount of sulfate residues - with the coagulation proteins thrombin and fibrinogen. We investigated the influence of lambda-carrageenan on the amidolytic and clotting activities of thrombin in the presence and absence of antithrombin and heparin colactor II. To this end, we performed a clotting assay using fibrinogen as substrate and an amidolytic assay employing $\mathrm{N}$-p-tosyl-Gly-Pro-Arg-p-nitroanilide as chromogenic substrate. Both in the amidolytic and the clotting assay lambda-carrageenan was found to inhibit the activity of thrombin directly. In the presence of antithrombin and heparin cofactor II, resp., this direct thrombin inhibition was intensified only moderately. An increase in ionic strength diminished, or even neutralized, the direct antithrombin activity of lambda-carrageenan in the amidolytic assay, suggesting ionic interactions between thrombin and lambda-carrageenan. Investigating the interactions between lambda-carrageenan and fibrinogen, we could demonstrate that lambda-carrageenan precipitates fibrinogen from solutions, depending on the concentration used. In the clotting assay, the precipitation of fibrinogen was found to play only a minor role in prolongation of the ciotting time. Also the interactions between lambda-carrageenan and fibrinogen depend on the ionic composition of the solution: $\mathrm{Ca}^{++}$ions intensified the precipitation of fibrinogen by lambdacarrageenan, while $\mathrm{Na}^{+}$ions inhibited these $\mathrm{Ca}^{++}$effects on fibrinogen precipitation. The present results suggest that ionic interactions between lambdacarrageenan and coagulation factors, especially thrombin and fibrinogen, may play a role in lambda-carrageenan's effects on the coagulation system in vivo.

Max-Planck-Gesellschaft e. V., Research Unit for Pharmacological Hemostaseology at the University of Jena, Drackendorfer Str. 1, D-07747 Jena, FRG
229

ACQUIRED FACTOR XIII DEFICIENCY WN A PATENT WTTH STAPHYLOCCOCAL SCALED SKTN SYNDROM

M Nader, N. Endres, U. Schweigart, R. Lorenz, G. Mößmer, M. Classen

Acquired deficiency of factor XIII has yet been reported in disseminated intrapascular coagulation, severe liver deseases with impaired synthetic function, chronic inflammatory bowel diseases, akute leukemia, Henoch Schoenlein purpura, in the postoperative phase and in severe burns.

We report the case of an 36 year old male who developed, cutgoing from a superinfected face injury, a staphyloccocal scaled skin syndrom- involving about $30 \%$ of total skin surface At admission the patient showed tachycardia up to 140/min, hypotension with a need for catecholamines and insufficient breathing Fever was maximal $39^{\circ} \mathrm{C}$, there was a pleural empyema and highly suspicion of mediastinitis. In wound-swabs, urin and blood enterocooci and staphylococci were found. There was initial leukopenia $(3.1 \mathrm{G} / 1)$ but leukocytosis ( $27 \mathrm{G} /$ ) after 72 hours. During the observation period there was no significant elevation of liver enzymes (bilirubin max. $2 \mathrm{mg} / \mathrm{d}$ ) and no signs of renal failure or disseminated intravascular coagulation. But with the appearence of tallow skin lesions a marked reduction of factor XIII plasma activity was observed (plasme level 11\%, normal teve1 75-100\%). Repeaded substitiution of factor XII concentrate (Fibrogammin HS, Behring. Mannheim) led only to a transient elevation of factor XIII levels (max 54\%) within a period of 4 weeks but normalized spontaneously after healing of the skin lesions. The plasma levels of factors II VII and AT III were decreased in the beginning of the disease but recovered spontaneously within a few days.

In our opinion there is a significant loss ore increased consumption of factor XIII via bullous skin lesions in analogy to the already known factor XII losses in severe burns. Factor XIII substitution mey be a beneficial therapeutic approach in that cases like- as already shown -in impaired wound healing and severe burns.

II Med. Klinik und Poliklinik, Institut fur Klinische Chemie und Pathobiochemie der Technische Universităt München, Ismaningerstr. 22, 81675 München 
INELUENCE OF ENDOSCOPIC MANIPULATIONS IN THE BILIARY TRACT ON THE COAGULATION SYSTEM

R. Lorenz, M. Herrmann, N. Iehn, and P. Born

Obstructions of the bile ducts require drainage procedures by endoscopic retrograde cholangiography (ERC) or percutaneous transhepatic cholangiography (PTC). In 2-88 of these cases septical. complications occur. Because of the unknown influence of these diagnostic and therapeutic procedures on the hemostatic system, we carried out a prospective study including 30 patients suffering from biliary obstruction.

In all patients, endoscopic drainage by means of ERC or PTC inclusive papllotomy, lithotripsy or implantation of biliary stents were performed. Neither laboratory nor clinical signs of septicenia could be detected before endoscopy. Before endoscopy as well as 2 hours, on day 1 and day 2-4 after endoscopy samples of citrated blood were taken and the following paras meters were determined: platelet count, fibrinogen, thrombinantithrombin-complexes (TAT), plasminogen, $\alpha$-plasmin-inhibitor $\left(\alpha_{2} \mathrm{AP}\right)$, prekallicrein (PK) and antithrombin III-activity. The preendoscopic value of TAT was raised $(6.2+3.8 \mathrm{ug} / 1)$, whereas the iritial values of the other parameters were in their physiological ranges. The TAT concentrations showed a significant increase 2 hours after endoscopy $(36.4+29.2 \mathrm{ug} / 1 ; \mathrm{p} 0.01$ and after day $1(21.2+29 \mathrm{ug} / 1 ; \mathrm{p}<0.01)$ compared to the initial values. There was a significant drop of PK $(79.7+20.6$ vs. 85.8 $+228 ; \mathrm{p}<0.05), \alpha$ AP $(87.2+11.2$ vs. $90.6+13.18 ; \vec{p}<0.05)$, fibrinogen $\left(372+126^{2} \mathrm{vs} .407+\overline{1} 23 \mathrm{mg} / \mathrm{dl} ; \mathrm{p}<\overline{0} .05\right)$ and the platelet count $(25 \overline{8}+70$ vs. $271+76 \mathrm{G} / 1)$ after 2 hours, each compared to the initial values. Plasminogen and AT III activity remained unchanged.

In conclusion, these changes can be interpreted as an activation of hemostasis leading to the risk of both a prethrombotic state and a beginning intravasal fibrin generation. The clinical consequence should be a wide indication for thromboprophylaxis in cases with invasive endoscopic procedures in the biliary tract.

II. Medizinische Klinik, TU München, Klinikum r.d.Isar, Ismaninger Straße 22, 81675 München

\section{1}

\section{PREDICTABILTTY OF BLOOD COAGULATION PARAMETERS IN PATIENTS WITH MULTIPLE ORGAN FAILURE \\ D. Mentzer, L. v.Beck*, W. Kreuz, H.K. Breddin*, G. Klein**, C.M. Kirchmaier*}

Disseminated intravascular coagulation (DIC), a major complication in intensive care patients, often leads to multiple organ failure (MOF). It is based on different pathological events (e.g. ARDS, renal failure).

Therefore we initiated a study to find out, whether the disturbances of the blood coagulation system in patients with DIC correlate with MOF-Score according to Marschall et al. (1988).

Different clinical and blood coagulation parameters (e.g. Reptilase-, Thrombincoagulasetime, AT III and HC II activity) of 43 patients with septicemia were investigated during follow-up in our intensive care unit

Patients with DIC $(n=33)$ were divided into groups with and without substitution of AT III concentrate. In the group of patients with DIC, 21 patients were substitutied with AT III concentrate, 13 of these patients showed initial AT III activity $<50 \%$ at onset of AT III replacement. 12 out of 33 patients with DIC were not substituted with AT III concentrate during follow-up.

Patients without DIC $(\mathrm{n}=10)$ did not receive any AT III replacement and none of these patients died during follow-up.

Blood coagulation parameters (Reptilase-, Thrombincoagulasetime, AT III and HC II activity) were positively correlated with MOF-score.

Patients with DIC and AT III replacement had better prognosis compared to the group without AT III substitution. Patients with DIC and AT III replacement showed a significantly positive improvement in the MOFscore during follow-up dependant to initial AT III activity.

In the group without AT III replacement no positive progression of the MOF-score was found.

Increased MOF-score correlated significantly with increased fibrinolysis, loss of AT III and HC II activity in patients with septicemia, independant to acquired DIC with or without AT III replacement. Clinical outcome of patients with DIC depended on progression of MOF-score and was influenced by duration and onset of AT III treatment.

\section{Haemostaseological risk indicators in progress of a deep venous thrombosis and their relationship to the inflammatory reaction (CRP)}

O. Anders', H. Auelz, C. Burstein', H. Ehrensberger ${ }^{3}, N$. Hegner ${ }^{4}, V$. Hossmann ${ }^{2}$ F. Keller ${ }^{3}$, H. Kujawska $a^{5}$. H. Lind ${ }^{4}$, W. Müller-Beißenhirtz ${ }^{6}$, G. Müller-Berghaus H. Scheel, A. Siegemund ${ }^{8}$, U. Taborski? ${ }^{7}$ N. Weinstock ${ }^{9}$

In a multicenter study amòngst 8 dinics haemostatic risk indicators and CRP were measured in patients with deep venous thrombosis during hospital stay $(n=73)$ and at an ambulatory follow-up examination $(n=63)$.

At the time of admission the following percentages of increased or decreased values of the examined risk indicators were found:

Fibrinogen $\uparrow: 82 \%$, Ristocetin-Cofactor $\uparrow: 43 \%$, Protein $\$ \downarrow: 36 \%$, PAl $\uparrow: 22 \%$, Protein $C \downarrow: 10 \%$, Plasminogen $\downarrow: 8 \%$, Antithrombin $111 \downarrow: 4 \%$.

At this time $90 \%$ of the examined patients had increased CRP values.

At the ambulatory follow-up examination $66 \%$ of the patients had normal CRP values again, $34 \%$ of the patients had moderately increased CRP concentrations.

In the group with normal CRP the percentages of patients with values above or below the normal range were as follows:

Fibrinogen $\uparrow: 49 \%$, PAl $\uparrow: 35 \%$, Ristocetin-Cofactor $\uparrow: 24 \%$, Plasminogen $\downarrow: 14 \%$, Antithrombin III $\downarrow: 5 \%$, Protein $S \downarrow: 4 \%$, Protein $C \downarrow: 4 \%$.

Patients with increased CRP values had about the same incidence of pathological values of fibrinogen, ristocetin-cofactor and PAI values as at the admission.

During the time free of complaints the values found often increased were fibrinogen, von Willebrand factor (as ristocetin-cofactor-activity) and PAI. In prospective studies these parameters were shown to correlate with arterial thrombosis. This leads to the assumption, that correlation between arterial and venous thrombosis is greater than expected in the past. Fibrinogen and ristocetin-cofactor are highly related to thrombocyteaggregation and -adhesion.

I Universitätsklinik Rostock

${ }^{3}$ Universitätsklinik Würzburg

${ }^{5}$ Bezirksksankenhaus Halle

' Kerckhoff-Klinik Bad Nauheim

${ }^{9}$ Städtisches Klinikum Karlsruhe

\author{
${ }^{2}$ Krankenhaus Köln-Porz \\ 4 Behringwerke AG Liederbach \\ ${ }^{6}$ Bürgerhospital Stuttgart
}

${ }^{8}$ Universitätsklinik Leipzig

\section{CORRELATION BETWEEN ECLT, T-PA AND PAI-1 ACTIVITY IN HEALTHY VOLUNTEERS \\ M.Dávid, H. Losonczy}

Our aim was to study the correlation between euglobulin clot lysis time (ECLT), t-PA activity and PAI-1 activity in healthy volunteers. 27 volunteers (17 males, 10 females) were examined. Their mean age was $24.89 \pm 4.46$ years (range 19-37 year).

To investigate the fibrinolytic system ECLT, t-PA activity, PAI-1 activity, t-PA and PAI- 1 antigen, plasminogen, $\alpha_{2}$ antiplas$\mathrm{min}$, fibrinogen and D-dimer was performed (the first three before and after venous occlusion test). Strong negative correlation was found between ECLT and $t-P A$ activity before venous occlusion test and weak correlation between ECLT and PAI-1 activity before venous occlusion test.

According to ECLT after venous occlusion test 2 groups of volunteers could be formed. Poor responders where the ECLT $>100$ minutes, gond responders where ECLT $<100$ mintites aftiti venous occlusion test. Significant differences could be found between the good and poor responder groups in the ECLT and $t-P A$ activity, but there was no difference between the 2 groups' results in PAI- 1 activity. In the poor responder group, however, elevated PAI-1 antigen level was found.

According to our results the ECLT represents the extrinsic fibrinolysis, mostly the t-PA activity. In controlling the t-PA activity the PAI-1 antigen level has an important role in the formation of the $t-P A / P A I-1$ complex.

1st Department of Internal Medicine Medical School of Pécs 7624, Pécs, Ifjuság u. 13. Hungary 
THE SYNERGISM OF THROMBOLYTIC ACTION BETWEEN STREPTOKINASE (SK) AND SINGLE-CHAIN UROKINASE-TYPE PLASMINOGEN ACTIVATOR (Scu-PA) IN A PLASMA MILIEU IN VITRO

G. V. Bashkov, Yu. V. Jitkova, V. A. Makarov

The thrombolytic effects of SK, scu-PA and their combinations were measured in an in vitro system composed of a $125_{1-f i b r i n}$ labelled human plasma clot immersed in a citrated human plasma at $25^{\circ} \mathrm{C}$. Clot lysis was monitored by $125_{1-\text { fibrin degradation }}$ products release and activation of the fibrinolytic system in the surrounding plasma was measured by the fibrinogen, plasminogen and $\alpha_{2}$-antiplasmin level. SK and scu-PA induced a dose- and timedependent clot lysis: 50 percent lysis after $4 \mathrm{~h}$ was obtained with 70 $\mathrm{nM}$ SK and $50 \mathrm{nM}$ scu-PA. At these concentrations no significant activation of the fibrinolytic system in plasma was observed with scu-PA, whereas SK produced the marked depletion of the fibrinolytic system and fibrinogen breakdown. As was judged by the mathematical analysis of dose-response curves by the isobole and original analytical methods the combinations of SK and Scu-PA in a molar ratios of $1: 3$ to $3: 1$ caused the synergistic thrombolytic effect, exepting cases when SK and scu-PA concentrations were less than $10 \mathrm{nM}$. The maximal achievable 2.5 -fold increase in the thrombolytic action of this combination was observed in the molar ratio $1: 1$, as compared to the sum of the effects of the individual agents at the same concentrations as used in combination. The synergy of thrombolytic action of this combination maintained within $24 \mathrm{~h}$ and was more pronounced at the subsequent addition of scu-PA following SK (in $1 \mathrm{~h}$ ). At the concentrations of both plasminogen activators $\geqslant 25 \mathrm{nM}(\geqslant 100 \mathrm{U} / \mathrm{ml})$ the synergistic thrombolytic effect was accompanied by the marked decrease in plasminogen, $\alpha_{2}$-antiplasmin and fibrinogen concentrations in the surrounding plasma. We conclude, therefore, that SK and Scu-PA do act synergically on clot lysis in a human plasma system in vitro.

Lab. Blood Coagulation, National Haematological Scientific Center, Novo-Zykovski pr. 4a, Moscow 125167, Russia

\section{Malignancy and Haemostasis}

\section{5}

Does leukocyte endothelial cell interaction influence the hemostatic balance in acute leukemia?

T Südhoff, A Wehmeier, B Bauser, P Schlömer and W Schneider

Department of Hematology, Oncology and Clinical Immunology, HeinrichHeine-University, Moorenstr.5, 40225 Düsseldorf

Hemostatic disorders are frequently found in patients with newly diagnosed acute leukemia (AL). in AL, endothelial cells (EC) and blast cells have been shown to contribute to regulation of clot formation and lysis. Recently, leukocyte endothelium interaction via adhesion molecules has been a subjet of intensive investigation. Like neutrophils, blast cell adherence to $E C$ is primarily mediated by E-selectin and vascular adhesion molecule-1 (VCAM-1) which are expressed exclusively on endothelial surface and finally can be detected in the circulation as soluble isoforms.

If adhesive interactions between leukocytes and $\mathrm{EC}$ are increased in $\mathrm{AL}$, it will be conceivable that these mechanisms may influence regulation of the hemostatic balance. To test this hypothesis, we analyzed plasma concentrations of E-selectin and VCAM-1 molecules in 37 patients with AL (acute myeloid leukemia $=31$, acute lymphoblastic leukemia $=6$ ) with normal renal and liver function on admision by commerically available ELISA-assays.

As compared with 60 healthy controls $(40 \pm 15 \mathrm{ng} / \mathrm{ml}$ and $930+220$ $\mathrm{ng} / \mathrm{ml}$, respectively) both, E-selectin $(86 \pm 89 \mathrm{ng} / \mathrm{ml})$ and VCAM-1 $(1900 \pm 1470 \mathrm{ng} / \mathrm{ml})$ were significantly elevated with high interindividual variability.

Concering hemostatic parameters, decreased prothrombin time $\quad<75 \%$, $n=17)$ was accompanied by elevated E-selectin plasma levels (120 \pm 116 vs $57 \pm 45, p<0.05)$. When $D$-dimer levels were determined $(n=17)$, elevated $D$-dimers ( $>3 \mathrm{mg} / \mathrm{l}, \mathrm{n}=7$ ) paralleled increased E-selectin concentrations $(128 \pm 44$ vs $46 \pm 21 \mathrm{ng} / \mathrm{ml}, \mathrm{p}<0.01)$. In contrary, no significant differences in VCAM-1 levels could be detected.

in conclusion, these data indicate increased interaction between blast cells and endothelium in the majority of $\mathrm{AL}$ as shown by elevated circulating $E$ selectin and VCAM-1 molecules. Correlation of E-selectin plasma levels with decreased prothrombin time and increased D-dimer concentrations suggests that adhesive mechanisms result in functional changes in these two cell compartiments and thus may contribute to distrubances in the hermostatic balance in patients with overt $\mathrm{AL}$.
THROMBOSPONDIN AND OTHER PLATELET-DERIVED MOLECULES IN PLASMA OF PATIENTS WITH CARCINOMA OF THE UTERUS R. Mücke, M. Steiner\#, J. Schmelzer \#, C. Burstein $\#$, M. Strietzel B. Ernst ${ }^{\#}$

Adhesion molecules of different composition and origin are involved in tumor progression and metastasis. Platelet $\alpha$-granules contain large amounts of thrombospondin (TSP) which is recently being associated with malignancy. In addition, plasma TSP has been suggested to be a good candidate for a tumor marker. We have investigated plasma TSP in 11 women with metastatic carcinoma of the uterus undergoing radiation therapy. Platelet factor 4 (PF 4) and Bthromboglobulin (B-TG) were included as further $\alpha$-granule-derived molecules. All parameters were investigated in CTAD-anticoagulated plasma using enzyme immunoassays (STAGO). 34 age-matched women served as control group. $70 \%$ of tumor patients demonstrated increased B-TG and PF 4 concentrations indicating platelet activation. Significant correlations between B-TG and PF 4 were found in both healthy controls $(r=0.81)$ and cancer patients $(r=0.82)$. Plasma TSP was found to be elevated in only $30 \%$ of tumor patients with a mean value not significantly different from that of controls. Plasma TSP did not show significant correlations to PF 4 and B-TG in either healthy controls or patients. Due to a significant overlap between controls and women suffering from advanced carcinoma of the uterus plasma TSP can not serve as a tumor marker at least in this type of cancer. Furthermore, the lack of correlation of plasma TSP with other $\alpha$-granule-derived molecules raises the question about extra-platelet sources of plasma TSP.

University of Rostock, Department of Radiology, Division of Radiation Therapy \& Oncology, Institute of Clinical Chemistry \& Pathobiochemistry\#, Südring 75, 18059 Rostock, Germany 


\section{ALL-TRANS RETINOIC ACID TREATMENT IN ACUTE PROMYELOCYTIC LEUKEMIA IS ASSOCIATED WITH THROMBOEMBOLIC EVENTS}

V. Runde, T. Südhoff, C. Aul, M. Heins*, A. Wehmeier, A. Heyll, D. Söhngen. H. Reinauer*, and W. Schneider

Acute promyelocytic leukemia (APL) is a distinct variety of acute myelogenous leukemia characterized by the occurrence of potentially life-threatening hemorrhagic complications. Bleeding results, at least in part, from the release of tissue procoagulant activity but a fibrinolytic/proteolytic activity due to leukocyte proteases has been proposed as an important additional event. Since 1988 it has been reported that treatment with all-trans retinoic acid (ATRA) promotes terminal differentiation of leukemic promyelocytes, resulting in complete remission rates of $65 \%$ to $95 \%$. During the last 3 years, we have treated 8 APL patients (untreated, $n=5$; first relapse, $n=2$; second relapse, $n=1$ ) with ATRA. Five patients showed the characteristic hemostatic disorder with a marked reduction in fibrinogen levels. Two of these patients developed ATRA induced leukocytosis and thromboembolic events resulting in a fatal outcome in one of them. In 2 patients plasmin-alpha 2 antiplasmin complexes (PAP) were measured during the first days of ATRA treatment. It is of note that, even after normalization of $f i$ brinogen levels, continuously high PAP levels were found $(>5,000$ $\mu \mathrm{g} / \mathrm{l}$, normal range: $80-470 \mu \mathrm{g} / \mathrm{l})$. Although this considerable fibrinolytic activity can be detected, based on our clinical experience, we recommend heparinization in ATRA treated APL patients when fibrinogen levels had normalized and thrombocytes are above $50,000 / \mu \mathrm{l}$.

Medizinische Klinik und Poliklinik, Abteilung für Hämatologie Onkologie und klinische Immunologie, *Institut für klinische Chemie, Heinrich-Heine-Universität, Düsseldort, Moorenstr. 5 , 40225 Düsseldorf
PROTEIN $C$ AND PROTEIN $S$ LEVELS IN BONE MARROW TRANSPLANT RECIPIENTS

c. Salat, E. Holler, B. Reinhardt, HJ. Kolb, B. Seeber, T. Duell, E. Hiller

Among thrombotic phenomena in the course of bone marrow transplantation (BMT) as thromboembolism or occlusion of central vein catheters particularly hepatic veno-occlusive disease (VOD) is of clinical significance, since VOD represents one of the three most frequent complications with fatal outcome. To evaluate a possible role of a reduction of natural anticoagulants we measured protein $C$ (PC) and protein S (PS) levels in patients undergoing BMT.

Materials and methods: 32 patients were investigated prospectively. Blood sampling was performed before (day -8 ) and after (day 0 ) conditioning and after BMT (day $7,14,28$ ). Protein $C$ and $S$ were determined by enzymimmunoassays (Asserachrom protein C, ELISA Protein S, Boehringer, Germany) .

Results: 4 patients developed VOD. Posttransplant PC levels were even higher in voD pts. (mean $98,6+/-20,2 \%)$ than in pts. without voD $(72,0+/-$ $12,4 \%)$. PS levels increased after a low on day 7 (VOD $33,2+/-10,7 \%$; no VOD $55,7+/-19,1 \%$ ) in the posttransplant phase (VOD $68,9+/-11,5 ;$ no VOD $74,5+/-4,5 \%)$.

Discussion: Although a remarkable reduction of PS was found particularly in VOD patients in the early posttransplant phase the signicance of this finding remains unclear because levels increased during the following period also in pts. with VOD. Our results speak against a role of a reduction of $P C$ in the pathogenesis of VOD which was reported by others recently.

Med. Klinik III, Klinikum Grosshadern der LudwigMaximilians-Universität München

\section{8}

FACTOR XIII IN LUNG CANCER PATIENTS: CORRELATION WITH TUMOUR STAGE, THROMBIN GENERATION AND NEUTROPHIL ELASTASE $\left(^{*}\right)$

R. Seitz, N. Rappe, A. Immel, H.-H. Heidtmann, M. Maasberg, R. Egbring, K. Havemann

In patients with lung cancer, frequently activation of coagulation and inflammatory reaction with release of neutrophil elastase (HNE) is encountered. An enhanced turnover of F XIII can be due to its activation by thrombin or non-specific proteolysis by elastase. In 57 lung cancer patients, plasma levels of F XIII, thrombin-antithrombin complex (TAT), prothrombin fragment $F 1+2$, and HNE complexed to proteinase inhibitor were determined before antineoplastic therapy.

There were no significant differences between the 19 small celi (SCLC) and the 38 non-small cell (NSCLC) cancer patients. In extensive disease (ED, $n=37)$, TAT $(p=0.0093)$ was higher, and F XIII $(p=0.0531)$ lower than in localised disease $(L D, n=20)$. However, a statistical correlation was only found in ED patients between $F$ XIIl and HNE $(r=$. $0.407, p=0.015$ ). In patients not responding with remission (NRESP, $\mathrm{n}=41)$ to subsequent treatment, TAT $(\mathrm{p}=0.0005)$ and $\mathrm{F} 1+2$ $(p=0.0455)$ were higher than in the responders (RESP, $n=16)$. However, in the NRESP patients, there was a correlation of F XIII only with HNE $(r=-0.494, p=0.001)$.

Thus, activation of coagulation in lung cancer increases with spread of the tumour and deteriorating prognosis. The F XIII plasma level, however, appears to be more influenced by the inflammatory reaction. This would suggest that coagulation takes place within the tumour, while inflammation is a systemic reaction.

Philipps-University Hospitais, Dept. of Haematology, Baldinger Str., D-35033 Marburg, Germany. ( $\left.{ }^{*}\right)$ Supported by Deutsche Krebshilfe.

\section{0}

COAGULATION ACTIVATION IN PATIENTS WITH NON HODGKIN LYMPHOMA DURING POLYCHEMOTHISRAPY

A. Bouka, W. Schroyens, H. Pralle, K.J. Matthes, B. Kemkes-Matthes

INTRODUCTION: Malignant diseases as well as their treatment can induce coagulation activation. To find out, whether polychemotherapy enhances coagulation activation in Non Hodgk in Iymphoma (NHI) patients, we measured coagulation activation markers TAT, D-Dimer, FDP, routine coagulation tests and coagulation inhibitors.

PATTENTS: 9 CHOEP cycles (Cyclophosphamid, Adriamycin, Vincristin, Etoposid, Prednison) in 4 patients suffering from NHI were studied. Blood samples were taken at the $1 \mathrm{st}$, 2nd and 3rd day of therapy immediately before and after infusion.

METHODS: TAT: Enzygnost TAT Mikro, Behringwerke Marburg, Germany; D-Dimer: EIISA Test D-Dimer, Boehringer Mannheim, Germany; FDP: Fibrinostika TDP Mikroelisa, Organon Teknika GmbH, Eppelheim, Germany.

RESULTS: AII Non Hodgkin Lymphoma patients showed increased coagulation activation markers: TAT: $7,5+9,9 \mathrm{ng} / \mathrm{ml}$, D-Dimer: $761 \pm 759 \mathrm{ng} / \mathrm{ml}, \quad$ FDP: $1.274 \pm 568 \mathrm{ng} \mathrm{FE} / \mathrm{ml}$. No significant changes were observed during polychemotherapy. Coagulation inhibitors AT III ad protein C were in the normal range, while free protein $S$ levels were decreased and $C 4 b-$ BP levels were increased.

CONCLUSION: In Non Hodgkin Lymphoma patients elevated TAT, D-Dimer and FDP levels were observed indicating coagulation activation. No significant changes of activation markers were observed during therapy. We conclude that chemotherapy with CHOEPregimen does not further enhance coagulation activation due to the underlying disease.

Zentrum fü.r Innere Medizin der Universität Gießen, Klinikstrasse 36, D-35392 Giessen. 
EFFECT OF RECOMBINANT HUMAN GRANULOCYTE COLONYSTIMULATING FACTOR (rhG-CSF) ON $\propto$-GRANULES OF PLATELETS.

M. Sosada, H. J. Avenarius, F. Bergmann and H. Poliwoda.

Recent reports suggested the activation of platelets by rhG-CSF. Especially the enhancement of $C D 62$, a selectin integrated in $\alpha$ granules membrane, was detected. Hence, we investigated $\alpha$-granules components ( $B$-thromboglobulin (B-TG) and von Willebrand Factor (VWF)) to determine the degree of release reaction. Blood of 10 healthy volunteers $(6$ female, 4 male, mean age 31 , range 20-45 years) was mixed with rhG-CSF in a final concentration of 1 $\mathrm{ng} / \mathrm{ml}$. B-TG and VWF, immunological ( $\mathrm{VWF}$ : $\mathrm{Ag}$ ) and functional activity (RiCof), were measured in native blood and after addition of rhG-CSF. Results were as follows:

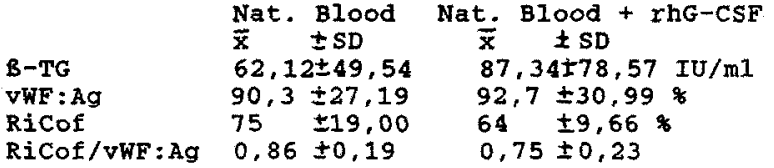

After stimulation with rhG-CSF we observed a significant increase of $B-T G \quad(2 p<0,05)$. Interestingly, vWF parameters as well as multimeric analysis did not reflect any changes. This might be due to binding of released $\mathrm{VWF}$ to platelets membrane. On the other hand the amount of released VWF is probably too small to be detected in plasma. The clinical relevance of observed B-TG release has to be determined.

Div. of Hematology and Oncology, Hannover Medical School. D-30623 Hannover

\section{2}

COAGULATION DISORDERS IN THE PREPARATIVE PERIOD IN BONE MARROW TRANSPLANTATED (BMT) PATIENTS

I.V.Tamarin*, L.A.Scherbinina, G.V. Bashkov

BMT is an accepted treatment now for patients with hematological malignancies and is often accompanied by thrombotic and bleeding complications. The purpose of this study was to investigate the state of blood coagulation before, during preparative period and in the early phase after were included in this study. The conditioning regimen was Cyclophosphamide and total body irradiation (TBI, 6 pts) or chemotherapy only ( 9 pts). All patients received low-dose heparin prophylaxis of venoocclusive disease. Most of the patients had initial liver failure with the decrease of protein $\mathrm{C}$ (PC) activity (mediana-60\%) and elevation of thrombin and prothrombinase activities as judged by an increase in fibrinopeptide A (FPA, mediana-4,1 $\mathrm{ng} / \mathrm{ml}$ ) and thrombin-antithrombin III complex (TAT, mediana-5,2 $\mu \mathrm{g} / \mathrm{l}$ ) concentrations. It can be connected with the previous chemotherapy of the hematological malignances, the disease itself and/or a history of viral hepatitis. The preparative treatment caused the endothelial damage with the concominant von Willebrand factor liberation $(\mathrm{P}<0,001)$ with a short-term fibrinolysis activation $(\mathrm{P}<0,05)$ due to tissue-type plasminogen activator release. We detected the significant simultaneous increase in thrombin generation measured by the rise in FPA $(\mathrm{P}<0,01)$. TBI caused the more pronounced changes in hemostasis than chemotherapy. At the end of preparative period $\alpha_{2}$-antiplasmin and fibrinogen levels were elevated $(P<0,01)$ and fibrinolysis was depressed $(\mathrm{P}<0,05)$. At this time the increase in $\mathrm{PC}(\mathrm{P}<0,05)$ and antithrombin III (AT, $\mathrm{P}<0,01$ ) activities were detected. We suggest that the endothelial damage in preparative period induces the alterations of thrombin-dependent clearence of PC and AT from the blood stream. These findings can contribute to an increased risk of coagulation complications in BMT.

Present address: Laboratory of Clinical Coagulology, National Hematological Center, Novozykovsky pr. 4, 125167 Moscow, Russia

\section{Endothelial Cell}

\section{3}

THE COMPLEXITY OF THE ENDOTHELIAL CELL RESPONSE TO TNF INCLUDES SUPPRESSION OF NOVEL GENES INCLUDING UBIQUITIN, FIBRONECTIN AND FISP-12

J. Lin, B. Liliensiek, M. Kanitz, B.-S. Chen, R. Ziegler, P.P. Nawroth

TNF is a cytokine that appears to orchestrate the inflammatory response. One target is the vascular endothelium. Stimulation of endothelial cells by TNF has been viewed as induction of proinflammatory reactions, the only exceptions known are thrombomodulin and the NO-synthetase. Given the diversity of cellular responses to cytokines, not all effects of TNF can be understood on the basis of induced synthesis and expression of new proteins. Therefore it seems likely that a number of unidentified genes might be suppressed by TNF. To characterize the genes encoding TNF suppressed proteins we have used the technique of differential screening of a subtractive endothelial cell CDNA library enriched for TNF suppressed genes. We now report the initial analysis of 24 genes suppressed by TNE. Of the 24 genes identified 19 encode hitherto undescribed TNF response genes. Of the others FISP-12 is a growth related gene, fibronectin involved in regulation of vascular permeability and ubiquitin in regulating the halflive of growth factors.

This shows that the endothelial cell response to TNF does not only consist of nonspecific gene induction. In contrast the identification of 24 suppressed genes suggests a well controlled cellular response consisting of gene induction and suppression. The characterisation of these genes should provide new insights into the mechanism by which TNF excerts its pleiotropic effects on endothelium.
244

APOLIPOPROTEIN (A) MEDIATES THE INTERACTION OF LIPOPROTEIN (A) WITH THE LOW DENSITY LIPOPROTEIN RECEPTOR-RELATED PROTEIN/ $\alpha_{2}$-MACROGLOBULIN RECEPTOR

A. Beckmann 1 , W. März ${ }^{2}$, H. Scharnagl'1, R. Siekmeier' ${ }^{1}$, U. Mondorf ${ }^{3}$, H. Wieland ${ }^{2}$, and W. Groß 1

Lipoprotein (a) (Lp(a)) is a complex of low density lipoprotein (LDL) with apolipoprotein (apo) (a). We previously showed that $\operatorname{Lp}($ a) particles are heterogenous in size, displaying apparent molecular masses $(M)$ of $2 \cdot 10^{6}$ to at least $10 \cdot 10^{6}$. Lp(a) size isoforms differed by the expression of apolipoprotein B epitopes and their interaction with cultured human skin fibroblasts. Low $M_{t} L p(a)$ was internalized by $L D L$ receptors. In contrast, high $M_{r} L p(a)$ was taken up by the $L D L$ receptor related protein $/ \alpha$ macroglobulin receptor (LRP/AMR) (März et al. FEBS Lett 325, 271-5, 1993). To further characterize the molecular mechanisms of the interaction between $L p(a)$ and LRP/AMR, we studied binding and uptake of $L p(a)$ size isoforms in normal and in FH fibroblasts (less than $10 \% \mathrm{LDL}$ receptor activity). In both cell types, tissue-type plasminogen activator and activated $\alpha_{2}$-macroglobulin competed with the uptake of high $M_{r} L_{p}(a)$. The $39 \mathrm{kD}$ LRP/AMR associated protein (RAP) inhibited binding, uptake and degradation. Adducts consisting of recombinant apolipoprotein (a) and LDL exhibited increased binding (7-fold), uptake (2-fold), and degradation (2-fold), compared to the LDL core particle. The $39 \mathrm{kDa}$ RAP completely blocked the apo(a) mediated increases in uptake and degradation, but only partially blocked the increase in binding. The apo(a) specific monoclonal antibody $\mathrm{cl} 280$ inhibited binding, uptake, and degradation of high M $L p($ a) by $80 \%, 50 \%$ and $40 \%$, respectively, but it had no effect on the uptake of low $M_{r} L p(a)$. The apo(a) specific monoclonal antibody $\mathrm{cl} 356$ had no effect on the interaction between $L p(a)$ and LRP/AMR. We conclude from these data a) that the interaction of high $M_{r} L p(a)$ with LRP/AMR is mediated by apo(a); b) that the uptake of high $M_{-} L p(a)$ almost completely proceeds via LRP/AMR c) that binding sites for high $\mathbf{M}_{\mathrm{r}}$ $L p(a)$ exist on the cell surface that are not involved in internalization and degradation, and d) that domains defined by the cl280 epitope(s) contribute to the binding of $\mathrm{HM}_{\mathrm{r}} \mathrm{Lp}(\mathrm{a})$ to $\mathrm{LRP} / \mathrm{AMR}$.

${ }^{1}$ Center of Biological Chemistry and ${ }^{3}$ Center of Internal Medicine, J. W. Goethe-University, Frankfurt, and 2Department of Clinical Chemistry, Albert Ludwigs-University, Freiburg 
GROWTH AND FIBRINOLYTIC PARAMETERS OF CULTURED HUMAN UMBILICAL VEIN ENDOTHELIAL CELLS ON ARTIFICIAL SURFACES

Jingchuan Zhang, Johann Wojta and Bernd R. Binder

Although blood compatibility of biomaterials improved over the years, a nonthrombogenic surface could not be found. Therefore the idea to seed endothelial cell (EC) on the luminal side of cardiovascular grafts became increasingly attractive. It was the aim of this study to compare EC growth on different biomaterials and to use tissue type-plasminogen activator (t-PA) and plasminogen activator inhibitor-1 (PAI-1)-production by such EC as a marker for normal EC function. Human umbilical vein EC (HUVEC) were isolated from umbilical cords by mild collagenase treatment and grown in Medium 199 supplemented with $20 \%$ calf serum and endothelial cell growth supplement. HUVEC were seeded at passage 1 or 2 onto polytetrafluoroethylene (PTFE; purchased from W.L. GORE \& Assoc. Austria) or polyurethane (PU; provided by Institute of Chemistry, China) precoated with or without fibronectin (FN) $\left(10 \mu \mathrm{g} / \mathrm{cm}^{2}\right)$ at a density of $9 \times 10^{3}$ cells $/ \mathrm{cm}^{2} .4$ days after seeding the cells were counted. HUVEC counts increased to $4.3 \times 10^{5} / \mathrm{cm}^{2}$ on PU and to $3.3 \times 10^{5} / \mathrm{cm}^{2}$ on PTFE when coated with FN, respectively $(p<0,01)$ whereas cell numbers increased only to $1 \times 10^{5} / \mathrm{cm}^{2}$ on $P U$ and to $0.15 \times 10^{5} / \mathrm{cm}^{2}$ on PTFE without FN, respectively. 24 hour conditioned media of confluent monolayers of HUVEC grown on PU or PTFE coated with FN, respectively, was collected and tested for the presence of $\mathrm{t}-\mathrm{PA}$ and PAI-1 antigen with specific ELISA's. HUVEC produced $2.24 \pm 0.16 \mu \mathrm{g} / 10^{5}$ cells $/ 24 \mathrm{~h}$ PAI-1 antigen on PU and $2.51 \pm 0.31 \mu \mathrm{g} / 10^{5}$ cells/24h PAI-1 antigen when grown on PTFE. t-PA antigen values were $15.4+1.2 \mathrm{ng} / 10^{5} \mathrm{cells} / 24 \mathrm{~h}$ on $\mathrm{PU}$ and $13.8+$ $1.5 \mathrm{ng} / 10^{5}$ cells $/ 24 \mathrm{~h}$ on PTFE, respectively. In conclusion our results demonstrate that precoating of artificial surfaces with $F N$ increases $E C$ growth significantly. Furthermore the growth rate of HUVEC on FN coated PU was significantly higher than on FN coated PTFE. Finally our data indicates that HUVEC grown on PU and PTFE produce t-PA and PAI-1, two markers of normal endothelial function.

Clinical Experimental Physiology, University of Vienna, Schwarspanierstraße 17, A-1090 Vienna, AUSTRIA

EFFECT OF EXOGENOUS HYPERLIPEMIA ON RABBIT AORTIC ENDOTHELIAL CELL PAI-1 SYNTHESIS

D.B. AL-Azhary, N.S. Tawfek, W.J. Zhang, J. Wojta and B.R. Binder

This study was performed to investigate the effect of exogenous hyperlipemia on PAI-1 production by rabbit aortic endothelial cells (RACEs). The cells were isolated from Male New Zealand White rabbits after feeding them on either normal pellet food or hyperlipemic diets consisting of $2 \%$ cholesterol, $14 \%$ natural fat, $14 \%$ corn oil, and $70 \%$ normal pellets for a period of 28 dayes. RAECs were cultured and grown to confluence in medium with $10 \%$ SCS and washed twice before incubation with serum free conditioned medium (CM) for 16 hours. Plasminogen activator inhibitor-1 activity (PAI-1) in CM and PAI-1 mRNA in cells were analyzed. Furthermore the effect of addition of inactivated hyperlipemic serum and different lipoprotein fractions prepared from plasma of hyperlipemic animals on normal endothelial cells was investigated. RAECs of hyperlipemic animals showed a significant increase in PAI-1 activity in CM and on the level of mRNA expression by the cells. Addition of hyperlipemic serum to cultured normal endothelial cells induced a significant increase in PAI-1 activity in CM. Moreover, both very low density (VLDL) and low density (LDL) lipoproteins from plasma of hyperlipemic rabbits increased PAI-I activity in conditioned media in a dose dependent manner. These results indicate that long-term intake of such a hyperlipemic diet affects PAI-1 production by endothelial cells. The data also give evidence that the levels of VLDL and LDL play a role in PAI-1 synthesis by endothelial cells

Lab. Clin. Exp. Physiology, Department of Medical Physiology, University of Vienna, 'A-1090 Vienna, Austria
INFLUENCE OF CULTURE CONDITIONS ON THE EXPRESSION OF THROMBOMODULIN IN CULTURED HUMAN SAPHENOUS VEIN ENDOTHELIAL CELLS

Anita Raschdorf, M. Keller, T. Schmidt", W.-P. Klövekorn', G. MüllerBerghaus, and B. Pötzsch

Thrombomodulin (TM) is an essential cofactor in thrombin-catalyzed activation of the anticoagulant serine protease protein C. Expression of this endothelial cell-specific glycoprotein has been extensively studied using cultured human umbilical vein endothelial cells (HUVEC). However, concerns were raised whether results obtained with HUVEC reflect the real in vivo situation since the biological function of HUVEC is terminated after birth. To overcome this problem, we established a culture system for human saphenous vein endothelial cells (HSVEC) and analyzed the influence of different culture conditions on the expression of TM. HSVEC were isolated by surface digestion with $0.0125 \%(\mathrm{w} / \mathrm{v})$ collagenase from segments of saphenous veins obtained from patients undergoing aorto-coronary bypass grafting. Among different types and concentrations of sera tested, 30\% heat inactivated human serum was found optimal for cell growth and cell viability. Using these culture conditions HSVECs were cultured up to 10 passages without changing their morphology or losing endothelial cell specific properties. For determination of TM, cells were cultured on microtiter plates, fixed with $1.0 \%$ formaldehyde in PBS, and TM quantified using the monoclonal antibody TME directed against the human recombinant TM derivative solulin. When the cells reached confluency a concentration of 10 $\pm 2.1 \mathrm{ng}$ TM per $40 \mathrm{~mm}^{2}$ cell surface was measured independent of the total cell number. TM concentrations were constant during passages 1-10, not influenced by different culture conditions (growth factors, sera concentration etc.), and identical in HUVEC and HSVEC. Our data indicate that TM levels measured in cultured endothelial cells should be expressed relative to the cell surface and not to the total cell count to avoid misinterpretation.

Abteilung Hämostaseologie und Transfusionsmedizin, and "Abteilung Thorax- und Kardiovaskularchirurgie, Kerckhoff-Klinik, Sprudelhof 11. D-61231 Bad Nauheim.

EFFECTS OF TFPI ON BLOOD COAGULATION IN VITRO AND ON LASER INDUCED THROMBUS FORMATION IN RAT MESENTERIUM

P.Radziwon* W.Weichert,B.Boczkowska-Radziwon, J.Schenk, D. Hoppenstaedt **, J.Fareed ${ }^{* *}$, H.K.Breddin.

Tissue factor pathway inhibitor (TFPI) is a physiological factor $X a$ dependent inhibitor of the factor Vila/tissue factor complex. It is primarily synthesized by endothelial cells. Heparin causes the liberation of TFPI from endothelium and enhances the inhibition of factor $X a$ by heparin. We have studied the inhibitory effecs of recombinant TFPI (rTFPI)(kindly obtained from Dr.Broze,Jr., Washington University Medical Center, St.Louis, USA) on standard coagulation tests (aPTT, PT, Heptest, ATIII) using an automated analyser (ACL, Italy), on platelet induced thrombin generation time (PITT) - a newly developed global coagulation assay (Haemostasis 1992; 22: 309-321), on platelet adhesion and on laser induced thrombus formation in the mesenteric microcirculation of rats. We observed a strong, dose dependent and statistically significant intibition of PTT and also of aPTT, PT and Heptest in a dose range from $0.1 \mu \mathrm{g} / \mathrm{ml}$ to $1 \mu \mathrm{g} / \mathrm{ml}$. All these tests detect the effect of TFPI on blood coagulation with a similar sensitivity. $30 \mathrm{~min}$ after iv injection of $10 \mu \mathrm{g} / \mathrm{kg}$ we observed statistically significant inhibition of thrombus formation in rat mesenteric venules. TFPl is an effective physiologic agent in vitro and in vivo and as that schould be considered as a new interesting anticoagulant and possibly also an effective antithrombotic agent.

Dept. of Internal Medicine, Division of Angiology, J.W.GoetheUniversity, Frankfurt am Main, Germany.

* Dept. of Haematology, Medical School, Bialystok, Poland,

* * Hemostasis Research Laboratories, Loyola University medical Center, Maywood, USA 
TISSUE FACTOR (TF) AND TISSUE FACTOR PATHWAY INHIBITOR (TFPI) THEIR ROLES IN THROMBOTIC ACTIVATION AND THERAPEUTIC INTERVENTIONS. D. Callas, J. Fareed, D. Hoppensteadt, W. Jeske S. Moran, and E.W. Bermes. Loyola University Medical Center, Maywood, IL. 60153.

It is now generally agreed that surgical procedures and cardiovascular interventions produce significant tissue damage resulting in localized and generalized release of TF. This agent, through cellular and plasmatic activation processes, promotes thrombogenesis. TF is also capable of upregulating such mediators as endothelin, thromboxane $B_{2}$ and plasminogen activator inhibitor. Thus, besides promoting protease generation, TF has several other target sites involving organ/cells, blood vessels, platelets and neutrophils. The various activities of TF are controlled by another endogenous, newly identified Kunitz type antiprotease, known as TFPI. Most of the TFPI is bound to plasmatic and cellular sites. Polyelectrolyte drugs such as heparin are capable of releasing this inhibitor into the circulation. Thus, the plasmatic level of TFPI increases during heparin therapy although levels differ markedly during therapeutic and prophylactic treatment. From a diagnostic standpoint both TF and TFPI may be important in the assessment of a clinical state related to a thrombotic event. While TF is not detectable in a normal state, following surgery minute changes in the plasma amounts can be found. However, at the surgical site, in open wounds, or at traumatic areas markedly increased levels of this marker have been detected. Significant amounts of TF have been found in spinal fluid, bronchial lavage, synovial fluid and urine. Thus, the extent of tissue damage or thrombotic risk may be identified based on TF levels. The availability of ultrasensitive methods to quantitate picogram levels of both TF and TFPI therefore, may provide a unique approach to assess the patient's risk or the therapeutic effects of drugs. With the availability of antibodies to both TF and TFPI, additional diagnostic methods such as immunochemical mapping and diagnostic imaging may be possible.

\section{IMMUNOSTAINING OF TISSUE FACTOR PROTEIN IN PARAFFIN EMBEDDED HUMAN TISSUE SPECIMENS}

C. Flössel, T. Luther, M. Kasper, S. Albrecht, and M. Müller

Tissue factor (TF), a transmembrane glycoprotein of $47 \mathrm{kDa}$, is the essential receptor and cofactor for factor VIIa catalyzing the activation of factors IX and $\mathrm{X}$, thus triggering the extrinsic pathway of coagulation cascade. Recently, the distribution of TF in normal human tissues (Drake TA et al., Am. J. Path. 134:1087, 1989) and in solid tumours (Callander NS et al., Cancer, 70:1194, 1992) has been delineated. The immunostaining of fresh frozen human tissues revealed abundant TF expression in epithelial tissues, brain, kidney, pneumocytes, placenta and adventitia of arteries, but not in endothelium and circulating blood cells. Nevertheless, in frozen sections the exact cellular definition remained unsatisfying, at least in certain tissues. Using a cocktail of three monoclonal antibodies against TF, generated in our laboratory (Albrecht $S$ et al., Blood Coagul. Fibrinol. 3:263, 1992), we can show an exellent immunostaing of TF protein in formalin fixed and paraffin embedded microwaved tissue specimens. This technique at the first time provides the exact distribution of $T F$ in normal and malignant tissues and, in most cases, its precise cellular localization. On the other hand, this system allows retrospective investigations in a wide range of archival histological material. We postulate that such investigations will contribute to a better understanding of biological role of TF in the organism, because data on structural and functional biology of TF suggest that it may participate in processes other than hemostasis. Human tissue distribution and cellular localization of TF including examples of pathological settings will be presented.

Technical University of Dresden, Institute of Pathology, Fetscherstraße 74, 01307 Dresden, Germany

INHIBITION OF THROMBIN AND FACTOR Xa GENERATION BY TISSUE FACTOR PATHWAY INHIBITOR (TFPI) - MODULATORY EFFECT OF GLYCOSAMINOGL YCANS AND A POLYSULFONATE

B. Kaiser, D. Hoppensteadt ${ }^{\star}$, W. Jeske* , T.C. Wun ${ }^{\star *}$ and J. Fareed

The effect of TFPI (Monsanto, St. Louis, MO), heparin (UFH), low molecular weight heparin (LMWH), heparan sulfate (HS) and a polysulfonate (GL-522) on protease generation was studied in an in vitro assay system using the prothromb in complex concentrate Konyne $(R)$. The amount of thrombin and factor $X a$ generated was measured with chromogenic substrates on a microcentrifugal analyzer After activation of coagulation with tissue factor (Dade $(\hat{R})$ InnovinTM, Miami, FL) TFPI caused a concentration-dependent inhibition of both thromb in (IC $50=$ $255 \pm 38 \mathrm{ng} / \mathrm{m} 1)$ and factor $X_{a}\left(\mathrm{IC}_{50}=684 \pm 18 \mathrm{ng} / \mathrm{m} \mathrm{T}\right)$ generation. In the presence of UFH $(10 \mu \mathrm{g} / \mathrm{m} 1)$ the TFPI-induced inhibition of protease generation was still increased (IC50 for thromb in generation $=116 \pm 9 \mathrm{ng} / \mathrm{ml}$; I I 50 for factor $X_{a}$ generation $=257 \pm 12 \mathrm{ng} / \mathrm{ml}$ ). Using a subthreshold concentration of TFPI which did not inhibit protease generation by itself, the effect of UFH, LMWH, HS and GL-522 on thromb in and factor $X a$ generation was studied. Except $\mathrm{GL}-522$, up to a concentration of $40 \mu \mathrm{g} / \mathrm{ml}$ UFH, LMWH and HS did not influence protease generation. However, when the assay system was supplemented with TFPI thromb in and factor $x a$ generation was inhibited by UFH, LMWH and HS up to 40-50\% and the GL-522-induced protease generation inhibition was further increased by about $10-20 \%$.

The studies show that TFPI strongly inhibits thrombin and factor $X_{a}$ generation in an in vitro assay system and that it can increase the inhibitory potency of various anticoagulants/antithrombotics on the generation of serine proteases.

* Department of Pathology, Loyola University Medical Center, Maywood, IL, USA

* Monsanto, St. Lou is, MO, USA

Institute of Pharmacology and Toxicology, Medical School, Erfurt, Germany
CHANGES IN THE METABOLIC ACTIVITIES OF CULTURED ENDOTHELIAL CELLS INCUBATED WITH SERA OF DIABETIC AND CIRPHOTIC PATIENTS

I. Altorjay, M. Udvardy and M. Káplár

High plasmatic Willebrand factor level is considered as a sign of endothelial damage. High levels are found both in diabetic and cirrhotic patients, however atherosclerotic alterations are more frequent in the previous group. In order to compare the effect of sera from the two patient-groups, confluent human cultured endothelial cells were incubated with media containing $20 \%$ diabetic, or cirrhotic as well as control sera for 48 hours. Metabolic activity of the monolayer was measured by the decrease of the glucose content of the medium, while changes of Willebrand factor and LDH content, as well as prostagland in (PGF $7_{\alpha}$ ) production and changes of the antiaggregatory capability of the endothelial cells were appropriately recorded. Impaired metabolic activity under the influence of diabetic - especially IDDM - sera could be established by the decreased glucose consumption and the decreased PG production, leading to decreased antiaggregatory capacity against collagen induced platelet aggregation in contrast to cirrhotic sera, in the presence of which both metabolic activity and Willebrand factor production was found to be somewhat increased. Any direct damaging effect could be excluded by the unchanged IDH levels during the investigation. According to our observation diabetic serum itself and not only high glucose levels may cause impaired endothelial function.

2nd Dept. Med. Univ. Med. School

H-4012 Debrecen, POB. 20. 
RECOMBINANT HUMAN SOLUBLE AND FULL-LENGTH THROMBOMODULIN: EXPRESSION, PURIFICATION AND PRELIMINARY CHARACTERIZATION

M. Steiner, B. Ernst, W. Northemann\#

Thrombomodulin (TMD) is a high-affinity thrombin receptor located predominantly at the luminal endothelial surface. Its main function is to block procoagulant properties of thrombin and upon complexing with thrombin to accelerate protein $\mathrm{C}$ activation thus further contributing to inhibition of coagulation. Therefore, recombinant human TMD is an attractive candidate molecule for an anticoagulant. A recombinant baculovirus construct pTMDS 8 was designed to direct the expression of recombinant human soluble TMD in infected S $f 9$ insect cells. The soluble TMD consisting of 513 amino acids lacks the C-terminal transmembrane region. It carries a C-terminal histidinehexapeptide as affinity ligand for metal-chelating affinity chromatography for purification purposes. Full-length recombinant TMD was expressed in a similar way except it consists of 575 amino acids. Both forms of recombinant TMD could be expressed in large amounts and purified to homogeneity. Analysis of functionality demonstrated dose-dependent inhibition of thrombin procoagulant activities (fibrinogen clotting) with a maximum being observed at eightfold excess of recombinant TMD. Protein $C$ activation occurred at the complex thrombin-recombinant TMD. The data suggest that recombinant TMD is functionally active and could provide a promising alternative to existing molecules with anticoagulant activity. However, further modifications of the recombinant TMD appear to be useful in terms of stability and functionality.

University of Rostock, Institute of Clinical Chemistry \& Pathobiochemistry, Heydemann-Str. 6, 18055 Rostock, Germany, ELIAS GmbH, Department of Molecular Biology, 79114 Freiburg, Germany

Prethrombotic State

COAGULATION ACTIVATION MARKERS IN ACUTE RENAL FAILURE (ARF)

\section{Stefanidis, J. Hägel, and N. Maurin}

Fibrinopeptide $A$ and B-thromboglobulin are used in vivo as markers for enhanced thrombin activity or platelet activation. A pronounced increase in these parameters is observed during ARF. Since renal insufficiency disturbs elimination of these markers, it is necessary to ask whether the enhanced concentration actually indicates hypercoagulability during ARF. In order to check the plausibility of the above-mentioned coagulation markers, we compared fibrinopeptide $A$ and $B$ thromboglobulin concentrations with the thrombin-AT III complex, whose catabolism and excretion are uninfluenced by kidney function, in 32 patients with ARF. Prior to renal replacement therapy, fibrinopeptide $\mathrm{A}(34 \pm 22 \mathrm{ng} / \mathrm{ml}$, ref. $<$ 3.0), thrombin-AT III complex $(19 \pm 15 \mathrm{ng} / \mathrm{ml}$, ref. 1.0-4.0) and B-thromboglobulin (149 $\pm 58 \mathrm{IU} / \mathrm{ml}$, ref. 10-40) were all raised. In cases with DIC $(n=7)$, there was a significantly greater increase in all parameters (fibrinopeptide $A: 44 \pm 28$, Bthromboglobutin: $168 \pm 55$, thrombin-AT IH complex $39 \pm 21$, $\mathrm{p}<0.05)$. There was no correlation with serum creatinine. Fibrinopeptide A was correlated with the thrombin-AT III complex both before $(34 \pm 22, r=0.34, p<0.05)$ and during renal replacement therapy $(23 \pm 18, r=0.57, p<0.001)$. Thrombin-AT III complex was correlated with Bthromboglobulin $(r=0.39, p<0.05)$ and with the $B-$ thromboglobulin/creatinine ratio $(0.50 \pm 0.30, r=0.72, p<$ $0.001)$. Fibrinopeptide $A$ and the B-thromboglobulin/creatinine ratio can be employed in combination with the thrombin-AT III complex as a marker for coagulation activity in ARF. There is no direct relationship between restricted renal function and the plasma concentrations of these parameters, which behave similarly despite their varying elimination patterns.

Medical Clinic II, University Hospital Aachen, D-52057 Aachen, Germany
255

PROTEINASE INHIBITOR COMPLEXES IN THE DIAGNOSIS OF CARDIOGENIC AND SEPTIC SHOCK

R. C. Funck, W. Cassel, H. Blanke, R. Seitz, R. Egbring, B. Maisch*

In pts with circulatory shock, reduced myocardial function and fever the discrimination between cardiogenic (CAS) and septic shock (SES) may be difficult. We determined the plasma concentrations of the proteinase inhibitor complexes elastase-a1proteinase-inhibitor (ELPI) and thrombinantithrombin III (TAT) in pts with both disorders.

Methods: During the first 4 days after onset of shock plasma concentrations of ELPI and TAT were measured in 34 pts with CAS and $15 \mathrm{pts}$ with SES. Values $>700 \mu \mathrm{g} / \mathrm{l}$ were considered to be specific for SES, those $<700 \mu \mathrm{g} / \mathrm{for}$ CAS.

Results: ELPI was significantly higher $(p<0.001)$ in SES than in CAS (table). Based on the $700 \mu \mathrm{g} / /$ ELPI threshold sensitivity was 0.966 in CAS and 0.917 in SES. Specificity was 0.917 in CAS and 0.966 in SES. Neither fever in CAS nor the cause of SES (grampositive of gramnegative bacteria) were of relevant effect on these results. TAT plasma concentrations extremely elevated in both goups but not different in CAS and SES.

\begin{tabular}{|ccc|}
\hline \multicolumn{4}{|c|}{ Mean ELPI Values and 95\%-confidence intervals *: } \\
time after onset of shock [h] & CAS & SES \\
\hline $4-12$ & $411,0 \pm 344,4$ & $2384,0 \pm 444,7$ \\
$12-24$ & $373,4 \pm 356,5$ & $1746,5 \pm 471,6$ \\
$24-48$ & $297,1 \pm 385,1$ & $1983,4 \pm 421,9$ \\
& & *normal value: $<200 \mu \mathrm{g} / \mathrm{l}$ \\
\hline
\end{tabular}

Conclusion: The determination of ELPI is a simple, noninvasive and effective way to discriminate CAS and SES. Pts. with ELPI concentraions $>700 \mu \mathrm{g} / \mathrm{l}$ are very likely to have a septicaemia. Sensitivity and specificity are high. The determination of the TAT - complex does not contribute to the discrimination of CAS and SES.

*Present address: Department of Cardiology, University of Marburg, Zentrum für Innere Medizin, Baldinger Str., 35033 Marburg, FRG 
TAT A PROCNOSTIC FACTOR OF ACUTE PANCREATITIS P. Born, I. Spannagi, M. Classen and R. Lorenz

Time and indication of invasive therapy like endoscopic papillotomy in acute pancreatitis is still controversely discussed and seem to depend from the prognosis. But it is often difficult to evaluate the prognosis. As TAT elevation was seen after ERCP in correlation with inflammatory signs of acute pancreatitis (aP) we wanted to check whether there is a connection between TAT, determined at patients" admittance and the outcome of patients with aP (staged according to Ranson"s score).

In 23 patients $(15 \mathrm{~m}, 8 \mathrm{f}$; age 56ys, range 23-85) with aP of different pathogenesis initially TAT and fibrinogen were measured and compared with the clinical outcome. Uncomplicated (u) course was defined by clinical and chemical improvement within 1 week, while complicated (c) course was characterized by longer duration and/or complications.

All patients but 1 (sudden cardiac death) survived. According to Ranson"s criteria the staging value was low to moderate. In group u mean TAT was $5,6 u a / 1+1-4$. median 3,1 . while in grnup $c$ it was $14,8+1-13,3$ ug $/ 1$, median 6,3 . Sensitivity of TAT according to the patients" outcome was $90,3 \%$, specificity $7 \cdot 2,9 \circ$. The positive predicting value was $76,9 \%$, the negative predicting value $88,9 \%$. Leucocytes ( $u: 9000$ vs $c: 12500)$ and fibrinogen $(u: 528 u g / d l$ vs $c: 780 u g /$ dI) showed a similiar rise.

Conclusion: TAT seems to be of prognostic value for the general situation of patients suffering from acute pancreatitis, but further investigations are absolutely necessary before invasive therapeutic indications can be derived.

11. Medizinische Klinik, Klinikum r.d. Isar, TU München Ismaningerstr. 22

81675 München
Markers of ongoing haemostatic activation in relation to coronary, peripheral and cerebral vascular disease B. Betzl, S. Marzoll, M. Spannagl, W. Schramm, F.A. Spengel

Deposition and impaired removal of fibrin is considered as a precipitating event in vascular disease. We studied 101 consecutive patients admitted to a rehabilitation center for recovery from vascular, myogenic or valvular cardiac disease. Plasma samples were investigated for markers of ongoing coagulation ( $F 1+2$ fragment, Thrombin-Antithrombin (TAT) complex, Fibrin-Monomer) and fibrinolysis (Plasmin-Antiplasmin complex, D-Dimer, t-PA, PAI I). Individual plasma levels were compared with angiographic and ultrasound examinations of coronary, cerebral and peripheral arteries. The investigations were performed not earlier than 3 weeks after any vascular event.

We found significantly elevated plasma levels of TAT complex (3.7(1.8) vs. $2.6(1.5) \mathrm{ng} / \mathrm{ml} ; \mathrm{p}<0.001)$, fibrin monomer (7.1(8.1) vs. $3.8(5.1) \mathrm{ug} / \mathrm{ml} ; \mathrm{p}<0.01$ ) and D Dimer (705(607) vs. 388(326) ng/ml); p<0.001) (mean(SD)) in patients with a pathologic Doppler ankle/arm ratio. PAI I and t-PA levels were significantly correlated with hyperlipoproteinemia. Fibrin monomer as well as D-Dimer plasma levels were found positively correlated to the extent of the vascular occlusive disease.

In conclusion the retrospective design does not allow to ascertain whether high plasmatic levels of activation markers are cause or consequence of vascular occlusive disease. However, markers of ongoing activation of haemostasis might be useful for individual adjustment of therapy.

Abteilung Angiologie der Klinik Höhenried der LVA und der Medizinischen Poliklinik

Haemostaseologische Abteilung, Medizinische Klinik

Klinikum Innenstadt der Universität München
Stable oral anticoagulation suppresses fibrinogen/fibrin conversion without difference between low-dose and high-dose therapy.

Trauner A, Sedlmeier $M$, Goedde $M$, Mosavi $S^{*}$, Stiegier $H^{*}$, Spannagl M, Schramm, $W$

Soluble fibrin and D-Dimer indicate fibrin formation and lysis. In a prospective randomised study we examined the effect of coumarin on these parameters under stable low-dose anticoagulation (LD) versus high-dose anticoagulation (HD).

patients selection: We followed up 26 patients with deep vein thrombosis of the legs over 6 months. 15 patients (mean age $51 \pm 14,3$ years, 6 male 9 female) were adjusted to INR-values between $1,5-2,5$ (LD), 11 patients (mean age $54,9 \pm 15,2,4$ male 9 female) to iNR-values between 2,5-4(HD).

methods: We determined prothrombin time and fibrinogen by routine clotting assays, soluble fibrin (SF) and D-Dimer with Elisa (Böhringer Mannheim) before anticoagulation and $10 \pm 3$ days, 3 and 6 months atter beginning.

results: After 10 days therapy INR was significantly different in LD $(2,23 \pm 0,47)$ vs $\operatorname{HD}(2,9 \pm 0,45)(0<0,0001)$. Regarding the other parameters there was no significant difference in cumulative course of therapy. After $10 \pm 3$ days SF-levels significantly decreased from $29,2+21,5 \mu \mathrm{g} / \mathrm{ml}$ (LD) $/ 31,8 \pm 23 \mu \mathrm{g} / \mathrm{ml}$ (HD) to $7,0 \pm 10,4 \mu \mathrm{g} / \mathrm{ml}$ (LD)

$18,2 \pm 6,5 \mu \mathrm{g} / \mathrm{ml}$ (HD). 3 months after, SF-levels were suppressed to the lower detection range of the test system in both groups $(0,15 \pm 0,28 \mu \mathrm{g} / \mathrm{ml}, p=0,005$ (LD), $0,81 \pm 1,73 \mu \mathrm{g} / \mathrm{ml}, p=0,0006$ (HD).) D-Dimer levels were well correlated.

conclusions: Patients under low and high-dose oral anticoagulation over a stable period of 6 months exhibited a significant decrease in parameters of fibrinogen-fibrin conversion and fibrin degradation in plasma. There was no significant difference regarding high and low- dose therapy. Unexpected a suppression of fibrinogen- fibrin conversion to normal range is not yet reached after $10 \pm 3$ days of coumarin therapy.

Abteilung für Hämostaseologie, Med. Klinik, Klinikum Innenstadt der Universităt München, Zlemssenstr.1, D-80336 München, *Angiologische Abteilung. Städtisches Krankenhaus München Schwabing, Kölner Platz 1, D-80804 München

\section{Markers of ongoing haemostatic activation after coronary stent implantation}

\author{
M.Sedlmeier, M. Spannagl, A. Trauner, \\ H. Mudra, V. Klauss, W. Schramm \\ Medizinische Klinik, Klinikum Innenstadt, Universität \\ München, Ziemssenstr.1, D-80336 München
}

The problems concerning reocclusion in patients with coronary artery disease (CAD) after intracoronary stent implantation have not been resolved. Up to now there is no generally accepted concept of anticoagulation. Furthermore, the effect of the postinvasive increase in fibrinogen and plasminogen-activator-inhibitor (PAD) during the "acutephase-reaction " and the consequences for the hemostatic balance have to be elucidated.

We investigated the course of haemostatic parameters from 12 patients suffering from CAD within two weeks after intracoronary stent implantation : fibrinogen, fibrinmonomer, prothrombin fragments $(\mathrm{F} 1+2$ fragments), thrombin-antithrombin-complex (TAT-complex) - representing the coagulation system - and tissue-plasminogen- activator (t-PA), PAI-I, plasmin-antiplasmin-complex - representing the fibrinolytic system . Citrated blood samples were taken once or twice daily at standardizid time. The anticoagulant therapy consisted of combined application of phenprocoumon, heparin and acetylsalicylacid. Markers of ongoing thrombin-activity (TAT, F1+2-fragments) were not related to "acutephase reaction" demonstrated by increase in fibrinogen and PAI-I.

Peak levels of the "acute-phase"-reactants could be demonstrated 3 to 5 days after intracoronary stent application.

In contrast to fibrinmonomer plasma levels of F1+2-fragments and TATcomplex showed a significant negative correlation with prothrombin time We conclude, that the single assessment of prothrombin time or TATcomplex and $\mathrm{F} 1+2$-fragments does not reflect the present activity of coagulation on the level of fibrinogen/fibrin conversion. 
VARIATIONS OF DIFFERENT HEMOSTATIC PARAMETERS IN KIDNEY TRANSPLANTED PATIENTS BEFORE AND AFTER SURGERY

H.J.Siemens ${ }^{2}$, A.Feddersen, P.Faerber, S.Brückner ${ }^{2}$, R.Winterhoff, E.Schulz, T.Wagner ${ }^{2}$, K.Sack

In recent studies we investigated dialysis patients with recurrent shunt closures in comparison to those without shunt problems and controls with regard to differences in various thrombophilic parameters. In a further research project we determined activities and concentrations of different procoagulatory and fibrinolytic factors before and after shunt preparation as well as before and after kidney transplantation.

In a prospective study 21 coagulatory parameters were investigated in 101 patients (male 55). Compared to controls all other collectives showed an activation of coagulation and decreased fibrinolytic activity in many parameters. Furthermore comparison of the two collectives before and after shunt preparation showed significant differences in the following parameters: t-PA (mean: 8.9/18.4 $\mu \mathrm{g} / \mathrm{L}$ ), prothrombin fragments $F 1+2(2.1 / 1.4 \mathrm{nmol} / \mathrm{L})$, and protein $\mathrm{C}(81 / 49 \%)$. In patients before resp. after kidney transplantation D-dimers (440/712 $\mathrm{mg} / \mathrm{L})$ and PAI-1 (35.2/61.1 $\mu \mathrm{g} / \mathrm{L})$ were increased as well besides t-PA. The values of patients after shunt preparation in comparison to those patients who had received a kidney transplant some time before elucidated that the latter patients showed a normalization of coagulatory activity. ( $\mathrm{p}<.05$; Mann Whitney test).

Depts. of nephrology and hematology/oncology ${ }^{2}$ of the Medical University at Luebeck, D-23538 Luebeck, Germany.

\section{1}

NEPHELOMETRIC DETERMINATION OF PLASMA D-DIMER. M. Kraus and E. Aillaud

A rapid assay for the determination of plasma $\mathrm{D}$-dimer has been developed using latex particle enhanced immunoassay. A monocional antibody (DD5) to human D-dimer was covalently coupled to activated aldehyde groups of shell/core polymethacrylamid/polystyrene particles $(280 \mathrm{~nm}$ diam.). The monodispersed particles were used for determination of human D-dimer in plasma on the BNA (Behring Nephelometer Analyzer, Behringwerke AG, Marburg, FRG): $50 \mu$ l of sample (1:5 diluted with $N$-diluent) were mixed with $50 \mu \mathrm{l}$ of the DD5-coated particles, $20 \mu \mathrm{l}$ of a supplement reagent and $140 \mu \mathrm{l}$ of $\mathrm{N}$-diluent. Light scattering was measured after $12 \mathrm{~min}$ incubation time and the D-dimer concentration was calculated from the reference curve. The reference curve was prepared automatically from a liquid Ddimer standard, covering the range from 62.5 to $2000 \mu \mathrm{g} / \mathrm{l}$ with respect to the concentration of the sample. For higher concentrations the sample could be diluted using $\mathrm{N}$-diluent.

The sensitivity limit is $62.5 \mu \mathrm{g} / \mathrm{D}$-Dimer. The normal range as determined preliminarily in citrated plasma from 104 blood donors ranged from smaller than $62.5 \mu \mathrm{g} / \mathrm{l}$ to $160 \mu \mathrm{g} / \mathrm{l}$. About $60 \%$ of the samples were below this limit. The $95 \%$-percentile was at $124 \mu \mathrm{g} / 1$. The intraassay coefficients of variation (CV) were $7 \%$ or better; the interassay CV were below $10 \%$. Upon addition of D-dimer up to $80,000 \mu \mathrm{g} / \mathrm{l}$ the results were still found above the highest reference point. No cross-reactivity with fibrinogen (up to 1000 $\mathrm{mg} / \mathrm{dl}$ ) or with fragment $\mathrm{D}$ (up to $300 \mathrm{mg} /$ ) was detected. No interferences could be observed due to rheumatoid factors or lipemic samples. Comparison with the Enzygnost D-Dimer micro revealed differences in the concentrations obtained, as often reported for heterogenous (ELISA) and homogenous assay comparison. Therefore, thorough examination of the clinical application of this more convenient assay has to be performed.
Ex vivo characterisation of compounds reactive to a fibrin specific antibody in plasma and effluent of CAPD patients.

M.Gödde ${ }^{\#}$, T.Sitter*, H.Hoffmann*, M.Spannagl ${ }^{\#}$ The balance of generation and proteolytic degradation of fibrin in the peritonal cavity plays an important role in the long term outcome of renal replacment therapy by CAPD. Increased procoagulant and/ or reduced fibrolytic activity are associated with fibrin induced adhesion phenomena and the risk of subsequent peritoneal fibrosis.

We have examined the level of fibrin monomer (FM) in plasma and peritoenal effluent of CAPD patients with and without bacterial peritonitis. FM was measured with an ELISA using a monoclonal antibody raised against a synthetic peptide identical to the $n$-terminal end of the $\alpha$-chain of human fibrin. Measurement was performed after incubation with chaotropic ions (NaSCN). Structural characterisation of the reactive componds in the patients plasma and effluent was performed after SDS electrophoresis by Westernblot using the same POD linked antibody as in the ELISA. In contrast to the ELISA in this system only one epitop is nessesary for binding the antibody.

All Patients showed six to ten fold higher levels of fibrinmonomer in effluent than in plasma. In patients with peritonitis we found lower concentration in effluent $(0,65 \pm 0,01 \mu \mathrm{g} / \mathrm{ml})$ as compared to patients $(16.9 \pm 1 \mu \mathrm{g} / \mathrm{ml})$ without peritonitis. The immunblotting showed two bands with a molecular weight of 270 and 340 dalton in plasma in both groups presumably representing high and low molecular FM. The peritonitis effluent contains two additionale bands with a molecular weight of 260 and 240 Dalton. The non peritonitis effluent exhibited 5 bands with a molecular weight ranging from 340 to 220 Dalton.

In conclusion we found lower levels of FM in plasma as compared to the peritoneal effluent, indicating reduced degregation. The additional bands in the effluent can be explained by a persistance of high molecular weight degregation products of fibrin in the peritoneal cavity.

Medizinische Klinik, Klinikum Innenstadt ${ }^{\#}$, Chirugische Klinik, Klinikum Großhadern*, Universität München, Ziemssenstr.1, D-80336 München 
MEASUREMENT OF FUNCTIONAL AND IMMUNOLOGIC LEVELS OF TISSUE FACTOR PATHWAY INHIBITOR. SOME METHODOLOGIC CONSIDERATIONS. W. Jeske, J. Fareed, D. Hoppensteadt, and J.M. Walenga. Loyola University Medical Center, Maywood, IL. 60153.

Tissue factor pathway inhibitor (TFPI) is a newly identified inhibitor of proteases generated during activation processes. Several functional methods based on a chromogenic substrate technique measuring the residual tissue thromboplastin/FVIla catalytic activity using excess FX and a chromogenic substrate for $\mathrm{Xa}$ (Sandset et al, Thromb Res 47:389-400, 1987; Zitoun et al, Thromb Res 72:269-274, 1993) have been published. Recently, a sandwich ELISA method (Imubind, American Diagnostica, Greenwich, CT) has become available. We have compared a modified functional method with the ELISA based antigen method in several groups of heparin treated patients and normal individuals. The functional TFPI method is sensitive to concentrations of TFPI from $2000 \mathrm{ng} / \mathrm{ml}$ to $125 \mathrm{ng} / \mathrm{ml}$ based on a rTFPI standard (Monsanto, St. Louis). The immunologic method is sensitive from 400 to $0 \mathrm{ng} / \mathrm{ml}$. A marked dichotomy has been observed between the two methods. The ratio between the TFPI antigen and functional levels varies widely in patient groups and during heparin treatment. We have supplemented this TFPI standard to buffer and normal human plasma and have measured the TFPI functionality before and after heat treatment at $56^{\circ} \mathrm{C}$ for 10 minutes. Heat treatment of plasma (buffer) after the supplementation of TFPI resulted in decrease TFPI functionality compared to the supplementation of TFPI after plasma (buffer) was heat treated. In contrast, the immunologic method does not require any heat treatment of the sample. Addition of exagenous TFPI to plasmas obtained from normal and the above patient plasmas demonstrated that the recovery of the functional TFPI was plasma dependent. Plasma proteins were found to markedly influence the TFPI functionality and antigenic quantitation. The TFPI standard supplemented in human pooled plasma gave an approximately $20 \%$ recovery in the immunologic assay and a $10 \%$ recovery in the functional assay. These results indicate that currently available methods may provide highly variable results on TFPI. Several matrix related effects should be taken into account for proper evaluation of TFPI.

\section{4}

CLINICAL EVALUATION OF A NEW ASSAY FOR DETERMINATION OF PROTEIN S ACTIVITY

C. Wagner*, J. Pabinger**, K. Fickenscher*

Protein $S$ acts as cofactor for activated Protein $C$ in the proteolytical inactivation of FVa and FVIIla. Only free Protein S exhibits anticoagulant activity. The new assay uses Russell's Viper Venom to activate FX and FV in the presence of activated Protein $C$. Since Protein $S$ deficient plasma substitutes all clotting factors except Protein $S$, the resulting proportion of inactivated $F V a$, and thus the prolongation of the clotting time, depends on the Protein $S$ activity. The assay can be run on all coagulometers, as well as on photometric instruments due to the addition of a chromogenic substrate.

The reproducibility of the new assay was evaluated by serial determination of a control plasma sample (target value $=86 \%$ ). The coefficient of variation was found to be $8.8 \%$ for intra-assay and $5.4 \%$ for inter-assay variability. In 61 healthy donors a mean Protein S (PS) activity of $81.6 \pm 16.6 \%$, range 51 $122 \%$, was found. Patients with hereditary PS deficiency $(\mathrm{n}=21)$ had a mean PS activity of $27.4 \pm 16.9 \%$, range $18-64 \%$. In 3 cases values around the lower border of the range of healthy donors were measured. In 54 patients on oral anticoagulants (OAC) mean PS activity was $53.9 \pm 14.4 \%$, range $17-84 \%$. The mean PS activity in hereditary PS deficient patients on OAC $(n=14)$ was $6.8+$ $5.0 \%$, range $0-17 \%$. There was only one patient within the range of the control group. In females on oral contraceptives $(\mathrm{n}=11)$ PS activity was substantially reduced $(\bar{x}=63 \pm 12 \%)$. Heparin treatment $(n=10)$ had no major influence on the PS activity.

Conclusions: With the new PS activity assay measurement of PS activity is possible on coagulometric and photometric instruments in routine setting. Most of the deficient patients were correctly identified, even those on OAC. However, if values between 50 and $65 \%$ are obtained in patients without $\mathrm{OAC}$, the additional determination of free and total PS antigen to exclude or ensure PS deficiency is recommended

* Behringwerke AG 35001 Marburg. Germany
** Universitätsklinik für Innere Medizin Abteilung für Hämostaseologie 1090 Wien, Austria
EFFECT OF APC ON THE APTT OF NORMAL BLOOD DONORS. M. Kraus and E. Aillaud

The addition of activated protein C (APC) to plasma leads to prolongation of the APTT via inactivation of FVIIIa and FVa. Occasionally, some cases have been reported in which this APC effect was partially suppressed. These patients suffered from systemic lupus erythematodes or from recurrent episodes of thrombotic events. This phenomenon was not due to autoantibodies to phospholipids or to coagulation factors, which quite frequently occur in such patients. Thus, some authors suggested the presence of an unknown inhibitor (Amer, L. et al. Thromb. Res. 57: 247258, 1990), protective effects of FIXa and vWF (Rick, M.E. et al.: J. Lab. Clin. Med. 115: 415-421, 1990) or the absence of an hitherto unknown cofactor of the protein $\mathrm{C} /$ protein $\mathrm{S}$ anticoagulant pathway (Dahlbäck et al.: Proc. Natl. Acad. Sci. USA, 90: 1004-1008, 1993).

Disturbances of the protein $\mathrm{C} /$ protein $\mathrm{S}$ anticoagulant pathway are believed to be a major cause for thrombotic disorders. Therefore, we were interested whether this so-called "APC-resistance" can also be found in apparently healthy persons. Thus, in citrated plasma of 136 blood donors the APTT has been determined using either $\mathrm{CaCl}_{2}$-solution or a $\mathrm{CaCl}_{2}$-solution containing APC for initiating the clotting reaction. With APC the clotting times reached ranged between $60.5 \mathrm{sec}$. and more than $325 \mathrm{sec}$. (3\% of samples), with a median of $167 \mathrm{sec}$. Different modes for calculating the "APC-resistance" were compared: total prolongation time, ratio of times with/without APC, \% of median prolongation time (\% MPT). Using different concentrations of APC and various APTT reagents, expression of the results in \% MPT allowed the best and most consistent comparison. $18 \%$ of the donors had a prolongation time of less than $70 \%$ of the median. The consequences of such an insufficient response to APC have to be established in follow-up studies.

Research Laboratories of Behringwerke AG, 35001 Marburg, FRG

A Novel ChromogeniC ASSAY FOR MBASURING APC
SENSITIVITY IN PLASMA
K.Váradi, B. Moritz, H. Lang, H.P. Schwarz

The plasma samples of some thrombophilic patients do not show prolonged APTT when APC is added (Dahlbäck et al: PNAS 90, 1004, 1993), i.e., the coagulation system is not sensitive to APC. The APTT is influenced by all coagulation factors of the intrinsic pathway. We investigated whether a test system based on the interaction of APC with one single factor - in this case FVIII - could improve the specific sensitivity of the APC response in patient plasma samples.

Test principle: The diluted plasma sample (as source of FVIII and APC cofactors) is incubated with FIXa, FX, phospholipids, $\mathrm{Ca}++$ and trace amounts of thrombin in the absence and presence of purified APC. The activated FX is measured by a chromogenic substrate, using Immunochrom ${ }^{(8)}$ FVIII:C. The amount of FXa generated is a function of the FVIIIa concentration. In the presence of APC, FVIIIa is partially inactivated by the APC/cofactor(s) complex(es). The ratio between the FXa activity without and with APC thus depends on the response of the plasma coagulation system to APC.

Plasma samples with "APC-resistance" - diagnosed with the Coatest APC Resistance- kit, (Chromogenix) had significantly lower ratios than plasma samples of healthy donors.

Although the APC sensitivity test described here is based on a FVIII activity assay, the results are not influenced by the actual FVIII activity as long as the plasma FVIII levels are in the normal range (50-150\%). The influence of other coagulation factors is also negligible. Our FVII-assay system is not influenced by heparin either. Therefore, this APC sensitivity test can be performed not only in untreated patients, but also in patients already on anticoagulant treatment (heparin and coumarin derivatives). 
POSSIBLE INTERACTION OF APC-RESPONSE ON ClotTING PROTEIN S ASSAYS. B. Moritz ${ }^{1}$, W.M. Halbmayer ${ }^{2}$, H. Lang ${ }^{1}$, M. Fischer ${ }^{2}$

The Protein C / Cofactor system plays a major role in disease with thrombotic implications. In addition to Protein $\mathrm{C}$ and Protein $\mathrm{S}$ deficiency, poor APC-response seems to be a cause of thrombotic events (Dahlbäck et al., 1993).

The aPTT assay was performed in the absence and in the presence of activated Protein C (APC) and APC-response was expressed as ratio of aPTT/APC-aPTT. The normal range was established by calculating the mean $\pm 2 S D$ of the APC-response ratio in 50 persons with normal coagulation; poor APC-response corresponds to values $<$ (mean - 2SD). The following two assay systems were established: Method A: Coatest APC Resistance (Chromogenix), nommal range (mean $\pm 2 S D$ ): 2.2 - 3.8 (Halbmayer et al., 1993); Method B: aPTT-reagent based on kaolin/ sulfatides / highly purified phospholipids \pm APC $(0.3 \mathrm{U} / \mathrm{ml}$ final concentration), nomal range: 1.8 - 4.4.

Fiftyfive patients with thrombotic implications (arterial or venous) (without oral anticoagulation therapy) were investigated. Fifteen patients showed poor APC-response: 6 in both methods, 1 in method A, 8 in method B. One patient with known Protein $S$ Deficiency (Type I) was excluded from further considerations.

Protein S was quantitied using antigen assays (total Protein S): (1) ELISA (Boehringer Mannheim), (2) EID (Immuno) and functional assays: (3) using a Protein S-sensitive bovine thromboplastin (IL; Preda et al., 1990), (4) inhibition of F.Va in the presence of APC (Stago; Wolf et al., 1990), and (5) F.Xa one-stage clotting assay (Comp et al. 1984). The nomal range was taken from the manufacturer's instructions or from our own laboratory results. Methods 1,3 and 5 were performed in all plasma samples, methods 2 and 4 only in those with poor APC-response.

In the group with poor APC-response, the protein $S$ levels measured with both antigen assays as well as with the F.Xa-based functional method (5) were all within the normal range. With the two other functional assays, some abnomal low values were found: method (3): $6 / 14$ (40\%); method (4): $2 / 14(15 \%)$.

From our data, we conclude that APC-response phenomenon influences the assays based on bovine thromboplastin and on the inhibition of F.Va more than it influences the F.Xa one-stage clotting assay.

IImmuno AG, Industriestr. 67, A-1220 Vienna / 2Municipal Hospital Vienna-Lainz, Central Laboratory, Wolkersbergenstr. 1, A-1130 Vienna

\section{DETERMINATION OF PROTEIN C IN PROTEIN C-CONCENTRATES} J.-D. Schreiber, H. Igel, H. Lang

Amidolysis of chromogenic substrates after activation by PROTAC is an established and reliable methodology for the determination of Protein C in plasma samples. Special advantages over clotting methods are increased sensitivity and better reproducibility.

The WHO standard for $\mathrm{PC}$ is a calibrated pooled plasma. In order to transform its potency to a system containing purified coagulation factors it is necessary to establish an equivalent concentrate standard. According to the "Principle of Equivalence" this standard should then be used for assessment of the potency of concentrates.

During calibration of the standard the following criteria have to be met to ascertain a valid transformation of the potency of the WHO plasma standard to the concentrate standard:

1. comparable matrix ( i.e.: predilution in PC-deficient plasma to a concentration of $1.0 \mathrm{IE} / \mathrm{ml}$ )

2. linearity of the serial dilutions

3. linearity of the calibration curve

4. parallelism between serial dilutions of standard and samples

5. correct values of previously calibrated plasma controls

As it could be demonstrated that all these criteria were met, the assignment of a potency to the PC concentrate standard in a PC-deficient plasma predilution system seems to be possible.

Assessment of potency of concentrates is to be made preferably in a buffer predilution system, since this is more appropriate for working with purified preparations. A series of tests was performed using the PC concentrate standard as reference and assaying the potency of several batches of PC concentrates in both a PC-deficient plasma and a buffer predilution system. Again in each predilution system the above mentioned criteria were fulfilled.

We propose as appropriate method for PC determination in PC concentrates:

- Calibration of a concentrate standard in PC-deficient plasma predilution against WHO.

- Use of this standard for determination of potency of $P C$-concentrates in buffer predilution.
INCIDENCE OF RESISTANCE TO ACTIVATED PROTEIN C PRELIMINARY RESULTS FROM A STUDY IN HEALTHY BLOOD DONORS

M. Steiner, C. Burstein, F. Rabe\# , O. Anders ${ }^{*}$, K. Stange ${ }^{*}$, B. Ernst

Diminished anticoagulant response in plasma to activated protein $C$ (APC) has recently been suggested to predispose to thromboembolic events. Although a growing number of inherited thrombophilia has been reported to be associated with APC resistance only limited data are available concerning the incidence in a general population. Therefore, we have screened healthy blood donors for the presence of APC resistance. 94 blood donors were included (41 female, 53 male, aged between 19 and 57 years). APC resistance was investigated in citrate-anticoagulated plasma using COATEST APCResistance (Chromogenix) and the coagulation analyzer ACL 3000 (Instrumentation Laboratory). The ratio between aPTT prolonged by addition of APC and pure aPTT was calculated. The mean ratio was found to be 3.04 with a standard deviation of 0.74 . Individual ratio ranged from 1.68 up to 5.08 . Based upon these results the normal range would be 1.56 to 4.52 and no pathological case was recorded in this investigation. However, a ratio below 2.0 has been suggested to be suspect for poor anticoagulant response to APC. 8 out of 94 blood donors $(8.5 \%$ ) fulfil this criterion. These blood donors should be reinvestigated and additional family studies should contribute to exclude a hereditary defect. Further studies are ongoing to assess the incidence of APC resistance in the general population.

University of Rostock, Institute of Clinical Chemistry \& Pathobiochemistry, Department of Internal Medicine ${ }^{*}$, Heydemann-Str. 6 , 18055 Rostock, Germany, Chromogenix AB\#, Mölndal, Sweden

\section{0}

RESISTANCE TO ACTIVATED PROTEIN C IN PATIENTS WITH DEEP VEIN THROMBOSIS

C. Burstein,M. Steiner,B. Ernst,F. Rabe ${ }^{\#}$, B. Krammer ${ }^{*}$,O. Anders ${ }^{*}$

Several inherited and acquired abnormalitites within the coagulation and fibrinolysis systems are generally accepted to be associated with the occurrence of deep vein thrombosis (e.g. hypofibrinolysis, coagulation inhibitor deficiency). However, many patients suspicious for thrombophilia remain without any detectable biochemical defect predisposing to thromboembolic events. Recently, reduced anticoagulant response in plasma to activated protein C (APC) has been shown to be present in patients who had suffered spontaneous deep vein thrombosis (DVT). Therefore, we have integrated the investigation of APC resistance in our thrombophilia screening program. 12 patients who had suffered DVT and 18 newly hospitalized patients with DVT were investigated. APC resistance was measured using COATEST APC-Resistance (Chromogenix) and the coagulation analyzer ACL 3000 (Instrumentation Laboratory). The ratio between aPTT proionged by addition of APC and pure aPTT was calculated. Relative to controls, patients with history of DVT demonstrated reduced mean ratio $(2.2 \pm 0.53$ vs $3.04 \pm 0.74)$. 4 patients $(33 \%)$ revealed ratio less than $2.0(1.3-1.8)$ which is suspect for APC resistance. 18 patients with ongoing thrombosis were found to have decreased mean ratio $(2.3 \pm 0.64) .7$ patients ( $39 \%$ ) demonstrated values below $2.0(1.6-1.9)$. The data suggest that in a significant number of patients with DVT the presence of APC resistance could contribute to thrombophilia and thrombogenesis.

University of Rostock, Institute of Clinical Chemistry \& Pathobiochemistry, Department of Internal Medicine ${ }^{*}$, Heydemann-Str. 6, 18055 Rostock, Germany, Chromogenix AB\# , Mölndal, Sweden 


\section{APC RESISTANCE \\ IN PATIENTS WITH THROMBOEMBOLIC DISEASES \\ S. Ehrenforth, E. Aygören-Pürsün, B. Zwinge, I. Scharrer}

Introduction: Recently a new mechanism for thrombophilia has been described in which the anticoagulant response to activated protein C (APC) is impaired due to a deficieny of a novel PC cofactor. Whether the APC resistance is resulting in an increased risk of thromboembolism respectively represents a hereditary disorder which contribute to the pathogenesis of familial thrombophilia is at present a matter of discussion.

Patients and Methods: A total of 137 patients which are suffering from thromboembolic events were investigated in regard to their response towards addition of APC, using the activated partial thromboplastin time - based assay "Coatest APC resistance" (Haemochrom, Essen, Germany). In case of APC resistance, addition of APC to the plasma did not result in a normal antiooagulant response as measured by prolongation of the APTT. The APC response was expressed as the ratio between the clotting times obtained in the presence and absence of APC. 80 healthy persons served as controls (APC ratio-95\% percentile: female: $1.93-3.84$, male: $1.97-4.56$ )

Results: An abnormally APC ratio $(<2)$ was detected in $17 / 137$ pts. $(15.6 \%)$. In one family the combined occurrence of familial sphaerocytosis, recurrent deep vein thrombosis, pulmonary embolism, familial APC resistance and functional protein $S$ deficiency is striking. The female propositus and her both daughters had had deep vein thrombosis and pulmonary embolism at an early age ( $29 \mathrm{ys}, 17 \mathrm{ys}$, respectively $28 \mathrm{ys}$ ) after short-term oral anticonceptive therapy. In her grand-daughter no thromboembolism has occurred until the actual age of 9 ys. The propositus and her two daughters demonstrated a significant APC-resistance in the APTT-based assay (APC ratio 1.12, 1.17 resp. 1.18), whereas in the grand-daughter an APC ratio of 1.86 was determined. An apparent functional PS deficiency was found in all of them (PS activities between 26 and $42 \%$ ). In the propositus' husband a APC ratio of 1.96 and a PS activity of $63 \%$ could be determined. Summarizing, a hereditary APC resistance is presumed in this family which led to familial thromboembolism.

Dept. of Angiology, University Hospital, 60590 Frankfurt, Germany

DEEP VENOUS THROMBOSIS AND POOR ANTICOAGULANT RESPONSE TO ACTIVATED PROTEIN C (APC)

U.T. Seyfert, M. Kuemmel and E. Wenzel

A new hereditary disorder has been described by $B$. Dahlbaeck 1993 which may be an important underlying cause for thrombophilia. This disorder is characterized by an abnormally low anticoagulant response in plasma on addition of activated protein $c$ (APC).

Patients and methods: 100 normals and patients with an history of deep venous thrombosis (blood sampling 6-18 months after an thromboembolic episode) were investigated. The lab panel included inherited deficiencies of anticoagulant proteins (AT III, Protein C, Protein S, Heparincofactor II, Plasminogen, Tissue factor pathway inhibitor), thrombin generation rate (TAT, Dimers, F1,2), Antiphospholipid antibodies and fibrinolytic response before and after venous occlussion (PAP, tPA, PAI). APC was measured by a commercially available kit (COATEST APC Resistance, Chromogenix Essen).

Results: A normal ratio of APC resistance is indicated when the ratio is $>2(2.9+-0.9)$. In a cohort of 100 patients with deep venous thrombosis $14 / 100$ patients had an APC ratio below 2.0 (ratio $1.8+-0.8), 86 / 100$ patients a ratio $>2(2.96+-$ 1.6). The two-tailed $p$ value is $<0.0001$. We observed no significant differences in fibrinolytic response and behaviour of PAP betweeen both groups. Conclusion: Poor anticoagulant response to activated protein $c$ may constitute an important risk factor for hypercoagulability and thrombophilia.

Address: Klin. Haemostaseologie, Unikliniken, Haus 75 , D- 66421 Homburg
SENSITIVITY OF THE RECOMBINANT THROMBOPLASTIN INNOVIN'M FOR THE DETECTION OF A REDUCTION OF THE ACTIVITY OF THE EXTRINSIC COAGULATION FACTORS: COMPARISON WITH CONVENTIONAL THROMBOPLASTINS K.-J. Hartung, D. Kunz, G. Lutze

The reduction of the activity of the extrinsic coagulation factors (II, V, VII and $\mathrm{X}$ ) is detected with variable sensitivity using commercially available thromboplastin reagents. Therefore, it is necessary to be aware of the responsiveness to detect a depletion of the factors in order to be able to assess the capabilities and limitation of a thromboplastin time assay. We have evaluated the sensitivity of the recombinant thromboplastin Innovin ${ }^{\text {TM }}$ (Baxter Diagnostics Inc.) and of the conventional tissue thromboplastins Immunoplastin (B) HIS (Immuno AG, Wien), Thromborel(B) S (Behringwerke AG, Marburg) and Neoplastin Plus (Boehringer Mannheim $\mathrm{GmbH}$ ) and compared the results. Based upon our findings we conclude:

1. Innovin detects a reduction of the activity of each of the 4 factors of the extrinsic pathway with better sensitivity than the conventional thromboplastins. This was found for artificially prepared mixtures of plasma with deficient plasma and also for patient plasmas.

2. The sensitivity of the conventional tissue thromboplastins is less but very similar among each other.

3. For all thromboplastins not only the activity of the investigated factor in the test plasma has to be considered alone but also the relative activities of the other extrinsic factors.

Address: Otto-von-Guericke-Universität, Medizinische Fakultät, Inst. f. Klinische Chemie, Leipziger Str. 44, 39120 Magdeburg

\section{4}

\section{EVALUATION OF A NEW CHROMOGENIC PROTHROMBIN TIME} (NYCOTESTTM CHROM) ASSAY

L. Volbracht ${ }^{1}$, A. Krützfeldt' ${ }^{1}$, R.S. Ross ${ }^{1}$, Th. Eller ${ }^{1}$ K. Bürvenich ${ }^{2}$, D. Paar?

In the chromogenic prothrombin time (PT) assay coagulation activity is reflected by the time to reach a certain change in absorbance (fixed absorbance method). We evaluated a new chromogenic prothrombin time assay (Nycotest Chrom, Immuno GmbH, Heidelberg, Germany) in comparison to two coagulometric PT assays and a second chromogenic PT assay.

Materials and Methods: Photometric measurements using Nycotest Chrom and Chromoquick (Behringwerke AG, Marburg. Germany) were done on Chromo Time System (Behringwerke AG). Coagulometric determinations (Coagulometer KC40/KC10, Amelung, Lemgo, Germany) were performed using Immunoplastin HIS (Immuno GmbH) and Thromborel S (Behringwerke AG). Imprecision was determined using control plasma (Control Plasma Normal, Control Plasma AK-R, Immuno GmbH) and patient samples.

Results: Imprecision (PT range 98-18\%): intra assay ( $=20, \mathrm{CV}): 1.7$ $3.6 \%$ for Nycotest Chrom compared to $2.1-4.9 \%$ (Chromoquick), 1.6 $5.6 \%$ (Thromborel S), and $2.8-4.8 \%$ (Immunoplastin HIS); Inter assay (2 series of 10 days, $C V): 3.8-8.8 \%$ for Nycotest Chrom compared to 4.6 9.5\% (Chromoquick), 2.4 - 3.8\% (Thromborel S) and $2.1-5.3 \%$ (Immunoplastin HIS). Comparative studies covering the PT range of 119 $13 \%$ showed a good correlation $(r>0.96)$ between Nycotest Chrom and the other PT assays tested. Concerning the therapeutic range in patients receiving oral anticoagulant therapy we obtained the same classification for Nycotest Chrom compared to Immunoplastin HIS or Thromborel $\mathbf{S}$, respectively, in more than $90 \%$ of the cases; between Nycotest Chrom and Chromoquick there was an agreement of $70 \%$.

Conclusion: The chromogenic prothrombin time assay Nycotest Chrom shows an adequate reproducibility and good correlation to the other PT assays investigated. Therefore, it represents an alternative to coagulometric prothrombin time determination.

1 Division of Clinical Chemistry and Laboratory Diagnostics, Department of Internal Medicine, University of Essen, Hufelandstr. 55 45122 Essen, Germany

2 Immuno GmbH, Heidelberg, Germany 


\section{LOT TO LOT CONSISTENCY OF A PT REAGENT BASED ON RECOMBINANT TISSUE FACTOR AND SYNTHETIC PHOSPHOLIPIDS}

H.-J. Kolde, B. Denzler, P. Hawkins*, H. Pelzer*

Baxter Diagnostics Unterschleißheim and *Miami

The introduction of recombinant human tissue factor and synthetic phospholipids should allow a better standardization of manufacturing of a thromboplastin (Innovin "'", "I"). We investigated this assumption by comparing 12 different lots of I on three different types of coagulometers based on turbidimetry, nephelometry or mechanical endpoint detection. The samples were a normal and an abnormal control plasma, three pools of patients with liver disease and two levels of oral anticoagulation (OA) and a normal plasma pool. The ranges of $\mathrm{cv} \%$ values of the clotting times in sec. were $1.5-6.3 \%, 2.1-5.6 \%$ and $2.6-8.3 \%$ respectively on the three instrument types. For the two OA plasmas the precision in the INR were in the range of $2.1-4.3$. Thus they are in the same order or magnitude as the determination of the ISI or the within run precision. The absolute values of the INR for the two plasmas were $3.20,3.10$ and 3.29 (sample 1) or 2.18 2.29 and 2.21 (sample 2) respectively on the three instruments. The intra assay precision of the various lots was very similar. The ISI values standardized against BCT by an external reference institution - ranged from 3.3 to $4.1 \%$ c.v. for the three instrument types in the first 14 lots. In an additional investigation we compared 20 normals and 40 patients on stable anticoagulant treatment using three consecutive lots of Innovin on an Electra 900. The correlation coefficients were $\geq 0.998$ when the data were analyzed in INR. The regression lines were not significantly different from $y=x$. We are not aware from published batch consistency data of conventional thromboplastins in the literature and thus a direct comparison is not possible. At least these data show that the use of recombinant human tissue factor and synthetic phospholipids leads to a well standardized production procedure with lots with almost identical properties.

Address: Baxter Diagnostics, Scientific Support Hemostasis, Edisonstraße 3, D-85716 Unterschleißheim and ${ }^{*}$ Haemostasis R \& D, Miami, FI, USA

Clinical experience with heparin-insensitive thromboplastin reagents based on Thromborel $\mathbf{S}^{\mathrm{TM}}$

M. Heins', W. Withold', R. Behnke², W. Rick

'Institut für Klinische Chemie und Laboratoriumsdiagnostik der Universität Düsseldorf, ${ }^{2}$ Abteilung für Kardiologie der Universität Düsseldorf

The important indications for the determination of the thromboplastin time (Quick) are the controlling of patients under oral therapy with cumarine derivates as well as the detection of clotting factor deficiency in liver disease and vitamin $K$ deficiency Whereas a high dosage heparin therapy ( 32500 to $45000 \mathrm{IU} / 24 \mathrm{~h}$ ) also yielded a decrease of the Quick value (in percent of normal activation), the interpretation of the Quick value for these situation is limited. Therefore the availability of reagents for the determination of the thromboplastin time beeing unsensitive against heparin is desirable.

Thromborel S (Behringwerke Marburg), a lyophilisized human placenta thromboplastin was reconstituted with aqua bidest. as well as with two heparin-neutralizing polybrene-buffers $(\mathrm{ZO}, \mathrm{ZP})$ in order to find out the influence of the different reconstitution mediums onto the quick.

A randomized selected collective of 85 patients with nornal plasma coagulation (quick $>70 \%, 60$ patients with a quick from 31 to $69 \%$ and 66 in the therapeutic range ( 62 patients with a quick from 15 to $30 \%$ and 4 patients with a quick below $15 \%$ ) were compared with the three reconstitution mediums at the coagulation analyser $\mathrm{KC} 10$ (Amelung, Lemgo)

For those 127 patients without heparin therapy comparison according Passing and Bablok showed following results: Thromborel - Aqua bidest. $(\mathrm{x})$ versus $\mathrm{ZO}(\mathrm{y})$ $y[\%]=1.0000 x[\%]-1.0000, r=0.993$; Thromborel - Aqua. bidest. $(x)$ versus ZP (y): $y[\%]=0.9655 x[\%]+2.5172, \mathrm{r}=0.996$

Using buffer $Z 0$ the other 84 patients under heparin therapy showed similar results as after reconstitution with aqua bidest: $y[\%]=1.0000 \times[\%]+2.0000, r=0.955$. The better heparin-neutralisation of buffer ZP - compared with aqua bidest.- could be seen in the partially higher values: $y[\%]=0.9574 x[\%]+5.7979, \mathrm{r}=0.943$. In 6 of 84 patients $(7 \%)$ we observed an increase of the Quick value from 20 to $72 \%$ after reconstitution with $\mathrm{ZO}$ in relation to aqua bidest; in 9 from 84 patients $(11 \%$ ) an increase from 22 to $88 \%$ was found after reconstitution with $\mathrm{ZP}$ in relation to aqua bidest..
Comparison between a recombinant thromboplastin and human placenta thromboplastin

M. Heins, W. Withold, E. Schnaith, H. Dauwitz, S. Schmitz, H. Reinauer Institut für Klinische Chemie, Heinrich-Heine-Universität Düsseldorf

A new thromboplastin based on a recombinant manufactured tissue factor and synthetic phospholipids $($ RTP $=$ Innovin, Baxter) was compared with a human placenta thromboplastin (HPT $=$ Thromborel $\mathrm{S}$, Behringwerke) in this study.

Differences were observed in the heparin sensitivity. In an in-vitro experiment pool plasma from 10 healthy persons was mixed with heparin (Liquemin, Roche). The Quick value of the HPT decreased to the lower limit of the reference interval $(70-120 \%)$ after adding of $1.2 \mathrm{IU} / \mathrm{ml}$ heparin, while this effect was observed in the RTP after $3.2 \mathrm{IU} / \mathrm{ml}$ Heparin (reference interval $80-140 \%$ ). So the patients were divided in two collectives, one without and one with heparin therapy.

129 patients without heparin therapy were analysed, 39 with a quick (HPT) from $70-117 \%$ [RTP: $79-135 \%$, 19 with a quick (HPT) from 31 - $69 \%$ [RTP: $28-80 \%$ ], 66 with a quick from $15-29 \%$ [RTP: 7 - $31 \%$ ] and 5 with a quick from $10-14 \%$ [RTP: 9 - $15 \%$. For these patients the comparison according to Passing and Bablok showed the following analyses: (Innovin $(x)$ versus Thromborel $(y): y[\%]=0.816 x[\%]+6.149$, $r=0.986$ ).

In 47 patients with heparin therapy, 14 of them with additional phenprocoumon therapy, a higher recovery with HPT could be seen (Innovin ( $\mathrm{x}$ ) versus Thromborel $(y)$ : $y[\%]=0.800 \times[\%]-4.200, r=0.929)$.

For testing the clotting factor sensitivity pool plasma was mixed with factor deficiency plasma. In factor $\mathrm{X}$ and II RTP showed a better factor sensitivity below an activity of $5 \%$. A deficiency of factor $V$ could be detected with higher sensitivity using HPT. RTP was highly sensitivy concerning the detection of factor VIl deficiency below an activity of $10 \%$. Particularly sensitive was the RTP in the detection of F VII deficiency, where a better sensitivity could be seen. A corresponding result was found in the factor analysis of 24 patients with a quick below $30 \%$.

\section{METHODS FOR DETERGENT-FREE RELIPIDATION OF TISSUE FACTOR AND OTHER MEMBRANE PROTEINS}

\section{Norbert F. Zander and Karl Fickenscher}

After purification of membrane proteins, restoring activity in many cases requires relipidation, i.e. integration in suitable lipid vesicles.

Standard methods of relipidation take advantage of detergents. Membrane proteins are mixed with phospholipids in the presence of a suitable detergent, followed by detergent removal, either by means of dialysis, dilution or by detergent-binding beads.

We report two methods to relipidate membrane proteins without the need of adding detergents. Recombinant human Tissue Factor (huTF) was expressed in E.coli cells and purified by immunoaffinity chromatogaphy. Effective relipidation resulting in an active protein/phospholipid complex could be achieved:

(a) by mixing buTF with phospholipid, adjusting the $\mathrm{pH}$ to a value below 3 , neutralization, and buffer addition, or,

(b) by mixing buTF with phospholipid, heating the mixture to a temperature above $80^{\circ} \mathrm{C}$, cooling, and buffer addition.

Using Prothrombin Time (PT) reagents produced with huTF relipidated as described above, a plasma pool of normal donors had a PT of ca. 11 seconds. The ISI of the reagent was 1.0 .

Behringwerke AG, 35001 Marburg, Germany. 
ACCURACY OF THE DETERMINATION OF THE INTERNATIONAL SENSITIVITY INDEX (ISI) USING PRIMARY AND SECONDARY STANDARDS

E. Gurr, D. Baykut

Results in thromboplastin time determinations are recommended to be reported in terms of INR with respect to oral anticoagulation control. The ISI value of a definite reagent varies with instruments used for the determination. ISI values are not always declared by the manufactures for all instruments. Reference thromboplastins (BCT) are recommended for the determination of ISI values, but they are expensive and often not available in time. To estimate an ISI value the procedure for the determination, reported by Kirkwood (1993) was performed for the systems Innovin/CA 5000 and Thromborel S/CA 5000 , using Thromborel S/KC 10 and Innovin/KC 10 as secondary standard systems. The primary standard systems used for control was BCT $253 / \mathrm{KC10}$. The differences between the ISI values obtained by the use of the primary and the secondary standards were found below $5 \%$, demonstrating that the use of a secondary standard system allows the estimation and/or control of an ISI value. However the influence of different types of regression line calculation (Passing/Bablok vs. "Standardisierte Hauptkomponentenanalyse") was found above 5\%.

Zentrallaboratorium

Zentralkrankenhaus "Links der Weser"

Senator-WeBling-Str. 1

28277 Bremen

A COMPARISON OF DIFFERENT THROMBOPLASTINS FOR THE DETERMINATION OF THE EXTRINSIC COAGULATION

FACTORS

H.Reuter, V.Tilsner.

University Hospital Hamburg, Dep.Haemostaseology,

With the availability of thromboplastin reagents based on recombinant human tissue factor we were interested to investigate the performance of such a preparation in comparison to the established PT reagents based upon tissue extracts. For this purpose we used commercial thromboplastins from either rabbit brain, human placenta and recombinant buman tissue factor / synthetic phospholipids (Innovin "Bu ,Baxter Diagnostics, "I") and have evaluated their performance in patients with liver disease, liver transplantation and in patients with heparin therapy. In specific factor assays the slopes of the calibration curves showed significant differences. The most pronounced increase of responsiveness was found for factor VII in which the recombinant reagent was clearly superior over the two tissue thromboplastins. Not unexspectedly the correlation of the activity of the extrinsic factors and the prothrombin time was more plausible for the recombinant preparation in patients on heparin treatment. This could be a consequence of the addition of a heparin neutralising agent in " 1 ". In other patients, however, a similiar trend was found as well. Especially in the group of patients with liver transplantation the closest correlation between the activity of the extrinsic facors, in particular for factor $\mathrm{V}$, and the prothrombin time was observed for "I", followed by the placental and the rabbit brain reagent. Based upon our findings we conclude that the closer agreement of PT results of the recombinant preparation with factor assays is related to its higher sensitivity and the fact, that this reagent is not contaminated with residual coagulation factors. The heparin insensitivity is an additional benefit in various groups of patients. The use of recombinant tissue factor in the production of thromboplastins may be a gain in the quality of laboratory results, may reduce the number of unnecessary factor assays and prevents the potential risks associated with the handling of human or animal tissues.

\section{1}

DIAGNOSTIC EQUIVALENCE OF MAMMALIAN AND RECOMBINANT TISSUE FACTOR BASED THROMBOPLASTIN REAGENTS. D. Hoppensteadt ${ }^{*} \mathrm{C}$. Blakemore, "D. Callas," S. Moran," J. Fareed R.L. Bick, and E.W. Bermes." Loyola University Medical Center, Maywood, IL.

Most commercially available PT reagents (INR 1.5-2.6) consist of crude extracts of either mammalian or human tissue as the source of tissue factor (TF). Recently, recombinant human (TF) expressed in E. Coli has been used to develop PT reagents with claimed ISI value between 1.0 and 1.10. We have compared several mammalian thromboplastins with two commercial recombinant TF preparations, Innovi ${ }^{R}$ (Baxter Dade; Miami, FL) and Recombiplastin ${ }^{\mathrm{R}}$ (Ortho Diagnostics; Raritan, NJ). In the PT assay, both r-TF's showed higher sensitivity than the natural TF's when normal human plasma was diluted to give $100-6.25 \%$ of all factors. In a second study the sensitivities to factors I, II, V, VII, IX, X, XI, XII, prekallikrein and high molecular weight kininogen were tested using dilutions of factor deficient plasmas. Innovin ${ }^{\wedge}$ and Recombiplastin ${ }^{\wedge}$ were more sensitive to the lower concentration of each of these factors than the mammalian preparations in the PT assay. In addition. r-TF preparations were found to be more sensitive to plasmas from oral anticoagulated patients than the mammalian thromboplastins. A PT of 1314 seconds with mammalian thromboplastin gave values between $16-20$ seconds with the r-TF. Furthermore, no significant differences were noted among the reagents when the results were compared in terms of INR values of $\leq 3.0$, however, at INR values $>3.0$, differences were noted. Interestingly, TF antigen levels as measured by a newly developed Elisa assay (American Diagnostica) were found to markedly differ in the two recombinant preparations. Of the mammalian reagents tested, monkey and human brain thromboplastins showed TF antigen levels, whereas rabbit and human placenta did not. Our studies demonstrate that because of the origin and composition, the values obtained with the newly introduced recombinant thromboplastins can not be directly substituted for the values obtained with the currently available standardized prothrombin reagents in PT and factor assays.

A NETWORK COMPATIBLE COMPUTER PROGRAM FOR RECORDING AND EVALUATION OF PATIENT DATA IN A HAEMOSTASEOLOGY LABORATORY.

C. Stanescu, Th. Vigh, E. Dingeldein and I. Scharrer

Computer based management of patient data has become an invaluable help in clinical settings where bandling large amounts of patient related informations and lab results is required.

We have developed a computer program for a haemostaseologic outpatient clinic which we named AN GIO. The program has been in use for over one year and was used to process information about 3500 patients including approximately 63000 patient related test results.

ANGIO was designed to file patient related data such as the patients personal bealth history, his family bealth history, the development of diseases, medication and treatment plans, lab results, as well as information from other departments and primary physicians. ANGIO is also capable to evaluate these data for scientific purposes, using various methods of statistical analysis.

If run on a network system like the Novell network ANGIO allows convenient entry and retrieval of data from all participating terminals. Text blocks and tables and lab results can be assembled individually, allowing customized printouts.

Although ANGIO was designed for the specific needs in our outpatient haemostaseology lab it can be easily adapted to meet the requirements of other clinical settings where large amounts of patient data are recorded and processed.

In summary, we present a network compatible computer program which allows convenient entry and processing of patient related data from many participating terminals, thus providing an invaluable tool for managing the ever increasing load of informations in the daily routine of outpatient laboratories or other clinical settings.

Present Address: Dept. of Angiology, Center of Internal Medicine, University Hospital, Frankfurt/Main, FRG 
CHARACTERIZATION OF A SUBGROUP OF PATIENTS WITH MILD HAEMOPHILIA A AND DISCREPANCY IN ONE STAGE- AND CHROMOGENIC FACTOR VIII ASSAYS

J. Oldenburg, R. Schwaab, J. Faber, H.-H. Brackmann

124 Patients with mild and moderate haemophilia were screened for mutation in the factor VIII gene. Four patients out of three families showed a mutation at the beginning of exon 14 at amino acid 720 (GAG(Glu)720 ---> AAG(Lys)) and 2 patients out of one family showed a mutation at the end of exon 14 at amino acid 1689 (CGC(Arg)1689--)> CAC(His)).

In all of these patients we found a reduced factor VIII activity of $10-30 \%$ with the one stage assay but normal factor VIII activities with the chromogenic factor VIII assay. This discrepancy was stable with restudy on multiple occasions with three different chromogenic assays (Baxter, Immuno, Chromogenics) and two different one stage assays (natural factor VIII deficient haemophilic plasma, Merz \& Dade). All patients showed higher levels of factor VIII antigen in comparison with the procoagulant activity (one stage method). The clinical picture correlates with the factor VIII activity measured with the one stage method.

Since one mutation is at the $5^{\prime}$ end of the B-domain very near to a thrombin cleavage site and one mutation is within the thrombin cleavage site at the 3 'end of the B-domain, the B-domain may be uncompleted spliced. This data suggest, that the higher factor VIII activities measured with chromogenic assays could be linked to possible molecule activation or decreased inactivation associated with modifications in the thrombin, $X_{a}$ or activated protein $C$ cleavage sites.

\section{4}

APPLICATION OF A NOVEL ENZYME-LINKED IMMUNOSORBENT ASSAY (ELISA) METHOD TO STUDY THE PHARMACOKINETICS OF RECOMBINANT HIRUDIN. L.Iver, J.Amiral' and J.Fareed. Depts. of Pharmacology and Pathology, Loyola University Chicago, USA and Serbio Research Labs. France.

In recent times, there has been a major interest in the development of recombinant hirudins ( $\mathrm{rHs}$ ) as anticaagulant and antithrombotic agents in various clinical conditions. These proteins are recombinant analogues of native hirudin, obtained from the medicinal leech, Hirudo medicinalis. In order to achieve a wider acceptance of rits as clinically useful drugs, it is crucial to establish and validate a reproducible assay method for $\mathrm{rHs}$. Most earlier assay methods employed the use of indirect pharmacodynamic measurements such as activated partial thromboplastin time, prothrombin time, thrombin time and a chromogenic thrombin substrate method. We have employed a novel immunological method to determine $\mathrm{rH}$ concentrations in biological fluids such as plasma and urine, directly. The method is based on a competitive ELISA technique where the $\mathrm{H}$ in test samples competes with antigen in precoated microplates to bind to polyclonal anti-hirudin antibodies raised in rabbits. We have used this assay method to determine true pharmacokinetics of $\mathrm{rH}$ in animal models such as rabbits, dogs and rats. New Zealand White rabbits $(n=5$ ) were injected with $25(i, v)$ and 375 (s.c.) $\mu \mathrm{g} / \mathrm{kg}$ doses of $\mathrm{rH}$ variant 2 ( $\mathrm{rHV} 2$ ). Mongrel dogs $(n=6)$ were injected with i.v. and s.c. doses of $0.25,0.5$ and $1.0 \mathrm{mg} / \mathrm{kg}$ of rHV2 whereas Sprague-Dawley rats $(n=24)$ were injected with $0.1,0.4 \& 1.0$ (i.v.) and $0.1,0.5$ and $1.0 \mathrm{mg} / \mathrm{kg}$ (s.c.) doses of rHV2. In all three models, rHV2 was rapidly distributed and eliminated with terminal elimination half-lives of less than an hour after i.v. administration. S.C. administration exhibited a well-defined absorptive phase followed by a peak at 2 to $\mathbf{4}$ hours and a rapid elimination phase. Clearance rates approximated about 250 and $75 \mathrm{mls} / \mathrm{kg} / \mathrm{hr}$ in dogs and rabbits. respectively. This immunoassay offers one of the first direct methods to measure absolute concentrations of $\mathrm{rHs}$ in biological fluids enabling the distinction of pharmacokinetics from pharmacodynamics of rHs. The ELISA method is simple, rapid, sensitive and reproducible (C.V. $=4.5 \%$ ) and can easily be adapted for pharmacokinetic \& pharmacodynamic studies as well as for therapeutic drug monitoring in clinical situations.
PLASMA PROTEINASE INHIBITOR POTENTIAL (PROTEIN C; PROTEIN C COFACTOR; PROTEIN S AND ANTITHROMBIN III) IN PATIENTS WITH NEPHROTIC SYNDROME

M. Siebels, J. Sis, K. Andrassy

The high incidence of thromboembolic complications in patients with nephrotic syndrome (NS) and the known relationship between thromboembolism and protein $\mathrm{C} / \mathrm{S}$ or antithrombin III (AT III) deficiencies prompted us to study this relationship in more detail. We were particularly interested to examine whether a deficiency of protein C cofactor was demonstrable. 15 patients with NS $(9$ males, 6 females; mean age 47 years; mean proteinuria $6.6 \mathrm{~g} / \mathrm{day}$; mean creatinine 1.49 $\mathrm{mg} / \mathrm{dl}$ ) who had no medication affecting haemostasis were analyzed. Patients with renal diseases but without NS served as controls. Protein $\mathrm{C}$ was analyzed with a chromogenic substrate (Immuno), protein $\mathrm{C}$ cofactor with functional assay (Haemochrom), protein S with ELISA (Immuno) and AT III with a chromogenic substrate (Kabi). As a result protein $\mathrm{C}$ activity (mean $118.6 \pm 10.8 \%$ ) as well as protein $\mathrm{S}$ concentration (mean $145 \pm 25 \%$ ) were within normal range in all patients. Protein $\mathrm{C}$ cofactor ratio was normal in $11 / 15$ patients but decreased in $4 / 15$ patients (mean 1.6). 1/4 patient with decreased protein $\mathrm{C}$ cofactor ratio had relapsing thromboembolic complications. AT III levels remained in the normal range (mean $108.8 \pm 10.3 \%$ ) in all patients.

CONCLUSION: Low protein C cofactor in NS has not been described before and may predispose to thomboembolic complications. In contrast to the common opinion, AT III was not diminished in any patient. Protein $C$ and $S$ levels were within in the normal range, thus affording some protection against thromboembolism in NS.

Present address: Department of Medicine, University of Heidelberg, Bergheimerstr. 56a, 69115 Heidelberg

\section{6}

\section{Praevalence of Heparin Cofactor II Deficiency IN IDIOPATHIC THROMBOEMBOLIC DISEASE \\ E. Aygören-Pürsün, S. Ehrenforth, I. Scharrer}

Heparin Cofactor II (HC II) is a physiological, heparin-dependent thrombin inhibitor with homology to Antithrombin III (ATIII). As yet it could not be shown that, analogous to ATIII, a defiency of HCII is associated with an increased risk of thrombosis. The significance of HCII deficiency, possibly with a genetic predisposition, was first suggested in 1985 with the description of familial heterozygous HCII reduction associated with thrombosis. However, no extended studies have yet been reported on the praevalence of reduced HCII levels in patients with thromboembolism.

In order to investigate the role of $\mathrm{HCII}$ deficiency as a risk marker for thrombosis, we determined the praevalence of HCII deficiency in a large group of patients with thromboembolic disease. ATIIl levels as well as activators and inhibitors of coagulation and fibrinolysis were measured simultanously in order to evaluate additional risk factors. 194 healthy volunteers served as controls. HCII antigen levels were assessed by immunoelectrophoresis according to Laurell.

The distribution of $\mathrm{HCII}$ plasma levels in 1075 patients ranged from 25 $260 \% .22$ males $(4.4 \%)$ and 70 females $(12.2 \%)$ showed HCII levels below the $95 \%$-reference interval, whereas still $8 \mathrm{male}(1.6 \%)$ and 27 female patients ( $4.7 \%$ ) exhibited values beneath the lowest level measured in the control group. This results in an overall praevalence of HCII deficiency of $8.6 \%$, resp. $3.3 \%$ according to the more strict definition, in a large group of patients with thromboembolic disease.

In conclusion, the results of the study could indicate that the significance of HCII deficiency has been underestimated in the past and that HCII will possibly gain importance as a thrombophilic risk parameter.

Dept. of Angiology, University Hospital Frankfurt, Theodor-Stern-Kai 7, 60596 Frankfurt, Germany 
"REVERSED FIBRIN PLATE ASSAY", A NEW GLOBAL TEST OF FIBRINOLYSIS

U. Wentscher ${ }^{*}$, I. Rohner, B. Krumpholz, R. Egbring, R. Seitz

Disturbances of the natural fibrinolytic potential are thought to be a major cause of thromboembolic diseases, but the available tests including measurement of plasminogen activators (PA) and their inhibitors (PAI), yielded conflicting results. In this study, a new global test of fibrinolysis, a modification of a previously reported (Seitz et al., Thromb Res 42:277;1986) "reversed" fibrin plate test was evaluated.

In contrast to the classical test used to measure the PA content of a plasma sample, in the "reversed" fibrin plate assay, a fibrin plate is prepared from the patient's plasma (by addition of agar, thrombin and calcium ions). Into a well punched into this fibrin plate, $1000 \mathrm{U}$ urokinase is pipetted, and the lysed area is measured after 24 hours incubation at $37^{\circ} \mathrm{C}$. The purpose of this "reversed" assay is to assess the susceptibility of the patient's fibrin to lysis, permitting the influence of all natural determinants of lysis (e.g. fibrin structure and cross linking by factor XIII, plasminogen activation, PA and plasmin inhibitors).

The test showed good precision (intra series VC $4.4 \%$ ) and reproducibility (inter series VC $4.7 \%$ ). The values were stable in samples frozen at $-25^{\circ} \mathrm{C}$ up to 4 months. In vitro, supra physiologic addition of $F$ XIII (up to $3 \mathrm{U} / \mathrm{ml}$ ) decreased lysed areas, verifying the influence of fibrin stabilisation on clot resistance. Cumarin plasma samples were within the range of a control group, heparin plasma can be used only after prior heparin neutralisation. A further clinical evaluation of the test appears to be worthwhile.

Philipps-University Hospitals, Centre of Internal Medicine ("at present: Centre of Dermatology) , Baldinger Str., D-35033 Marburg, Germany.

\section{9}

PLATELET COUNTS : A STANDARDIZED STATISTICAL PROCEDURE FOR THE DEFINITION OF THE LIMITS OF RELIABLE MEASUREMENTS

Keller, B. ${ }^{1}$, Hänseler, E. ${ }^{2}$, Rüegg, R. ${ }^{3}$, Keller, H. ${ }^{2}$

For many diagnostic and/or therapeutic decisions a reliable platelet count is of utmost importance. In cases of low particle concentrations, e.g. $<30^{\prime} 000 / \mu \mathrm{L}$, where the reliability of counting is crucial, increasing difficulties appear with visual as well as with electronic counting. It is astonishing that for most particle counters the lower limits of reliable counting are not given by the manufacturers. Maybe, because there is no concensus about the (statistical) definition of the measuring (analytical) limits. We propose here a statistical model for the assessment of these limits which is based on the variance function. It can be used for different applications in laboratory medicine. The practical use is demonstrated for platelets counted in triplicates with the Technicon Hl. Unselected EDTA blood samples $(N=150)$ where used for this investigation.

In the first step a so called 'imprecision profile', derived from the variance function was constructed in a range of $2000-500^{\prime} 000 \mathrm{plt} / \mu \mathrm{L}$. From this precision profile the lower limit of quantification (LLO) can be obtained. This is the limit, above which the result of a platelet count can be measured with a defined, from a clinical point of view acceptable coefficient of variation. In addition the variance function was used to extrapolate: 1) the 'critical limit' (LC), which is the point where the probability for a result $>0$ is $1: 1 ; 2$ ) the limit of detection (LD), which represents the threshold at which a platelet count is $>0$ with a probability of $95 \% ; 3$ ) the 'power of definition' (PD). The PD defines, for every concentration interval, a number of values which can be significantly (c.g. $p \geq 95 \%$ ) discriminated from each other. The values found for the $\mathrm{Hl}$ were $(\mathrm{plt} / \mu \mathrm{L}): \mathrm{LC}=4000 ; \mathrm{LD}=8200 ; \mathrm{LLQ}=$ $25^{\prime} 000$ (for a CV of $10 \%$ ). PD in the range of $20^{\prime} 000$ to $200^{\prime} 000$ was 14 .

The mathematical procedure proposed here does not rely on artificial standards or calibrators, can be applied for every instrument or analytical method, respectively and is easy to handle with a PC and a Microsoft Excel version 4.0. The programs are available on request.

Present addresses: 'Université Paris, UFR de Mathématiques, Paris, France, 2 Institute for Clinical Chemistry and ${ }^{3}$ Department for Intemal Medicine, Hematology, University Hospital Zürich, Switzerland

\section{8}

\section{FAMILIAL THROMBOPHILIA AND MYOCARDIAL INFARCTIONS ASSOCIATED WITH FAMILIAL} ELEVATION OF HISTIDINE-RICH GLYCOPROTEIN

\author{
E. Aygören-Pürsün, S. Ehrenforth, I. Scharrer
}

\begin{abstract}
Histidine-rich glycoprotein (HRG), a non-enzymatic alpha 2-glycoprotein present in human plasma and platelets has been reported to be involved in the fibrinolytic system and to interact with a number of proteins, including plasminogen, thrombospondin and fibrinogen, heparin, heparin cofactor II and protein C inhibitor-activity. Whether the elevation of HRG is a hereditary disorder which contributes to the pathogenesis of familial thrombophilia is at present a matter of disucssion, but until now there have been only few reports on inherited abnormalities of HRG. Therefore we would like to introduce a family, in which the combined occurrence of myocardial infarction resp. thrombophilia and high HRG plasma levels is striking.The characteristics of the family members, distributed over four generations, are shortly summarized as follows. The propositus has been healthy until the spontaneous occurrence of deep vein thrombosis, pulmonary embolism and myocardial infarction at the age of $53 \mathrm{ys}$. Her father and all five brothers suffered from myocardial infarction but only two of the brothers could be investigated and both had an elevation of HRG. Three brothers (III-V) and the father had died from cardiac arrest at an early age. The grandson had had cerebral vein thrombosis at the age of 2 years. The only abnormality observed in the propositus and other symptomatic family members was a persistently high level of HRG ( $>148 \%$, measured by EID/ Laurell) with normal levels of all other known thrombophilia parameters, including normal PAI- 1 activity. No factors causing acquired elevation of HRG were found in any of the subjects.

Consequently a hereditary HRG elevation is presumed in this family .
\end{abstract}

Dept. of Angiology, University Hospital Frankfurt, Germany

\section{0}

CLINICAL EVALUATION OF A NEW REAGENT (MULTIFIBREN U) FOR DETERMINATION OF FIBRINOGEN

L. Volbracht ${ }^{1}$, Th. Eller ${ }^{1}$, R.S. Ross ${ }^{1}$, K. Fickenscher ${ }^{2}$, C. Wagner 2 , D. Paar ${ }^{1}$

A newly developed reagent (Multifibren U, Behringwerke AG, Marburg, Germanyl for fibrinogen measurement based on the method by Clauss contains a specific inhibitor of fibrin aggregation and enables the use of undiluted samples. In this study we compared Multifibren $U$ method to our standard coagulometric fibrinogen determination using Multifibren (Behringwerke AG).

Materials and Methods: Opto-mechanical measurements using both Multifibren and Multifibren $U$ were done on Fibrintimer $A$ IBFA, Behringwerke AGI. Coagulometric fibrinogen determinations (Coagulometer KC 40, Amelung, Lemgo, Germany) were performed using Multifibren only. Reproducibility data were established using control plasmas (Kontrollplasma $N$, Kontrollplasma $P$, Behringwerke AG) and patient sámples.

Results: Precision (range $57-888 \mathrm{mg} / \mathrm{dl}$ ): Intra assay $(\mathrm{n}=8,5$ days, CVI: $1.3-3.3 \%$ for Multifibren U compared to $1.8-7.1 \%$ for Multifibren (BFA) and $2.2-3.3 \%$ for Multifibren on KC 40 ; Inter assay (5 days, $C V): 1.8-6.9 \%$ for Multifibren $U$ compared to $4.8-11 \%$ for Multifibren (BFA) and $3.7-6.4 \%$ on KC 40 . Linearity of Multifibren $U$ method: $70-900 \mathrm{mg} / \mathrm{dl}$. Method comparison (healthy subjects and patients, $N=176$, range $167-921 \mathrm{mg} / \mathrm{dl}$ ): regression analysis (Passing/Bablok): $Y=-63.2+1.15 X \quad(r=0.95)$ with Multifibren, $\mathrm{KC} 40(X)$ and Multifibren U. BFA (Y). Heparin influence on Multifibren $\mathrm{U}$ : insensitive up to $2.0 \mathrm{IU} / \mathrm{l}$ added.

Conclusion: Precision and method comparison data show comparable results for the fibrinogen determinations with Multifibren $U$ (BFA) and Multifibren (KC 40 ). The possibility of handling undiluted samples using Multifibren $U$ increases the practicability of fibrinogen determinations.

1 Division of Clinical Chemistry and Laboratory Diagnostics, Department of Internal Medicine, University of Essen, Hufelandstr. 55, 45122 Essen, Germany

2 Behringwerke AG, Marburg, Germany 
THE IDENTIFICATION OF FIBRINOGEN AS AN INTERFERING FACTOR IN A CHROMOGENIC HEPARIN ASSAY AND ITS PREVENTION

H.-J. Kolde, A. Stecher-Schilling, Baxter Diagnostics, Unterschleißheim

Recently we described the development of a functional assay for heparin or other drugs which inhibit the generation of thrombin (Wagenvoord et al, Hemoastasis $1993 ; 23: 26-37$ ). The assay was further simplified and could be fully automated. During the comparison of patients on heparin treatment we observed cases in which the new method gave much higher values than assays based on anti-factor $\mathrm{Xa}$ activity of heparin. We tried to identify the nature of interference by performing our thrombin generation inhibition assay (TGIA) at higher dilution of the samples. This partly lead to a reduction of the apparent heparin activity in the sample. By pretreating the samples with batroxobin we showed that the overestimation of heparin with the TGIA could be eliminated and that values with the anti-Xa assays were not influenced by the snake venom. Thus the fibrinogen of the sample was identified as the source for the overestimation of heparin in the TGLA. Most likely the first traces of the produced thrombin bind to fibrin monomers and are not longer able to activate factor V and VIII. In the next step we tried to overcome the effect of fibrinogen using different approaches. By preincubation of the patient plasma with antibodies against fibrinogen we could eliminate the pseudo heparin activity. Similar results were achieved by using the peptide GLY-PRO-ARG-PRO or derivatives with similar structure which are known to bind fibrin monomers. In order to simplify the assay systern we investigated if the addition of the peptide to the reagent was possible as well. As the result of these experiments we now lyophilize the peptide into reagent $A$ of the test kit. Thus the test remains as simple as described previously but with improved specificity. Based on these findings it should be evaluated if similar antithrombin effects of fibrinogen influence also other heparin sensitive test systems which are based on the gencration of thrombin such as the aPTT.

Address: Baxter Diagnostics, Scientific Support Hemostasis Europe, Edisonstraße 3, D-85716 Unterschleißheim

PROTEIN $z$ IN LIVER DISEASES

B. Kemkes-Matthes, K.J. Matthes

INTRODUCTION: Protein $\mathrm{z}$ is a Vitamin K-dependent plasma protein, which promotes the association of thrombin with phospholipid surfaces. In patients with bleeding tendency of unknown origin, deficiency states of protein $z$ recently have been shown by our group. In the present study, alterations of protein $z$ were investigated in patients with chronic liver diseases and compared to other vitamin $\mathrm{K}$-dependent coagulation proteins.

METHODS: Protein Z: ELISA protein $z$, Diagnostika Stago, France. Faktor IX: EIISA Factor IX-Antigen Test, Boehringer Mannheim, Germany. Protein C: ELISA Protein C Antigen Test, Boehringer Mannheim, Germany.

PATIENTS: 1 . Normals (N), $n=16 ; 2$. Chronic aggressive hepatitis (CAH), $n=16 ; 3$. Liver cirrhosis (CI), $n=18$.

RESULTS: 1. Protein $z: N: 2.800 \pm 330 \mathrm{ug} / 1(100 \pm 12$ z

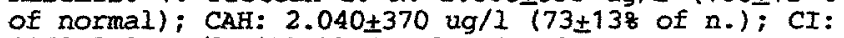
$1060 \pm 350 \mathrm{ug} / 1(38 \pm 12$ of $\mathrm{n.})$. 2. Factor IX: $\mathrm{N}$ : 100 \pm 14 ; CAH: $72+20$; CI: $54 \pm 12$ \& of normal; 3 . Pro-

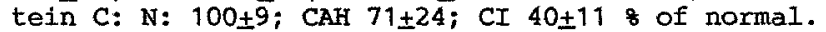
CONCLUSION: 1 . Protein $\mathrm{z}$ concentration decreases with increasing severity of liver disease. regression analysis shows highly significant correlations between protein $\mathrm{Z}$ and factor IX as well as between protein $\mathrm{z}$ and protein $\mathrm{C}$ indicating that protein $\mathrm{Z}$ is synthesized only by the liver. 2. Diminutions of protein $\mathrm{z}$ in severe liver diseases can contribute to the bleeding tendency of these patients.

Zentrum für Innere Medizin der Universität Gießen, Klinikstrasse 36 , D-35392 Giessen, Germany
ANTICARDIOLIPIN-ANTIBODIES IN ACUTE CEREBROVASCULAR DISORDERS AND SYSTEMIC LUPUS ERYTHEMATOSUS - EXPERIENCES WITH AN ENZYME-LINKED IMMUNOSORBENT ASSAY

Göppert $A^{1}, O t t M^{2}$, Wick $M^{1}$, Biniasch $O^{2}$, Haberl $R^{2}$, Samtleben $W^{3}$, Fateh-Moghadam Al.

Inst.f.clin.Chem. ${ }^{1}$, Dept. of Neurology ${ }^{2}$, I.Med.Dept. ${ }^{3}$ of the Klinikum Großhadern of the LMU Munich, FRG.

Anticardiolipin-antibodies (ACA) are considered to be risk factors for thromboembolic events. With a new, commercially available ELISA (elias Medizintechnik) we evaluated the significance of ACA as a risk factor for acute cerebrovascular disorders (ACD) and for thromboembolic events in SLE-patients. We determined ACA-IgG and -IgM in 95 patients with cerebral ischemia (CI; 81 stroke, 14 TIA) and 9 patients with cerebral venous thrombosis (CVT) and we compared the results with 59 patients with non-vascular or -autoimmune diseases of the CNS and 32 SLE-patients.

The interassay-coefficient of variation (CV) of the method was $17.4 \%$ for IgG and $15.3 \%$ for IgM, the intraassay-CV was $11.4 \%$ for IgG and $3.6 \%$ for IgM. The distribution of ACA in the control group was similar to an investigation of 1500 blood donors. $7(7 \%)$ of the Cl-patients were highly positive for ACA-IgG or -IgM (>5SD over the mean of the blood donors) and $2(3 \%)$ of the controls, statistically not significant. $3(33 \%)$ of the CVTpatients had low positive ACA-IgM (>2SD) and $2(3 \%)$ of the controls, statistically significant $(p<0.01) .13(40.6 \%)$ of the SLE-patients were highly positive for ACA-IgG or -IgM, a statistically significant difference compared to $\mathrm{CI}(\mathrm{p}<0.01)$ and to the control group $(p<0.05)$. These SLEpatients had more thromboembolic events than ACA-positive CI-patients. According to our results ACA are not a predominant risk factor for $\mathrm{ACD}$, however, ACA-positive SLE-patients seem to have an increased risk for thromboembolic events.
MULTICENTRE EVALUATION OF STA, AN AUTOMATED SELECTIVE ACCESS ANALYZER FOR COAGULATION TESTS

P. Hellstern (1), B.Faller (1), F.J.L.M. Haas (2), B.van Sterkenburg (2), W.Speiser (3), S.Handler (3), A. Hubbuch (4)

We report about the evaluation of STA according to theECCLS protocol. STA (distributor in Germany: Boehringer Mannheim $\mathrm{GmbH}$ ) is a multichannel selective access analyzer, designed for automated analyses of routine and special coagulation tests. Tests as follows were evaluated: PT, Hepato Quick, aPTT, fibrinogen (Clauss), thrombin time, AT IIl (chromogen), protein C (chromogen, clotting), protein S (clotting), as well as the factors II, V, VII, X, VIII $\mathrm{IX}, \mathrm{XI}$ and $\mathrm{XII}$.

Results: Within run CVs of the clotting tests were below $2 \%$ (calculated on the basis of seconds) in most cases, day to day CVs below $4 \%$, except for factors $V, I X, X I I$ and protein C. Chromogenic tests (AT III und Protein C) yielded within run CVs below $3 \%$ and day to day CVs below $5 \%$ in the decision range. Measuring ranges: AT III/Protein C chrom. : 20-140\%; fibrinogen: 1.3-9.0 g/l (plasma - dilution 1/20), after rerun with other dilutions: from $0.3 \mathrm{~g} / \mathrm{l}$ (dilution: $1 / 5$ ) to $18 \mathrm{~g} / \mathrm{l}$ (dilution: $1 / 40$ ) ; protein $\mathrm{C}$ und $\mathrm{S}$ (clotting) : $20-120 \%$.

Correfations with $\mathrm{KC10} \mathrm{A}$ were $>0.98$ when the same reagents were used on both instruments (tests: PT, Hepato Quick, aPTT, thrombin time). In comparisons with other reagents, results of the same quality were obtained with fibrinogen, AT III, protein C and in case of PT or Hepato Quick when reulis are in INR units. Sample related carry over was not observed up to $5.5 \mathrm{IU} / \mathrm{ml}$ heparin. Reagent related carry over was not observed with routine tests; the endogenic factors VIII, $\mathrm{X}, \mathrm{XI}, \mathrm{XI1}$, the protein $\mathrm{C}$ and $\mathrm{S}$ clotting reagents should be measured in a separate run. Throughput: 260 tests/h when PT, aPTT, fibrinogen und thrombin time are requested from each plasma: 190 samples are measured by STA within $3 \mathrm{~h}$, if the above mentioned profile is requested from each sample. No user intervention is necessary during that time interval. In conclusion, sta allows precise measurements even of rather long (pathological) clotting times, due to the optimized electromechanical clot detection principle. It is also a reliable system for photometric tests and well suited for lange workloads as well as STAT analyses of haemostasis tests.

Adresses: 1: Klinikum der Stadt Ludwigshafen (D); 2: St.Antonius Ziekenhuis, Nieuwegein (NL); 3:AKH Wien, Wien (A); 4: Boehringer Mannheim GmbH, Mannheim (D). 
INVITRO PLASMA DILUTION AND COAGULATION FACIOR DEFICIENCIES IN LUPUS ANTICOAGULANT

P. Brauer, W. Sibrowski, P. Zürrlein, F. Keller

Plasma samples containing lupus anticoagulant activity often show multiple coagulation factor deficiencies. In several reports these deficiencies are attributed to be spurious, because they disappeared using heterologous plasma substrates in the assay or they revealed sensitivity to plasma dilution and - last not least - patients did not show any bleeding tendency.

In five patients suffering from lupus anticoagulant and one normal control we determined activities of coagulation factors II, VII, X, VIII, $|X, X|$ and $X I I$. All determinations were performed with plasma dilutions of 1:1,1:2,1:4, 1:8 and 1:16 in DBA-buffer.

We found multiple simultaneous factor deficiencies almost exclusive in the endogenous coagulation system. All these activities showed a strong tendency to normalize in relation to plasma dilution. GeneralIy, the F. VIII increase with plasma dilution was most striking and in one case a rise from $<1 \%$ to $208 \%$ was observed.

Only plasma samples from one patient revealed a significant decrease of an exogenous coagulation factor ( $F . \|=19 \%$ ). This prothrombin deficiency did not show any increase with plasma dilution. interestingly. this patient was the only one suffering from bleeding tendency.

In conclusion, determinations of coagulation factor activities in different plasma dilutions are suitable to detect a lupus like coagulation factor deficiency' which is probably due to a diminished concentration of available phospholipids in the assay and does not indicate a real coagulation factor deficiency. Missing sensitivity to plasma dilution in this field might be a hint for a clinical relevant bleeding disorder

Institute for transfusion medicine

Albert-Schweitzer-Straße 33

D-48129 Münster
297 IN VITRO INFLUENCE OF IONIC AND NONIONIC CONTRAST TION

E. Lindhoff-Last, E. Tholouli, R. Schräder, G. Mosch,

K. Breddin

Ionic contrast media possess an anticoagulant effect in vitro and ex vivo. Nonionic contrast media seem to inhibit coagulation much less and sometimes have been made responsible for thromboembolic events during coronary angiography.

We have investigated the in vitro effects of ionic and nonionic contrast media on thrombelastography and on thrombocyte func-

tion (ADP- calcium plays an important role in coagulation and may be bound to ionic contrast media we also investigated their influence on coagulation after addition of ionic or nonionic contrast media.

Blood samples were taken from 10 young healthy donors.

The different contrast media (amidotrizoat, ioxaglat, iopamidol) were diluted 1:20 with native freshly drawn whole blood.

Thrombelastography: The reaction time $r$ and the clot formation time $k$ were significantly prolonged with the ionic contrast media (amidotrizoat and ioxaglat) while the nonionic contrast medium (iopamidol) did not change the reaction time $r$ but shortened the clot formation time

Calcium in a concentration of $20 \mathrm{mval} / /$ shortened the reaction time in the blood samples containing iopamidol, while ioxaglat and amidgtrizoat in combination with calcium showed proligngad raaccium.

When the ADP induced platelet aggregation was studied, the maximal a the ADP induce plate mal amplitude (ma) was significanty reduced by all cor When the collagen induced agaregation was studied in PRP of 8 When the collagen induced aggregation was studied in PRP of 8 added.

in two other donors the maximal amplitude was decreased. lopamidol only shortly decreased the maximal amplitude to a small extent while ioxaglat decreased it significantly. Calcium didn't show an additional effect.

We conclude that high local concentrations of ionic contrast media inhibit platelet aggregation and clotting while nonionic contras media do not show these in vitro effects. However nonionic contrast media have no "activating effects" in the tests studied.

Division of Angiology Department of Internal Medicine, J. W. Goethe-University Frankfurt am Main

Deutscher Titel: In vitro Beeinflussung der Thrombelastographie und Thrombozytenfunktion durch ionische und nichtionische Röntgen
kontrastmittel
296

TETRAHYDROXYQUINONE AS A NEW ACTIVATOR FOR INTRINSIC BLOOD COAGULATION PATHWAY

Ted C.K. Lee, Leslie Motley, and Hermann Pelzer, Department of Hemostasis Research and Development, Baxter Diagnostics Inc., Miami, Florida 33152, USA

The activation of the Intrinsic Blood Coagulation Pathway is initiated by the interaction of an activator with Blood Coagulation Factor XII There are several known activators for the Intrinsic Blood Coagulation including Ellagic acid, Silica, and Sulfatides.

We have found 2,3,5,6-tetrahydroxy 1,4-quinone as a new activator for the Intrinsic Pathway of Blood Coagulation. This compound with a concentration range of 1 to $3 \mathrm{mM}$, in the presence of metal ions and phospholipid, activated the Intrinsic Pathway of Blood Coagulation. Metal ions are required for the activity of tetrahydroxyquinone, and cupric ion was the most effective for that purpose. The Activated Partial Thromboplastin Time of the tested plasma samples mixed with the tetrahydroxyquinone based reagent was about 29 seconds. Plasma samples deficient of Factors XII, IX, or VIII did not respond to the activating agent in the clotting assay, indicating the usefulness of the reagent for the detection of the disorders of Intrinsic Blood Coagulation.

We tested a structural analog of tetrahydroxyquinone, a disodium salt of Rhodizonic acid. The Rhodizonic acid based reagent showed a low level activity for Intrinsic Blood Coagulation.

\section{8}

COMPUTER AIDED REAL TIME ANALYSIS OF THROMBELASTOGRAPHY

A. Calatzis, A. Stemberger, G. Blümel

The thrombelastography (TEG) was developed in the fifties by Hartert as an overall clotting assay to analyze hemostasis. A diagram similar to a tuning fork incorporates coagulation cascade, platelet function and fibrinolysis. Althoug modern coagulation procedures have superseded this method, a renaissance can presently seen in liver transplantation in the USA. The aim of this study group was to modify existing instruments (Thrombelstography $D$, Hellige, Freiburg) by a computer aided real time analysis. The signals are read into the computer using an analog digital converter. The computer programm displays 4 channels simultaneously on the screen of a $20 "$ monitor, which is about the same size as the original recorder system. The known parameters reaction time ( $r)$, coagulation $(r+k)$, maximal amplitude (max) and maximal elasticity (mE) are calculated compared to standard values and saved by the computer programm. At the present time 4 measurements begun at staggered times can be seen on the display during surgery, additional channels are in preparation. Supported by this data surgeons and anesthesists can initiate therapy during surgery on bleeding parenchymatous organs. 
CLINICAL HAEMOSTASIS AND VON WILLEBRAND FACTOR IN PATIENTS WITH CHRONIC RENAL. FAILURE UNDERGOING HAEMODIALYSIS

C.Reifenrath, R.Voss, F.Lübbecke, V.Wizemann

Coagulation disorders are a common problem in haemodialysis patients. Within a study of non-diabetic risk factors of coronary artery disease in 28 patients undergoing haemodialysis an investigation has been made concerning clinical and laboratory parameters of haemostasis.

Clinical data were obtained by a detailed standardized questionnaire including medical history. Patients were assigned to one of three groups according to their clinically obvious haemostatic state: patients with shunt- or other documented vessel thrombosis (THR), patients with bleeding tendency (BLE) (spontaneous haematoma and/or strong epistaxis) and patients with no clinically pathological haemostasis (NOR). F VII C and FVIII RAg were determined by ELISA (Behring) and RisCoF was assayed by formalin-fixed platelets (optical method). The actual status of clinical haemostasis under dialysis was: BLE 10/28, NOR $13 / 28$, THR $5 / 28$. Laboratory results were: platelets [G/1] BLE $208,8+1-22,85$ (s.e.m.), NOR $243,08+/-22,52$ (s.e.m.), THR $278,8+/$ 43,2 (s.e.m.); red blood cells [T//] BLE 3,42 +/-0,2 (s.e.m.), NOR 3,365 $+/-0,131$ (s.e.m.), THR 3,764+/-0,488 (s.e.m.); RisCoF BLE 178,2 $\div /-$ 17,66 (s.e.m.), NOR 225,0 +/-30,02 (s.e.m.), THR 230,0 +/- 11,04 (s.e.m.); FVIII RAg BLE 166,3 +/- 17,32 (s.e.m.), NOR 164,85 +/18,88 (s.e.m.), THR 199,4 +1-27,88 (s.e.m.), FVIII C BLE $92,6+1-7,6$ (s.e.m.), NOR 100,0+/-7,69 (s.e.m.), THR $119,8+1-18,07$ (s.e.m.) In the subgroup of patients with rhEPO-therapy $(n=22)$ clinical haemostasis was the following: before dialysis BLE $3 / 22$, NOR $18 / 22$, THR $1 / 22$; under dialysis: without rhEPO BLE $8 / 22$, NOR $13 / 22$, THR1/22; under ThEPO-therapy BLE 5/22, BLE (better under rhEPO-therapy) $4 / 22$, NOR 10/22, THR 3/22. Analysis of von Willebrand multimer structure is currently under investigation.

\section{Vascular Disease}

\section{0}

INFLUENCE OF STAPHYLOKINASE ON CLOT LYSIS, CLOTTING PARAMETERS AND PLATELETS IN VITRO

J. Hauptmann, I. Stebani and E. Glusa

Staphylokinase (SAK) is a bacterial plasminogen activator similar to streptokinase (SK) in its mode of action. Recombinant wild-type SAK and several variants are now available and have been shown fibrinolytically and thrombolytically effective. The fibrinolytic action in vitro is highly fibrin-specific.

In the present studies the fibrinolytic activity of recombinant SAK was compared to SK and TPA (Actilyse $\circledast$ ) in human plasma clot lysis systems in vitro. The parameters clot lysis, fibrinogen concentration, thrombin time and APTT were measured in plasma surrounding the clots. Human blood platelet aggregation was measured in plateletrich plasma (PRP) and whole blood using ADP and collagen as aggregation inducing agents.

After an incubation period of $4 \mathrm{hrs}$ SAK produced $50 \%$ clot lysis at final concentrations of $13 \mathrm{nmol} / \mathrm{l}$ and $\mathrm{PPA}$ at $8 \mathrm{nmol} / \mathrm{h}$, whereas SK did not produce $50 \%$ clot lysis up to $1000 \mathrm{nmol} / \mathrm{l}$.

At these concentrations residual fibrinogen was $99 \%$ in case of SAK, $98 \%$ in case of tPA and $<10 \%$ in case of SK

Reduction in fibrinogen by $50 \%$ was found at $7.2 \mathrm{nmol} / \mathrm{of} \mathrm{SK}, 50$ $\mathrm{nmol} / / \mathrm{l}$ of tPA and $750 \mathrm{nmol} / \mathrm{l}$ of SAK. The thrombin time and APPT were inversely correlated with residual fibrinogen in plasma. At $1000 \mathrm{nmol} / \mathrm{I}$ SAK did not influence ADP- or collagen-induced platelet aggregation in PRP and whole blood, whereas SK and IPA led to $40-60 \%$ inhibition., resp., of ADP-induced aggregation in PRP. Inhibition of aggregation correlated with decrease in thromboxane formation.

In summary, SAK is a potent fibrinolytic agent not compromising clotting or platelet function.

Institute of Pharmacology and Toxicology, Medical School Erfurt, Nordhäuser Str. 74, D-99089 Erfurt
301

ANTIPHOSPHOLIPID SYNDROME COMPLICATING CHRONIC GRAFT-VERSUSHOST DISEASE AFTER ALLOGENEIC BONE MARROW TRANSPLANTATION D. Söhngen ${ }^{1}$, A. Heyll ${ }^{1}$, G. Meckenstock ${ }^{1}$, W. Withold ${ }^{2}$, C. Specker ${ }^{1}, C$. Aul ${ }^{1}$, H. Wolf ${ }^{1}$, and $W$. Schneider ${ }^{1}$

${ }^{1}$ Dept. of Internal Medicine, ${ }^{2}$ Dept. of Clinical Chemistry, Heinrich-HeineUniversity, 40225 Düsseldorf (FRG)

Antiphospholipid antibodies (APA) are closely associated with arterial or venous thrombosis, recurrent fetal loss and thrombocytopenia in sysiemic lupus erythematosus and related autoimmune disorders. Beside other autoantibodies (e.g. antinuclear antibodies) APA have also been described in patients after allogeneic bone marrow transplantation (aBMT) in combination with graft-versushost disease (GVHD) and hepatic veno-occlusive disease (VOD). Whether APA may contribute to these disorders is still obscure.

We describe a 54-year-old man who received marrow transplant from his HLA-identical sister because of myelodysplastic syndrome (subtype according to FAB-classification: chronic myelomonocytic leukemia/CMML). Conditioning regime: cyclophosphamide $(120 \mathrm{mg} / \mathrm{kg}$ ), total body irradiation [10 Gy]; GVHD prophylaxis: cyclosporine (CsA; $4 \mathrm{mg} / \mathrm{kg} / \mathrm{day})$, short-term methotrexate. Although his clinical course was complicated by VOD, acute GVHD (Grade III), hematemesis and encephalitis after Herpes simplex infection marrow engraftment was confirmed on day +21 after aBMT. Seven months later CsA was withdrawn followed by severe chronic GVHD. Platelet count decreased to $10 \times 10^{9} / \mathrm{L}$, total protein increased to $10 \mathrm{~g} / \mathrm{L}$ showing an increased polyclonal gamma globulin fraction [lgG $4.83 \mathrm{~g} / \mathrm{L}$ ]. Additionally our patient developed antinuclear antibody $(1: 320$ in the indirect immunoflourescence test with a HEp-2cell line), anticardiolipin antibody $(27 \mathrm{U} / \mathrm{ml}$; ELISA for $\mathrm{lgG}$, postive rheumatoid factor $[93 \mathrm{lU} / \mathrm{ml}]$ and lupus anticoagulant (LA) LA was confirmed by prolonged aPTT [62 s], Kaolin clotting time $[160 \mathrm{~s}]$ and dilute Russel' s viper venom time [index 1.4], was not corrected by a 1:1 mixture with normal plasma, showed positive thromboplastin inhibition test [TTI/findex of 1.4] and positive platelet neutralization procedure (PNT); APA-ELISA showed LA of lgG-type. Bone marrow aspirate could exclude relapse and multiple myeloma respectively showing normal megakaryocyte count and mixed hematopoetic chimerism [17 metaphases showing $46, X X$ and 6 metaphases $46, X Y$ ]. Donor screening has been negative for all tests mentioned above. Therapy with high dose intravenous immunoglobulin and prednisone [2 $\mathrm{mg} / \mathrm{kg}$ for 10 days] was not effective. After beginning treatment with CsA $14 \mathrm{mg} / \mathrm{kg} / \mathrm{day}]$ platelet count and total protein became normal and all the autoantibodies vanished. Finally 18 months after aBMT treatment was stopped and the patient remained in complete remission.

From these data we conclude that $L A$ as other autoantibodies may contribute to chronic GVHD. Treatment with CSA seems to be more effective than high dose immunoglobulin or prednisone. 


\section{2}

Prothrombin Fragments $\mathbf{F} 1+2$ are Increased in Plasma after Coronary Stenting: Significance for Assessment of Subacute Thrombotic Stent Occlussion

M. Gawaz, F.J. Ncumann, I. Ott, A. Schömig

Cleavage of prothrombin into thrombin and formation of $\mathrm{FI}+2$ peptides represents the key reaction for fibrin generation. Recently, it has been postulated that evaluation of prothrombin fragments $\mathrm{F} 1+2$ in plasma might be a useful marker for imminent subacute thrombosis after coronary stenting. To test this hypothesis, a total of 50 patients were studied that were treated by implantation of Palmaz-Schatz stents for incomplete PTCA. All patients received daily $2 \times 100 \mathrm{mg}$ aspirin, phenprocoumon, and heparin to adjust PTT values of $80-120 \mathrm{sec}$ until the target INR range of 3.0 was achicved. F1+2 was measured before, just after, and then daily for a total of 14 days after implantation of stent. Subacute stent thrombosis occurred in one patient on day 4. Plasmatic concentrations of $\mathrm{F} 1+2$ (normal range: $<1.1 \mathrm{nmol} / \mathrm{L}$ ) were the highest just after coronary stenting (median (quartile): 0.71 $(0.56 ; 1.3) \mathrm{nmol} / \mathrm{L}$ ) and decreased thereafter, continuously (day 14 : $0.3(0.26 ; 0.41) \mathrm{nmol} / \mathrm{L}) .36 \%$ of patients showed one or several $\mathrm{F} 1+2$ values above normal range during the total time period of the study, in $28 \%$ of patients $\mathrm{F} 1+2$ values were above normal range after the first 3 days. In the patient with subacute stent thrombosis $\mathrm{F} 1+2$ plasma concentration just after coronary stenting was 1.47 nmol/L (84th percentile of the study group) and 16 bours before stent thrombosis $0.71 \mathrm{nmol} / \mathrm{L}$ (60th percentile).

We conclude that an increase of prothrombin peptides $\mathrm{F} 1+2$ in plasma after coronary stenting presents with a poor specificity for imminent coronary stent thrombosis.

I. Medizinische Klinik der Technischen Universität München, Germany

\section{3}

ANTITHROMBIN III AND GLYCAEMIC CONTROL IN NON-INSULIN DEPENDENT DIABETES MELLITUS (NIDDM)

K.M. Reinhardt, M. Steiner, H.-R. Nagel, C. Burstein, O. Anders\#, B. Ernst

Changes within the blood coagulation and fibrinolysis systems in diabetic patients appear to be affected by metabolic control. Since data on the relationship between glycaemic control and coagulation inhibitors in NIDDM are rather limited we have investigated 60 type II diabetics who had never used insulin divided into two groups: 25 patients in good glycaemic control (glycated haemoglobin below 7.3 $\%)$ and 35 patients in poor glycaemic control. Antithrombin III activity (ACL 3000, Instrumentation Laboratory) and antigen (BN 100 . Behringl were measured. The mean antithrombin III activity in all patients was $95 \%(S D=15 \%)$. However, poorly controlled type II diabetics were found to have significantly reduced antithrombin III activity $(91 \pm 12 \%)$ in comparison to patients in good metabolic control $(100 \pm 17 \%, p=0.016)$. The mean antigenic antithrombin III obtained from all patients was $0.304 \mathrm{~g} / \mathrm{l}(\mathrm{SD}=0.037 \mathrm{~g} / \mathrm{l}$ ) and is considered to be unreduced relative to controls. Patients in good metabolic control did not differ in their antigenic antithrombin III from patients in poor glycaemic control $10.309 \pm 0.040 \mathrm{~g} / \mathrm{h}$ vs 0.301 $\pm 0.034 \mathrm{~g} / 1)$. Antithrombin III activity demonstrated strong inverse correlation with glycated haemoglobin $(r=-0.38, p<0.005)$ whereas antigenic antithrombin III did not show any relation to glycated haemoglobin. These data suggest that reduced antithrombin III activity in poorly controlled NIDDM patients is directly related to glycaemic control. Loss of activity by non-enzymatic glycation appears to be involved rather than changes in antithrombin III concentration.

University of Rostock, Institute of Clinical Chemistry \& Pathobiochemistry, Department of Internal Medicine ${ }^{\#}$, Heydemann-Str. 6 , 18055 Rostock, Germany

\section{4}

LOCAL ACTIVATION OF FIBRINOLYSIS BY TOURNIQUET ISCHEMIA H. -G. Breyer, J. Fareed*, J. Lohbrunner, and A. Paech

Tourniquet ischemia is important for good survey and saving blood in orthopaedic surgery. There is a controversial discussion about the question whether tourniquet ischemia supports or reduces the risk of DVT. 55 patients with planned operations on the knee of at least $60 \mathrm{~min}$. where included in an open prospective randonized trial: Group A: with tourniquet using an Esmarch bandage, Group B: with tourniquet after elevation of the lower limb, and Group $C$ : without tourniquet. Samples of venous blood were collected simultaneously from a cubital vein and from the femoral vein of the operated leg: 1. prior to the operation, 2 . with beginning of anaesthesia, 3. while opening the tourniquet, and $30 \mathrm{~min}$ and 2 hrs later, and on the 1st, $3 \mathrm{rd}$ and 7 th postop. day. pH, pCO2, p02, t-PA-activity, PAI, FDP, FbD, and TDP where analyzed in all samples. The results show a local high $t$-PA-activity immediately after opening of tourniquet in group $A$ and $B$, which decreases within 30 min. FDP and FDDP levels increase also during operation when tourniquet is used, whereas PAI levels are normal. It is concluded that tourniquet ischemia increases local fibrinolysis in the operated limb, which probably prevents from DVT. DVT, controlled by colour-coded doppler ultrasound did not occur in any of the 55 pts.

* Dpt. of Pathology, Loyola Universitiy Medical Center, Maywood, USA

Dept. of Trauma Surgery, St. Gertrauden Hospital, Paretzer Str. 12, 10713 Berlin, FRG
305

RELATION OF PLASMINOGEN ACTIVATOR INHIBITOR ACTIVITY AND PLAMINOGEN ACTIVATOR INHIBITOR CONCENTRATION IN DIFFERENT PLASMA SAMPLES

G. Siegert, T. Fritz, E. Runge

Elevated plasminogen activator inhibitor (PAl) levels are associated with myocardial infarction and coronary artery disease. Three forms of PAl-1 are found in the circulating plasma: the free active PAI, the latent form of PAI and the inactive form of t-PA-PAl-complex. Therefor the PAI activity as well as the PAI concentration were determined in 150 plasma samples of patients of the Policlinical Department of Internal Medicine. The PAI activity was measured by chromogenic substrate assay (Behringwerke), the concentration of the free active PAI- 1 antigen was determined by the Actibind (B) PAI1 assay (Technoctone) and the concentration of the whole PAl-1 was measured by the PAI-1 Elisa (Technoclone). Blood was anticoagulated by the low $\mathrm{pH}$ citrate anticoagulant Stabilyte@ (Biopool) for preventing the formation of t-PA-PAI complexes and the release of PAI from platelets after blood sampling. The PAl activity was strongly correlated with the free PAl antigen concentration $(r=0,932 ; p<0,001)$. Both PAl activity values were similarly correlated to the PAl-1 concentration. The very high range of the ratio of free PAl-1 antigen / whole PAl-1 antigen $(0,18-0,92)$ indicates a very high difference between the three plasma forms of PAI.

Present address: Dr. med. habil. G. Siegert

Technical University Dresden

Faculty of Medicine Carl Gustav Carus

Institute of Clinical Chemistry and Laboratory Medicine

Fetscherstraße 74

01307 Dresden 
306

ASSOCIATION OF COAGULATION FACTOR VII AND LIPOPROTEIN METABOLISM PARAMETERS

G. Siegert, M. Kotzsch, S. Albrecht, T. Luther, S. Bergmann, U. Julius, A. Petzold, I. Hoche

Prospective epidemiological studies indicate that elevated levels of factor VII are implicate as risk factor in cardiovascular disorders. The association of factor VII activity measured by one-stage clotting assay (VIic) and factor VIi concentration (ELISA) and their relationship to lipoprotein metabolism parameters as well as tissue factor apoprotein concentration (apo-TF) was studied in 400 plasma samples of normolipaemic probands and in 970 hyperlipaemic patients. In 700 plasma samples the factor VII activity was also measured by chromgenic substrate assay (VII chrom). Factor VII activity as well as factor VII concentration were higher in hyperlipaemics than in normolipaemics. The highest factor VII values were obtained in patients with combined hyperlipoproteinemia (EAS type D). Bivariate statistical analysis showed a significant correlation between factor VII activity and factor VII concentration $(p<0,001)$. For both parameters a significant positive relationship to the concentration of total cholesterol (TC). iriglycerides (TG), LDL-cholesterod as well as apoplipoprotein B was found. The factor VIlc activity was significantly higher than factor VII chrom activity in all groups. The highest difference between both factor VII activities was obtained in plasma samples of patients with combined hyperlipoproteinernia. No correlation was observed between apo-TF and factor VII activity as well as factor VII concentration. Likewise, there was no association between the concentration of prothrombin fragments $F 1+2$ and all other parameters measured in a sub population of 200 plasma samples. The association of factor VII activity and factor VII concentration to TC as well as TG suggested a close relationship between the concentration of lipoproteins and factor VIl synthesis as well as factor VII activation. There was no direct evidence that an increased activation of the coagulation cascade leads to higher Factor VII activity in patients with hyperlipoproteinemia.

Present address: Dr. med. habil. G. Siegert

Technical University Dresden

Faculty of Medicine Carl Gustav Carus

Institute of Clinical Chemistry and Laboratory Medicine

Fetscherstraße 74

01307 Dresden
308

EFFECT OF INTERMITTEND INTRAARTERIAL INFUSION THERAPY WITH A MIXTURE OF ADENOSINE TRIPHOSPHATE AND OTHER ENERGY-RICH PHOSPHATES (LAEVADOSINR) ON THE FIBRINOLYTIC POTENTIAL AND PLATELET FUNCTION IN PATIENTS WITH SEVERE CLAUDICATION

J. Pieper, M. Sosada, M. Barthels, A. Creutzig*, $R$. Alexander ${ }^{*}$ and $H$. Poliwoda.

In former studies we could not prove that the improvement of severe claudication by therapy with intraarterially admitted prostaglandin $\mathrm{E}$ was related to the fibrinolytic potential or platelet function. Similar successful treatment can be achieved by Laevadosin $R$. 10 patients $(\mathrm{m} / \mathrm{f}=8 / 2$, mean age $=58$, range $42-75$ years) received during a period of 14 days 24 infusions over 50 minutes with $10 \mathrm{ml}$ Laevadosin $R$ into the femoral artery. Before as well as 7 and 14 days after initiation of therapy พе determined t-PA, PAI-1, \& $2^{-}$ antiplasmin, plasminogen, D-dimer, fibrinogen (clauss) and thrombometer time. Significant elevated levels of plasminogen, D-dimer and fibrinogen were observed before therapy. During therapy fibrinogen levels decreased from 4,57t 0.8 to $3,7 \pm 1,0 \mathrm{~g} / 1 \quad(\mathrm{p}<0,05)$. Plasminogen levels increased in the same period from $138,67 \pm 29,01$ to $154,33 \pm 31,49 \approx(p<0,05)$. The other above mentionned parameters were in the respective normal range before and at the end of the two week lasting therapy. In Laevadosin ${ }^{R}$ treated patients the increase of plasminogen could indicate an activation of the fibrinolytic system.

Div. of Hematology and oncology, Div. of Angiology*, Hannover Medical School, D-30623 Hannover

V. HACH-WUNDERLR, S. BNDERRMANN, R. VANDERPUYE, H.W. NRSTLE, CH. SCHWAHN SCHREIBER, H. JORGALL. group 1 ( $n=9$ ) the distal anastomosis was located above and in aroup 2 in (11) belon the knee. Blood was first taken from the femoral artary before clamping (I pre-occlusiva value) and secondly from the popliteal vein in grous 1 or from a crural vein in group 2 imediately before declanping the bypass (E intra-acclusive values). Mean claping tiw was 57+1-21 uin in group 1 and $90+1-43$ min in group 2

As expected there was fall of pO2 $(108+/-32$ to $26+/-7$ mitg) with a parallal incraan of pcos $(43+/-5$ to $56+/-10$ mfg) and lactate $(8.4+/-3.1$

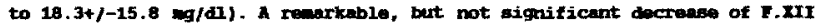
was fornd $(74+/-27$ t to $32+/-29 *)$. T-Ph-activity incransed from $0.03+/-0.02$ before to $0.12+1-0.06 \mathrm{ng} / \mathrm{m}$ during clawing. Alteraticrs of all parmesters ware growter in group 2 as compered to group 1.

Oniy alight dacreases were detected for F.VIII: C $(148+/-48$ to $78+/-59$ i) and F.V $(101+/-218$ to $84+/ 25 *)$ well as for InT-complexes $(24+/-24$ to $23+/-21 \mathrm{\mu g} / \mathrm{al})$ and prothrombin fragents $1+2(2.0+/-1.7$ to $1.9+/-0.7$ nool11), Several othex proteirus ghowed only minimul changes, $-g$. fibrinogen, platelets and leucocytes as well as protein C, protein S, von Willebrand-factor and PAI-1.

These results sugeast that activation of the contact systen and liberation of ondothelial-darived proteases of the fibrinolytic systen are dependent on the degree of ischeia and/or the location of the distal anastomosie of the arterial bypass. 
309

DEEP VEIN THROMBOSIS, DICOUMAROL NECROSIS OF THE LEFT MAMMA IN A WOMAN WITH PARTIALLY F XII DEFICIENCY

U. Köhler, W.B. Schwerk, K. Görg, R. Seitz, M. Klausmann, R. Egbring, P.v. Wichert

Coumarin skin necrosis is an uncommon complication which usually may occur between the 3-7 day after anset of treatment with vit $k$ antagonist's. In congenital disorders with thrombophilia as in protein $C+S$ deficiency, even in patients (pts) with dysfibrinogenaemia and in pts with heparin treatment or heparin associate thrombopenia (HAT ${ }_{2}$ ) skin necros is had been observed more of ten than in normat human beings.

He would now like to report about a very severe skin necrosis of the breast in a womian born in 1955 with partial F XIl deficiency ( $30 \%$ of normal).

four weeks after immobilisation due to calf muscle pulting a $\checkmark$. femoralis sinistre thrombosis were found by phlebography.

Ul tra high throabolysis with 9 Mitl. Sx for 3 days leeds to total recanal isation of the vessels as follow-u studies with ultra sonic examination demonstrated. Four days after starting an oral anticoagulant treatment (AKT) with dicoumarol a severe coumarin necrosis developed on the left manma.

Therapeutical doses of heparin (bolus $=5000 \mathrm{jU}$ ) liquerin and infusion of 30 40.000 IU heparin/daily and transitory ATIII supplementation improves the prenecrotic state.

U.S. examination show a large inflamatory infiltration of the fatty $t$ issue and the glandula mamaria sinistra.

While the ATIII substitution was two times discontinued the AIIII level was found to be lower during the intervals.

The prenecrotic skin recovered, but a strong induration of the deep infittrations renain. Three months later some of the infiltration became liquid.

Discussion: Disturbances in microcirculation with microthrombosis should be responsible for the inflamatory skin reaction and consecutive necrosis of the skin and in particular of the fatty tissue. A restitutio ad integrum can only be achived if the therapeutic procedere can started as quickty after the beginning of the symptons due to the inflamatory tissue reaktions.

\section{EFFECT OF FIBRINOGEN SUBSTITUTION ON BLOOD} VISCOSITY AND PLATELET FUNCTION IN AFIBRINOGENEMIA - A CASE REPORT

Armin J. Reininger ${ }^{1}$, Cornelia B. Reininger ${ }^{2}$. Michael Spannagl ${ }^{3}$, Ulrich Heinzmann ${ }^{4}$, Laurenz $\mathrm{J}$. Wurzinger ${ }^{1}$

The 31 year old afibrinogenemic female patient exhibited a history of severe bleeding since childhood. Upon in vitro addition of fibrinogen to the patient plasma normal extrinsic and intrinsic coagulation pathways were verified. Prior to and following fibrinogen substitution (1-2 g) - performed due to acute bleeding - platelet function as well as blood viscosity and density were examined.

Before substitution, plasma fibrinogen levels, prothrombin time, aPTT and thrombin time were not measurable. Within $30 \mathrm{~min}$ after substitution fibrinogen was detectable in the patient plasma $(0.15 \mathrm{mg} / \mathrm{ml})$ and remained so for up to three hours. In contrast, prothrombin time, aPTT and thrombin time reached normal values and were measurable for up to 30 hours. Plasma and whole blood viscosity and density remained unchanged following substitution. Platelet aggregation (light transmission aggregometry) induced with agonists requiring fibrinogen as a ligand was pathologic before and normal after substitution. Spontaneous platelet adhesion as measured with a novel method for platelet function testing the Stagnation Point Adhesio- Aggregometer (SPAA) - was absent prior to substitution. One hour following infusion, adhesion became detectable and values within the normal range were obtained. Spontaneous aggregation was not observed. Scanning electron microscopy revealed adhering platelets exhibiting normal pseudopodia. Furthermore platelet spreading, which is more surface specific than initial attachment, was identical to that observed with controls.

Our results indicate that fibrinogen substitution in an afibrinogenemic patient transiently corrected plasma fibrinogen concentration to a degree sufficient for normalization of coagulation as well as platelet function. Conversely, blood viscosity and density were not affected and thus appeared to be determined by plasma proteins other than fibrinogen.

1 Anatomisches Institut, Technische Universität München, Biedersteinerstr. 29, 80802 München, Germany, ${ }^{2}$ Chirurgische Klinik und Poliklinik und 3 Medizinische Klinik Innenstadt, Ludwig-Maximilians-Universität München, ${ }^{4}$ GSF-Forschungszentrum, Institut f. Pathologie, Neuherberg
311

TRANSIENT, ACQUIRED VON WILLEBRAND'S DISEASE F. Bergmann, L. Grote, M. Barthels, M. Sosada und H. Poliwoda

Acquired von Willebrand factor ( $V W F$ ) deficiencies have been described in myeloproliferative disorders, autoimmune disease as well as drug induced, e.g. valproic acid. In some instances a transient inhibitor could be detected.

A 29 year old woman was hospitalized for unexplained, multiple hematomas developing within one week. She never had any bleeding tendency before and family history was not informative. The initial laboratory data revealed a prolonged aPTT of $53 \mathrm{~s}$ with a decreased FVIII level of $11 \%$. A FVIII- or lupus-inhibitor were excluded. Platelet count and aggregation studies, liver and kidney function tests as well as immunological data were normal. Further analysis showed the decrease of WWF/FVIII complex, with vWF:Ag $19 \%$, Ristocetin cofactor $<5 \%$ and abnormal multimeric pattern of VWF like in type II von Willebrand's disease (VWD). The thrombometer time was markedly prolonged with $55 \mathrm{~s}$ (normal range 25-35 s). Medical history revealed onset of hematomas six weeks after i.m. administration of diclophenac for treatment of back pain and four weeks after oral vitamin B12 supplementation. The patient has been taken ibuprofen and paracetamol already since years.

The vWF/FVIII complex returned to normal values over a two months period. An inhibitor to vWF, resulting in reduced functional activity, was not detectable. The patient was exposed to diclophenac suppositorium again. This time the vWF/FVIII complex was not influenced. The preparatory agent for the developing of VWD could not be defined.

Acquired vWD can be transient. Therefore, a single investigation of WWF parameters may not be sufficient to distinguish this form from inherited WWD.

Medical School of Hannover, Department of Medicine, Hem/Onc; 30623 Hannover, Germany.

\section{2}

VON UILLEBRAND DISEASE TYPE NORMANDY IN PHENDTYPIC MILD HAEMDPHILIA A AND VON UILLEBRAND DISEASE TYPE I

U. Budde, R. Schneppenheim, E. Drewke, S. Krey, E. Lechler, J. Oldenburg, F. Bergmann

Recently a new dysfunctional variant of von willebrand disease (vtld) was described, in which the tinding capacity of van willebrand factor (Vuf) for factor VIII (F VIII) is greatiy reduced. In most patients the disease mimics mild haemophilia A in that these patients show a markedly reduced F VIII, while the vuf concentration is well within the normal range. Some patients, however, are diagnosed as mild vwd type I with vef properties between 35 and $50 \%$, while F VIII $C$ is disproportionally low.

After development af a specific assay for this variant we determined the F VIII binding capacty of the vulf in 140 patients with hemophilia $A$ and vwd. Seven patients from six different families were diagnased with vwd type Normandy. The former diagnusis has been haemophilia $A$ in four patients, and vuld in the remaining three. Remarkably aut of nine patients with suspected haemophilia $A$ whose molecular analysis for mutations in the $F$ VIII gene was unsuccessful, three suffered from vidd type Normandy.

In one family the patient was diagnosed with vid type I with an additional $F$ VIII binding defect. His father displayed only the $F$ VIII binding defect, his mather had type I vidd with narmal F VIII binding-eapacity. The molecular analysis in this family identified the mutation T28M in exon 18 as the underlyng defect responsible for the deficient F VIII binding in the patient and his father, and a frameshift mutation in the same exan, being responsible far the reduced viff properties in the patient and his mother.

Supported by the Deutsche Forschungsgemeinschaft (grant Schn $325 / 2-1$ )

Blutspendedienst AK Harburg

EiBendorfer Pferdeweg 52

D 21075 Hamburg 
A CASE OF A LUPUS ANTICOAGULANT DETECTED BY AN ABNORMAL PROTHROMBIN TIME AND PATHOLOGICAL EXTRINSIC FACTOR ASSAYS WHEN USING A THOMBOPLASTIN BASED ON RECOMINANT HUMAN TISSUE FACTOR AND SYNTHETIC PHOSPHOLIPIDS

Marbach J, Bammel E, Rombach B, Kolde H-J

Evang, u. Johanniter Krankenanstalten Duisburg-Nord and Baxter Diagnostics, Scientific Support Haemostasis Europe, Unterschleissheim

Lupus anticoagulants (LA) are circulating autoantibodies directed against negatively charged phospholipids. Very often these antibodies are identified in phospholipid dependent coagulation assays such as the activated partial thromboplastin time (aPTT), in some cases also in the prothrombin time (PT). We identified a case of a LA in 68 year old patient with a history of various hematological disorders who was hospitalized for a surgical removal of a sequestered lumbal nucleus pulposa prolaps. This patient had a strongly pathological PT $(25 \%, 27,4 \mathrm{sec})$ when tested with a PT reagent based on recombinant human tissue factor (r-hTF) and synthetic phospholipids

(Innovin ${ }^{\text {th }}$ ) but a normal aPTT and fibrinogen. Surprisingly the PT with various tissue thromboplastins of either rabbit brain $(n=3)$ or human placenta $(\mathrm{n}=1)$ were normal. A second PT reagent based on r-hTF and purified natural phospholipids showed a PT activity of $54 \%(19,8 \mathrm{sec})$. Assays of the extrinsic factors were performed with Innovin and showed pathological results for all of the 4 extrinsic factors at the regular 1:10 dilution which normalized after the sample was diluted further. The presence of LA was confirmed by a diluted RVV assay at two different phospholipid concentrations and the diluted thromboplastin inhibition assay with Innovin. These results show that the presence of a lupus anticoagulant can strongly influence PT results and also extrinsic factor assays and that this effect may depend also on the reagent that was chosen. It shows also the importance of subsequent specific testing in order to determine the reason for pathological screening tests and to provide a rational base for specific treatment.

Address: Evangelische Krankenanstalten Duisburg-Nord, Med. Klinik, Frau Dr. Marbach, Fahrner Str. 135, 47169 Duisburg

LOW MOLECULAR WEIGHT HEPARIN (LMWH) CAN CAUSE HEPARIN. ASSOCIATED THROMBOCYTOPENIA (HAT)

A. Greinacher $r^{t}, K$. Langenheim ${ }^{2}, H$. Marquort ${ }^{2}$

\section{We report the case of a 67 year old woman who developed the} immunologic type of HAT during low dose treatment with LMWH following elective hip surgery. The patient had not been treated with heparin before. 13 days after commencement of LMWH platelet count dropped to $43 x$ $10^{9} / \mathrm{l}$. This was coincidenced by an embolic occlusion of the right femoral artery. Following cessation of all heparins platelet counts normalized. Diagnosis of HAT was verified by HIPA test.

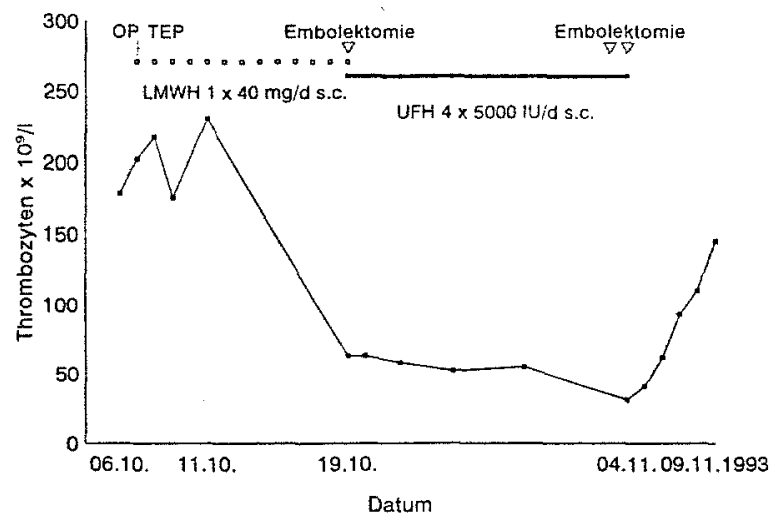

weibl., geb. 1926, Totalendoprothese rechte Hüfte, Wahteingriff

1 Institut für Klinische Immunologie und Transfusionsmedizin, JustusLiebig Universität, Langhansstr.7, 35385 Giessen.

2 Institut für Anaesthesiologie und operative Intensivmedizin, Ev.-Luth. Diakonissenanstalt Flensburg
SEVERE BLFEDING COMPLICATTONS DUE TO VITAMTN $K$ DEFICIENCY IN A PATIENT WITH OTHERWISE SYMPTOMLESS CELIAC SPRUE

K. Heidinger, B. Kemkes-Matthes, V, Franke*, K.J.Matthes, H. Heckers

CASE REPORT: A 47 year old patient was admitted to our hospital in june 1993 because of severe suffusions and haematomas in both arms and legs, particularly in both feet. No significant diseases had been noted before this event. At the time of admission activated partial thromboplastin time (aPTT) and prothrombine time (PT) were indefinitely prolonged. Vitamin $\mathrm{k}$-dependent coagulation proteins were below 1 \& of normal, while factor VIII:c was $60 \%$ of normal. The cause of the patients vitamin K-deficiency was initially not clear. The suspected ingestion of oral anticoagulants/ vitamin $K$ antagonists could not be confirmed. After substitution of vitamin $K$ and PPSB $i . v .$, PT- and aPTT-values normalized, however, during the following weeks PT decreased again. Vitamin A test showed no resorption of vitamin $A$, and endoscopic examination of small bowel revealed the macroscopic picture of celiac disease. A biopsy showed significant villous atrophy and lymphocytic infiltration of mucosa, consistent with the diagnosis. Presently the patient is on gluten free diet, receives vitamin $\mathrm{K}$ and other vitamin supplements (ADEK-FAL $R^{R}$ ). No further bleeding was observed, his clinical condition is stable, PT values are around $70 \%$ of normal.

CONCLUSION: Otherwise symptomless malabsorption due to celiac disease can lead to extreme vitamin $\mathrm{K}$ deficiency causing severe bleeding complications because of diminutions of all vitamin $\mathrm{k}$-dependent coagulation proteins below levels of detection.

zentrum für Innere Medizin und Zentrum für Pathologie* der Universität Gießen, Klinikstrasse 36. D-35392 Giessen, Germany.

COUMARIN NECROSIS IN PROTEIN S DEFICIENCY TYPE I B.Remkes-Matthes, M.Heidt, J.Seiboldt, K.J.Mat thes

INTRODUCTION: Protein $C$ deficiency is well known to be a risk factor for coumarin necrosis, but little is known concerning protein $s$ deficiency. We here report a case of protein $S$ deficiency type $I$ and severe coumarin necrosis. CASE REPORT: 43 year old male experienced spontaneous deep venous thrombosis of the left thigh in May 1992. Subsequently, he received oral anticoagulation until April 1993. In May 1993, deep venous thrombosis of the right leg occurred and coumarin treatment was restarted with the high dosage of $60 \mathrm{mg}$ phenprocoumon on the first two days. On the fourth day, coumarin necrosis occurred and the patient was referred to our center, presenting a large necrotic area from the right thigh to the calf. Coagulation studies showed normal AT III- and protein C-values (antigen and activity), but diminished protein $\mathrm{S}$ levels. In July 1993, the following values were measured: Protein S total $120 \%$ of normal, protein $S$ free 248 of total protein 5 , protein $S$ activity 40 \& of normal. The patients father and one of his children were also found to be protein $s$ deficient. After healing of the wound by skin transplant, coumarin treatment was restarted in August 1993 with $10 \mathrm{mg}$ warfarin (Coumadin ${ }^{R}$ ) daily. No further complications occurred, and prothrombin time was in the therapeutic range on the $10^{\text {th }}$ day. ConcuUSIon: Protein $s$ deficiency type I as well as protein $C$ deficiency are risk factors for coumarin necrosis. In our patient, the extremly high initial dosage of phenprocoumon may have been an additional risk. We conclude that oral anticoagulant treatment generally should be started carefully with a low dosage of oral anticoagulants.

Zentrum für Innere Medizin der Universität Gießen, Klinikstrasse 36, D-35392 Giessen, Germany. 
317

\section{PHENPROCOUMON ASSOCIATED NECROTIC HEPATITIS - A CASE REPORT - \\ S. Ehrenforth, M.v. Depka, I. Scharrer}

In the literature, only few well-documented cases of phenprocoumon-induced liver toxicity have been reported. Liver bistology in these reports is mentioned only briefly. This report adds an interesting case with strong indications pointing to necrotic hepatits caused by Marcumar( exposure and the obvious improvement of the liver function by the following Sintrom administration.

A woman who had had a heart- valve replacement in 10/1991 at the age of 55 years, was put on long-term treatment with Marcumar(8. 7 months later the patient suffered from jaundice and exhaustion. A distinct increase of the liver enzymes and bilinubine and an decrease of cholinesterase was determined firstly. After discontinuation of Marcumar@ treatment and subsequently administration of heparin, the liver enzymes returned almost to normal. In view of a possible causal relationship between the administration of phenprocoumon and the development of hepatits, the pt. was reexposed to Marcumar ${ }^{\circledR}$. This reexpositon was followed by a renewed increase of the liver enzymes. Thus Marcumaris was again withdrawn and subsequently the enzymes became nearly normal. Two liver biopsies revealed acute inflammation with massive, mainly centrilobular parenchymal damage and a histologic picture as seen in acute drug-induced, necrotic hepatitis. Immunhistochemical and serological testing showed no signs of any viral infections or autoimmune disease, the abdominal ultrasonography was normal. In 10/92 the pt. was put on Fraxiparin( three times daily and her liver functions improved subsequently. Because of adverse reactions and intolerance to s.c. heparin, a last, short-time Marcuma® reexposure was tried in $6 / 93$, but followed by elevation of the liver enzymes again. Since 7/93 the pt. was treated with Sintrom (acenocoumarol), which led to a rapid drop of the liver enzymes. Under this treatment the pt. is doing well and no renewed liver dysfunction or other complications were observed as yet.

The reported case, which we would like to discuss in detail, strongly suggest a causal relationship between phenprocoumon and liver necrosis and thus indicates a regular testing of the liver enzymes in all patients under phenprocoumon treatment.

Dept. of Internal Medicine, University Hospital Frankfurt, Germany

\section{8}

SPLANCHNIC VEIN THROMBOSIS CAUSEO BY PILLS IN TWO YOUNG FEMALES FOLLOWED BY THE MANIFESTATION OF CHRONIC MYELOPROL IFERATIVE DISEASE (CMPD).

H. Losonczy, M. Dávid, I. Batthyányi, L. Horváth

A 20 and a 25 y.o. female patients were examined with clinical and iconographical signs of splanchnic vein thrombosis leading to portal hypertension. Previously both patients were on oral anticoncipients for 1 year. Haemostasis tests: PT, APTT, TT, fibrinogen, plasminogen AP, ECLT with venous acclusion, $t-P A$ and $P A I-1$ activities and the inhibitors (AT III, protein C, protein S activities and antigen levels) were determined. In the first patient a transient decrease of AT III activity, elevated fibrinogen level and decreased fibrinolytic activity could be detected. In the second patient decreased fibrinolysis was present. We decided to start anticoagulant therapy with heparin, later coumarin combined with Pentosan Polysulfate. In the first patient the thrombus in the inferior vena cava dissolved after 6 weeks of this therapy and the ascites and oedema of the legs had gone. Ascites disappeared also at the second patient. Two and one year later, respectively, CMPD manifested at both patients as a basic disease. IFN alfa $2,5 \mathrm{MU} / \mathrm{m}^{2}$, was introduced at both cases while maintaining the anticoagulant therapy. Clinical and iconographical improvement of the portal hypertension and CMPD proved the efficacy of our therapy. Every case of abdominal vein thrombosis must be checked for occult or overt form of CMPD.

First Department of Internal Medicine, Medical University of Pécs, $H-7624$, If júság $u$. 13.
319

COINCIDENCE OF HOMOCYSTINURIA AND ATRLAL. MYXOMA. A CASE REPORT AND REVIEW OF LITERATURE.

\section{G. Huhle, F.V. Leistikow, A. Scherhag, J Harenberg, D.L. Heene}

Since the first report on Homocystinuria, described in 1962 by Carson et al., clinical and analytical investigation of patients suffering on juvenile vascular disease have focussed on the role of homocysteine on arteriosclerotic and atherothrombotic disease.

Life threatening effects by hereditable and nonhereditable homocystinuria are caused by severe thrombotic and embolic episodes. Imoderate increase of homocysteine-concentration in blood induces endothelium lesion, prothrombin activation, reduces antithrombin III levels and coagulation-factor VII synthesis.

Rare forms of haemocystinuria are found in patients with vitamin $B_{6}$ and vitamin $B_{12}$ - deficiency, chronical renal failure, in menopausal age, at oral contraception and in smokers.

Typical biochemical and clinical phenotype was seen in a young patient born in 1965 . Ectopia lentis and marfanoid features led to the diagnosis of inherited homocystinuria in 1971. Small activity of cystathionine-Bsynthetase was found and therapy was pyridoxal-6-phosphate. Nevertheless, episodes of venous and arterial thrombosis including lung embolisation were seen in the following years. In 1984 an atrial myxoma was diagnosted and operatively treated by excision.

However, coincidence of atrial myxoma and homocystinuria has not been reported so far. Following the interpretation of Thorel (1944), atrial myxoma-genesis is induced by thrombus formation. Ribbert (1924) reported on the concept of neoplasmatic transformation of endothelialcells responsible for myxoma formation.

The present case report implicates that the coincidence of homocystiuria and atrial myxoma is caused by toxic endothel-cell injury and concomitant coagulation abnormality.

I. Medizinische Klinik, Fakultät für Klinische Medizin Mannheim der Universität Heidelberg, Theodor-Kutzer-Ufer, D-68167 Mannheim

320

PROTHROMBIN MARBURG - A DYSFUNCTIONAL PROTHROMBIN DEFICIENCY?

K.H. Beck, A. Leonhard*, M. Kraus, , V. Kretschmer, and H.W. Seyberth

We report about prothrombin deficiency with less than $2.8 \%$ factor activity. Intraabdominal postpartal bleeding was observed in a newborn with $3.3 \mathrm{~kg}$ body weight. Bleeding was sonographically localized intraabdominal.

Analysis of clotting àctivity after finishing plasma therapy revealed a factor II (F II) activity below detection limit $(<2.8 \%)$. The activities of the other factors of the extrinsic and intrinsic systems as well as of protein C, protein $S$ and plasminogen were within the normal range for newborns. A factor II inhibitor could be excluded by plasma exchange tests. Investigation of the parents of the prop. showed reduced $\mathrm{F} \mathrm{II}$ activities (mother: $71 \%$, father: $69 \%$ ). In contrast, immunological determination of F II by ELISA showed normal concentrations in all family members (prop: $101 \%$, mother: $115 \%$, father: $93 \%$ ). The ability of FXa to cleave prothrombin was investigated by recalcification of the plasma samples and subsequent determination of prothrombin fragment $1+2$ by ELISA. A plasma from a 1 year old girl and a normal plasma pool served as controls. F1+2 concentrations generated in the plama of the family member were about 30\% lower than those of controls (prop.: 383, baby control: 590; mother: 800, father: 670 , adult control: $1040 \mathrm{nmol} / \mathrm{l})$. The fibrinolytic system was investigated by incubation of recalcified plasma samples for 2 hours at $37^{\circ} \mathrm{C}$ and subsequent determination of D-dimer and plasmin-antiplasmincomplexes (PAP) in the supernatant. Surprisingly, concentrations of crosslinked fibrin(ogen) products were 2 to 15 times higher than in controls (prop.: 153, baby control: 10; father: 70 , mother: 320 , adult control: 31 $\mu \mathrm{g} / \mathrm{l})$, while no differences were observed for PAP.

The data obtained so far characterize an abnormal prothrombin molecule with reduced cleavability by factor $\mathrm{Xa}$ and at best a residual activity for cleavage of fibrinogen and activation of FXIII.

Department of Transfusionmedicine and Hemostaseology, "Department of Pediatrics University of Marburg, +Behringwerke AG, Marburg, FRG. 
THERAPY OF SINUS CAVERNOSUS THROMBOSIS IN HEREDITARY PROTEIN C DEFICIENCY

A. Reckmann. A. Stürer. M. Gartenschläger. R. Besser*.H. Schinzel, LS. Weilemann 11. Med. and Neurologic Clinic, Johannes Gutenberg University Mainz, FRG

Objective: Thrombosis of sinus veins represents a rare complication of hereditary protein $\mathrm{C}$ deficiency. Usually full heparinization precedes a long term treatment with coumarin. Occasionally fibrinolysis has been advocated. As an alternative approach we tested low molecular weight heparins in patients with contraindications for thrombolysis.

Case report: A 42 year old patient suffered a car accident without visible cause. A scan displayed an epidural hematoma concerning the left frontal lobe. Headaches and minor word finding disorders regressed fast.

Twelve days after the accident a sensoric aphasia, a right-sided hemiparesis and focal seizures in the right arm could be observed. The cct scan did not show any differences to the initial examination i.e. the contusion and the rest of the hematom could be noted. Only the MRI-scan could secure the diagnosis of sinus vein thrombosis.

Low molecular weight heparin was continuously administered intravenously as well as phenytoin after an initial bolus. Thus, seizures and aphasia regressed and on the second day after initiation of this therapy the patient was asymptomatic.

Global tests of coagulation, the level of thrombin-antithrombin-lil-complex (TAT) and D-Dimer were continuously monitored. The dose of low molecular weight heparin was steered according to the levels of anti- $\mathrm{Xa}$ and TAT

With the elevation of TAT and D-Dimer paresthetic sensations in the right arm occured and regressed within minutes after augmentation of the dose of low molecular weight heparin. Two weeks after admission a second MRl-scan showed an incomplete recanalization of the thrombosed sinuses. The patient was dismissed on oral phenytoin and subcutaneous low molecular weight heparins (initially three times 5000 lU per day)

Conclusion: Low molecular weight heparin (Fragminim) seem suited for the therapy of acute venous thrombosis of cerebral sinuses. The molecular markers TAT and especially D-Dimer have proven to represent sensitive parameters for the detection of increased coagutation respective reactive fibrinolytic activity in patients with protein $C$ deficiency.
FALCIPARUM MALARIA: TISSUE FACTOR IN MONONUCLEAR CELLS Hemmer ChJ, Luther Th, Kotzsch M, Albrecht S, Caselitz J, van Lunzen J, Dietrich M.

In human faiciparum malaria, elevated Tissue Factor (TF) plasma levels correlate with disease severity and with the capacity of patient serum to induce TF in cultured vascular endothelial cells. Since TF expression is inducible in monocytes/macrophages and in the vascular endothelium (which are both involved in malaria), we asked whether these systems could contribute to circulating TF. To assess the role of the vascular endothelium, tissue sections (brain, kidney, lung, liver) from two patients who had died from severe falciparum malaria were stained with a monoclonal anti-TF antibody. Their vascular endothelium did not display stronger staining for TF than the endothelium of control patients who had died from unrelated diseases (septicemia was specifically excluded). Mononuclear Leukocytes (MNL) in the extravascular tissue, however, stained TF-positive at a somewhat higher rate in malarious patients than in controls. Almost no intravascular leukocytes were seen in malaria, while the capillary lumen was packed with falciparum-parasitized erythrocytes.

Next, we isolated MNL from the blood of a malarious patient (who later died from multiorgan failure) and a healthy control person by Ficoll-paque gradient centrifuga tion. The cells were disrupted by freeze-thawing and tested for TF antigen by sen sitive ELISA. Calculated per $10^{6}$ CD14 positive MNL's (i.e. presumably monocytes), $140 \mathrm{pg}$ TF $/ 10^{6}$ cells were found in malaria, and $60 \mathrm{pg} / 10^{6}$ cells in the control experiment. When total MNL's were compared instead, this difference was less obvious (11 vs $8 \mathrm{pg}$ TF $/ 10^{6}$ cells).

These preliminary data suggest that circulating monocytes (and possibly macrophages) contribute to TF in the plasma of patients with falciparum malaria. In addition to further experiments with monocytes. TF synthesis in the vascular endothelium will have to be analyzed by in-situ hybridization, since TF induction in both systems may be relevant in fatal complications of falciparum malaria.

Bernhard-Nocht-Institute of Tropical Medicine, Dept. of Medicine, 20359 Hamburg, Germany
Fatal complications by replacement of Fibrinogen in a patient with Afibrinogenemia. A case report

\section{F.Rommel, A.Trauner, M.Spannagl, R.Gärtner, W.Schramm}

A woman (31y) with afibrinogenemia and a history of moderate bleeding complications since childhood was substituted with fibrinogen during an amputation of necrotic DII/III, right foot. After arterial punction by central venous cannulation patient developed subcutaneous and mediastinal excessive bleeding. High fibrinogen replacement was started and bleedin stopped, but the patient developed deep thrombosis of the iliac, femoral and popliteal vein, a phlegmasia coerulea dolens of the left leg.

Labratory findings: No clottable protein was detected in pretreatmen plasma, no reactive epitope was found using different anti-fibrinogen antibodies. A normal coagulation system could be demonstrated after addition of fibrinogen or chromogenic thrombin substrates in vitro to the patients plasma. Under substitution of fibrinogen routine clotting tests become reactive

Collagen as well as ADP-induced thrombocyte-aggregation failed in pretreatment PRP. After fibrinogen replacement thrombocyte aggregation was normalized.

Clinical outcome: After onset of thrombosis of the left leg, fibrinogen substitution was modified, dose was reduced and bolus application changed to continous infusion. Endogenous fibinolysis reopened the venous system of the patient, but DI and parts of the skin of the left foot became necrotic.

Conclusion: The reported case suggest the close realtionship between fibrinogen substitution and thrombembolic complication in this patient after high dose $(>3 \mathrm{~g} / \mathrm{d})$ bolus substitution. A continous low dose $(1-2 \mathrm{~g} / \mathrm{d})$ replacement of fibrinogen with borderline reactivity in clotting tests should be prefered for long term fibrinogen replacement in patients with afibrinogenemia.

Haemostaseologische Abteilung, Medizinische Klinik, Klinikum Innenstadt der

Universität München, Ziemssenstraße 1, D-80336 München 


\section{VARIABLES THAT MIGHT EFFECT THE OUTCOME OF IMMUNE TOLERANCE THERAPY IN HAEMOPHILIACS WITH FACTOR VIII INHIBITORS}

S. Ehrenforth**, M. Funk*, G. Auerswald ${ }^{* * *}$ D. Mentzer*, I. Scharrer ${ }^{* \star}, W$. Kreuz $^{*}$

As yet no definite conclusion according variables that might effect outcome of immune tolerance (IT) therapy in haemophiliacs who have developed FVIII inhibitors could be drawn. Here we report our experience obtained over 14 years in the IT therapy of 21 children. Results: FVIII inhibitor elimination was achieved in 19/21 pts. in a median time of 4 months in high responders and 1.5 months in low responders. In HR pts. a tendency was observed between the initial FVIII dosage administered (U/ $/ \mathrm{kg} / \mathrm{d})$ and the median time needed to eliminate the FVII inhibitor: 3 months $(0.5-4.1$ months) with an initial FVIII dosage of $300 \mathrm{U}$ and 4.25 months $(2-14$ months) after an initial dosage of $200 \mathrm{U}$. Initial FVIII doses of $\leq 100 \mathrm{U}$ were associated with prolonged treatment time $(5,8,42$ months) or even with complete failure. In $2 / 4$ pts.with maximal FVIII inhibitor titres $>600 \mathrm{BU}$, no IT was achieved; in 1 pt. 42 months were needed. Conversely, there was no correlation when the maximal titre was $<600 \mathrm{BU}$. Favourable results of IT correlated relevantly with the FVIII exposure days between the initial FVIII inhibitor detection and initiation of IT therapy: $0.5-8.0$ months were needed in pts. with $\leq 14$ FVIII expo.days, 4.1-9.0 months in pts.with 15 to 21 expo.days. In $2 / 4$ pts. with $\geq 21$ expo.days, 14 and 42 months were needed to eliminate the inhibitor, in $2 / 4$ pts. no FVIII inhibitor elimination was obtained. No relevant correlation was observed when comparing the FVIII inhibitor titre or pts. age at the time when IT therapy was initiated and outcome. In 5 HR pts. in which the administration of high dose FVIII was discontinued, the outcome of IT therapy was deteriorated relevantly and associated with a prolonged treatment time in $3 / 5$ pts. sor even a failure (2/5).Conclusion: For a rapid FVIII inhibitor elimination it is important to start continuous administration of high dose FVIII early in the course of the disease or before repeated exposure to the antigen FVIII, in order to prevent rebooster effects, prolongation of elimination time and to save expense. Dept. of *Paediatrics and **Internal Med., Univ. Hospital, Frankfurt ***:Prof.-Hess-Kinderklinik, St. Jürgens Straße, Bremen

Platelet Counts and Recovery of factor Vill levels before, 15 and 30 MINUTES AFTER THE INFUSION OF DIFFERENT FACTOR VIII CONCENTRATES. H. Köstering, A. Coldewey, U. Söling, M. Unterhalt and J.U. Wieding

In acute bleeding complications in patients with hemophilia $A$ we could rely on the PTT as a single parameter to monitore the efficacy of factor VIII substitution. Since 1973, however, it was reported that the PTT test remained prolonged despite FVIII substituion. In one case, for example, the PIT was prolonged to $52 \mathrm{~s}$ after high dose administration of factor VIII resulting in a FVIII level of $110 \%$. Therefore we started a prospective study to investigate this phenomenon.

In patients with hemophilia $A(n=37)$ and von Willebrand Disease $(n=9)$ recoveries of FVIII, PTTs, platelet counts and other parameters were measured before, 15 and 30 minutes after administration of factor concentrates; all patients received 25 to $30 \mathrm{IE}$ per $\mathrm{kg}$ body weight.

After administration of concentrates from Immuno $(n=3)$, Intersero $(n=14)$ and Alpha $(n=4)$ the factor VIII recoveries corresponded well with PTT values. However, significantly prolonged PTTs were found in patients after $(n=5)$ treatments with Monoclate $(73,57$ and $58 \mathrm{~s}$ before, 15 and $30 \mathrm{~min}$ after infusion, respectively and after $(n=9)$ infusions of Haemate HS $(67,2 \mathrm{sec}, 52,4 \mathrm{sec}$ and $48,1 \mathrm{sec}$ before, $15 \mathrm{~min}$ and $30 \mathrm{~min}$ after infusion, respectively, with a factor VIII recovery of $87,8 \%$ in mean). Apart from PTT prolongation an increase of reptilase time was found in comparison with the above-mentioned three other FVIII concentrates. No significant differences between the groups were found in measuring factor XII and XIII, a2-antiplasmin, plasminogen, plasmin, fibrin monomer complexes and leucocytes.

Interestingly, in some patients treated with Heamate HS $(n=5)$ or Monoclate $(n=1)$ a drop of platelets by 20 to $35 \%$ was observed after infusion of both FVIII concentrates. In addition, the platelet function was disturbed (platelet aggregation induced by arachidonic acid). Four patients recovered from thrombocytopenia after switching to another FVIII concentrate; one patient additionally required Retrovir before an increase of platelet counts from 3000 to $61000 / \mathrm{ul}$ was observed. Further investigations are necessary to explain these results.

\author{
INACTIVATION OF HEPATITIS A VIRUS BY VAPOR HEAT \\ TREATMENT OF COAGULATION FACTOR CONCENTRATES \\ N. Barrett ${ }^{\star}$, H. Meyer, I. Wachtel, J. Eibl, F. Dorner
}

Cases of Hepatitis A virus (HAV) transmission by transfusion of whole blood, erythrocyte concentrates or plasma have been described. However until recently it was believed that transmission of HAV by purified human blood products did not occur. A number of recent publications, however, have described outbreaks of acute hepatitis $A$ in hemophiliacs who had received solvent / detergent treated $\mathrm{F}$ VIII concentrates. This treatment had been developed to inactivate enveloped viruses such as HIV-1 and would not be expected to inactivate non-enveloped viruses such as HAV. The vapor heat treatment procedures developed by Immuno were introduced in order to inactivate both enveloped and non-enveloped viruses and have been demonstrated in long term clinical trials to render products safe with respect to virus transmission. The efficacy of these procedures with respect to inactivation of HAV have now been evaluated in preclinical studies with coagulation factor concentrates. The vapor heat treatment used for Factor VIII concentrates Kryobulin ${ }^{\circledR}$ and Immunate ${ }^{\circledR}$ was demonstrated to inactivate at least 5.6 logs and 5.8 logs HAV respectively. Details of these studies and those with other coagulation factor concentrates will be presented.

*Immuno AG, Biomedical Research Center, A-2304 Orth a.d.Donau, Austria

327

\section{VIRUS INFECTION RISK IN CONGENITAL COAGULATION DEFECTS}

Margit Serban, Lucia Balasescu, Natalia Rosiu, Carmen Petrescu, Irina Iacob, Aurelia Bodea Blood and blood products are often rescuer therapies for noumerous haematological diseases. But we cannot ignore the major risk of transmitted infections. We investigated the peculiarities of the replacement therpy in haemopiliac patients traeted in Ist. Paediatric Clinic Timisoara (Romania).Absence of prophylactic therapy, the substitution treatment being restraint only for curativ indications;

-predominat using of nativ blood products provided by single or oligodonors and abcence of anti-viral vaccination

-use of blood products provided from apparently healhy donors -absence of HIV testing before 1990, HBs-AG testing since 1962. We tried to estimate the freqency and profile of transmitted infections during transfusionl therapy (hepatis A,B,C,D; HIV1/2; CMV) in nonvaccinated childrenwith haemophilia in dependence to the severity of the disease and the type of treatment and analyzed the long term conseqences. The spectrum of transmitted infections was quite different from those reported in other countries. Its most obvios features were:

-the absence of HIV infection, in spite of starting HIV tests 1990; -insignificant proportion of acute CMV infection $(4,2 \%)$;

-a high frequency of $\mathrm{HBV}$ infections $(77,14 \%)$

-high proportion of multiple hepatitis virus infection $(68,4 \%)$ -potential infectious risk in patients with IGM anti-HAV $(17 \%)$ The reasons of these particular circumstances are connected to: -The position of Romania in the range of medium/high endemicity areas for HAV and $\mathrm{HBV}$ and the absence of anti-HAV vaccination use of blood products provided by single or oligodonors; -presence of anti HBC positiv donors $(65 \%)$ with infections risk. The long term consequences of these infections might be at least considered serious, if we take into account that the proportion of $41,41 \%$ of haemopiliacs with chronic hepatitis or asymptomatic carrier status, is referred to the age of childhood; these association of $\mathrm{HBV}$ and $\mathrm{HCV}$ could have derogatory influence.

Clinica I-a Pediatria, 1900 Timisoara, Romania 
TISSUE FACTOR PATHWAY INHIBITOR AND HEMOHILIA

U.T. Seyfert, S. Perkins and E. Wenzel

A key event initiating blood coagulation after tissue injury is exposure of blood to tissue factor. Tissue factor pathway inhibitor (TFPI) is an effective inhibitor of factor VIIa/tissue factor mediated activation of factor $X$.

Patients and methods: In 20 hemophiliacs $(75$ determination points) TFPI values were determined using a dilute thromboplastin assay (according to Welsch DJ et al. :Thromb Res $64 ; 213-222,1991)$. An anti-TFPI antibody was kindly provided by 0. Nordfang, Novo, Gentofte/Denmark).

Results: Mean TFPI values in hemophiliacs did not differ from a normal population (TFPI $95.5 \%$ 42.5\%): After substitution therapy with factor VIII concentrates there was a decline of TFPI values (mean Delta $15.5 \%$, range $0-50 \%$ ). In 1 patient with an inhibitor there was a $50 \%$ decrease of TFPI values after FEIBA - substitution therapy. Depressing TFPI function diminishes bleeding and correlates with good hemostasis.

Conclusions: Some evidence has been provided to indicate that TFPI can inhibit factor VIIa/tissue factor complexes. Substitution therapy with concentrates indicates that TFPI functions as an inhibitor to feedback control of blood coagulation initiated by tissue factor. Further studies are necessary to clarify the biological role of the 3 known pools (endothelium, plasma, platelets) of TFPI.

Address: Klin. Haemostaseologie, Haus 75 , Unikliniken, D-66421 Homburg
PROTHROMBIN CONTAMINATING LP(a) LYS SUBFRACTION ENHANCES PROCOAGULATORY ACTIVITY (PCA) OF HUMAN MONOCYTES/MACROPHAGES P.Schuff-Werner1), J.Bredehöft 1), M. Helmhold1), H.Köstering ${ }^{2)}$ and V.W.Armstrong 1)

Lp (a) subfractions can be differentiated by their binding affinity to lysine (Lys ${ }^{+}$and Lys ${ }^{-}$). In cultured human monocytes Lp(a) Lys $^{-}$ induces surface expression of procoagulatory activity in a dose- and time-dependent manner, which cannot be explained by contamination with endotoxin. As shown by its high factor II activity the Lp(a) Lys $^{-}$subfraction seems to have proteolytic properties, which could be separated from Lp(a) by additional ultracentrifugation and which was identified as prothrombin using the thrombin-antithrombin test (TAT). The remaining prothrombin-free Lo(a) Lys still induced a 3 -fold stimulation of the basal PCA, whereas the Lp(a) Lys subfraction had no stimulatory effect. Purified prothrombin in physiological concentrations elicits an up to 100 fold stimulation of PCA in human monocytes/ macrophages. Prothrombin seems to be at least an equaliy good stimulator of PCA as endotoxin. The physiological importance of the descibed phenomenon remains to be clarified.

Department of Internal Medicine, Div. Clinical Chemistry 1) and Div. of Haematology/onkology ${ }^{2}$ University Clinics, Georg-August-University, Robert-Koch-Str. 40, D-37075 Goettingen, Germany
SYNTHESIS OF TISSUE FACTOR PATHWAY INHIBITOR (TFPI) IN HUMAN SYNOVIAL CELLS MAY CONTRIBUTE TO THE PREDILECTION OF JOINTS AS A BLEEDING SITE IN HAEMOPHILIACS

T. Brinkmann, H.Kähnert, W. Prohaska, O. Nordfang" and K. Kleesiek

The expression of tissue factor pathway inhibitor (TFPI) is described for endothelial cells and megacaryocytes. A synthesis of TFPI in hepatocytes was not found. We investigated the expression of TFPI in cloned human synovial cells and in human chondrocytes. Our results showed that these cells synthesise TFPI specific DNA transcription products. After isolating the total amount of mRNA from cultivated human chondrocytes and cloned human synovial cells a full-length TFPI CDNA was synthesised by reverse transcription and polymerase chain reaction. We used primers which hybridised in the noncoding region of the TFPI CDNA and generated an amplification product of about $1000 \mathrm{bp}$. Single endonuclease restriction sites in the non-complementary 5 -end of the primers were used for subcloning of the amplified DNA into the vector pUC 18. The TFPI specific DNA was confirmed by double stranded DNA sequencing.

The inhibitory activity of TFPI was determined by a chromogenic substrate assay. In the medium of human chondrocytes and cloned human synovial cells the inhibitory activity was $630-720 \mathrm{mU} / 10^{8}$ cells and 1080-1665 $\mathrm{mU} / 10^{\circ}$ cells, respectively.

The synthesis of tissue factor pathway inhibitor in human synovial cells and human chondrocytes seems to be an important regulator in the synovial system to obtain the balance between fibrinolytic and clotting reactions and to avoid inadequat fibrin deposition on the cartilage surface in the joints. In haemophiliacs factor $\mathrm{Xa}_{\mathrm{a}}$ generation exclusively depends on the factor VIla/tissue factor pathway. Thus, an inhibition of this pathway by TFPI results in a decreased coagulation in these patients and leads to massive bleeding into the joints even when only small vessels are injured.

Institut für Laboratoriums- und Transfusionsmedizin, Herz- und Diabeteszentrum Nordrhein-Westfalen, Universitätsklinik der Ruhr-Universität Bochum, Georgstraße 11, 32545 Bad Oeynhausen, Germany * Biopharmaceuticals Research, Novo Nordisk AVS, Niels Steensens Vej 1, 2820 Gentofte, Denmark

\section{PREVALENCE OF HEPATITIS AND HIV IN A GROUP OF HAEMOPHILIACS} H. Pollmann, H. Richter

This year several publcations discussed the safety of the factor concentrates in our times. So we analysed the data of our patients with severe haemophilia A and B for hepatitis $A$, hepatitis $B$, hepatitis $C$ and HIV who were undergoing substitution therapy at our center.

The following results were observed within the whole group: In $14(16 \%)$ out of 89 patients we found positive IgG for hepatitis A while $44(46 \%)$ out of 95 patients were to be found positive for hepatitis $C$. Out of 105 patients 16 $(15 \%)$ were found to HIV antibody positive.

Previously untreated haemophilic children under the age of 15 years (i.e. born after 1977) were exclusively treated with a pasteurized factor concentrate showed the following markers of viral infection:

Out of 35 patients none were found to be positive for hepatitis A. All 36 tested patients were negative for hepatitis $C$. Two other patients positive for hepatitis $C$ were transfused with other blood products in case of surgery and a third patient received a non-virusinactivated therapy for some weeks. No children younger than the age of 15 were found to be infected with HIV in our group.

In a group of 12 patients from Eastern Europe previously treated with cryoprecipitate we found positive hepatitis A markers in 5 out of 12 haemophiliacs. Hepatitis B serology was positive in 9 patients, 8 patients showed positive hepatitis $C$ markers. All 12 patients were to be found negativ for HIV infection. 
Replacement Therapy

332

CRITERIA TO CONTROL THE EFFICACY OF SUBSTITUTION THERAPY IN HAEMOPHLIACS - Fourth Annual Report

H. Pollmann, S. Linnenbecker and H.Jürgens

In haemophilia therapy we know the following criteria to verify the efficacy of substitution therapy of our days:

\section{Pettersson Score \\ Orthopedic outcome \\ Social integration}

Nevertheless all these parameters are founded in the frequency of previous bleedings into one and the same joint of haemophilic patients. So all these parameters show only the historical failure of our therapeutical efforts because all of them are not repairable for our patients. Following the results of the "North-West German Study Group of Haemophitia Treatment" we continued to control the frequency of joint bleedings in a prospective study from 1988 to 1993 in all patients treated in the Haemophilia Center of our Children's Hospital. To control the therapy by the frequency of bleadings we developed a computer assisted program called "HAEMOPAT"

Since we started prophylactic treatment with 20 to $25 \mathrm{IU}$ per kg BW every other day or three times a week after the 6th bleeding into the same joint within the period of 12 mounth ("the rule of six") we found no damage to that joint within the study period regarding the PETTERSSON-Score or ORTHOPEDIC OUTCOME. In addition the SOCIAL INTEGRATION measured by hospitalization, absent from school or work is normal in our patients.

During 1988 and 1990 prophylactic treatment was able to reduce bleeding episodes in case of more than 5 joint bleedings from 9.7 to 4.9 joint bleedings within the first year of changing therapy to prophylactic treatment in case of more than 5 joint bleedings.

University Children's Hospital Münster, Albert-Schweitzer-Str. 33, 48129 Münster
334

Clinical Efficacy of a New Factor IX Concentrate, IMMUNINE E. Berntorp*, K. Anderle, E. Eyster, M. Kunschak, G. Rivard

A new high-purity, vapor heated factor IX concentrate, IMMUNINE, was developed by IMMUNO A.G. in early 1991. After determination of its in vivo recovery and half-life ${ }^{4}$, an international study to investigate its clinical efficacy and safety in terms of adverse reactions was initiated. This study is ongoing. Entry criteria include factor IX deficiency, and acute bleeding or the need for surgical prophylaxis. For treatment of acute bleeding, endpoints are the time and number of infusions required to achieve control of bleeding (where the protocol gives dichotomous criteria for the definition of control of bleeding depending on the type of episode). For surgical prophylaxis endpoints are profuse intraoperative tissue-bleeding and post-operative bleeding complications. As of November 30, 1993, 43 male patients have been entered at clinics in Austria, Canada, Italy, and Switzerland. Age ranged from one to 69 years. Twenty-eight patients received treatment for a total of 85 bleeding episodes, four of these patients and 15 others required surgical prophylaxis in a total of 25 instances. The bleeding episodes included joint, muscle, soft tissue, mucous membrane and CNS hemorrhages. Surgical interventions included dental extraction, and orthopedic and open-heart surgery. The 85 bleeding episodes required between one and 11 infusions to be controlled, with a mean of 1.8 (SD 1.8) infusions. In all but one case, the time to control the bleeding was reported to have been within a satisfactory range; one severe ankle bleeding responded very slowly. In all cases of surgical prophylaxis neither profuse intraoperative bleeding nor postoperative bleeding complications occurred. Two mild adverse events (itching, tingling in the throat) and one moderate event (rash) were reported; the symptoms abated without intervention.

'M.C. Poon et al.. Clinical Study of Recovery and Half-Life of Factor IX Concentrate (Human) Vapor Heated, IMMUNO, IMMUNINE. Poster presented at the 20th International WFH Congress, Oct 12-17, 1992, Athens, Greece.

*Department for Coagulation Disorders, University of Lund, Malmö Genera! Hospita!, S-214 01 Malmö, Sweden

\section{COAGULATION STUDIES IN A PATIENT WITH HEMOPHILIA A AND ORTHOTOPIC LIVER TRANSPLANTATION}

Th. Eller, R.S. Roß, L. Volbracht, H. Herfahrt, J. Erhard, R. Scherer, F.W. Eigler, D. Paar

A 39-year-old patient suffering from severe hemophilia A (factor VIII:C $1.0 \%$ ) and liver cirrhosis secondary to a chronic aggressive hepatitis $\mathrm{C}$ underwent orthotopic liver transplantation for treatment of both cirrhosis and congenital coagulopathy. Before transplantation, we found a deficiency of the hepatic coagulation factors, a low platelet count, a high von Willebrand factor (vWf) concentration, and a high ristocetin cofactor activity in plasma. In platelets no $v W f$ was detectable. The analysis of plasma vWf multimers showed a supranormal structure.

The patient received 13.000 units factor VIII, 1.000 units ATIII, 3 fresh frozen plasmas, and 3 red cell concentrates in total before, during and after surgery to correct his coagulation defect. By increasing liver function two days after transplantation further substitution therapy was not necessary.

In postoperative controls we found normal factor VIII:C activities and normal vWf concentrations in plasma. The plasma multimeric structure of vWf normalized and the platelet vWf concentration began to increase to normal values. Simultaneously hepatic coagulation defect disappeared.

In our patient liver transplantation leads to a correction of the factor VIII:C deficiency, the abnormalities in the F VIII/ WWf complex as well as the hepatic coagulation defect.

Division of Clinical Chemistry and Laboratory Diagnostics, Department of Internal Medicine, University of Essen, Hufelandstr. 55, 45122 Essen, Germany

\section{FACTOR VIII:C "INHIBITORS" WITH AND WITHOUT INHIBITORY ACTIVITY}

W.Mondorf, J.Ehret, S.Zedlitz, J.Last, *E.Lenz, *W.Kreuz, I.Scharrer

Department of Internal Medicine and *Department of Pediatrics Universitiy Hospital, Frankfurt/M., Germany

In accordance with others we have found antibodies to factor VIII:C with and without inhibitory activity in plasma of hemophilia A patients. The specific inhibitory activity was defined by comparing the inhibitory activity determined by the Bethesda assay with quantitative results of an enzyme linked immunosorbent assay. Plasma samples from 50 adult and 18 infant hemophiliacs with and without Factor VIII:C inhibitors were assayed by both methods. Plasma samples of hemophliacs undergoing inhibitor treatment were assayed on various days during treatment. Differing from our previous reports we modified the ELISA protocol by using a higher plasma concentration and parallel background (human albumin) determination to achieve a higher sensitivity. The specific inhibitory activity was characteristic for individual inhibitor patients and did not change significantly during inhibitor treatment. All plasma samples containing an inhibitory activity of more than $1 \mathrm{BU}$ showed high ELISA results indicating that all investigated inhibitors were IgG immunoglobulines. Plasma samples with an inhibitory activity between 0 and 1 BU showed low but also markedly elevated ELISA results suggesting the presence of non-inhibitory factor VIII:C antibodies in some of the plasma samples. Antibodies to background (human albumin) were seen only among infant hemophilia A patients. 
LOCAL THERAPY OF CHRONIC VENOUS ULCERATION WITH FACTOR XIII: FIRST CLINICAL ASSESSMENT. G.Wozniak, H.Montag, J.Alemany

The treatment of chronic venous ulceration is even nowadays not sufficient in all patients. Due to our experience with factor XIII concerning a delayed wound healing in cases of deficiency and because of several reports about a dependency of chronic venous ulceration and $F \mathrm{XIII}$ deficiency, 16 potients with postthrombotic ulcerations have been treated locally with $F \mathrm{XIII}$.

The mean time period of uiceration was 3,2 years and all the patients (12 women, 4 men averaging 59,8 years) were resistant to our usually performed therapy during several times of stationary treatment in the past.

Within an averaging period of 3,3 weeks we found a distinctly improved tendency of granulation. At this time we can not make an objective judgement about an accelerated epithelization, but the granulation tissue was definetively tight and showed a distinctly decreased tendency of bleeding and secretion in combination with an easing on pain to the patient during bandaging.

In three potients we could prevent a graft failure after dermatoplasty due to the reduction of wound secretion after four days local theropy with $F$ XIII.

Local therapy with $F \mathrm{XIII}$ is a notable addition in our armamentarium to treat patients with postthrombotic ulcerations.

Dept. of Voscular Surgery, Knappschafts-Krankenhaus, Osterfelderstrasse 157, 46242 Bottrop
BIOCHEWTCAL CHARACTERTZATTON OF A DOIBLE VTRUS TMACTIVATED F VIII CONCENTRATE (OCPAVI SDPLUS): vWF PROPERTIES AND STABILTTY OF F VIII:C IN SOLUTION

A. Siegemund ${ }^{1}$, U. Budde ${ }^{2}$, S. Robinson 3

Recently F VII concentrates double inactivated by heat and chemical treatment were approved for treatment of patients with hemophilia A. To evaluate whether these treatments have detrimental effects on the stability of F VIII:C and/or on the structure of the vWF protein, we tested five lots of Octavi SDPIus. For each lot we determined the stability of F VIII:C in solution, $\mathrm{VWF}: \mathrm{Ag}$, ristocetin cofactor activity and the F VIII binding capacity of the VWF

This concentrates contained less VWF than F VIII $(65-85 \%)$ The F VIII binding capacity was normal in all lots. The smal 1 and intermediate multimers were present.

Thus the F VIII/YWF complex in Octavi SDPlus showed no obvious alterations compared to the SD inactivated product.

The clotting activity, the VWF:Ag and the ristocetin cofactor activity were found to be stable over a period of three days or more at different storage temperatures $\left(4^{\circ} \mathrm{C}, 20^{\circ} \mathrm{C}\right.$, $\left.37^{\circ} \mathrm{C}\right)$ either in the original container or in the cassette of a portable pump for contimuous infusion.

1 Centre of Internal Medicine University of Leipzig

2 Blood Transfusion Service AK Harburg, Hamburg

3 Detapharma, Langenfeld, Germany

\section{7}

EFFECTS OF C1 ESTERASE INHIBITOR (C1 INH) CONCENTRATE ON COAGULATION AND COMPLEMENT ACTIVATION IN-VITRO W. Nürnberger, I. Michelmann, S. Eckhoff-Donovan and U. Göbel

$\mathrm{C1} \mathrm{NH}$ is the major physiological inhibitor of the activated hageman factor and the classical pathway of complement (CP). C1 INH has been given intravenously at high doses (60 to 180 units per $\mathrm{kg}$ body weight) for treatment of capillary leakage syndrome (CLS) and/or septic shock.

We analyzed the influence of $\mathrm{Ct} \mathrm{INH}(0.1,0.3,1.0,3.0,10$ and 30 units per $\mathrm{ml}$ plasma) on the PTT (chromogenic substrate assay) and on antibody-induced complement activation (hemolytic activity, CH50). PTT was prolonged at addition of more than 1 unit/ml $\mathrm{C} 1 \mathrm{INH}(\mathrm{p}<0.02$, t-test), and reached 1.5- to 2-fold base-line values at 3 to 10 units $/ \mathrm{ml} \mathrm{C1} I \mathrm{NH}$. $\mathrm{CH} 50$ was moderate reduced compared to base-line values at addition of 10 to 30 units/ml $\mathrm{C} 1 \mathrm{INH}$ ( $p<0.02$, t-test), but strongly reduced values (lower than the control range) were only seen in 3 out of 8 samples under 30 units $/ \mathrm{ml} \mathrm{C1} \mathbf{I N H}$.

In the next step, the activation of CP was induced by incubation of plasma with $0.2 \mathrm{mg} / \mathrm{ml}$ plasmin over 20 hours at $37{ }^{\circ} \mathrm{C}$. CP activation was assessed by determination of the activation product $\mathrm{C} 4 \mathrm{~d}$. The starting value of $\mathrm{C} 4 \mathrm{~d}$ was $2.15 \mu \mathrm{g} / \mathrm{ml}$. C4d increased after 5 hours with (without) plasmin to $3.32(2.64) \mu \mathrm{g} / \mathrm{ml}$, and after 20 hours with (without) plasmin to 7.83 (6.45) $\mu \mathrm{g} / \mathrm{ml}$. When the plasma was coincubated with plasmin 10.2 $\mathrm{mg} / \mathrm{mi})$ and $\mathrm{CI} \mathrm{INH}(0.3$ units $/ \mathrm{ml}$ plasma), C4d was after 5 hours with (without) plasmin at $2.15(2.01) \mu \mathrm{g} / \mathrm{ml}$, and after 20 hours at 5.77 (6.16) $\mu \mathrm{g} / \mathrm{ml}$.

in summary, $\mathrm{Ct} 1 \mathrm{NH}$ in these experimental settings resulted (in concentrations similar to that used for sepsis or CLS) in prolongation of $P T$ and inhibition of plasmin induced activation of CP. Antibody induced activation of CP was only partially inhibited and at higher doses. We conclude, that the PTT should be monitored in patients receiving more than 60 units/kg $\mathrm{C}+\mathrm{INH}$.

Heinrich-Heine-University, Department of Pediatric Hematology and Oncology, Moorenstr. 5, D-40225 Düsseldort

Supported by the "Elterninitiative Kinderkrebsklinik e.V., Düsseldorf

\section{9}

DOUBLE VIRUS INACTIVATION OF FACTOR VIII CONCENTRATE (Octavi SDPlus) BY A COMBINED SD TREATMENT AND MODIFIED PASTEURIZATION $\left(63^{\circ} \mathrm{C}\right)$

L. Biesert, S. Lemon*, H. Suhartono, L. Wang, H. Rübsamen-Waigmann

The inactivation of both transfusion-relevant viruses and a large number of model viruses by a heat step introduced into the manufacture of Octavi, a very high purity FVIII concentrate, has been studied following established guidelines from the EU CPMP Ad Hoc Working Party on Biotechnology/Pharmacy. In-process samples of Octavi obtained after the first virus inactivation step with solvent/detergent were stabilised, heated to $63^{\circ} \mathrm{C}$ in solution and then spiked with vinus-containing cell culture supernatant. Samples taken at different times in order to construct inactivation curves were titred on susceptible cells. Infectivity was determined by endpoint dilution. The virus titres were calculated according to Spearman-Kerber. Further studies with the flavivirus BVDV, bovine parvovirus and reovirus type 3 are in progress.

\begin{tabular}{|c|c|c|}
\hline Virus & $\begin{array}{l}\text { Reduction factor } \\
\log _{10} \text { TCID } 50\end{array}$ & $\begin{array}{c}\text { Time necessary for } \\
\text { complete inactivation }\end{array}$ \\
\hline $\begin{array}{l}\text { HIV-1 } \\
\text { HSV-1 } \\
\text { PRV } \\
\text { HAV } \\
\text { Polio-1. } \\
\text { Cox-B6 }\end{array}$ & $\begin{aligned} \geq 9.0 & (n=2) \\
\geq 5.7 & (n=2) \\
\geq 5.3 & (n=1) \\
\geq 5.6 & (n=1) \\
\geq 7.5 & (n=2) \\
4.7 & (n=1)\end{aligned}$ & $\begin{array}{l}\leq 240 \mathrm{~min} \\
\leq 240 \mathrm{~min} \\
\leq 120 \mathrm{~min} \\
\leq 480 \mathrm{~min} \\
\leq 480 \mathrm{~min} \\
600 \mathrm{~min}\end{array}$ \\
\hline
\end{tabular}

The combination of SD treatment and a heating step performed under more stringent conditions than conventional pasteurization leads to a factor VIII preparation with a higher safety standard than monoinactivated products.

Georg-Speyer-Haus, Paul-Ehrlich-Str. 42-44, D-60552 Frankfurt, FRG * The University of North Carolina at Chapel Hill, USA 
340

VIRUSSAFETY OF FEIBA ${ }^{\circledR}$ S-TIM4:

INACTIVATION AND PARTITIONING OF HIV-1 DURING MANUFACTURE N. Barrett, G. Pölsler, P.L. Turecek, F. Dorner*

Efficient virus inactivation procedures are applied in the manufacture of plasma derivatives from pooled human plasma, which in addition has been tested for contaminating viruses. Depending on the fractionation processes and fractionation steps used, partitioning alone may be sufficient to render certain plasma products safe. Thus, in the early $1980 \mathrm{~s}$, aithough HIV-1 was transmitted on a large scale through factor VIII concentrates, millions of doses of immunglobulin manufactured from the same plasma pools never transmitted HIV-11. Also for the Antiinhibitor Coagulant Complex, FEIBA ${ }^{2}$, it was postulated, that this product never transmitted HIV even without any specific virus inactivation step ${ }^{2}$.

In this study the capacity of various production steps of FEIBA ${ }^{\circledR}$ to remove and/or inactivate HIV-1 was investigated. Samples of intermediate products from the manufacturing facility were spiked with virus. The respective production step was carried out at a laboratory scale, the titer of HIV-1 was determined before and after each step. To prove the comparability of production and laboratory scale the content of FEIB-activity was determined in both the sample from the production and the laboratory. The reduction factors of HIV-1 for the whole fractionation process will be discussed according to EC/CPMP guidelines ${ }^{3}$.

As a specific virus inactivation step FEIBA ${ }^{2}$ is subjected to vapour heating. The intermediate lyophilisate of the plasma fraction is moistened homogeneously and then treated under inert gas and increased pressure at $60^{\circ} \mathrm{C}$ for 10 hours and $80^{\circ} \mathrm{C}$ for 1 hour. Vapour heating does not require protein stabilisation. Both steps of this virus inactivation were spiked with virus and a reduction factor of $\geq 11$ for HIV- 1 could be determined.

The study demonstrates an overall reduction factor of $>6$ only by virus partitioning throughout the manufacturing process.

1 Wells, M.A., et al, Inactivation and Partition of Human T-Cell Lymphothrophic Virus, Type III, During Ethanol Fractionation, Transfision, 26:210-213 (1986)

2 Negrier, $\mathrm{C}$., et $\mathrm{Al}$., Multicentric Retrospective Study on the Utilization of FEIBA ${ }^{*}$ in France in Patients with Factor Vill and Factor IX Inhibitors, 2. Int. Symp. on Inhibitors to Coagulation Factors, Chapel Hill (1993) 3 Commission of the European Communities (1991), Ad Hoc Working Party on Biotechnology/Ph

Note for Guidance, Validation of Virus Removal and Inactivation Procedures, Ili/81 15/89-EN

* Immuno AG, Industriestraße 67, A-1221 Wien, Österreich

A DOUBLE VIRUS-INACTTVATED, HIGHLY PURIFIED FACTOR VIICONCENTRATE

H. Schwizn, M. Stadler, DJ. Josic*, F. Bal, W. Gehringer, I. Nor and R. Schūtz

In an assessinent of the risks of virus transmission by clotting concentrates it is clear that the currently practised procedures for virus inactivation are not equally effective against all types of viruses; neither a pastearisation nor the Solvent Detergent (SDD) process alone are adequate enough to inactivate viruses that are strongly resistant to beat and organic solvents. In this connection, human parvo virus B19 and hepatitis A virus (HAV) are of particular concern. In order to improve this situation which still poses a risk to the haemophiliac patients, we have developed a more effective pasteurisation process that could be easily applied to our factor VIII (FVIII)-process in addition to the S/D-treatment already well-established. We were prompted to initiate experiments using temperatures above $60^{\circ} \mathrm{C}$ by two recent publications, which demonstrate that HAV becomes instable at temperatures exceding $62^{\circ} \mathrm{C}$. It is the prupose of this paper to present our progress in: - achieving a pasteurisation procedure for $F V I I I$ at $63^{\circ} \mathrm{C}$ for 10 hours with no discernible change in the structure of the factor VIII/von Willebrand factor (FVII/VWF)-complex owing to a newily developed composition of stabilizers

- applying this pasteurisation procedure to a purified FVII fraction, that has already been submitted to a S/D-treatment: Doing so, we are able to perform two independent vinas inactivation steps, as has been recommended (IABS-Meeting in Cannes, France, November 1992)

- introducing a second purification step on an anion exchange resin, achieving an additional virus reduction over our presently manufactured FVIII preparation

Numerous animal studies proved the double virus-inactivated FVII concentrate to be well-tolerated and without side-reactions. The effectivity of the $63^{\circ} \mathrm{C}$ heating step against different types of nor-enveloped and enveloped viruses is published elsewhere

Tresent address: Octapharma Pharmazeutika Produktionsges.m.b.H, Oberlaaer Straße 235, A-1100 Wien, Austria

EIN ZWEIFACH VIRUSINAKTTVIERTES, HOCHGEREINIGTISS FAKTOR VIIKONZENTRAT
342

EXTRACTION OF TRITON X 100 AND ITS ANALYSIS IN HUMAN PLASMA, VIRUS INACTTVATED BY THE SOL VENT / DETERGENT METHOD A. Strancar, H. Schwinn and DJ. Josic*

For the inactivation of lipid-enveloped viruses during the production of fresh frozen and lyophylized human plasma the solvent/detergent method developed by Horowitz at al. was applied (Horowitz, B.; Bonomo, R., Prince, A.M. Chin, S.N., Brotman, B. 8 Shulman, R.W. Blood 79 (1992), 826). In this process, the solvent tri-n-butylphosphate is removed by extraction with castor oil. The removal of non-ionic detergent Triton $X$ 100 is performed by solid-phase extraction using reversed-phase. Different polymer- and silica based supports were tested. The highest capacity for Triton X 100 was achieved with $\mathrm{C}_{18}$ silica gels. These supports bound more than $0.1 \mathrm{ml}$ Triton $X 100$ per one inl support. In practice, none of the proteins, e.g. clotting factors, bound to the support and therefore, flow through the column. Their biological activity was hardly affected. Another main application of such sugars is far quantitative: detergent analysis during the production process. The use of specisl colunns allowing direct sarmple injection could be introduced It is a simple method for very fast in-process analysis of Triton X 100 in human plasma by reversed-phase chromatography under isocratic conditons.

*Present address: Octapharma Pharmazeutika Produktionsges.m.b.H. Oberlager Straße 235, A-1 100 Wien, Austria

ENTFERNUNG VON TRTTON X-100 UND DESSEN BESTTMMUNG DM MENSCHLICHEN PLASMA, NACH VIRUSENAKIIVIERUNG MTT DER SOLVENTIDETERGENT METHODE

PURIFICATION OF FACTOR VIII AND VON WLLEBRAND FACTOR FROM HUMAN PLASMA BY ANION EXCHANGE CHROMATOGRAPHY DJ. Josic*, H. Schwinn, M. Stadler and A. Strancar

Factor VII (anti-hemophilia A factor) is isolated from human plasma, Purification is carried out by a combination of precipitation and chromatographic procedures. The precipitate after cryo-precipitation is dissolved in the buffer, and bulk proteins such as prothrombin factors, $\mathrm{FI}$ and $\mathrm{FV}$ are removed by adding of $\mathrm{Al}(\mathrm{OH})_{3}$ and subsequent cooling down to $16^{\circ} \mathrm{C}$. This aluminum hydroxide precipitation also removes several additional proteins from the clotting cascade. Although only traces of such proteins, e.g., factor $\mathrm{Xa}$ are found in the cryoprecipitate, they may activate factor VIII during subsequent steps of the isolation process, thereby reducing both yield and stability of the product. The first step in vinus inactivation is achicved through the effect of a non-ionic detergent such as Triton X 100 or Tween 80, and a solvent, e.g., Trin-n-butyl-phosphate (TnBP). By a stibsequent anion exchange chromatography step, a highly enricheof product is isolated, consisting of a complex formed by factor VII and von Willebrand factor (F VIII-vWF). This treatment also reduces the virus-inactivating reagents to quantitites in the low pptn range. The second step in virus inactivation is aimed specifically at the non-enveloped viruses and consists of pasteurization at tenuperatures greater than $60^{\circ} \mathrm{C}$ for 10 hotrs. In order to preserve the activity of the clotting factor VIIl during heating, sugars and amino acids are added as stabilizers. They are subsequently removed by ion exchange chromatography, along with possibly denthering proteins. In this step, additionsl vof is removed. Through the addition of stabilizers, between $80 \%$ and $90 \%$ of the initial activity of factor VIII is preserved during the modified pasteurisation. The twofold virus inactivation solvent-detergent treatment and pasteurisation allows the destruction of both liped enveloped and non-enveloped viruses. During this procedure factor VIII is stabilized through the high content of von Willebrand factor. The complex consisting of factor VII and von Willebrand factor can be dissociated by adding calcium ions. Subsequently both glycoproteins from this complex are separated from one another by further chromatography.

*Present address: Octapharma Pharmazeutika Produhtiousges.m.b.H., Oberiager Straße 235, A-1100 Wien, Austria

REINIGUNG VON FAKTOR VIII UND VON WLLEBRAND FAKTOR AUS MENSCHLICHEM PLASMA MIT ANIONENAUSTAUSCHER-CHROMATOGRAPHIE 
RECOVERY AND ELIMINATION HAIF-LIPE OF DOUBLY VIRUSINACTIVATED PROFITATE"

G. Müller Profilate
plasma and concentrated by chromatographic processes. plasma and concentrated by chromatographic processes. Recentiy the solvent-detergent-procedure for viral inthe severe-heat-treatment for 72 hours at $80^{\circ} \mathrm{C}$, in order to inactivate non-enveloped viruses. The aim of the present screening examination was to document initial clinical experiences with the new preparation. Five patients suffering from hemophilia $A$ were given 2.000 to 3,000 I.U. of Profilate" for 10 minutes intravenously. The preparation proved to be well tolerated and showed good solubility. Nelther during nor after the injection of the preparation did any adverse events occur. A physical examination at the end of the treatment did not show any changes to the initial examination findings. In the course of the adjustment of the patient to the preparation the current recovery and elimination halfpreparation the current recovery and elimination life were determined. The patients treated with Frofilate did show eatisfactory levels of factor VIII which suggests a good therapeutic potency of the prepara-
tion. When compared to predecessors and to other factor VIII-concentrates the average recovery and elimination half-1ife of Profilate did not show any basic deviations. For some patients also recovery and elimination half-iffe results of previous examinations were included. A comparison of those results to the new findings is also an indication that the insertion of the new virus inactivation step (severe heat treatment at $80^{\circ} \mathrm{C}$ for $72 \mathrm{~h}$ ) into the manufacturing process of Profilate" does not result in a relevant change of recovery ana elinination half 1 ife while unchanged.

Hospital for Internal Medicine

University of Halle

Magdeburger str. 2

06112 Halle-Wittenbers

\section{5}

DEVELOPMENT OF A DOUBLE VIRUS-INACTIVATED ANTIHAEMOPHILIC EACTOR CONCENTRATE IN A READY - FOR-USE-SYRINGE

P. Bhattacharya

In order to increase the viral safety of a factor VIII concentrate in a ready-for-use-syringe another step of virus-inactivation (Severe Heat Treatment, $80^{\circ} \mathrm{C} / 10 \mathrm{~h}$ ) was added to the

manufacturing process.

Thus the viral safety regarding enveloped and nonenveloped viruses was increased.

The product characteristics remained unchanged.

The manufacturing process of this double virus inactivated factor VIII concentrate (Profilate ${ }$, Alpha Therapeutic GmbH, Langen, Deutschland) runs as follows:

Profilate $e^{R}$ is manufactured from pooled human plasma by a Polyethylene Glycol (PEG) procedure, followed by the first virus-inactivation step: SD-Treatment (TNBP/Tween 80 ) at $27^{\circ} \mathrm{C}$ for $\mathrm{six}$ hours. The SD AHF solution is purified by an affinity chromatography process and sterile filtration. The sterile AHF solution is filled into clean sterilized containers, frozen, dried under vacuum and heated at $80^{\circ} \mathrm{C}$ for 72 hours (second virus-inactivation step). The double virus-inactivated bulk is reconstituted, sterile filtered, filled into the syringe and lyophilized again. Then the syringe manufacturing procedure is completed.

*Present address:Alpha Therapeutic Corporation, 5555 Valley Boulevard, Los Angeles, California 90032 , USA
ACUTE TOLERABILITY AND PHARMACOKINETICS OF DOUBLY VIRUSINACTIVATED PROFILATE' IN PATIENTS SUPFERING FROM VON WILLEBRAND-JÜRGENS - SYNDROME D. Franke, G. Lutze

Profilate"is a chromatographically purified, virally safe factor concentrate. The manufacturing process of this preparation has recently been supplemented by an additional heat inactivation step for 72 hours at $80^{\circ} \mathrm{c}$. In the present screening examination four patients suffering from known von-willebrand-Jürgens-syndrome were given 6,000 to 8,000 I. O. of additionally heat-treated Profilate" during 10 - 15 minutes intravenougly. The absence of adverse events, the constant measured values for blood pressure, pulse and body temperature and the examination results after the end of the treatment which did not show any pathological findings suggest a constantly rood tolerability of Profilate. Bleeding times could could be normalized in all four patlents and compared to the inltial values they could be reduced significantly. In those cases where the bleeding time had previously been prolonged the effect was considerably more marked than in cases where the bleeding time had been in the normal range. FVIII:C increased from $0.62 \mathrm{I} .0 . / \mathrm{ml}$ to 2.09 I. U. $/ \mathrm{ml}$, RCof increased from $0.26 \mathrm{I} .0 . / \mathrm{ml}$ to $1.26 \mathrm{I} . \mathrm{V} . / \mathrm{ml}$ and VWF: $\mathrm{Ag}$ from $0.62 \mathrm{I} .0 . / \mathrm{ml}$ to $2.6 \mathrm{I} . \mathrm{D} . / \mathrm{ml}$ after injection. The graphs for FVIII:C, RCof, VWF:Ag did not show any differences for any of the four pattents. These changes were sufficient to reach a balanced hemostagis. Especially the comparison to a previous examination indicates that the insertion of the new virus inactivation atep (severe heat treatment $80^{\circ} \mathrm{C}$ for $72 \mathrm{~h}$ ) into the manufacturing process of Profilate has not caused a relevant change in the tolerability or in the measurable effectivity in von willebrand-Jurgens-syndrome.

Head of Hemophilia Centre

Department of Internal Medicine University of Magdeburg 06112 Magdeburg

* Head of the Coagulation Laboratory of the Institute for Clinical Chemistry, Laboratory and Diagnostics Wniversity of

POSSIBLE ANAPHYLAXIS TO POLYETHYLENGLYKOL (PEG) IN A RECOMBINANT ANTIHEMOPHILIC FACTOR PREPARATION IN A FIVE-YEAR-OLD HEMOPHILIAC A Case report

Hulpke-Wette, M., Kamps, K., Gahr,M.,

We report on a five-year-old boy with severe hemophilia A (less than $1 \% \mathrm{~F}$ VIII activity) whose self treatment was changed from the application of an intermediate purity plasma derived. F VIII preparation to recombinant antihemophilic factor VIII (Recombinate ${ }^{R}$, Baxter).

Shortly after the fourth application of $250 \mathrm{U}$ of Recombinate ${ }^{\mathrm{R}}$ he developed a wheeze, dyspnea, temperature up to $39^{\circ} \mathrm{C}$ and a shivering fit. These symptoms lasted for two hours.

Two days later during the next application of $250 \mathrm{U}$ the application had to be stopped after half of the dosage because of severe dyspnea and a shivering fit. We suppose a hypersensitivty reaction to one of the stabilizing factors containing in this recombinant antihemophilic factor VIII preperation e.g. Polyethylenglykol (Macrogol 3350) or human albumin. Few reports on anaphylaxis to PEG are found in the literture (Kwee, Dolovish, 1982, J Allergy Clin.Immunol.

,69, 138)

We are planning to perform skin testing against PEG in order to confirm our suspicion of PEG being the factor responsible for this anaphylactic reaction. 